TE WHARE WĀNANGA O TE ÜPOKO O TE IKA A MĀUI

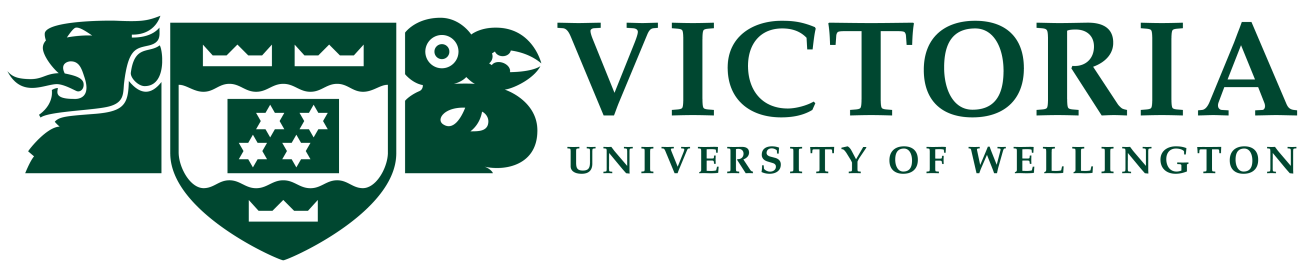

\title{
From the Superatom to a Large Variety of Supermaterials: \\ A Theoretical Study
}

\section{Dissertation}

in fulfillment of the requirements

for the degree of Doctor of Philosophy

by

Julia Schacht

School of Chemical and Physical Sciences

Victoria University of Wellington 

You see things; and you say 'Why?'

But I dream things that never were; and I say 'Why not?'

George Bernard Shaw 



\section{Abstract}

Metal clusters have been a subject of interdisciplinary research for many years as they act as a bridge between atoms and solid-state materials. In particular, clusters that show distinct thermodynamic stability and unusual atom like behavior, with an electronic shell structure that exhibits a superatomic nature, have attracted considerable attention. The concept of clusters behaving as individual atoms and furthermore mimicking the chemistry of specific elements directly leads to the idea of using those nanoparticles as building blocks for new functional materials. Furthermore, it is interesting that one can change the properties of cluster assembled materials by solely changing the properties of the individual clusters involved.

In this work, various factors affecting superatomic assemblies are identified and critically analyzed within the means of first-principles computations. The icosahedral gold cluster $\mathrm{Au}_{13}\left[\mathrm{RS}(\mathrm{AuSR})_{2}\right]_{6}$ has been chosen as a model system to study the tunability of the electronic structure using single atomic impurities. In this context the doped clusters were found to be tunable such, that they reveal atomic properties, e.g. electron affinities similar to individual halogen atoms. In addition, the choice of ligands protecting the clusters is evaluated regarding the stability of the whole cluster and the involvement of the ligands in creating the superatomic structure. The latter was found to be important when thinking of orbital overlap in superatomic assemblies.

In a next step the knowledge gained is used to investigate cluster-cluster interactions and detect pairs of clusters that are good candidates to create new superatomic materials. Furthermore basic principles regarding cluster assemblies are established and partially tested in an experimental collaboration studing the structure of an $\mathrm{Au}_{9}\left(\mathrm{PPh}_{3}\right)_{8}-\mathrm{C}_{60}$ assembly. 
Beyond the investigation of individual gold clusters and gold cluster materials, the electronic structure of binary solid state materials consisting of ligand protected transition metal-chalcogen clusters and fullerenes, as synthesized by Roy et al., is presented. This study shows an intermediate case of non-tunable clusters and furthermore displays the partial loss of the superatomic character of the transition metal chalcogen clusters due to charge transfer.

An experimental collaboration conducted in cooperation with the research group of Prof. Beate Paulus in Berlin proceeds even further and investigates the absorption of water on non-superatomic aluminumoxo fluoride clusters. 


\section{Acknowledgments}

This dissertation ends an important and beautiful, but also very stressful chapter of my life.

I would like to take the opportunity to express my gratitude to all the people that have supported me in so many different ways over the last three years, and have made this time so special to me.

First and foremost, I want to thank my supervisor assoc. Prof. Dr. Nicola Gaston who gave me the opportunity to come the long way to Wellington and be part of the scientific community in New Zealand. Nicola gave me the freedom to develop my own ideas, while at the same time guiding my scientific development. She was always on hand when I needed advice or wanted to discuss the big riddles my research constantly provided.

My special thanks goes to my secondary supervisor Dr. Matthias Lein, who welcomed me as part of his research group when Nicola got the opportunity to become associate Professor at Auckland University. His broad range of expertise and his curiosity have not only contributed to the progress of my thesis, but also broadened my general scientific horizon.

Moreover, I want to show my gratitude to Prof. Dr. Beate Paulus, who kept supporting my scientific development past my Master thesis and gave me the opportunity to join her research group a second time to be part of a very interesting project. 
Beyond that, I want to show my appreciation to the New Zealand eScience Infrastructure who provided my with computer resources and technical support.

There are many collaborators and colleagues I had the pleasure to work with during the past three years. I particularly want to emphasize Dr. Lukas Hammerschmidt who has been a great help with multiple ideas and suggestions. Furthermore, I am thankful for the the experimental collaborations conducted with the working group of Prof. Dr. Erhard Kemnitz and Dr. Jonathan Halpert. My big thanks goes to all the members of assoc. Prof. Gaston's, Dr. Lein's and Prof. Paulus's research groups for the great working atmosphere, all the laughter, friendship and for an amazing time.

I am so very grateful for all the lovely people who crossed my path in the past few years and made my time in New Zealand a big adventure.

Finally, I want to thank my whole family for all their love, support and understanding during a long period of study. 


\section{Contents}

1 Introduction 9

L. Metal Clusters . . . . . . . . . . . . . . . . . . . . . . . 9

1.2 Superatom Model . . . . . . . . . . . . . . . . . . . . . . . 10

1.3 Establishment and Application of the Superatom Concept . . . . . . . . 13

L.3.1 Bare Metal Clusters . . . . . . . . . . . . . . . . . . . . . 13

1.3 .2 Ligand Protected Metal Clusters . . . . . . . . . . . . . . . 15

1.3 .3 Superatomic Clusters as new Building Blocks . . . . . . . . . . . 19

1.4 Limits of the Superatom Model . . . . . . . . . . . . . . . . . . . 21

1.5 Non-Superatomic Custers . . . . . . . . . . . . . . . . . . . . . . . . 22

1.6 Summary . . . . . . . . . . . . . . . . . . . . . . 23

$\begin{array}{llr}2 & \text { Theory } & 26\end{array}$

2.1 The Schrödinger Equation . . . . . . . . . . . . . . . . . . . . . . . 26

2.2 Hartree-Fock $\ldots \ldots \ldots \ldots$

2.3 Density Functional Theory . . . . . . . . . . . . . . . . . . . . . . . . . . 32

2.3 .1 Kohn-Sham Equations . . . . . . . . . . . . . . . . . . . . . . 33

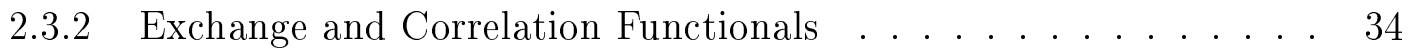

2.3 .3 Dispersion Corrections $\ldots \ldots$. . . . . . . . . . . . . . . 36

2.3 .4 Basis Sets and Effective Core Potentials . . . . . . . . . . . . . . 37

2.4 Quantum Theory of Solids . . . . . . . . . . . . . . . . . . . . . 40

2.4.1 Plane Wave Basis Set . . . . . . . . . . . . . . . . . . . . . . . . . 43

2.4 .2 Band Structure and Density of States . . . . . . . . . . . . . . . . 44

2.4 .3 Computational Details . . . . . . . . . . . . . . . . 46 
3 Tunability of Superatoms 47

3.1 Introduction . . . . . . . . . . . . . . . . . . 47

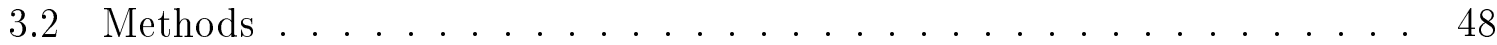

3.3 Discussion . . . . . . . . . . . . . . . . . . . . . . . . . . . . 48

3.4 Conclusion . . . . . . . . . . . . . . . . . 66

4 Transition Metal Cluster Assemblies 68

4.1 Introduction . . . . . . . . . . . . . . . . . . . . . . . . . . . . . . 68

4.2 Methods . . . . . . . . . . . . . . . . . . . . . . . . . . . . . . . . . . . . . . 69

4.3 Discussion . . . . . . . . . . . . . . . . . . . . . 70

4.3 .1 Electronic Structure . . . . . . . . . . . . . . . . . . 74

4.3 .2 Superatomic Structure . . . . . . . . . . . . . . . . . . . . . . 77

4.4 Conclusion . . . . . . . . . . . . . . . . . . . . . . . . . . 88

5 Screening of Potential Superatomic Compounds 91

5.1 Introduction . . . . . . . . . . . . . . . . . . . . . . 91

5.2 Methods . . . . . . . . . . . . . . . . . . . . . . . 9 92

5.3 Discussion . . . . . . . . . . . . . . . . . . . . . . 93

5.3.1 Superatomic Gold Clusters as Electron Donor Building Blocks . . 94

5.3.1.1 $\mathrm{Au}_{12} \mathrm{Al} \mid \mathrm{RS}(\mathrm{AuSR})_{2}{ }_{6}{ }_{6} \mathrm{C}_{60}$ Assembly . . . . . . . . . . 97

5.3.1.2 $\mathrm{Au}_{12} \mathrm{Al} \mid \mathrm{RS}(\mathrm{AuSR})_{2}{ }_{6}{ }_{6}-\mathrm{B}_{11} \mathrm{H}_{14}$ Assembly . . . . . . . . . . 100

5.3.2 Superatomic Gold Clusters as Electron Acceptor Building Blocks 104

5.3.3 Superatomic Gold Clusters as Electron Donor/Acceptor Building

Blocks . . . . . . . . . . . . . . . . 107

5.3 .4 Comparison . . . . . . . . . . . . . . . . 111

5.4 Conclusion . . . . . . . . . . . . . . . . . . . . . 115

6 Ligands Protecting the Clusters 118

6.1 Introduction . . . . . . . . . . . . . . . . . . . . . . 118

6.2 Methods . . . . . . . . . . . . . . . . . . . . . . . . . . 119

6.3 Discussion . . . . . . . . . . . . . . . . . . . . . . . . . . . 119

6.4 Conclusion . . . . . . . . . . . . . . . . . . . 125 
\begin{tabular}{llr|r}
7 & Experimental Collaborations & 127
\end{tabular}

$7.1 \quad$ First Steps Towards the Understanding of $\mathrm{Au}_{9}\left(\mathrm{PPh}_{3}\right)_{8}-\mathrm{C}_{60}$ Compounds . 127

7.1.1 Introduction . . . . . . . . . . . . . . . . . . 127

7.1.2 Methods . . . . . . . . . . . . . . . . . . . . . . . . . . 128

7.1.3 Discussion . . . . . . . . . . . . . . . . . . . . . . . . . . . . . 128

7.1.4 Conclusion . . . . . . . . . . . . . . . . . . . 134

7.2 Investigation of the Interaction Between Aluminum Oxide Fluoride Clus-

ters and Water . . . . . . . . . . . . . . . . . . 135

72.1 |ntroduction . . . . . . . . . . . . . . . . . 135

7.2 .2 Methods . . . . . . . . . . . . . . . . . . . . 136

723 Discussion . . . . . . . . . . . . . . . . . . 137

7.2 .3 .1 Conclusion . . . . . . . . . . . . 150

8 Summary 151

$\begin{array}{llr}9 & \text { Bibliography } & 154\end{array}$ 


\section{List of Figures}

1.1 Harmonic, Intermediate and Square-Well Potential . . . . . . . . . . . . 12

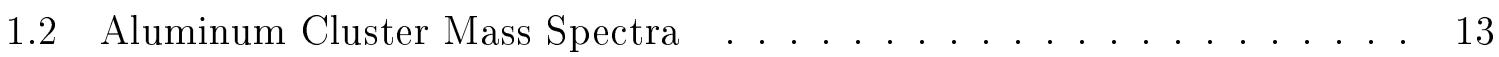

$1.3 \quad$ Electronic Shell Structure of a Chlorine Atom and $\mathrm{Al}_{13}^{-}$. . . . . . . . . . 14

$1.4 \mathrm{Au}_{102}(p-\mathrm{MBA})_{44}$ Cluster $\ldots \ldots \ldots \ldots \ldots$. . . . . . . . . . . 16

$1.5 \mathrm{Au}_{25}\left(\mathrm{SCH}_{2} \mathrm{CH}_{2} \mathrm{Ph}\right)_{18}^{-}$Cluster $\ldots \ldots \ldots \ldots \ldots \ldots$

1.6 TEM Pictures of Binary Cluster Assembled Materials . . . . . . . . . 20

1.7 Materials Consisting of Fullerenes and Transition Metal Containing Clusters 21

2.1 Wigner-Seitz Cell Construction Scheme . . . . . . . . . . . . . . . . . 43

$3.1 \mathrm{Au}_{13}\left(\mathrm{RS}(\mathrm{AuSR})_{2}\right)_{6}$ Cluster with Doping Sites $\ldots \ldots \ldots$. . . . . . . . . 49

3.2 PDOS of Differently Doped $\mathrm{Au}_{13}\left(\mathrm{RS}(\mathrm{AuSR})_{2}\right)_{6}$ Clusters $\ldots . . . . . \quad 51$

3.3 Superatomic $S$ Orbital of the Undoped, the Pt and Ge Doped $\mathrm{Au}_{13}$ Cluster 52

$3.4 \quad$ PDOS and PLDOS of the $\mathrm{Au}_{12} \mathrm{Pd}^{2-}$ Cluster . . . . . . . . . . . . . . . . 53

$3.5 \quad$ PLDOS of Differently Doped $\mathrm{Au}_{13}\left(\mathrm{RS}(\mathrm{AuSR})_{2}\right)_{6}$ Clusters . . . . . . . . . 54

3.6 PDOS of the Pt Doped $\mathrm{Au}_{13}$ Cluster with Different Charges . . . . . . . 55

3.7 Charge Density of the HOMO Orbitals . . . . . . . . . . . . . . 58

3.8 Bader Charge-HOMO-LUMO Gap Correlation ～. . . . . . . . . . . . . 62

3.9 No. of Superatomic Electrons-IP/EA Correlation . . . . . . . . . . . . . 63

3.10 No. of Superatomic Electrons-Cluster Core Volume Correlation . . . . . 65

4.1 Transition Metal-Chalcogen Clusters ． . . . . . . . . . . . . . 70

4.2 Band Structures of the Transition Metal-Chalcogen Clusters-Fullerene Compounds . . . . . . . . . . . . . . . . . 75 
4.3 Magnetic Susceptibilities of the Transition Metal-Chalcogen Clusters-Fullerene Compounds . . . . . . . . . . . . . . . . . . 76

$4.4 \mathrm{Ni}_{9} \mathrm{Te}_{6}\left(\mathrm{PEt}_{3}\right)_{8}$ Cluster and the Separately Investigated Substructures . . 78

4.5 PDOS and PLDOS of Tes . . . . . . . . . . . . . . . . . . . . . 79

4.6 Electron Localization Function of Tes $\ldots \ldots \ldots$. . . . . . . . . . . . . . . 80

4.7 PDOS and PLDOS of $\mathrm{CO}_{6} \ldots \ldots \ldots \ldots \ldots$

4.8 PDOS and PLDOS of $\mathrm{Nig} \quad \ldots \ldots \ldots$

4.9 PDOS and PLDOS of $\mathrm{Cr}_{6} \ldots \ldots \ldots \ldots$

4.10 PDOS and PLDOS of $\mathrm{CO}_{6} \mathrm{Se}_{8} \ldots \ldots \ldots \ldots$

4.11 PDOS and PLDOS of $\mathrm{Ni}_{9} \mathrm{Te}_{6} \ldots \ldots \ldots \ldots$

4.12 PDOS of $\mathrm{C}_{6}$

5.1 Gold Cluster- $\mathrm{C}_{60}$ and the Gold Cluster- $\mathrm{B}_{11} \mathrm{H}_{14}$ Dimer . . . . . . . . . . . 95

$5.2 \quad$ PDOS of $\mathrm{B}_{11} \mathrm{H}_{14} \ldots \ldots \ldots \ldots \ldots$

$5.3 \quad \mathrm{Au}_{12} \mathrm{Al}\left(\mathrm{RS}(\mathrm{AuSR})_{2}\right)_{6} \mathrm{C}_{60}$ Rock-Salt Like Bulk Structure $\ldots . . . . .98$

5.4 Band Structure of the $\mathrm{Au}_{12} \mathrm{Al}\left(\mathrm{RS}(\mathrm{AuSR})_{2}\right)_{6} \mathrm{C}_{60}$ Assembly . . . . . . . . 100

5.5 PDOS of the $\mathrm{Au}$ Core in the $\mathrm{Au}_{12} \mathrm{Al}\left(\mathrm{RS}(\mathrm{AuSR})_{2}\right)_{6}-\mathrm{C}_{60}$ Assembly $\ldots .$.

$5.6 \quad \mathrm{Au}_{12} \mathrm{Al}\left(\mathrm{RS}(\mathrm{AuSR})_{2}\right)_{6}-\mathrm{B}_{11} \mathrm{H}_{14}$ Zincblende Like Bulk Structure . . . . . . . 101

5.8 Band Structure of the $\mathrm{B}_{11} \mathrm{H}_{14}-\mathrm{Au}_{12} \mathrm{Al}\left(\mathrm{RS}(\mathrm{AuSR})_{2}\right)_{6}$ Assembly . . . . . . 104

5.9 PDOS of $\mathrm{Si}_{16} \mathrm{Ta} \ldots \ldots \ldots$

$5.10 \mathrm{Au}_{13}\left(\mathrm{RS}(\mathrm{AuSR})_{2}\right)_{6}{ }^{-\mathrm{Si}_{16}} \mathrm{Ta}$ and the $\mathrm{Au}_{13}\left(\mathrm{RS}(\mathrm{AuSR})_{2}\right)_{6}$-Na Dimer $\ldots . . .105$

$5.11 \mathrm{Au}_{12} \mathrm{Al}\left(\mathrm{RS}(\mathrm{AuSR})_{2}\right)_{6}-\mathrm{Au}_{13}\left(\mathrm{RS}(\mathrm{AuSR})_{2}\right)_{6}$ CsI Like Bulk Structure . . . . 108

5.12 Band Structure of the $\mathrm{Au}_{12} \mathrm{Al}\left(\mathrm{RS}(\mathrm{AuSR})_{2}\right)_{6}-\mathrm{Au}_{13}\left(\mathrm{RS}(\mathrm{AuSR})_{2}\right)_{6}$ Assem-

bly . . . . . . . . . . . . . . . . . . . . 109

5.13 PDOS of the $\mathrm{Au}_{12} \mathrm{Al}$ Core in the $\mathrm{Au}_{12} \mathrm{Al}\left(\mathrm{RS}(\mathrm{AuSR})_{2}\right)_{6} \mathrm{Au}_{13}\left(\mathrm{RS}(\mathrm{AuSR})_{2}\right)_{6}$

Assembly . . . . . . . . . . . . . . . . . 110

5.14 Ligand Unprotected $\mathrm{Au}_{12} \mathrm{Al}$ and $\mathrm{Au}_{13}$ cluster Based Bulk Structure . . . 111

5.15 Bader Charge-Cohesive Energy Correlation . . . . . . . . . . . . . . . . 112

5.16 Cohesive Energy-Nearest Neighbor Distance Correlation . . . . . . . . . 113

5.17 Cluster Dimer Energy Decomposition Analysis . . . . . . . . . . . . . . . 114

$6.1 \mathrm{Au}_{13}$ Clusters Protected with Different Ligands . . . . . . . . . . . . 120 
6.2 HOMO Radial Charge Distribution for 3 Clusters with Different Ligands 125

$7.1 \mathrm{Au}_{9}\left(\mathrm{PPh}_{3}\right)_{8}-\mathrm{C}_{60}$ Assemblies $\ldots \ldots \ldots \ldots \ldots \ldots$

7.2 XRD Spectrum for the $\mathrm{Au}_{9}\left(\mathrm{PPh}_{3}\right)_{8}-\mathrm{C}_{60}$ Assembly . . . . . . . . . . . . . 131

7.3 PDOS of $\mathrm{Aug}$. . . . . . . . . . . . . . . . . . . . . . . . . 132

7.4 Band Structures of the $\mathrm{Au}_{9}\left(\mathrm{PPh}_{3}\right)_{8}-\mathrm{C}_{60}$ Assemblies . . . . . . . . . . . . 133

7.5 Aluminium ()xide Clusters . . . . . . . . . . . . . . . 136

7.6 Ligand Positions in Cluster $\mathrm{Al}_{4} \mathrm{~F}_{4}\left(\mu_{4}-\mathrm{O}\right)(\mu-\mathrm{OH})_{5}\left(\mathrm{H}(\mathrm{OH})_{2}\right)$. . . . . . . . 138

7.7 Ligand Positions in Cluster $\mathrm{Al}_{4}\left(\mu_{4}-\mathrm{O}\right)\left(\mu-\mathrm{O}^{\imath} \mathrm{Pr}\right)_{5}\left(\mathrm{H}\left(\mathrm{O}^{\imath} \mathrm{Pr}\right)_{2}\right)\left(\mathrm{O}^{\imath} \mathrm{Pr}\right)_{4}$. . . 140

7.8 Reaction of $\mathrm{Al}_{4} \mathrm{~F}_{4}\left(\mu_{4}-\mathrm{O}\right)(\mu-\mathrm{OH})_{5}\left(\mathrm{O}^{2} \mathrm{Pr}\right)\left(\mathrm{H}_{2} \mathrm{O}\right)$ with $\mathrm{H}_{2} \mathrm{O} \quad \ldots . . . . \quad 141$

7.9 Second Reaction Possibility of $\mathrm{Al}_{4} \mathrm{~F}_{4}\left(\mu_{4}-\mathrm{O}\right)(\mu-\mathrm{OH})_{4}\left(\mu-\mathrm{O}^{i} \mathrm{Pr}\right)_{1}\left[\mathrm{H}(\mathrm{OH})_{2}\right.$. . 142 7.10 Initial Water Molecule Positions . . . . . . . . . . . . . . . . . . . 144

7.11 Binding Energies of the last $\mathrm{H}_{2} \mathrm{O}$ added to $\mathrm{Al}_{4} \mathrm{~F}_{4}\left(\mu_{4}-\mathrm{O}\right)(\mu-\mathrm{OH})_{5}\left(\mathrm{H}(\mathrm{OH})_{2}\right) 144$ 7.12 Binding Energies of the last $\mathrm{H}_{2} \mathrm{O}$ added to $\mathrm{Al}_{4}\left(\mu_{4}-\mathrm{O}\right)(\mu-\mathrm{OH})_{5}\left(\mathrm{H}(\mathrm{OH})_{2}\right)(\mathrm{OH})_{4} 145$ 7.13 Lowest Binding Energies at Cluster $\mathrm{Al}_{4} \mathrm{~F}_{4}\left(\mu_{4}-\mathrm{O}\right)(\mu-\mathrm{OH})_{5}\left(\mathrm{H}(\mathrm{OH})_{2}\right)$. . . . 145 7.14 Lowest Binding Energies at $\mathrm{Al}_{4}\left(\mu_{4}-\mathrm{O}\right)(\mu-\mathrm{OH})_{5}\left(\mathrm{H}(\mathrm{OH})_{2}\right)(\mathrm{OH})_{4} \ldots$. . . . 146 7.15 Water shell around the Aluminum ()xide Clusters . . . . . . . . . . . . . 147 


\section{List of Tables}

$3.1 \quad$ Distortion of the Doped $\mathrm{Au}_{13}$ Cluster Core Structure . . . . . . . . . . . 57

3.2 Bader Charges and HOMO-LUMO gaps of the different $\mathrm{Au}_{13}\left(\mathrm{RS}(\mathrm{AuSR})_{2}\right)_{6}$ Clusters . . . . . . . . . . . . . . . . . . . . . . 61

$3.3 \quad$ IPs and EAs for the Differently Doped Clusters . . . . . . . . . . . . . . 64

4.1 Lattice Parameters of the Transition Metal-Chalcogen Clusters-Fullerene Compounds . . . . . . . . . . . . . . . . . . . 71

4.2 Cohesive Energies and Bader Charges of the Transition Metal-Chalcogen Clusters-Fullerene Compounds . . . . . . . . . . . . . . . . . . . . 72

4.3 Bader Charges of the Transition Metals of the Metal-Chalcogen Clusters 84

5.1 Cation-Anion Radius Ratio and Predicted Binary Solid-State Coordination Environments . . . . . . . . . . . . . . . . . . . . 92

5.2 Cohesive Energies and Bader Charges for Different Cluster Dimers . . . . 95

5.3 Cohesive Energies and Bader Charges of the $\mathrm{Au}_{12} \mathrm{Al}\left(\mathrm{RS}(\mathrm{AuSR})_{2}\right)_{6}-\mathrm{C}_{60}$ Bulk Structure . . . . . . . . . . . . . . . . . . . . . . . . . . . 98

5.4 Cohesive Energies and Bader Charges of the $\mathrm{Au}_{12} \mathrm{Al}\left(\mathrm{RS}(\mathrm{AuSR})_{2}\right)_{6}-\mathrm{B}_{11} \mathrm{H}_{14}$ Bulk Structure . . . . . . . . . . . . . . . . . . . . . . . . . 102

5.5 Cohesive Energies and Bader Charges for the $\mathrm{Au}_{13}\left(\mathrm{RS}(\mathrm{AuSR})_{2}\right)_{6}-\mathrm{Au}_{12} \mathrm{Al}\left(\mathrm{RS}(\mathrm{AuSR})_{2}\right)_{6}$

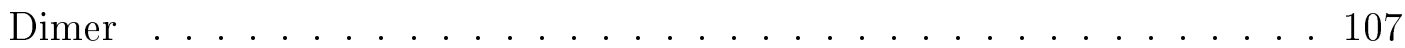

5.6 Cohesive Energies and Bader Charges for the $\mathrm{Au}_{12} \mathrm{Al}\left(\mathrm{RS}(\mathrm{AuSR})_{2}\right)_{6}-\mathrm{Au}_{13}\left(\mathrm{RS}(\mathrm{AuSR})_{2}\right)_{6}$ Bulk Structure . . . . . . . . . . . . . . . . . . . . . . . . . . 108

6.1 Contributions to the Total Interaction Energy for the Clusters Exhibiting Different Ligands . . . . . . . . . . . . . . . . . . . . . . . . . . 121 
6.2 HOMO-LUMO gap values for the $\mathrm{Au}_{13}$ cluster exhibiting different ligands 123

6.3 EA and IP values for the $\mathrm{Au}_{13}$ cluster exhibiting different ligands . . . . 123

6.4 Bader charges for the clusters exhibiting different ligands . . . . . . . . . 124

7.1 Bader Charges for the $\mathrm{Au}_{9}\left(\mathrm{PPh}_{3}\right)_{8}-\mathrm{C}_{60}$ Assemblies . . . . . . . . . . . . . 130

7.2 Reaction Free Energies for the Hydroxylation Reaction . . . . . . . . . . 138

7.3 Reaction Free Energies for the Hydroxylation Reaction along two Paths . 139

7.4 NMR Shifts for the Aluminum ()xide Clusters in Vaculm and Water . . 149 


\section{Introduction}

If a cluster with suitable size and composition could be synthsized such that it mimics the chemistry of an atom in the periodic table, such a cluster could be regarded as a man-made "element" Puru Jena

\subsection{Metal Clusters}

Metal clusters play a crucial role in chemistry. They have been subject to interdisciplinary research for many years as they act as a bridge between atoms and solid-state materials. Their area of application and investigation ranges from coordination chemistry, organic chemistry and catalysis to solid-state chemistry, physics and material sciences. Even though the first metal cluster has been reported in the literature about 110 years ago ${ }^{\text {[ }}$, this field of research is, due to improving techniques and an increasing number of ligands and metals available, still exceptionally active. Hence, it is leading to the creation of a diverse array of promising metal clusters and cluster based materials for various applications.

To further improve and promote this field of research it was and still is necessary to study the fundamental characteristics of metal clusters e.g. atomistic structures, electronic structures as well as chemical and magnetic properties. A considerable number of theoretical and experimental studies conducted on these characteristics show that

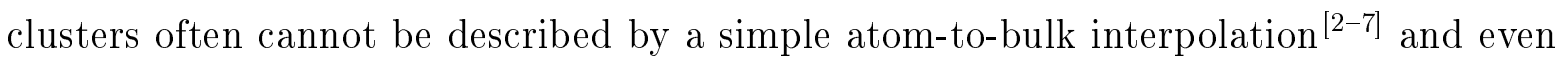
reveal properties that differ significantly from their corresponding bulk materials. An example for the latter is the extraordinary melting behavior of gallium. While bulk gallium is melting at room temperature, the melting temperature of small gallium clusters 
is a few $100 \mathrm{~K}$ higher ${ }^{[0,6,6,8,[]}$. Another example is the frequent use of gold clusters as catalysts, while the bulk is mostly unreactive ${ }^{[11-[3]}$.

In most of these cases quantum confinement effects have to be consulted ${ }^{[14,15]}$ since physical and chemical properties that scale with system size can only sometimes be understood by classical size effects ${ }^{\left[{ }^{[6]}\right]}$. One very outstanding quantum confinement effect is the distinct thermodynamic stability of metal clusters with a very specific number of atoms and their unusual atom like behavior ${ }^{[17,[18]}$. This so-called "superatomic nature" of those nanoparticles lays the foundation for our investigations and will therefore be discussed in detail in the next section.

\subsection{Superatom Model}

The first step towards establishing the superatom model was done in the early 80 s when Knight et al. observed sodium clusters produced through supersonic expansion with argon as carrier gas ${ }^{[10]}$. Time of flight measurements that were undertaken to investigate the resulting cluster mass distribution revealed a high abundance of sodium clusters consisting of $2,8,18,20,34, \ldots$ atoms. Although such an exceptional thermodynamic stability for a specific amount of atoms, the so called "magic numbers", had already been observed before, Knight et al. were the first to recognize that the ordering of those nanoparticles can be explained by applying a shell model to those clusters ${ }^{[[9,20]}$. This procedure is similar to the idea that lead to the well established electronic shell theories of individual atoms and nuclei. These theories however, are both based on the idea that the structures of nuclei and atoms are reasonably well described by assuming an effective single particle spherical potential, even though the underlying Fermion interactions responsible for the structure differ between both systems. Thus, it seems natural to apply a similar model to clusters, here in particular to sodium clusters and to consider the delocalized $3 s^{1}$ valence electrons to be bound in a spherical potential well. Furthermore, in analogy to superatomic clusters, nuclei reveal unusual stability for certain numbers of Fermions, and therefore magic numbers ${ }^{[21-[23]}$, supporting the choice of the cluster description. 
Simultaneously and independently of that finding, Ekardt predicted a similar shell structure for clusters consisting of alkali atoms by using a self-consistent spherical jellium model to calculate absorption spectra for small clusters ${ }^{[24,25]}$. This appeared to be an even more successful approach for the prediction of shell closing numbers, and many other properties of metal clusters.

The jellium model has initially been used for surface/bulk calculations ${ }^{[26-29]}$. The basic idea behind this model is to consider delocalized valence electrons to be moving in a mean field created by the ions. The jellium model also assumes that the lattice structure does not matter and therefore treats the nuclei as a constant positive background charge. The problem of including the not explicitly treated core electrons is usually addressed with pseudopotentials which describe the interaction between the valence electrons and the potential created by core electrons and the nucleus (for a more technical description see chapter [1).

The spherical jellium model however, assumes the ionic background and therefore the cluster to exhibit a spherical potential, thus to mostly be shaped like a sphere. Since jellium model based calculations are usually done within the framework of density functional theory (DFT), which will be explained in detail in chapter 2, starting off with a spherical potential for the ions and additionally assuming the valence electron density to have spherical symmetry, leads to a spherical symmetry of the total Kohm-Sham potential. This results in spherical harmonics as eigenfunctions of the angular part of the single particle states characterized by the quantum numbers $l$ and $m$. Moreover, the Kohn-Sham equations can be reduced to radial Schrödinger equations for the radial parts of the wavefunctions and be solved numerically [30]. The solution yields discrete values for the electronic energy levels characterized by the angular momentum number $l$ reminiscent of individual atoms ${ }^{[10,31]}$. The energetic ordering of those levels for the spherical potential is described by the following Aufbau rule ${ }^{[3]]}$ :

$$
1 S^{2}\left|1 P^{6}\right| 1 D^{10}\left|2 S^{2}, 1 F^{14}\right| 2 P^{6}, 1 G^{18} \mid \ldots
$$

where $S, P, D, F, G$ represent the angular momentum characters of the delocalized states. It has to be noted that capital letters will be used when ever superatomic states are 
referred to. The vertical bars indicate significant energy gaps between different energy levels, and therefore the shell closing numbers, the magic numbers, associated with stable clusters.

The ordering of the energy levels is very sensitive to the shape of the potential, which depends on the cluster shape. This can be seen nicely by considering the variational form of this model where the Kohn-Sham potential is replaced by a potential of a specific shape ${ }^{[32]}$. That the shape of the potential has indeed an impact on the spacing as well as on the ordering of the levels is illustrated in Fig. ㄴ.17. This sensitivity of the energy level

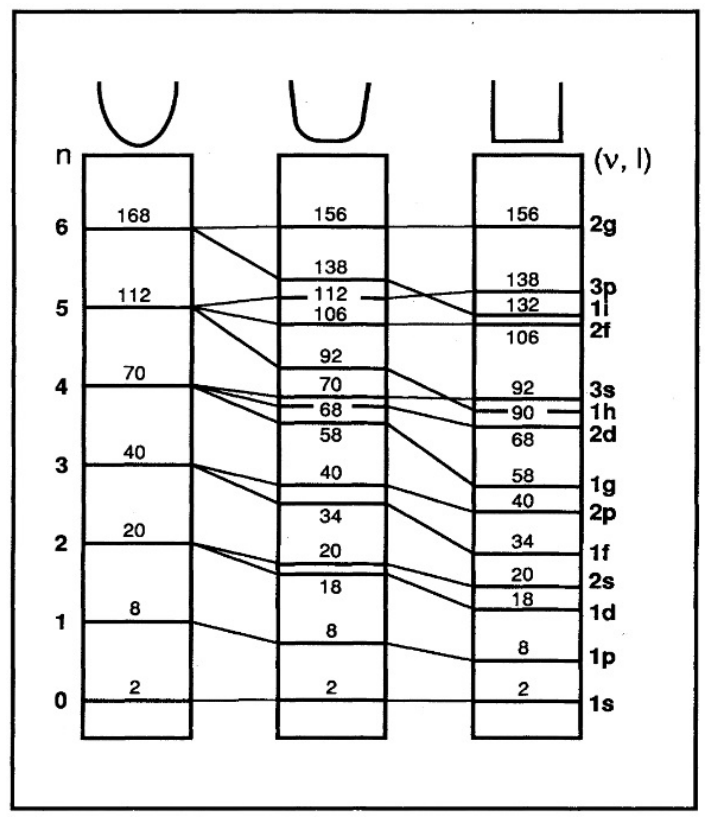

Figure 1.1: Ordering and spacing of the energy levels for three different potentials: harmonic, intermediate and square-well. Reprinted with permission from Ref. ${ }^{[3]}$.

ordering also applies to the model introduced by Ekardt which has become the most used model in the treatment of small metal clusters and has therefore been extended and modified multiple times to appropriately describe different findings.

The preceding passages have already indicated that those metal clusters which show properties reminiscent of an individual atom have come to be referred to as "superatoms" and the self-consistent spherical jellium model as "superatom model". Throughout this thesis, if not indicated otherwise, this therms will be continued to be used in the same 
sense, which additionally involves the idea of the clusters to show characteristics of individual atoms.

\subsection{Establishment and Application of the Superatom Concept}

\subsubsection{Bare Metal Clusters}

Based on the idea that clusters might not only have an electronic structure reminiscent to that of individual atoms but effectively reveal atom-like properties and chemical behavior, further experiments were conducted that lead towards the entrenchment of the superatomic concept. One of the first experiments to show that the superatomic nature has an impact on the cluster reactivity was conducted in 1989. Castleman et al.

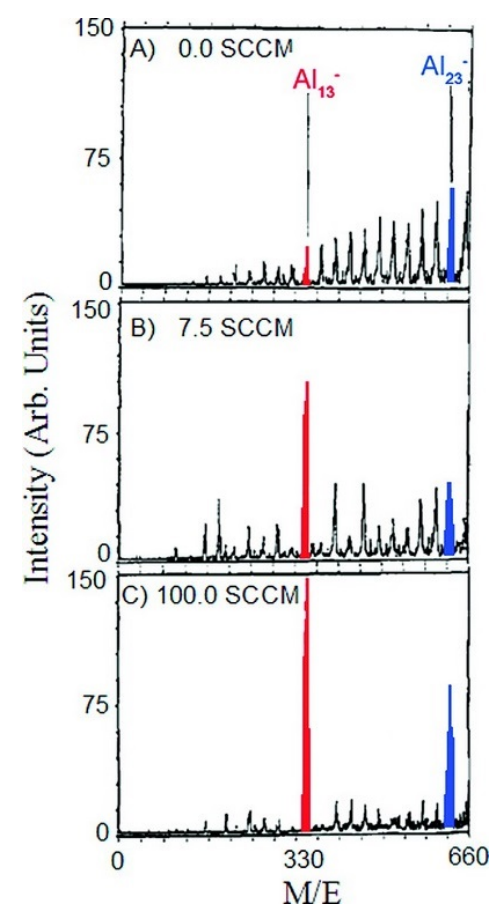

Figure 1.2: Aluminum cluster mass spectra obtained after different oxygen exposures: A) 0.0 sccm oxygen, B) $7.5 \mathrm{sccm}$ and C) $100.0 \mathrm{sccm}$. Reprinted with permission from Ref. [33]. 
investigated the reactivity of aluminum clusters, formed by laser vaporisation, towards oxygen $^{[34]}$. Fig. [12] visualizes the observed mass spectra showing the aluminum cluster distribution with different oxygen pressures. The graphic reveals that most anionic cluster signals disappear due to a reaction with oxygen while clusters containing 13 and 23 atoms do not only stay unreactive but increase in abundance. Again these findings can be explained by applying the jellium shell model. Since aluminum has three valence electrons, the unreactive anionic clusters correspond to shell closing numbers of 40 and 70 superatomic electrons. Thus, superatomic shell closing leads to high stability and low reactivity. The increase in abundance can be explained with oxygen etching of the other clusters until they reach the stable closed shell configurations. Similar work confirming those findings followed, employing aluminum-carbon cluster ${ }^{[35]}$, and niobium or vanadium containing aluminum cluster oxygen etching ${ }^{[36]}$.

The remarkable stability of the $\mathrm{Al}_{13}^{-}$cluster (see Fig. 1.2) and the recently established superatom concept led Khanna and Jena to further investigate this nanoparticle. They concluded that due to its electronic structure $\mathrm{Al}_{13}$ must chemically behave like a halogen. Fig. [..3 shows that similar to halogen atoms the $\mathrm{Al}_{13}$ cluster requires one electron for shell closing. Moreover, this additional electron would lead to a $p / \mathrm{P}$ shell closing in both
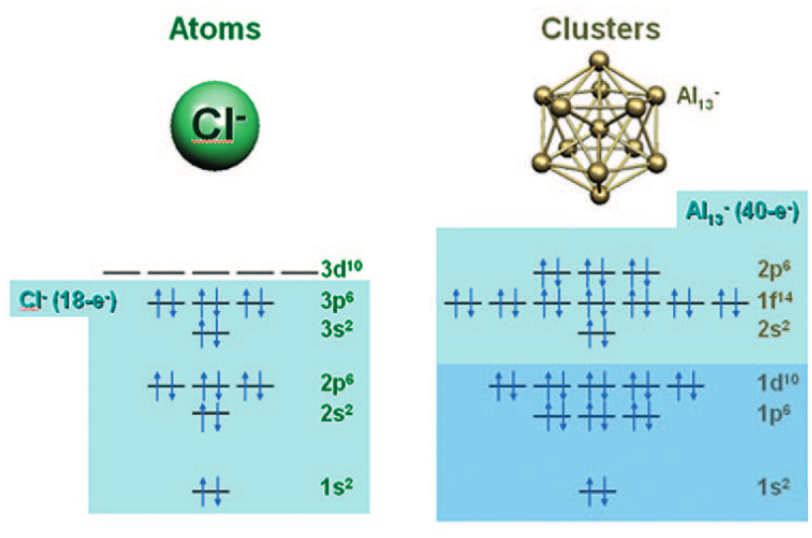

Figure 1.3: Electronic shell structure of an individual chlorine atom and the superatomic $\mathrm{Al}_{13}^{-}$ cluster. Reprinted with permission from Ref. ${ }^{[33]}$.

cases. Having this idea in mind Khanna and Jena calculated the electron affinity of $\mathrm{Al}_{13}$ to be very similar to the electron affinity of an individual chlorine atom ${ }^{[3]}$ and therefore 
gave evidence for the concept that superatomic clusters can mimic the chemistry of individual atoms. Experimental evidence for these findings was given a few years later by e.g. forming polyiodides incorporating the aluminum cluster and therefore showing that the cluster acts similar to an individual iodine atom in polyhalides ${ }^{[17,[18,38]}$. The discovery of a "superhalogen" started a new era, based on the idea, that it should in theory be possible to simulate any element by a superatomic cluster with a similar electronic structure. This led to a considerable amount of work that has been conducted in this field over the past years ${ }^{[39-41]}$. Recently, a lot of attention has focused on designing superatoms with specific properties. The search for interesting magnetic properties has inspired the consideration of early transition metal atom containing clusters. Due to the complexity of the electronic structure of some transition metals, they have been mostly included as dopants or impurities in superatomic clusters build primarily of $s$ or $p$-block metals ${ }^{[42-44]}$. Clusters mainly or solely consisting of complex transition metals are still to be studied in the superatomic sense. A superatomic investigation of selected transition metal containing clusters can be found in chapter $\mathbb{6}$.

\subsubsection{Ligand Protected Metal Clusters}

A lot of work on superatoms focuses on ligand protected clusters, since they can be produced economically and reveal additional stability due to the ligands. The ligands protecting the superatomic cluster core can either act as weak Lewis bases, withdraw electrons out of the delocalized charge or localize them through covalent bonds to the actual metal cluster. Ligand protected magic clusters therefore are those that fulfill the following counting rule ${ }^{[4.5]}$ :

$$
n^{*}=N \nu_{A}-M-z,
$$

where $n^{*}$ are the magic numbers, $N$ is the amount of atoms the cluster core consist of, $\nu_{A}$ is the atomic valency each cluster core atom contributes to the delocalized charge, $\mathrm{M}$ is the amount of electrons localized/withdrawn by the ligands and $z$ the charge of the whole cluster.

Superatomic investigations have been conducted on e.g. ligand protected aluminum, gallium and silver clusters ${ }^{[46-50]}$. However, most publications target ligand protected 
gold clusters due to catalytic reasons and potential application in sensing, molecular electronics and photonics.

Beginning with the late 60s, phosphine ligands have been the most commonly used ligands to protect gold clusters ${ }^{[51-55]}$. This mainly changed in the mid 90 s when Brust et al. published the synthesis of thiolate protected gold nanoparticles that revealed much higher stability arising from a strong bond between the gold atoms and the ligands [56,57]. Although many different synthesis routes have been developed, they all rely on the reduction of Au salts yielding a mixture of clusters of different size, which usually need to be separated in another step.

Due to the complexity of crystallizing ligand protected gold nanoparticles, the structural analysis initially concentrated mainly on theoretical methods, leading to different structure predictions for the same cluster ${ }^{[5-60]}$. The major breakthrough came in 2007 when a complete experimental X-ray structure determination of $\mathrm{Au}_{102}(p-\mathrm{MBA})_{44}(p-\mathrm{MBA}=$ $p$-mercaptobenzoic acid) was published ${ }^{[6]]}$. Fig. $\mathbb{L . 4}$ is based on further theoretical

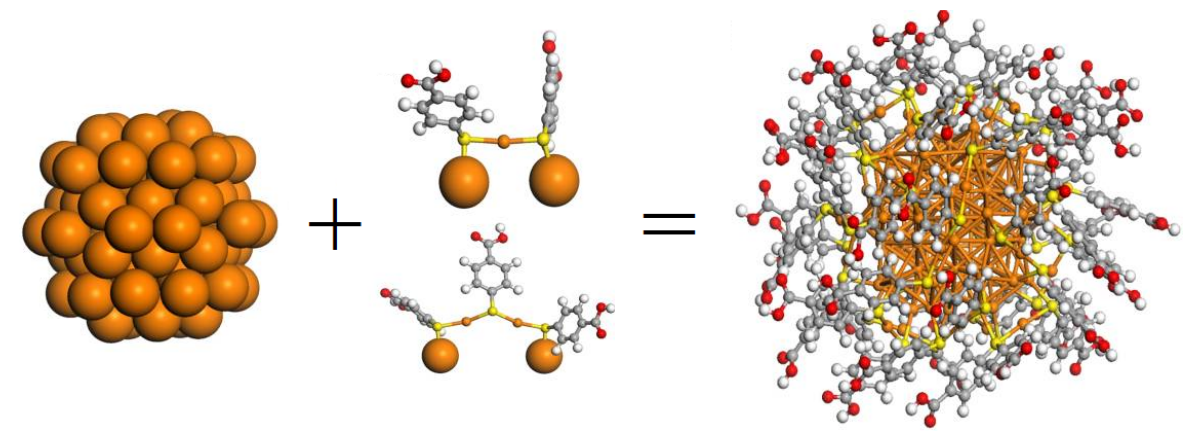

Figure 1.4: Visualization of the $\mathrm{Au}_{102}(p-\mathrm{MBA})_{44}$ cluster. Left image: "naked" $\mathrm{Au}_{79}$ core, middle: two different types of ligands protecting the cluster, picture on the right: structure of the corresponding ligand protected cluster. Color code: Au: orange, S: yellow, O: red, C: gray and H: white. Reprinted with permission from Ref. ${ }^{[62]}$.

analysis of the $\mathrm{Au}_{102}(p-\mathrm{MBA})_{44}$ cluster and reveals that it consists of an approximately $D_{5 h}$-symmetric cluster core which is protected by two different types of ligands. The most abundant ligand visualized in the upper part of Fig. 1.4 consists of a RS-Au-SR unit whereas the other ligand consisting of a RS-Au-SR-Au-SR unit, solely appears twice in the cluster structure [62]. Furthermore the calculated HOMO-LUMO gap of the cluster was found to be $0.5 \mathrm{eV}$ indicating a very stable species and therefore a superatomic shell 
closing. The investigations revealed that the ligand sulfur atoms which are located close to the gold core form covalent bonds with the gold cluster core surface atoms. This leads to a localization of 21 gold valence electrons, thus those electrons can not contribute to the superatomic charge, which is resulting in an overall 58 superatomic electron count and therefore shell closing ${ }^{[61,62]}$. In addition Häkkinen et al. confirmed the shell closing and the metallic character by looking at the charge distribution of the anionic and cationic clusters, where $90 \%$ of the charge was found to be located at the ligands and $10 \%$ around the cluster core ${ }^{[\mathrm{[5}]}$.

Since the structure determination of $\mathrm{Au}_{102}(p-\mathrm{MBA})_{44}$, many other thiolate protected gold clusters that are known to be superatomic have been characterized experimentally ${ }^{[63-67]}$ and/or analyzed theoretically ${ }^{[67-[0]}$. Another very important finding was made shortly after. The crystallographic structure of $\mathrm{Au}_{25}(\mathrm{SR})_{18}^{-}$was reported by Murray et al. ${ }^{[7]]}$. This cluster has been known to be one of the smallest, highly stable thiolate protected gold clusters for many years and is furthermore isolatable with good monodispersity, which makes it a very interesting target for both theoretical and experimental studies. For the crystallization process Murray co-workers used $\mathrm{SCH}_{2} \mathrm{CH}_{2} \mathrm{Ph}$ as ligand and $\mathrm{N}\left(\mathrm{C}_{8} \mathrm{H}_{17}\right)_{4}^{+}$as counterion. The crystallization occurred in a 1:1 counterion to cluster stochiometry pointing out that the cluster prefers to be singly charged.

Fig. 1.5 shows that this nanoparticle consists of an approximately icosahedral $\mathrm{Au}_{13}$ cage protected by only one type of ligand, namely six RS-Au-SR-Au-SR units. Every gold core atom is in theory providing one $6 s$ electron to the delocalized electron shell, while the ligands are each localizing one of those electrons. Considering the additional charge, this results in a total electron count of eight delocalized electrons, which corresponds to a $1 \mathrm{~S}^{2} 1 \mathrm{P}^{6}$ shell closing. The angular momentum projected density of states analysis close to the HOMO-LUMO gap confirms the superatomic shell closing since the HOMO state is P like while the LUMO state is mostly D like.

To further explore the structure and chemical and physical properties of $\mathrm{Au}_{25}(\mathrm{SR})_{18}^{-}$optical absorption experiments, matching calculations ${ }^{[03]}$ and femtosecond two-dimensional electronic spectroscopy experiments were conducted ${ }^{[74]}$. The obtained spectra very closely matched the predicted superatomic electronic structure by showing single electron transitions between discrete molecule like states which is in contrast to the metal 

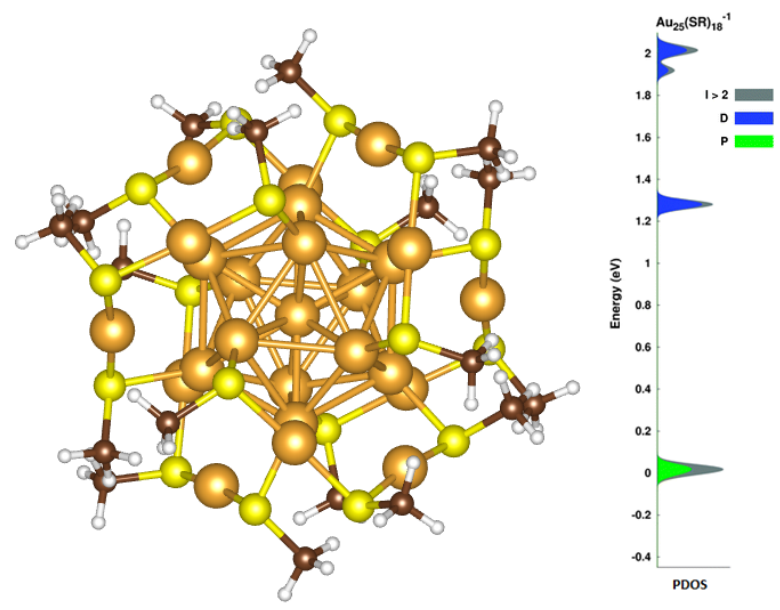

Figure 1.5: Schematic representation of the $\mathrm{Au}_{25}\left(\mathrm{SCH}_{2} \mathrm{CH}_{2} \mathrm{Ph}\right)_{18}^{-}$cluster with reduced ligands (replacing the $\mathrm{CH}_{2} \mathrm{CH}_{2} \mathrm{Ph}$ units by $\mathrm{CH}_{3}$ ) and the angular momentum projected density of states obtained for the visualized cluster reprinted with permission from Ref. ${ }^{[[2]}$. Color code: Au: yellow, S: orange, C: gray and H: white.

like spectra obtained for larger clusters. The latter technique furthermore resolved the electron dynamics of the superatomic P and D states. Beyond that the fluorescence of small gold nanoparticles, in particular the $\mathrm{Au}_{25}(\mathrm{SR})_{18}^{-}$cluster, has been observed and found to be an interesting property for potential application to biosensors ${ }^{[75]}$. Although the fluorescence mechanism has not been completely understood yet, two possible sources have been proposed. First, a metal centered electron transition again relying on discrete molecule like states and second a fluorescence caused by the interaction between the cluster core and the ligands ${ }^{[76]}$.

In contrast to diamagnetic gold bulk and paramagnetic individual gold atoms, gold clusters can reveal both forms of magnetism. The $\mathrm{Au}_{25}(\mathrm{SR})_{18}^{-}$cluster can be oxidized chemically leading from a diamagnetic cluster to a paramagnetic $\mathrm{Au}_{25}(\mathrm{SR})_{18}^{0}$ and a diamagnetic $\mathrm{Au}_{25}(\mathrm{SR})_{18}^{+}$cluster ${ }^{[\mathrm{[7},[\mathrm{Br}]}$. Jin et al. additionally showed that an oxidation of the paramagnetic cluster affects the cluster core, which contradicts the previous assumption that the thiolate ligands are responsible for the magnetism due to a charge transfer occurring between the core and ligands ${ }^{[77]}$. This finding again points out the strong superatomic character of the $\mathrm{Au}_{25}(\mathrm{SR})_{18}^{-}$cluster.

Recently a lot of attention was drawn towards doped, thiolate protected superatomic gold 
clusters as synthesized by Fields-Zinna et al. ${ }^{[0]}$ and Negishi et al. ${ }^{[01-\varangle 3]}$. The dopants have opened up the possibility to influence the structural and electronic properties of those clusters, which have therefore been analyzed, both experimentally ${ }^{[84-87]}$ and theoretically $[02,88-90]$. The tunability of the electronic structure of the superatomic cluster is a central point when it comes to considering what these clusters can be used for and will therefore be discussed extensively within this thesis.

While a lot of work has been done regarding the $\mathrm{Au}_{25}(\mathrm{SR})_{18}^{-}$cluster many other gold clusters fulfilling the magic number counting rule have been synthesized and character-

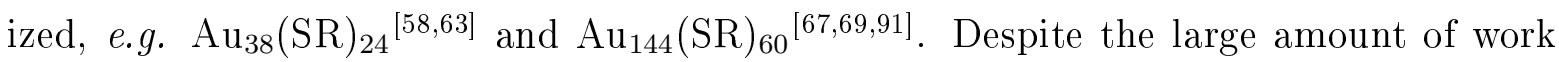
that has already been conducted and published in this field of research, a lot of work still remains to be done in the future, in particular, to bridge the gap in knowledge between these specific examples and other families of metal clusters, which may also be superatomic.

\subsubsection{Superatomic Clusters as new Building Blocks}

As already mentioned in the sections before, clusters behaving as individual atoms and mimicking the chemistry of specific elements leads to the idea of using those nanoparticles as building blocks for new materials. One could raise the question why clusters represent attractive building blocks when a large variety of solid-state materials consisting of individual atoms is available. A quite obvious reason is that the properties of atom based materials are hard to change whereas in the case of nanoparticles shorter length scales need to be considered. To change the properties of cluster assembled materials one solely needs to change the properties of the individual clusters involved. Furthermore, it may be possible to create materials that reveal more than one functionality. To prevent the clusters from coalescing it is important to chose stable clusters. These clusters can be further stabilized through the involvement of ligands which can moreover have a positive effect on the ability of the clusters to self-assemble. One example is the tunable support of superatomic orbitals which are further extended over the whole cluster including the ligands ${ }^{[89]}$. Therefore it is indispensable to investigate the role of the ligands, which will

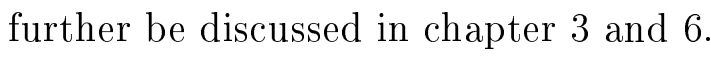


Since designing methods to create specific cluster-assembled materials often remains very challenging, only a few materials have been synthesized ${ }^{[\mathrm{G2}-[\mathrm{W0}]}$. Fig [1.6] shows an example of a series of binary "superlattices" with $\mathrm{AB}, \mathrm{AB}_{2}, \mathrm{AB}_{3}, \mathrm{AB}_{4}, \mathrm{AB}_{5}, \mathrm{AB}_{6}$ and $\mathrm{AB}_{13}$ stoichiometry, that have been created using small clusters stabilized by organic ligands ${ }^{[08]}$. In the visualized example, TEM pictures of different binary cluster-cluster assemblies

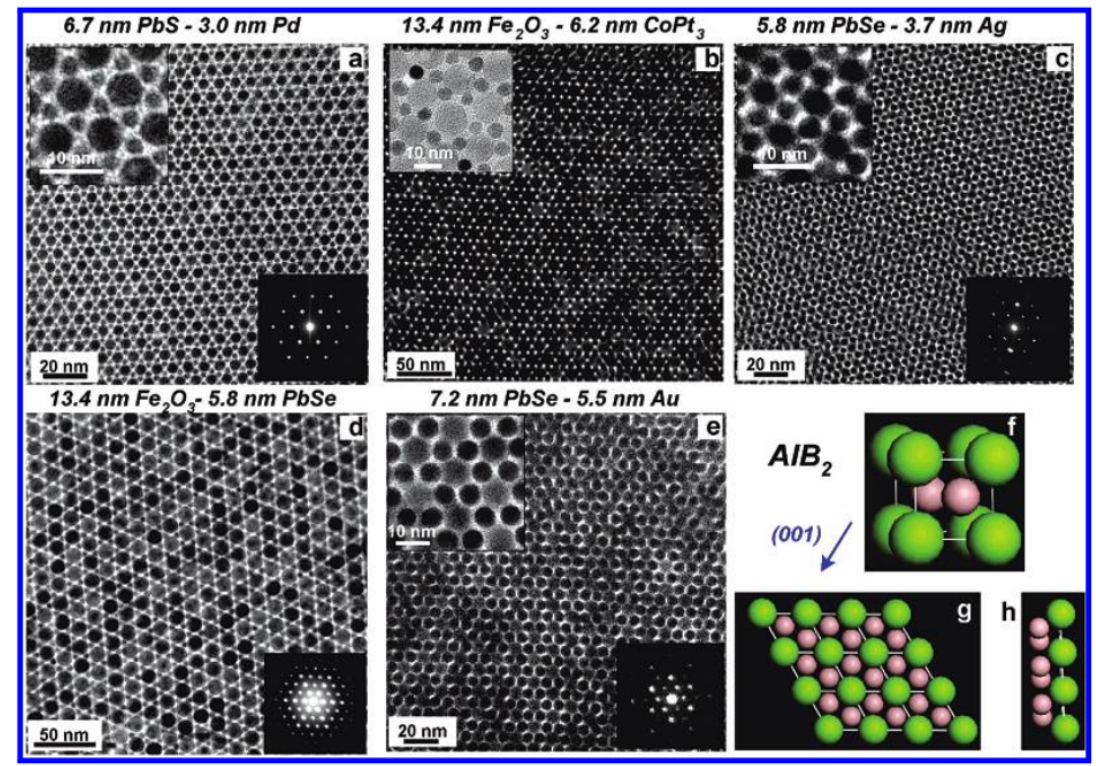

Figure 1.6: TEM pictures of the (001) plane of binary cluster assembled materials assembling in a $\mathrm{AIB}_{2}$-type structure. In the case of every TEM picture the large and small particles used are shown above. The picture on the left depicts the $\mathrm{AIB}_{2}$-type unit cell and the general structure of the (001) plane. Reprinted with permission from Ref. ${ }^{[98]}$.

forming $\mathrm{AIB}_{2}$-type lattices can be seen. This experiment conducted by Shevchenko et al. showed that nearly every possible lattice type can be built up by clusters and furthermore that the particle size ratio of the two components plays a central role as it is the case in atom based lattices.

Another interesting finding was made in 2013, when Roy et al. published the synthesis and characterization of three solid-state materials formed out of an binary assembly of ligand protected clusters and fullerenes ${ }^{[06]}$. The solids containing the electron rich superatomic clusters $\mathrm{Co}_{6} \mathrm{Se}_{8}\left(\mathrm{PEt}_{3}\right)_{6}$ and $\mathrm{Cr}_{6} \mathrm{Te}_{8}\left(\mathrm{PEt}_{3}\right)_{6}$ assembled into a $\mathrm{Cd}_{2} \mathrm{I}$ type structure, providing charge for the electron-poor fullerene. $\mathrm{Ni}_{9} \mathrm{Te}_{6}\left(\mathrm{PEt}_{3}\right)_{8}$ transfers even more 
charge to the fullerene resulting in a rock-salt like assembly of both components with a 1:1 stoichiometry (see Fig. 4.7). Due to the charge transfer an activated electron
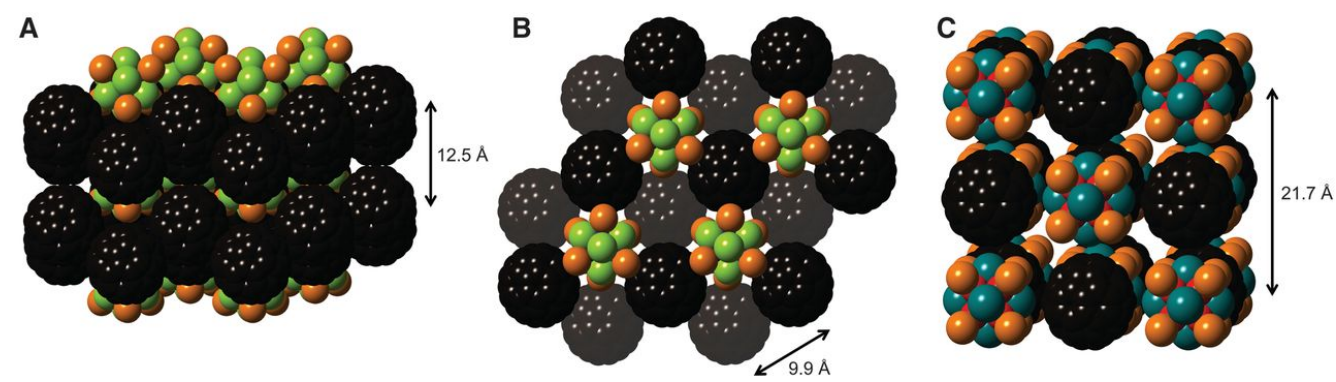

Figure 1.7: Visualization of the solid-state materials created by an binary assembly of superatomic clusters and fullerenes. Picture $\mathrm{A}$ and $\mathrm{B}$ show the structure created by $\mathrm{Co}_{6} \mathrm{Se}_{8}\left(\mathrm{PEt}_{3}\right)_{6}$ and $\mathrm{Cr}_{6} \mathrm{Te}_{8}\left(\mathrm{PEt}_{3}\right)_{6}$, while picture $\mathrm{C}$ reveals the structure formed using $\mathrm{Ni}_{9} \mathrm{Te}_{6}\left(\mathrm{PEt}_{3}\right)_{8}$. Reprinted with permission from Ref. [96]].

transport over the whole material could be found in all cases. Moreover interesting magnetic properties were found. Both properties make the materials, as well as the single clusters, interesting targets for further investigation into how the superatomic character of the individual clusters gives rise to the properties of the corresponding solids and will therefore be discussed in chapter 6 .

\subsection{Limits of the Superatom Model}

The superatom theory has succeeded in explaining many phenomena. However, the model exhibits some weaknesses and limits, when applied to chemically more complex systems. One major problem is that the classical model relies on the jellium treatment of the ionic cores as a uniform background charge, where the individual positions of the nuclei do not matter. This restriction can lead to problems in, for example, describing the superatomic character of doped or bimetallic clusters, since the size and the structure of a cluster changes with the introduction of heteroatoms. Furthermore it is known that the accurate treatment of the electron affinities, the polarizabilities, and the ionization potentials depend on the atomic positions ${ }^{[30]}$.

Several problem specific attempts were undertaken to schematically include some ef- 
fects of the ionic structure by still employing the simple jellium approach while the main general extension has emerged to be the partial inclusion of the ionic structure via pseudopotentials $[27,30,101,102]$. This extension is what is referred to when speaking of the "classical model" or the "jellium model" in this manuscript.

Another restriction of the classical superatom model, as initially introduced by Ekardt and Beck, is the radial shape of the potential. The ordering and spacing of the energetic levels are very sensitive to the potential in which the valence electrons move. For clusters consisting of different types of atoms and not exhibiting an icosahedral (nearly spherical) structure such as $\mathrm{Cr}_{6} \mathrm{Te}_{8}\left(\mathrm{PEt}_{3}\right)_{6}$ (see Fig. L.7), it could be necessary to perturb the potential even more, or even assume a potential with a different shape to achieve the correct ordering of the superatomic energy levels.

Beyond the density functional approach several research groups employed wave-function based methods for the accurate description of superatomic clusters to e.g. evaluate long-range correlation. However the DFT jellium approach, not least due to the computational effort required, still remains the main method of choice when dealing with superatomic systems.

\subsection{Non-Superatomic Custers}

Besides the broad range of different clusters revealing superatomic nature there are clusters that do not exhibit an atom like electron shell structure and nevertheless are important for similar applications and therefore also need to be investigated. Those clusters often have structures that can be described as fragments of the corresponding bulk crystal structure.

Metal oxides can often be regarded as clusters that do not reveal a superatomic nature due to their strong ionic character. Furthermore the stability of those clusters does not follow magic numbers. One central application of metal oxide clusters for industrial purposes is catalysis. In this particular field metal (hydr)oxofluorides used to play a minor role. This mainly changed in 2003 when Kemnitz et al. indroduced the so called "fluorolytic sol-gel synthesis" that gave access to previously inaccessible metal fluorides and metal (hydr)oxofluorides ${ }^{[103-[10 .]}$. The first reaction step of the fluorolytic sol-gel 
synthesis is very similar to the first step of the aqueous sol-gel synthesis thats leads to the formation of M-OH groups and, in a next step, to nanoscale metal oxides. The main difference is that water as reactant is replaced by hydrogen fluoride leading to the following reaction:

$$
\mathrm{M}-\mathrm{OR}+\mathrm{H}-\mathrm{F} \rightarrow \mathrm{M}-\mathrm{F}+\mathrm{ROH}
$$

In a second step the strong bridging tendency of fluoride ions causes the formation of nanoparticles and bulk materials.

Within the framework of a project funded by the EU and the DFG the fluorolytic sol-gel and other methods are utilized to synthesize new metal fluoride/ metal (hydr)oxofluoride catalysts to investigate them as potential catalyst for various reactions like alkylation and isomerization reactions. Furthermore these novel compounds can be used as support for other active compounds like gold or platinum. Beyond that, organo functionalized metal fluorides with low refraction index are synthesized and studied in order to produce new antireflective layer systems. In order to support the experimental studies and to help to understand chemical and physical properties to fully understand the catalytic behavior of those catalysts theoretical studies are conducted by the research group of Prof. Paulus in Berlin.

Section 7.2 of this thesis is focusing on the investigation of the hydroxylated analog of the aluminum (hydr)oxide fluoride cluster that has been synthesized and crystallized by research group of Prof. Kemnitz ${ }^{[06]}$. Even though small aluminum clusters are know to be superatomic, the investigate aluminum oxide have a strong ionic nature. The oxygen localizes the $s$ electrons of the aluminum atoms leading to the loss of superatomic nature. A similar effect between transition metals and chalcogen atoms will be discussed

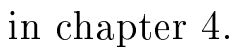

\subsection{Summary}

This work intends to investigate different kinds of superatomic clusters consisting of noble metals and transition metals, clusters containing dopants, important building blocks such as ligands and superatomic compounds to contribute to the overall understanding 
of superatoms. Fundamental research of this kind is essential to gain the knowledge that is needed to target possible applications, as very stable and atom like nanoparticles superatoms seem to be very promising for catalytic application and building blocks for new functional materials.

The research part of this thesis is structured as follows:

\section{Tunability of Superatoms}

In this chapter the superatomic approach is used to computationally analyze the electronic structure of thiolate ligand protected gold clusters to compare between monometallic superatomic clusters and their doped species and to show the effect of the doping on the superatomic structure. Furthermore those inhomogeneities are used to critically evaluate the tunability of the superatomic character of the regarded clusters.

\section{Transition Metal Cluster Assemblies}

In addition to the noble metal cluster study, this chapter discusses the superatom character of transition metal chalcogen clusters and compounds created by a binary cluster-fullerene assembly as synthesized by Roy et al. It is shown that this particular clusters do not exhibit a tunable electronic structure and furthermore lose superatomic character due to a charge transfer occurring between the transition metal and chalcogen atoms.

\section{Screening of Potential Superatomic Compounds}

Here the knowledge gained from the first two chapters is is used to target suitable building blocks for new functional materials based on the tunable gold clusters. The investigation reveals some principles regarding cluster assemblies.

\section{Ligands Protecting the Clusters}

In order to create a complete picture, this chapter discusses the role of the ligands on the stability of gold superatomic clusters. Thus, the importance of the choice of ligand when creating superatomic assemblies.

\section{Experimental Collaborations}

The last results chapter is focused on experimental collaborations stemming from 
the fundamental work of the first 4 results chapters. On one hand the structural and electronic analysis of a experimentally synthesized material consisting of $\mathrm{C}_{60}$ and the superatomic phosphine protected $\mathrm{Au}_{9}$ cluster is discussed. The second project however, is dealing with non-superatomic clusters and therefore reflecting the opposite case. It demonstrates the investigation of the reaction mechanism leading from $\mathrm{Al}_{4} \mathrm{~F}_{4}\left(\mu_{4}-\mathrm{O}\right)\left(\mu-\mathrm{O}^{i} \mathrm{Pr}\right)_{5}\left[\mathrm{H}\left(\mathrm{O}^{i} \mathrm{Pr}\right)_{2}\right]$ to $\mathrm{Al}_{4} \mathrm{~F}_{4}\left(\mu_{4}-\mathrm{O}\right)(\mu-$ $\mathrm{OH})_{5}\left[\mathrm{H}(\mathrm{OH})_{2}\right]$ and furthermore the water absorption on the latter cluster while considering the role of the fluorine atoms. 


\section{Theory}

This chapter aims to introduce the reader briefly to the theoretical background and the methods that were used within the present thesis.

\subsection{The Schrödinger Equation}

The 20th century started a new era of physics. New concepts such as the energy quantization, introduced by Planck to describe black body radiation, [ய]] and the wave-particle duality initially formulated by de Broglie ${ }^{[018]}$ led to a change of thinking, and in the following years to the establishment of quantum physics.

In 1926 Schrödinger contributed by suggesting to utilize the wave function $\Psi$ to describe the state of multiple particle systems ${ }^{[10-\square]}$. $\Psi$ includes all information about the observed system and is therefore a function of all particle space coordinates $\mathbf{r}$, the spin coordinates $\omega$ and the time $t$. Furthermore he set up an equation to calculate the time dependent total energy of a system, which is known as the Schrödinger equation

$$
\hat{H} \Psi=i \hbar \frac{\delta \Psi}{\delta t}
$$

where $\hat{H}$ is the hamilton operator. The probability to find an electron located in volume element $\mathbf{r}+\mathrm{d} \mathbf{r}$ at the time $t$ is according to Born's law $\rho(\mathbf{r}, t)$

$$
\rho(\mathbf{r}, t)=\int|\Psi(\mathbf{x}, t)|^{2} \mathrm{~d} \mathbf{x}_{1} \mathrm{~d} \mathbf{x}_{2} \ldots \mathrm{d} \mathbf{x}_{\mathrm{N}}
$$

with $\mathbf{x}$ denoting the spatial coordinate $\mathbf{r}$ as well as the spin coordinate $\omega$ of each electron. Therefore $\Psi$ is chosen to be normalized such that the probability to find an electron somewhere in the whole volume is exactly one $e^{[13, \pi 14]}$. 
When it comes to solving the Schrödinger equation for a system of interest a few approximations can be employed. A very common simplification is to exclude time dependency and relativity. Therefore the wave function is seperated into a product of a function of time and spatial coordinates yielding the time independent Schrödinger equation

$$
\hat{H} \Psi=E \Psi .
$$

The non-relativistic Hamilton operator corresponds to the classical total energy of the observed system. In the case of a molecule $\hat{H}$ (in atomic units) can be written as,

$$
\begin{aligned}
\hat{H} & =\hat{T}_{\mathrm{e}}+\hat{T}_{\mathrm{n}}+\hat{V}_{\mathrm{ne}}+\hat{V}_{\mathrm{ee}}+\hat{V}_{\mathrm{nn}} \\
& =-\frac{1}{2} \sum_{i}^{N} \nabla_{i}^{2}-\frac{1}{2} \sum_{A}^{M} \nabla_{A}^{2}-\sum_{i}^{N} \sum_{A}^{M} \frac{Z_{A}}{\mathbf{r}_{i A}}-\sum_{i}^{N} \sum_{j}^{N} \frac{1}{\mathbf{r}_{i j}}-\sum_{A}^{M} \sum_{B}^{M} \frac{Z_{A} Z_{B}}{\mathbf{R}_{A B}},
\end{aligned}
$$

with $i$ as the number of electrons, $M$ as the number nuclei and $Z$ as the respective charge. $\quad \mathbf{r}_{i A}$ denotes the distance between electron $i$ and nucleus $A, \mathbf{r}_{i j}$ the distance between electron $i$ and electron $j$, whereas $\mathbf{R}_{A B}$ is the equivalent for nucleus $A$ and nucleus $B$. Thus, the operator $\hat{T}_{\mathrm{e}}$ corresponds to the kinetic energy of the electrons, $\hat{T}_{\mathrm{n}}$ to the kinetic energy of the nuclei, $\hat{V}_{\text {ee }}$ is an operator corresponding to the electrostatic interaction between electrons, $\hat{V}_{\text {ne }}$ between electrons and nuclei and $\hat{V}_{\text {nn }}$ between the nuclei.

Due to the fact that even the non-relativistic Schrödinger equation can only be solved exactly for a few systems such as the hydrogen atom, further approximations are needed. The so called Born-Oppenheimer approximation ${ }^{[15]}$ is based on the idea that molecular systems exhibit two distinct classes of particles, the highly mobile electrons and the nuclei which have a much higher mass and can therefore be seen as stationary, relative to the electrons. Subsequently, the kinetic energy of the nuclei can be neglected and $\hat{V}_{\text {nn }}$ can be treated as a constant. This leads to the simplified electronic Schrödinger equation,

$$
\hat{H}=\hat{T}_{\mathrm{e}}+\hat{V}_{\mathrm{ne}}+\hat{V}_{\mathrm{ee}}+V_{\mathrm{nn}},
$$


where the wave function is a function of the electronic coordinates only. As mentioned at the beginning of the chapter the total wave function does not only include the spatial, but also the spin coordinates of each electron. The spin functions are orthonormalized (see eq. 2.8 in Dirac notation) and can take the values $+\frac{1}{2}(\alpha)$ or $-\frac{1}{2}(\beta)$ which are defined by the alignment of the spin with and opposite to an external magnetic field.

$$
\begin{aligned}
& \langle\alpha \mid \alpha\rangle=\langle\beta \mid \beta\rangle=1 \\
& \langle\alpha \mid \beta\rangle=\langle\beta \mid \alpha\rangle=0 .
\end{aligned}
$$

Moreover electrons with the same spin are indistinguishable. However due to the Pauli exclusion principle an interchange of two identical fermions must change the sign of the wave function.

$$
\Psi\left(\mathbf{x}_{1}, \mathbf{x}_{2}, \ldots, \mathbf{x}_{N}\right)=-\Psi\left(\mathbf{x}_{2}, \mathbf{x}_{1}, \ldots, \mathbf{x}_{N}\right)
$$

The first Ansatz to formulate the total wave function of a molecular system including both the spin and spatial coordinates was done with the Hartree-Product ${ }^{[16]}$ which, however violates the antisymmetry principle. To account for that problem, Slater proposed to write the total wave function of an $N$-electron system as an antisymmetric product of $N$ orthonormalized one electron spin orbitals $\chi_{i}(\mathbf{x})$ referred to as a Slaterdeterminant [17]

$$
\Phi_{\mathrm{SD}}=\frac{1}{\sqrt{N !}}\left|\begin{array}{cccc}
\chi_{1}\left(\mathbf{x}_{1}\right) & \chi_{2}\left(\mathbf{x}_{1}\right) & \ldots & \chi_{n}\left(\mathbf{x}_{1}\right) \\
\chi_{1}\left(\mathbf{x}_{2}\right) & \chi_{2}\left(\mathbf{x}_{2}\right) & \ldots & \chi_{n}\left(\mathbf{x}_{2}\right) \\
\vdots & \vdots & \ddots & \vdots \\
\chi_{1}\left(\mathbf{x}_{N}\right) & \chi_{2}\left(\mathbf{x}_{N}\right) & \ldots & \chi_{n}\left(\mathbf{x}_{N}\right)
\end{array}\right|
$$

The spin orbitals are simply linear combinations of the spatial orbitals $\phi_{i}$ and the spinfunctions $\alpha(\omega)$ or $\beta(\omega)$ thus, every set of $M$ spatial orbitals can be written as a set of $2 M$ spin orbitals

$$
\left.\begin{array}{rl}
\chi_{2 i-1} & =\phi_{i}(\mathbf{r}) \alpha(\omega) \\
\chi_{2 i} & =\phi_{i}(\mathbf{r}) \beta(\omega)
\end{array}\right\} \quad i=1,2, \ldots, M
$$

The Slater-determinant fully accounts for the electron exchange, however the electron 
correlation remains one of the main limitations of the Slater-determinant and thus of the Hartree-Fock method disussed in the next section.

\subsection{Hartree-Fock}

In 1928 Hartree and Fock introduced a method to approximately calculate the wave function and stationary ground state energy of a multiple particle system ${ }^{[\square 8]}$. The method employs a single Slater-determinant as wave function and the Hartree-Fock (HF) equations are solved iteratively according to the variational principle. Thereby the electron-electron interaction problem is addressed by a mean field approximation postulating that each electron moves in an average field created by the other electrons. The variational principle states that the ground state energy $E$ for every trial wave function $\Psi$ can only be greater or equal to the exact ground state energy $E_{0}$

$$
E=\langle\Psi|\hat{H}| \Psi\rangle \geq E_{0}
$$

where the trial wave function $\Psi$ is expressed by a single Slater-determinant and $\hat{H}$ depicts the electronic Hamiltonian (see eq. [2.6), which can be separated into two parts

$$
\begin{aligned}
& \hat{H}=\hat{O}_{1}+\hat{O}_{2} \\
& \hat{O}_{1}=\sum_{i}^{N} \hat{h}=-\frac{1}{2} \sum_{i}^{N} \nabla_{i}^{2}-\sum_{i}^{N} \sum_{A}^{M} \frac{Z_{A}}{\mathbf{r}_{i A}} \\
& \hat{O}_{2}=\sum_{i}^{N} \sum_{j>i}^{N} \frac{1}{r_{i j}}=\sum_{i<j}^{N} \frac{1}{\mathbf{r}_{i j}} .
\end{aligned}
$$


The first term corresponds to the single electron Operator $\hat{O}_{1}$ whereas the second term corresponds to the two electron operator $\hat{O}_{2}$. Applying the Hamilton operator on the Slater-determinant leads to the following notation of the energy expectation value

$$
\begin{aligned}
E= & \sum_{i}^{N}\left\langle\chi_{i}\left(\mathbf{x}_{1}\right)\left|\hat{h}_{i}\right| \chi_{i}\left(\mathbf{x}_{1}\right)\right\rangle+\frac{1}{2} \sum_{i j}^{N}\left(\left\langle\chi_{i}\left(\mathbf{x}_{1}\right) \chi_{i}\left(\mathbf{x}_{2}\right)\left|\frac{1}{\mathbf{r}_{i j}}\right| \chi_{j}\left(\mathbf{x}_{1}\right) \chi_{j}\left(\mathbf{x}_{2}\right)\right\rangle\right. \\
& \left.-\left\langle\chi_{i}\left(\mathbf{x}_{1}\right) \chi_{j}\left(\mathbf{x}_{2}\right)\left|\frac{1}{r_{i j}}\right| \chi_{j}\left(\mathbf{x}_{1}\right) \chi_{i}\left(\mathbf{x}_{2}\right)\right\rangle\right)+V_{n n},
\end{aligned}
$$

where the first term expresses the kinetic energy and electron-nucleus attraction contribution and the last two terms the Coulomb and Exchange integrals respectively. Since the Hartree-Fock energy is a function of the spin orbitals $\chi_{i / j}$ the variational freedom lies in the choice of the orbitals. They are, however, constrained to remain orthonormal which can be satisfied by employing the Lagrange multipliers $\epsilon_{i}$ leading to the following equations known as the Hartree-Fock equations:

$$
\hat{f}_{i}\left|\chi_{i}\right\rangle=\left[\hat{h}_{i}+\sum_{j \neq i}^{N}\left(\hat{J}_{j}-\hat{K}_{j}\right)\right]\left|\chi_{i}\right\rangle=\epsilon_{i}\left|\chi_{i}\right\rangle,
$$

where $\epsilon_{i}$ can be interpreted as the orbital energies and $\hat{f}$ is an effective one electron energy operator namely the "Fock operator". The two electron term $\sum_{j}^{N}\left(\hat{J}_{j}-\hat{K}_{j}\right)$ is nothing else then the Hartree-Fock potential simplifying the complex two electron repulsion operator by replacing it with an average potential. $\hat{J}$ represents the Coulomb operator that can be written in its form as applied to the orbitals as

$$
\hat{J}_{i}\left(\mathbf{x}_{1}\right) \chi_{i}\left(\mathbf{x}_{1}\right)=\left[\int \frac{\left|\chi_{j}\left(\mathbf{x}_{2}\right)\right|^{2}}{\mathbf{r}_{i j}} \mathrm{~d} \mathbf{x}_{2}\right] \chi_{\mathrm{i}}\left(\mathbf{x}_{1}\right)
$$

where the potential experienced by electron one at $\mathbf{x}_{1}$, is calculated by integrating the probability of the presence of electron two over $\mathbf{x} . \hat{K}$ represents the exchange operator that arises from the antisymmetry of the wave function.

$$
\hat{K}_{i}\left(\mathbf{x}_{1}\right) \chi_{i}\left(\mathbf{x}_{1}\right)=\left[\int \frac{\left|\chi_{j}^{*}\left(\mathbf{x}_{2}\right) \chi_{i}\left(\mathbf{x}_{2}\right)\right|^{2}}{\mathbf{r}_{i j}} \mathrm{~d} \mathbf{x}_{2}\right] \chi_{\mathbf{j}}\left(\mathbf{x}_{1}\right) .
$$


Furthermore, it needs to be mentioned that the HF method does not allow self-interaction to occur since in a non-zero Coulomb interaction in the case of $\mathrm{i}=\mathrm{j}$ is canceled out by the exchange term. This self interaction problem, that is fully taken care of in HF, becomes however, a big issue in density functional theory, discussed in chapter 2.3.].

Equation 2.18 shows that due to the Fock operator the HF potential depends on all occupied orbitals. Therefore it depicts a non linear problem, with so called "pseudo eigenvalue" equations that need to be solved numerically, which is done within the selfconsistent-field approach (SCF). By means of a guessed set of spin orbitals, leading to an effective HF potential, the HF equations can be solved. This results in a new set of orbitals that can be used in the next iteration until the obtained orbitals yield an effective potential that is not changing within a certain threshold, thus becomes self-consistent. Since the numeric solution of the HF integro-differential equations is even now, still very challenging even further simplifications have to be made. Roothaan and Hall suggested to expand the orbitals in terms of $N$ basis functions $\theta$ with corresponding coefficients $c$. This Ansatz became known as the linear combination of atomic orbitals method (LCAO) by Roothaan-Hall [11, [20].

$$
\phi_{i}=\sum_{a}^{N} c_{a} \theta_{a} .
$$

An infinite set of basis functions would lead to an exact description of the orbitals. Unfortunately, in practice, the basis set has to be finite leading to a approximation of the orbitals. Further information about basis sets can be found in sections 2.3 .4 and 2.4.7. Applying the LCAO method to the HF equations leads to the Hartree-FockRoothaan-Hall matrix eigenvalue equation

$$
F C=\epsilon S C
$$

with $F$ as the Fock-matrix, $C$ a matrix containing the coefficients $c$ of the corresponding molecular orbital, $\epsilon$ a diagonal matrix containing the orbital energies and the overlap matrix $S$ which is needed since the basis functions, centered at different atoms, are nonorthogonal.

This procedure was initially developed for the common situation of closed-shell systems. 
Within the so called "restricted Hartree-Fock method" (RHF) the orbital expansion for these molecules is usually realized by assuming that two spins ( $\alpha$ and $\beta$ ) share one spatial orbital leading to the Hartree-Fock-Rothaan-Hall equation 2.2.3, as explained above. In the case of systems that exhibit unpaired electrons the common method of choice within the HF formalism is the "unrestricted Hartree-Fock method" (UHF). Unlike RHF where one spatial orbital is occupied by two spins, UHF uses different orbitals for the $\alpha$ and $\beta$ spin leading to two coupled Hartree-Fock-Rothaan-Hall equations that are known as Berthier-Pople-Nesbet equations ${ }^{[121]}$. The spatial separation of the $\alpha$ and $\beta$ spin leads to different orbital energies and hence to the UHF orbitals not being eigenfunctions of the total spin operator $\hat{S}$. This however leads to a spin contamination of the UHF orbitals. Nowadays, Hartree-Fock is a method of mostly conceptual value. The error arising from a lack of the correlation energy which has been initially defined by Löwdin as the difference between the exact non relativistic energy $E_{0}$ and the HF energy $E_{\mathrm{HF}}{ }^{[121]}$,

$$
E_{\text {corr }}=E_{0}-E_{\mathrm{HF}},
$$

is essential in order to make accurate predictions about quantum chemical systems. Thus, many other methods that are based on HF have been developed (for detailed description see: Ref. ${ }^{[18]}$ ). Post Hartree-Fock methods like coupled-cluster, configuration interaction or Møller-Plesset perturbation theory offer more accurate ways in describing the properties of many particle systems by additionally considering excited determinants on top of the ground state Slater-determinat. Nevertheless those are usually not the methods of choice when dealing with large systems.

\subsection{Density Functional Theory}

While all the methods mentioned so far are based on the wave function of a system, the approach density functional theory (DFT) takes is relying on the electron density. The main advantage of DFT is the computational affordability of systems larger than just a few atoms by maintaining sufficient accuracy, which made it the dominant method for quantum chemical simulations of periodic systems and large molecules. 
The earliest form of DFT goes back to the late 1920's when Thomas ${ }^{[122]}$ and Fermi ${ }^{[ए 23]}$ were the first to use the electron density to describe the total energy of a system. In 1964 Hohenberg and Kohn improved their idea by postulating two theorems which laid the foundations for modern DFT [04]. The first theorem proves that a certain electron density exactly determines a unique external potential and contains all information about $\mathbf{r}_{A}, Z_{A}$ and $N$. Therefore the energy $E$ of an $n$-particle system is a functional of the ground-state electron density $\rho$ and can be described by $\rho$ in an external potential $V_{\mathrm{s}}$.

The second theorem states that the variational principle can be applied to every trial density function $\rho_{\mathrm{T}}$, normalized to the number of electrons $N$, while the energy will always be greater or equal to the total ground state energy $E_{0}$,

$$
E_{0} \leq E\left[\rho_{\mathrm{T}}\right]
$$

\subsubsection{Kohn-Sham Equations}

Shortly after the two fundamental theorems where established, Kohn and Sham introduced a method to approximately solve the electronic time independent, non relativistic Schrödinger equation of a system of interacting electrons based on DFT calculations ${ }^{[125]}$. Kohn and Sham (KS) method suggested to map the ground state electron density of a system of interacting electrons onto a reference system of $N$ non-interacting electrons in an external potential $V_{\mathrm{s}}{ }^{[12 \mathrm{~T}]}$. The Hamiltonian of the whole non-interacting system $\mathrm{s}$ can be written as the sum over the effective one-electron Hamiltonians $\hat{h}_{i}^{\mathrm{KS}}$

$$
\hat{H}_{\mathrm{s}}=\sum_{i=1}^{n}\left(-\frac{1}{2} \Delta_{i}+V_{\mathrm{s}}\left(\mathbf{r}_{i}\right)\right)=\sum_{i=1}^{n} \hat{h}_{i}^{\mathrm{KS}} .
$$

Applying the one-electron Hamiltonians on the Kohn-Sham orbitals $\phi_{i}^{\mathrm{KS}}$ to obtain the orbital energies $\epsilon_{i}^{\mathrm{KS}}$ can be expressed as

$$
\hat{h}_{i}^{\mathrm{KS}}\left|\phi_{i}^{\mathrm{KS}}\right\rangle=\epsilon_{i}^{\mathrm{KS}}\left|\phi_{i}^{\mathrm{KS}}\right\rangle
$$

The external potential has to be chosen such that the density resulting from the KohnSham orbitals corresponds to the density of the real interacting system. 
Utilizing this Ansatz, the energy of the system as a function of the electron density can be written as

$$
E[\rho]=\int \rho(\mathbf{r}) V_{\mathrm{s}}(\mathbf{r}) \mathrm{d} \mathbf{r}+T_{\mathrm{s}}[\rho]+\frac{1}{2} \iint \frac{\rho\left(\mathbf{r}_{1}\right) \rho\left(\mathbf{r}_{2}\right)}{\left|\mathbf{r}_{12}\right|} \mathrm{d} \mathbf{r}_{1} \mathrm{~d} \mathbf{r}_{2}+\Delta T[\rho]+\Delta V_{\mathrm{ee}}[\rho],
$$

where $\Delta T[\rho]$ is the difference between the kinetic energy of the real interacting system and the kinetic energy of the non-interacting system $\mathrm{s}$

$$
\Delta T[\rho]=T[\rho]-T_{\mathrm{s}}[\rho]
$$

For $\Delta V_{\text {ee }}[\rho]$ accounts

$$
\Delta V_{\mathrm{ee}}[\rho]=V_{\mathrm{ee}}[\rho]-\frac{1}{2} \iint \frac{\rho\left(\mathbf{r}_{1}\right) \rho\left(\mathbf{r}_{2}\right)}{\left|\mathbf{r}_{12}\right|} \mathrm{d} \mathbf{r}_{1} \mathrm{~d} \mathbf{r}_{2}
$$

Both $\Delta T[\rho]$ and $\Delta V_{\mathrm{ee}}[\rho]$ are the only unknown terms in eq. 2.27 and are therefore summarized in the exchange-correlation functional $E_{\mathrm{XC}}[\rho]$ defined as

$$
E_{\mathrm{XC}}[\rho]=\Delta V_{\mathrm{ee}}[\rho]+\Delta T[\rho]
$$

In theory this method should yield an exact solution. However, since the exchangecorrelation functional is only known for the free electron gas, it needs to be approximated for any other system.

\subsubsection{Exchange and Correlation Functionals}

The development of new exchange-correlation functionals is a very active field of research. Up until now many different approaches have been proposed. The most common approximations, LDA, GGA and hybrid functionals, will be briefly discussed in the following subsections. 


\section{Local Density Approximation (LDA)}

A first approach towards the determination of the exchange-correlation functional was done by introducing the local density approach. The central idea behind the functional is based on the homogeneous electron gas. This model assumes the inhomogeneous electron density of a system to be locally homogeneous, which might work well for metals but fails to describe the rapid change in electron density present in a molecule. The exchange-correlation energy of the LDA approximation can be written as

$$
E_{\mathrm{XC}}^{\mathrm{LDA}}[\rho]=\int \rho(\mathbf{r}) \epsilon_{\mathrm{XC}}[\rho] \mathrm{d} \mathbf{r}
$$

with the exchange-correlation potential $\epsilon_{\mathrm{XC}}[\rho]$ of a homogeneous electron gas as the sum of the correlation potential $\epsilon_{\mathrm{C}}[\rho]$ and the exchange potential $\epsilon_{\mathrm{X}}[\rho]$. While the exchange part was originally derived by Bloch and Dirac ${ }^{[126]}$, no explicit description for the correlation part is known. Two of the most famous correlation contributions to $\epsilon_{\mathrm{XC}}$ were derived by Vosko, Wilk and Nusair using quantum Monte Carlo calculations ${ }^{[127]}$.

\section{Generalized Gradient Approximation (GGA)}

In order to include the inhomogeneity of the electron density distribution in molecules the generalized gradient approximation was developed. The GGA functional additionally considers the density gradient $\nabla \rho(\mathbf{r})$. Thus, the exchange-correlation energy can by defined by

$$
E_{\mathrm{XC}}^{\mathrm{GGA}}[\rho]=\int \rho(\mathbf{r}) \epsilon_{\mathrm{XC}}[\rho, \nabla \rho] \mathrm{d} \mathbf{r} .
$$

Some examples of GGA functionals are the frequently used Perdew-Wang-91 functional $^{[128]}$ and the Perdew-Burke-Ernzerhof functional (PBE) ${ }^{[129,1330]}$. The latter has been used extensively throughout this thesis. Further improvement has been achieved trough the use of meta-GGA functionals, which include higher order derivatives of the electron density. 


\section{Hybrid Functionals}

In contrast to GGA and LDA, hybrid functionals mix the exact exchange term determined by using the Kohn-Sham orbitals and applying the Hartree-Fock expression with the exchange and correlation part obtained from LDA/GGA. Therefore hybrid functionals can be generally written as

$$
E_{\mathrm{XC}}^{\mathrm{hybrid}}=\alpha E_{\mathrm{X}}^{\mathrm{HF}}+E_{\mathrm{C}}+(1-\alpha) E_{\mathrm{X}}^{\mathrm{LDA} / \mathrm{GGA}}
$$

where $E_{\mathrm{X}}^{\mathrm{HF}}$ the exact exchange energy, $\alpha$ is the fraction the exact exchange energy that is mixed in, $E_{\mathrm{X}}^{\mathrm{LDA} / \mathrm{GGA}}$ the LDA/GGA exchange energy and $E_{\mathrm{C}}$ the correlation energy. B3LYP, developed by Becke, Lee, Yang and Parr ${ }^{[131,[132]}$ represents one of the most commonly used hybrid functionals. The development of hybrid functionals often lead to an improvement of the accurate description and estimation of many properties like band gaps, bond lengths and vibration frequencies.

\subsubsection{Dispersion Corrections}

Dispersion interactions are present in all systems. Due to the local nature of the previously discussed LDA and GGA functionals long-range interactions are not taken into account sufficiently, which leads to the necessity of a new method in particular for extended systems. One method to include long-range interactions supplementary to DFT calculations, is the empirical DFT-D approach developed by Grimme. In the framework of this thesis DFT-D3 ${ }^{[133]}$ corrections have been employed when considered appropriate. The dispersion energy $E_{\text {disp }}$ can be simply added to the DFT energy $E_{\mathrm{DFT}}$. Thus, the dispersion corrected total energy $E_{\mathrm{DFT}-\mathrm{D}}$ is given by

$$
E_{\mathrm{DFT}-\mathrm{D}}=E_{\mathrm{DFT}}+E_{\mathrm{disp}}
$$

The empirical dispersion correction is defined as a sum of the two and three body terms $E_{2}$ and $E_{3}$ respectively,

$$
E_{\text {disp }}=E_{2}+E_{3} \text {. }
$$


The two body term can be written as

$$
E_{2}=\sum_{A B} \sum_{n=6,8} s_{n} \frac{C_{n}^{A B}}{r_{A B}^{n}} f_{\mathrm{dmp}, \mathrm{n}}\left(r_{A B}\right)
$$

where $C_{n}^{A B}$ denotes the dispersion coefficient of atom pair $A B$ up to the $\mathrm{n}^{\text {th }}$-order (here 8), $r_{A B}$ is the interatomic distance, $s_{6} / s_{8}$ is a scaling factor depending on the employed functional and $f_{\mathrm{dmp}}$ is the damping function. The three body term $E^{3}$ usually makes up $5-10 \%$ of $E_{\text {disp }}$ and is denoted by,

$$
E_{3}=\sum_{A B C} f_{\mathrm{dmp}, 3}\left(\bar{r}_{A B C}\right) E_{A B C}
$$

where $\bar{r}_{A B C}$ are the geometrically averaged radii and $E_{A B C}$ the nonadditive dispersion term derived from the third-order perturbation theory for the three atoms $\mathrm{A}, \mathrm{B}$ and C. A damping function $f_{\mathrm{dmp}}$ has to be applied to avoid unphysical behavior for small interatomic distances. The commonly used "zero damping" $f_{\text {dmp-zero }}$ has the form ${ }^{[\amalg 33]}$

$$
f_{\mathrm{dmp}-\mathrm{zero}}\left(r_{A B}\right)=\frac{s_{n}}{1+6\left(r_{A B} /\left(s_{r, n} R_{0}^{A B}\right)\right)^{-\alpha_{n}}},
$$

where $R_{0}^{A B}$ are the cutoff radii and $s_{r, n}$ its nth-order dependent scaling factor and $\alpha_{6}$ and $\alpha_{8}$ are fixed to the values 14 and 16 respectively. Whereas the Becke-Johnson damping $f_{\text {dmp-bj }}$ can be written as ${ }^{[134]}$

$$
f_{\mathrm{dmp}-\mathrm{bj}}\left(r_{A B}\right)=\frac{s_{n} r_{A B}^{n}}{r_{A B}^{n}+\left(a_{1} R_{0}^{A B}+a_{2}\right)^{n}},
$$

with $a_{1}$ and $a_{2}$ as functional fitted parameters.

\subsubsection{Basis Sets and Effective Core Potentials}

Section 2.2 already introduced the idea of expanding the orbitals by a finite set of basis functions. For a given number of basis functions some choices lead to a smaller error than others. A good choice are atom centered Slater Type Orbitals (STO), as these 
functions render the appropriate exponential decay of the wave function with respect of the distance from the nuclei. The general form of an STO $\phi^{\mathrm{STO}}$ is denoted by

$$
\phi^{\mathrm{STO}}=N Y_{l, m} r^{n-1} e^{-\zeta_{S} r}
$$

$N$ represents a normalizing constant, $r^{n-1} e^{-\zeta_{S} r}$ the radial part of the STO with $r$ as the distance of the electron from the nucleus and $\zeta_{S}$ as the orbital exponent. $Y_{l, m}$ is the angular part of the STO, where it is common to use spherical harmonics ${ }^{[135]}$. However, STOs are computationally difficult and therefore in practice usually approximated by a linear combination of contracted Gaussian type orbitals (GTO)

$$
\phi^{\mathrm{STO}}=\sum_{i} a_{i} \phi_{i}^{\mathrm{GTO}}
$$

with $a$ as the contraction coefficient and the Gaussian type orbitals $\phi^{\mathrm{GTO}}$ of the general form

$$
\phi^{\mathrm{GTO}}=N x^{l} y^{m} z^{n} e^{-\zeta_{G} r^{2}} .
$$

Here $\zeta_{G}$ represents the orbital exponent and $l, m$ and $n$ are used to classify the GTOs with $L=l+m+n$, where $L=0$ represents $s$-type functions, $L=1 p$-type functions etc.

In order to obtain a flexible basis set that resembles the real electronic structure as close as possible, one orbital can be described by several basis functions exhibiting different orbital coefficients. The method of using two basis functions to describe one atomic orbital is called "Double-Zeta basis set" (DZ), using three basis functions is called "Triple-Zeta basis set" (TZ) etc. Furthermore polarization functions and diffuse functions can be added to achieve a higher accuracy, which may be required in specific cases such as in the description of anions. A disadvantage arising from the use of atom centered basis sets is the so called basis set superposition error (BSSE). The error is caused by two or more interacting molecules being close enough to use the basis functions localized at the other molecule leading to an over stabilization of the dimer or complex due to more basis functions being available for the wavefunction expansion. The basis set superposition error is usually accounted for by applying the counterpoise correction $(\mathrm{CP})^{[1035]}$. A 
popular choice of basis functions for solid state systems are plane waves which will be further described in 2.4.].

To further minimize the computational effort but while retaining the accuracy of the calculations, Hellmann suggested to use pseudopotentials to account for the influence of the core electrons and the nucleus on the valence electrons ${ }^{[136]}$. This idea is based on the assumption that the core orbitals experience very small changes during chemical processes, which can be neglected. The so called "effective core potentials" (ECP) are obtained through numerical HF or Dirac-HF calculations and have the big advantage that relativistic effects can partially be included without additional computational effort.

As a non-wave function based method that represents an improvement of the HF method, DFT has many advantages where the most important one is certainly the reduced computational effort. Nevertheless DFT reveals several disadvantages. The exchangecorrelation functionals are only empirical approximations therefore the method can not be systematically improved. Furthermore it is not possible to predict the size of the error. A large disadvantage comes in to play when dealing with systems where excited states are central for the chemical behavior and properties since DFT is a method to calculate the electronic ground state of a system.

To obtain further information from the DFT, HF, MP2 etc. results about the regarded system many different tools have been developed. E.g. tools to calculate the charge of the individual atoms in the system such as natural population analysis (NPA) ${ }^{[137]}$, Mulliken population analyses (MPA) ${ }^{[138,[39]}$ and Bader charge analysis (BCA) [140-[142]. Within this thesis the latter method has been chosen for the description of atomic charges. The Bader formalism divides the charge between atoms by introducing planes wherever the charge density reaches a minimum, enclosing a particular volume around each atom. The Volume can in a next step be used to calculate the charge within, thus the charge of each individual atom. A tool that has particularly been used for the project work discussed in subsection 7.2 is the nudged elastic band (NEB) ${ }^{[143,144]}$. Nudged elastic band offers the opportunity to calculate the minimum energy path for the rearrangement of atoms between two local minimum structures that are provided at the start of the calculation. This is realized by optimizing a certain amount of "pictures" of the investigated system 
along the energy path which are connected to the previous and next picture by spring forces in order to keep each image from sliding down the energy landscape. Another central tool is constituted by the energy decomposition analysis (EDA), a method developed by Ziegler and Rauk ${ }^{[145]}$. The bond energy of a system consisting of two fragments $\mathrm{A}$ and $\mathrm{B}$ is defined as follows,

$$
E_{\text {bond }}=\Delta E_{\text {prep }}+\Delta E_{\text {int }}
$$

where $\Delta E_{\text {prep }}$ is the preparation energy that is needed in order to structurally transform fragment $\mathrm{A}$ and $\mathrm{B}$ in their ground state to their states in the system $\mathrm{AB} . \Delta E_{\mathrm{int}}$, however, is the interaction energy between the two fragments of the regarded system. Utilizing the Hartree product of the wave functions of fragment $\mathrm{A}$ and $\mathrm{B}$ in their individual excited states in the system allows to separate the interaction energy into physically understandable components such as the Pauli repulsion, the electrostatic interaction between the fragments and the orbital interaction.

\subsection{Quantum Theory of Solids}

The theoretical background that has been discussed so far is dealing with the quantum mechanical description of finite systems. Non finite systems, such as crystalline solids are still an impressive challenge to theoretical chemistry but can be addressed in a computational quantum mechanical manner by introducing certain simplifications. The following sections give a brief introduction about the main aspects of solid state theory and the computation of this material class, that are needed in order to understand the content of this thesis.

It is a well established concept that crystals are infinite systems that are defined by a periodic lattice, the so called "Bravais lattice". The Bravais lattice defines the structure of a system whereas the specific location of the atoms within the lattice is given by the basis ${ }^{[146]}$. Combining both yields the so called "unit cell" which completely determines the structure of the smallest possible periodic subunit the regarded crystal is made of. Thus, the whole system can be built up by a translation of the unit cell along the 
translational vector $\mathbf{T}$ where $\mathbf{a}_{1}, \mathbf{a}_{2}$ and $\mathbf{a}_{3}$ are the non-collinear basis vectors of the unit cell in real space and $n_{1}, n_{2}$ and $n_{3}$ are integers ${ }^{[[9]}$.

$$
\mathbf{T}=n_{1} \mathbf{a}_{1}+n_{2} \mathbf{a}_{2}+n_{3} \mathbf{a}_{3}
$$

A mathematically handy way to investigate crystalline solids is the introduction of the reciprocal space. The crystal can be described in reciprocal space by using the reciprocal lattice vector $\mathbf{K}$ of the form

$$
\mathbf{K}=m_{1} \mathbf{b}_{1}+m_{2} \mathbf{b}_{2}+m_{3} \mathbf{b}_{3},
$$

where $m_{1}, m_{2}$ and $m_{3}$ are integers and $\mathbf{b}_{1}, \mathbf{b}_{2}$ and $\mathbf{b}_{3}$ are the reciprocal basis vectors. These vectors can be expressed in terms of the real space vectors as

$$
\begin{aligned}
& \mathbf{b}_{1}=\frac{2 \pi}{V} \mathbf{a}_{2} \times \mathbf{a}_{3}, \\
& \mathbf{b}_{2}=\frac{2 \pi}{V} \mathbf{a}_{3} \times \mathbf{a}_{1}, \\
& \mathbf{b}_{3}=\frac{2 \pi}{V} \mathbf{a}_{1} \times \mathbf{a}_{2},
\end{aligned}
$$

where $V$ is the volume of the unit cell in real space ${ }^{[135,1477}$. The symmetry of the reciprocal space reflects the symmetry of the real space, whereas the lattice vectors of real and reciprocal space have an inverse relationship. This results in long distances in real space being represented by short distances in reciprocal space and vice versa.

The infinity of crystalline systems depicts a huge computational obstacle, nevertheless through utilizing the translational symmetry of those systems this problem can be addressed. In 1929 Bloch published his theorem that laid the foundation of the modern computational description and treatment of solid state materials. Bloch considered the electrons to independently move in a static periodic potential, which is due to the translational symmetry of the system. In order to obey the periodicity of the potential i.e. 
the periodic Hamilton operator, he stated that the energy eigenfunction for one particle in a periodical potential can be written as ${ }^{[148]}$

$$
\psi(\mathbf{k}, \mathbf{r})=e^{i \mathbf{k r}} u(\mathbf{r}) .
$$

The so called "Bloch wave" consists of a function with the periodicity of the potential $u(\mathbf{r}+\mathbf{T})=u(\mathbf{r})$, where $\mathbf{r}$ is a real space vector and $\mathbf{T}$ the translational vector in real space, multiplied with the plane wave function $e^{i \mathbf{k} \mathbf{T}}$ with $\mathbf{k}$ as a vector in reciprocal space $^{[149]}$.

Due to the periodicity of the regarded system the wave function is required to be identical at the boundaries of the unit cell, which is given by

$$
\psi(\mathbf{k}, \mathbf{r})=e^{i \mathbf{k r}} u(\mathbf{r})=\psi(\mathbf{k}, \mathbf{r}+\mathbf{T})=e^{i \mathbf{k T}} e^{i \mathbf{k r}} u(\mathbf{r}+\mathbf{T})
$$

Since $u$ fulfills the translational symmetry, it is required that $e^{i \mathbf{k T}}=1$ which is according to Euler's law fulfilled when $\mathbf{k T}$ is a multiple of $2 \pi$. Hence, specific boundary conditions for $\mathbf{k}$ arise.

$$
-\frac{\pi}{|\mathbf{T}|} \leq|\mathbf{k}| \leq \frac{\pi}{|\mathbf{T}|}
$$

These values for $\mathbf{k}$ define the range where all unique wave functions are found and further define the volume of the smallest possible primitive cell in reciprocal space, the so called "first Brillouin zone". Up until now, we have considered a crystal to consist of infinitely translated primitive unit cells. A mathematically more handy way is to construct the "Wigner-Seitz cell" which always corresponds to the smallest possible unit cell. The Brillouin zone is constructed in reciprocal space analogous to the Wigner-Seitz cell in real space. Fig. 2.1 shows the construction scheme of the smallest primitive cell in real space.

The wavevector $\mathbf{k}$ is in principle continuous and can adopt every possible value within the first Brillouin zone. However, to realize the description of such a system only a finite number of points can be taken into account. In practice, a three dimensional $k$-mesh is applied to calculate discrete points within the first Brillouin zone. One of the best known methods for the choice of an appropriate $k$-mesh was developed by Monkhorst 


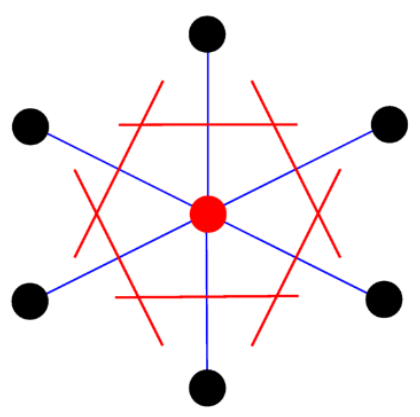

Figure 2.1: Two-dimensional construction scheme of the Wigner-Seitz cell to obtain the smallest possible unit cell, the "primitive cell" [150]. The blue lines represent the connection between one lattice point and symmetry equivalent lattice points, which are often the nearest neighbors. The red lines indicate perpendicular planes that cut the connecting lines in half and furthermore enclose the volume of the Wigner-Seitz cell.

and Pack ${ }^{[\square]}$.

Furthermore it has to be mentioned that today's quantum chemical program packages model a crystal by applying periodic boundary conditions known as "Born-von Karman boundary conditions" [52], where the number of unit cell replicas in each direction of the lattice vectors is determined by the number of $k$-points chosen for defining the $k$-grid along every direction.

\subsubsection{Plane Wave Basis Set}

Program packages for the quantum mechanical description of extended systems usually make use of DFT. Section 2.3.4 already introduced the concept of a basis set for the determination of the functional form of the Kohn-Sham orbitals. It was mentioned that the most common approach is a linear combination of a set of appropriate basis functions. In the case of a solid state system a well suited choice of basis functions is offered by plane waves as introduced in Equation 2.47. Thus, the Kohn-Sham orbitals can be expressed as follows

$$
\phi^{\mathrm{KS}}(\mathbf{r}, \mathbf{k})=\sum_{\mathbf{K}} c_{n}(\mathbf{k}, \mathbf{K}) e^{i(\mathbf{k}+\mathbf{K}) \mathbf{r}}
$$


where $\mathbf{K}$ is the reciprocal lattice vector as defined in equation 2.4 .5 and $c_{n}(\mathbf{k}, \mathbf{K})$ are coefficients that have to be determined numerically or analytically. Furthermore, the basis set has to be finite, hence only plane waves up to a certain cut-off energy $E_{\text {cut }}$ are included in the expansion ${ }^{[149]}$

$$
E_{\text {cut }} \leq \frac{1}{2}|\mathbf{k}+\mathbf{K}|^{2}
$$

The use of plane waves offers several advantages. One very obvious reason is the simplicity of plane waves that reduces computational demand. Moreover plane waves are eigenfunctions of the homogeneous electron gas. Due to this reason, they naturally fit to the crystal's boundary conditions and reproduce the delocalization of the lattice electrons within a crystal. Another aspect that needs to be mentioned, is that a plane wave basis set is not atom centered and therefore does not suffer from a basis set superposition error.

Despite all those advantages a significant problem arises. Due to the rapid oscillation of the radial part of the wave function in the core region of heavier atoms, a large amount of plane waves would be required for a proper description of this region. In order to circumvent this problem pseudopotentials (PP) can be applied, which have been developed to describe the interaction between the valence electrons and the potential created by the core electrons and the nucleus ${ }^{[149]}$. Solely the valence electrons are treated explicitly. A divers array of pseudopotentials has been developed ${ }^{[153,154]}$. One of the most common method that uses pseudopotentials is the projector augmented wave method (PAW) [15, , The main idea behind PAW is that at a certain distance from the nucleus the wave function of a system is smooth, so that it can be appropriately described by plane waves. This method approaches the problem of the rapid oscillations close to the nucleus by transforming the wave functions into smooth wave functions from which all electron properties can be calculated. In contrast to other pseudopotential methods, PAW formally retains all core electrons ${ }^{[135]}$.

\subsubsection{Band Structure and Density of States}

In order to understand the chemical or physical behavior of any system it is necessary to study its electronic structure. From the studies of individual atoms it is known that their 
electrons can occupy different atomic orbitals and therefore take discrete energy levels. Combining several atoms leads to an overlap of the atomic orbitals causing the formation of molecular orbitals with more energy states per energy interval being available for the electrons to be occupied. Thinking of even larger systems, like bulk systems with an amount of atoms close to infinity, leads to an even finer splitting of the states. The states can be regarded as continuous. A plot of those energy eigenvalues within the first Brillouin zone and in dependence of the reciprocal space vector $\mathbf{k}$ is called the "band structure". The band structure and a potential band gap help to classify materials as metals, semiconductors and insulators and moreover to understand properties like electron transport.

Another helpful tool to investigate the electronic structure of a system is the density of states (DOS). The DOS is defined as the number of states per energy interval that are available at each level for the electrons to be occupied. Following this definition, the DOS can be obtained by calculating the one electron levels within a small energy interval dE. It holds, that

$$
\operatorname{DOS}(\mathrm{E}) \sim \frac{\mathrm{dn}}{\mathrm{dE}}
$$

where $\mathrm{dn}$ is the number of states available per interval. The DOS is therefore nothing else but the inverse slope of the regarded band. What makes this method especially interesting is the possibility to project the DOS on to e.g. the spherical harmonics, orbitals, atoms or orbital contributions of individual atoms (PLDOS), to further analyze the properties of the investigated systems.

A projection method that has been intensively used throughout this thesis, is the angular momentum projected density of states (PDOS) which was utilized to analyze the global electronic structure e.g. the superatomic nature of clusters and cluster assembled materials. The projection is realized by projecting the Kohn-Sham orbitals $\phi^{\mathrm{KS}}$ onto the spherical harmonics $Y_{l, m}$. The projections is made within a sphere of the radius $R_{0}$ enclosing the investigated cluster core. The sphere has to be chosen such that the center 
of mass is the center for the projection. The coefficients (angular momentum weights) of each Kohn-Sham orbital are given as $[157,[158]$

$$
c_{i, l}\left(R_{0}\right)=\sum_{m} \int_{0}^{R_{0}} \mathbf{r}^{2} \mathrm{~d} \mathbf{r}\left|\mathrm{f}_{\mathrm{i}, 1, \mathrm{~m}}(\mathbf{r})\right|^{2},
$$

where $f_{i l l, m}$ is defined as

$$
f_{i, l, m}(\mathbf{r})=\int \mathrm{d} \hat{\mathrm{r}} \mathrm{Y}_{1, \mathrm{~m}}^{*}(\hat{\mathrm{r}}) \phi^{\mathrm{KS}}(\mathbf{r})
$$

with $i$ the index of the Kohn-Sham orbital, $l$ the angular momentum number and $m$ the magnetic number.

\subsubsection{Computational Details}

Since the investigations presented in this thesis are purely of theoretical nature it is necessary to mention the computational details, in particular the programs used. Specific details that apply for the computation of the different systems will be given in each of the results chapters. In general it can be stated that all calculations presented are based on Kohn-Sham density functional theory. One of the central programs used for the analysis is Vienna Ab initio Simulation Package (VASP) ${ }^{[159-163]}$. VASP is a program package with periodic boundary conditions which makes it necessary to include sufficient vacuum in order to avoid unwanted interactions between neighboring particles when dealing with individual clusters. In addition in the case of clusters a $1 \times 1 \times 1 \Gamma$-centered MonkhorstPack ${ }^{[151]} k$-mesh was applied in all cases. Furthermore the scalar relativistic projector augmented wave approach ${ }^{[155,156]}$ has been utilized unless stated otherwise.

For all calculations conducted using the computational chemistry program Turbomole 7.0.1 $\left[{ }^{[64]}\right.$ the Multipole Accelerated Resolution of Identity approximation has been used. Moreover, the program package Amsterdam Density Functional 2014 (ADF) ${ }^{[65, \pi 66]}$ has been utilized in order to calculate specific spin states and to conduct energy decomposition analysis. In addition, the freely available Bader analysis software by Henkelman et al. ${ }^{[140-[42]}$ was used to analyze the Bader charges of certain systems. 


\section{Tunability of Superatoms}

\subsection{Introduction}

The $\mathrm{Au}_{13}\left[\mathrm{RS}(\mathrm{AuSR})_{2}\right]_{6}$ cluster has already been introduced as one of the smallest thiolate protected superatomic gold clusters revealing high thermodynamic stability. In

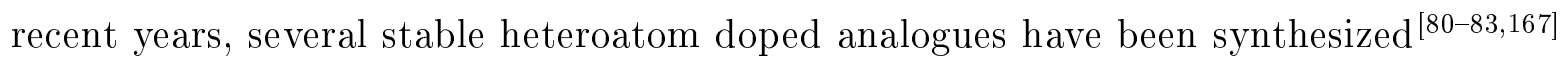

and analyzed, both experimentally ${ }^{[84-87]}$ and theoretically ${ }^{[[2,[88,001,168]}$, which has raised the question in how far a small chemical impurity can influence the overall behavior of those clusters.

The following part of this thesis addresses the change of the electronic structure of singly heteroatom doped clusters, to show the effect of doping on the superatomic structure depending on both the valency and position of the dopant. Therefore, the applicability of the classically used superatomic model on the differently doped clusters is critically evaluated, and related to the degree of structural distortion and electronic inhomogeneity. Furthermore, such an analysis gives an idea about the tunability of the electronic structure through doping. This knowledge is very valuable when it comes to possible stoichiometric cluster assembled materials. Dopants can be used to fine tune the electronic properties of clusters obtain stable functional materials exhibiting desired properties to in a next step.

For the investigations conducted in the framework of this thesis the precious metals copper $(\mathrm{Cu})$, silver $(\mathrm{Ag})$, palladium $(\mathrm{Pd})$ and platinum $(\mathrm{Pt})$ have been chosen as dopants since these individual atom doped clusters have already been synthesized. According to the superatom model these clusters should in theory be electron acceptors. In order to cover the electron donor side, the $p$-block metals aluminum (Al), gallium (Ga), indium $(\mathrm{In})$, germanium $(\mathrm{Ge})$ and tin $(\mathrm{Sn})$ have additionally been chosen as dopants and the 
corresponding doped gold clusters have been theoretically investigated.

The work presented in this chapter has partially been published in Ref. ${ }^{[89]}$.

\subsection{Methods}

The following results are all obtained using the program package VASP, whereby the generalized gradient approximation PBE ${ }^{[129,130]}$ was chosen as DFT functional. Single point- and structure optimization calculations have been performed spin unpolarized with additional dipole and quadrupole corrections. Structure optimizations have been performed without symmetry restrictions using the X-ray structure of crystalline $\mathrm{Au}_{13}\left[\mathrm{RS}(\mathrm{AuSR})_{2}\right]_{6}{ }^{[\mathrm{[T}]}$ as the initial input structure for all differently doped clusters. Moreover all calculated structures have been simplified by replacing the phenyl groups of the ligands of the cluster with methyl groups, since this does not change the overall picture of the electronic structure at the cluster center but reduces the computational effort. As energy cut-off of the plane waves, $414 \mathrm{eV}$ was chosen. To ensure high accuracy the energy convergence criterion was set to $10^{-6} \mathrm{eV}$ and the ionic relaxation was stopped when all forces dropped below $0.01 \AA / \mathrm{eV}$. Moreover, the Brillouin zone integration was done using Gaussian smearing with a smearing factor of 0.01 or lower, to obtain integer band occupation.

In order to investigate the superatomic electronic structure the angular momentum projected density of states ${ }^{[157,[158]}$ was calculated within a sphere of $2.9 \AA$ around the central atom of each cluster core. In addition, the local version of this projection, the PLDOS was utilized to show the individual atomic orbital contributions to the bands, whereby the cut off radius of the sphere around each atom has been chosen by default.

\subsection{Discussion}

The investigated $\mathrm{Au}_{13}\left[\mathrm{RS}(\mathrm{AuSR})_{2}\right]_{6}$ cluster can be seen visualized in Fig. 3.1$]$ where two possible doping sites are highligthed, namely the cluster core center visualized in blue and the cluster core surface visualized in gray. The staple motif exhibits another possible doping site which is neglected simply due to the fact that one of the main focuses of 
this research lies on the study of the cluster core states. As it has been mentioned in

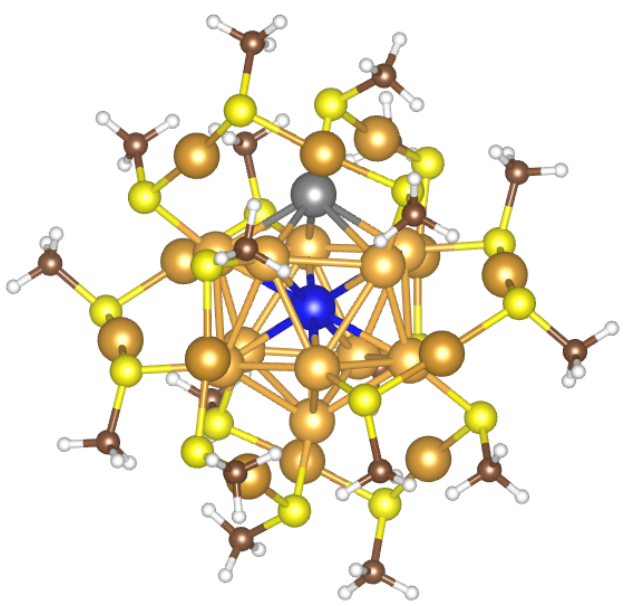

Figure 3.1: Visualization of investigated single atom doped $\mathrm{Au}_{13}\left[\mathrm{RS}(\mathrm{AuSR})_{2}\right]_{6}$ cluster, with two possible doping positions highlighted in gray and blue. Color code: Au: orange, S: yellow, C: brown and H: white.

the introduction, the gold atoms of the cluster core should in theory provide their $6 \mathrm{~s}$ electrons to the superatomic delocalized charge while each ligand localizes one of those electrons resulting in an electron count of seven superatomic electrons and therefore an $\mathrm{S}^{2} \mathrm{P}^{5}$ superatomic orbital occupation for the neutral undoped $\mathrm{Au}_{13}\left[\mathrm{RS}(\mathrm{AuSR})_{2}\right]_{6}$ cluster. An additional electron would lead to a P-shell closing with eight superatomic electrons and therefore to a highly stable cluster. Subsequently the undoped cluster and its $\mathrm{Cu}$ and $\mathrm{Ag}$ doped species should each in theory be one electron acceptors while the Pd and Pt doped species are two electron acceptors since they do not exhibit a valence $s$ electron that can contribute to the superatomic delocalized charge. Experimental studies of those clusters have shown that the preferred location of Pt and Pd within the cluster is the cluster core center, whereas Ag is preferably located at the cluster core surface $\left.{ }^{[\mathrm{B}}\right]$. Regarding $\mathrm{Cu}$ the second most stable location, the cluster core surface, is considered in this work, since experimental work and test calculation comparing relative energies have shown that the most stable position of the copper atom is within the staple motive ${ }^{[8.3]}$. In agreement with the superatom concept the $p$-block metals $\mathrm{Ga}$, In and $\mathrm{Al}$ should in theory, be one electron donors, whereas Ge and Sn are two electron donors. Due to the fact that no experimental data is available, all $p$-block atom doped clusters have been 
calculated with the necessary charge to have eight superatomic electrons $(+1$ charged in the case of $\mathrm{Ga}$, In and $\mathrm{Al}$ and +2 for $\mathrm{Ge}$ and $\mathrm{Sn}$ ). The structures have been relaxed for each dopant being located in the cluster core center and the cluster core surface. In nearly all cases the gap between the highest occupied and lowest unoccupied state (HOMO-LUMO gap) was found to be larger in the case of the cluster core center doped species, thus this position was chosen for further considerations. Whereas the $\mathrm{Al}$ doped cluster exhibits a HOMO-LUMO gap which was found to be slightly larger for the cluster core surface doped species but the total energy lower in the case of the center doped cluster core. To enable useful comparison, the dopant was chosen to be located at the cluster core center.

Fig. 32 visualizes the angular momentum projected density of states (PDOS) as discussed in subsection [2.4.2, for the differently doped clusters with different charges. The clusters chosen for this figure should, in theory, all correspond to a superatomic eight electron count and therefore a P-shell closing. The red shaded PDOS parts indicate the clearly visual superatomic states below the Fermi energy, whereas the gray shaded parts in the middle mainly correspond to the states arising form the cluster core ligand interaction, which will be supported later on. Taking a closer look at Figure 3.2 reveals similarities but also significant differences between the different projected densities of states. In agreement with the superatom model all differently doped clusters reveal a $\mathrm{P}$ like HOMO and D like LUMO state. Therefore, as expected, a P shell closing can be identified in all cases. In contrast, the splitting and intensity of the states, the HOMO-LUMO gap and the Fermi energy $E_{F}$ strongly vary with different dopants. This highlights that the superatomic structure is clearly affected by doping. Nevertheless the PDOS of the cluster cores of $\mathrm{Au}_{13}\left[\mathrm{RS}(\mathrm{AuSR})_{2}\right]_{6}^{-}, \mathrm{Au}_{12} \mathrm{Ag}\left[\mathrm{RS}(\mathrm{AuSR})_{2}\right]_{6}^{-}$and $\mathrm{Au}_{12} \mathrm{Cu}\left[\mathrm{RS}(\mathrm{AuSR})_{2}\right]_{6}^{-}$ and $\mathrm{Au}_{12} \mathrm{Pt}\left[\mathrm{RS}(\mathrm{AuSR})_{2}\right]_{6}^{2-}$ and $\mathrm{Au}_{12} \mathrm{Pd}\left[\mathrm{RS}(\mathrm{AuSR})_{2}\right]_{6}^{2-}$ still exhibit high similarity. The same applies to $\mathrm{Au}_{12} \mathrm{Al}\left[\mathrm{RS}(\mathrm{AuSR})_{2}\right]_{6}^{+}, \mathrm{Au}_{12} \mathrm{Ga}\left[\mathrm{RS}(\mathrm{AuSR})_{2}\right]_{6}^{+}$and $\mathrm{Au}_{12} \operatorname{In}\left[\mathrm{RS}(\mathrm{AuSR})_{2}\right]_{6}^{+}$ and $\mathrm{Au}_{12} \mathrm{Ge}\left[\mathrm{RS}(\mathrm{AuSR})_{2}\right]_{6}^{2+}$ and $\mathrm{Au}_{12} \mathrm{Sn}\left[\mathrm{RS}(\mathrm{AuSR})_{2}\right]_{6}^{2+}$. The similarities can be related to similar atomic valency of the corresponding group of dopants and a similar position within the cluster core. Furthermore the PDOS analysis depicts that the position of the superatomic S state varies with different dopants. In the case of the Ge and Sn doped species the S state can be found at very low energies, thus the furthest away from the 


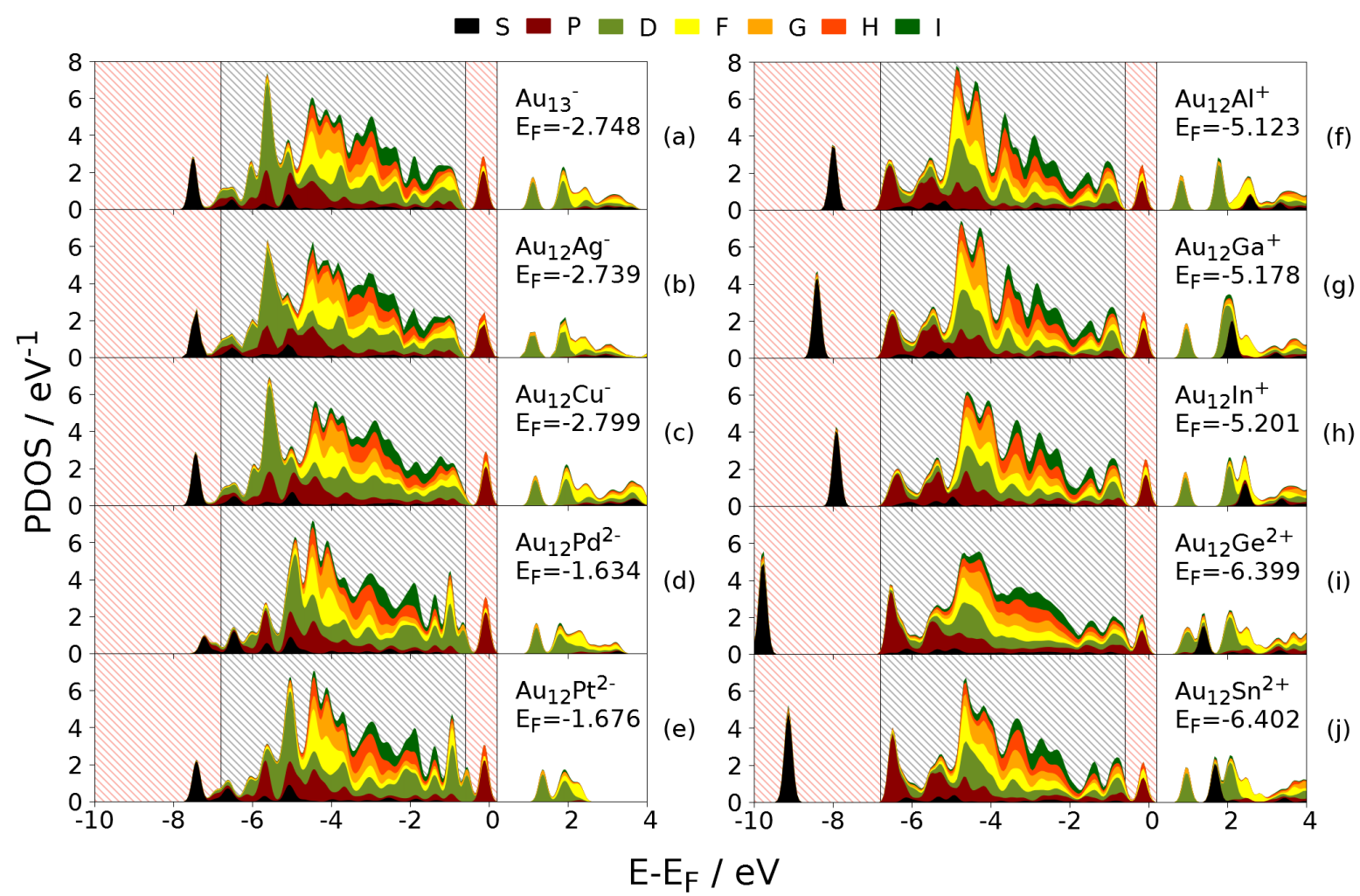

Figure 3.2: Angular momentum projected density of states of the differently doped $\mathrm{Au}_{13}\left[\mathrm{RS}(\mathrm{AuSR})_{2}\right]_{6}$ cluster cores exhibiting eight superatomic electrons, namely (a) $\mathrm{Au}_{13}^{-}$, (b) $\mathrm{Au}_{12} \mathrm{Ag}^{-}$, (c) $\mathrm{Au}_{12} \mathrm{Cu}^{-}$, (d) (b) $\mathrm{Au}_{12} \mathrm{Pd}^{2-}$, (e) $\mathrm{Au}_{12} \mathrm{Pt}^{2-}$, (f) $\mathrm{Au}_{12} \mathrm{Al}^{+}$, (g) $\mathrm{Au}_{12} \mathrm{Ga}^{+}$, (h) $\mathrm{Au}_{12} \mathrm{In}^{+}$, (i) $\mathrm{Au}_{12} \mathrm{Ge}^{2+}$, (j) $\mathrm{Au}_{12} \mathrm{Sn}^{2+}$. 
states mainly caused by the cluster core ligand interaction, while the gray shaded low energy $\mathrm{P}$ state is just an artifact of the projection. Considering the Al, Ga and In doped clusters the superatomic S state is shifted closer towards lower energies (the gray shaded zone) and even closer in the case of the undoped cluster core and the $\mathrm{Ag}$ and $\mathrm{Cu}$ doped species. Moreover, the Pt and Pd doped cores exhibit a split superatomic S state with a lower intensity compared to the other doped clusters and a ligand cluster core interaction zone which is further extended to the HOMO state. In order to explain this occurrence it has to be noted that every additional charge that is assigned to the different clusters is mainly located at the ligands which will be shown in the next sections.

The involvement of the ligands in creating those orbitals, is causing the $\mathrm{S}$ orbital effect which can be nicely seen in Figure 3 . The graphic depicts the superatomic S orbitals visualized for the undoped cluster, the $\mathrm{Pd}$ and the Ge doped cluster with the same isosurface cutoff. In the Figure is shown that in the case of the Pd doped cluster, the
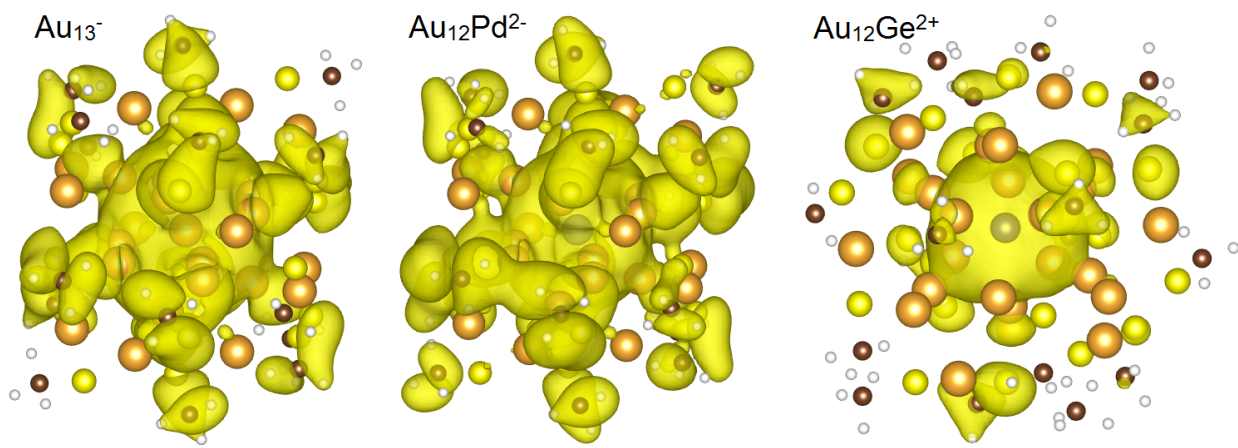

Figure 3.3: Visualization of the electron density of the superatomic $S$ orbital of the undoped and the $\mathrm{Pt}$ and Ge doped cluster highlighted with a red background shading in the PDOS figure. The isosurface cutoff was set to $1 \times 10^{-4} \mathrm{eV}$ in all cases.

$\mathrm{S}$ orbital is extended the furthest over the ligands and the least in the case of the Ge doped species, which is in good agreement with the PDOS analysis. The electrons that are mainly responsible for the occupation of the $\mathrm{S}$ orbitals are those of the central atom. Therefore the provided charge from the surrounding atoms, which is also located at the ligands, is the least in the case of the Ge and Sn doped clusters (see Fig. B.3) due to their valency, followed by the Al, Ga and In doped species. In the case of the undoped cluster and $\mathrm{Ag}$ and $\mathrm{Cu}$ doped cluster cores the additional charge that is needed for the 
S state, needs to come from the surrounding gold atoms or ligands (both are strongly interacting), whereby even more charge needs to be provided from the surroundings in the case of the Pt and Pd doped species which are lacking valence $s$ electrons.

The ligands obviously play an important role in supporting the superatomic states. Furthermore this involvement can be tuned through doping, additional charge and the position of the dopant within the cluster core. To further strengthen this statement, a calculation with the dopant located at the cluster core surface for the $\mathrm{Au}_{12} \mathrm{Pd}^{2-}$ cluster has been conducted. In Fig. 3.4 the PDOS and the PLDOS of the cluster core of the

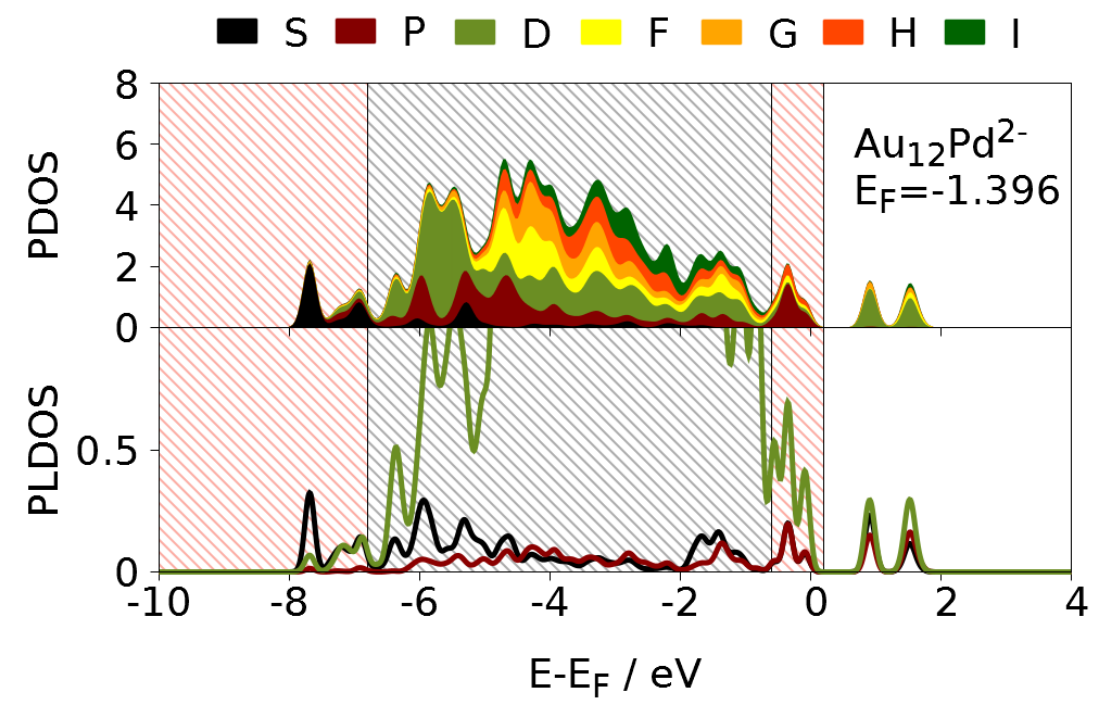

Figure 3.4: Visualization of the PDOS and PLDOS of the $\mathrm{Au}_{12} \mathrm{Pd}^{2-}$ cluster where the dopant is located at the cluster core surface.

regarded cluster are shown. The PLDOS reveals the atomic orbital contributions of the individual cluster core atoms to the bands. To ensure easier comparison with Fig. 3.2 and the corresponding PLDOS analysis which will be shown in Fig. 3.5, the background shading is located at the same position in all graphs. Fig. 3.4 depicts a higher intensity of the superatomic S state compared to the case of the center doped species. The state is moreover is further away from the states that show high ligand interaction. This result is in good agreement with the observations made for the S state for the differently doped cluster cores. In the case of the surface doped $\mathrm{Au}_{12} \mathrm{Pd}^{2-}$ species the central atom $(\mathrm{Au})$ exhibits higher valency leading to less ligand involvement in creating the S state. 
On the other hand the P state located in the red shaded zone, shifts closer towards the gray shaded zone displaying higher ligand involvement due to fewer electrons that can be supplied from the surface atoms of the cluster core. The tunability of the role of the ligands in supporting the superatomic states is significant since a involvement of the ligands in forming the superatomic states and extending the orbitals over the ligands could have a positive effect on the ability of the clusters to interact electronically in assemblies.

Figure 3.5 depicts an overview of the atomic orbital contributions of the cluster core atoms of the differently doped cluster discussed in Fig. 3.2 to the bands. The PLDOS

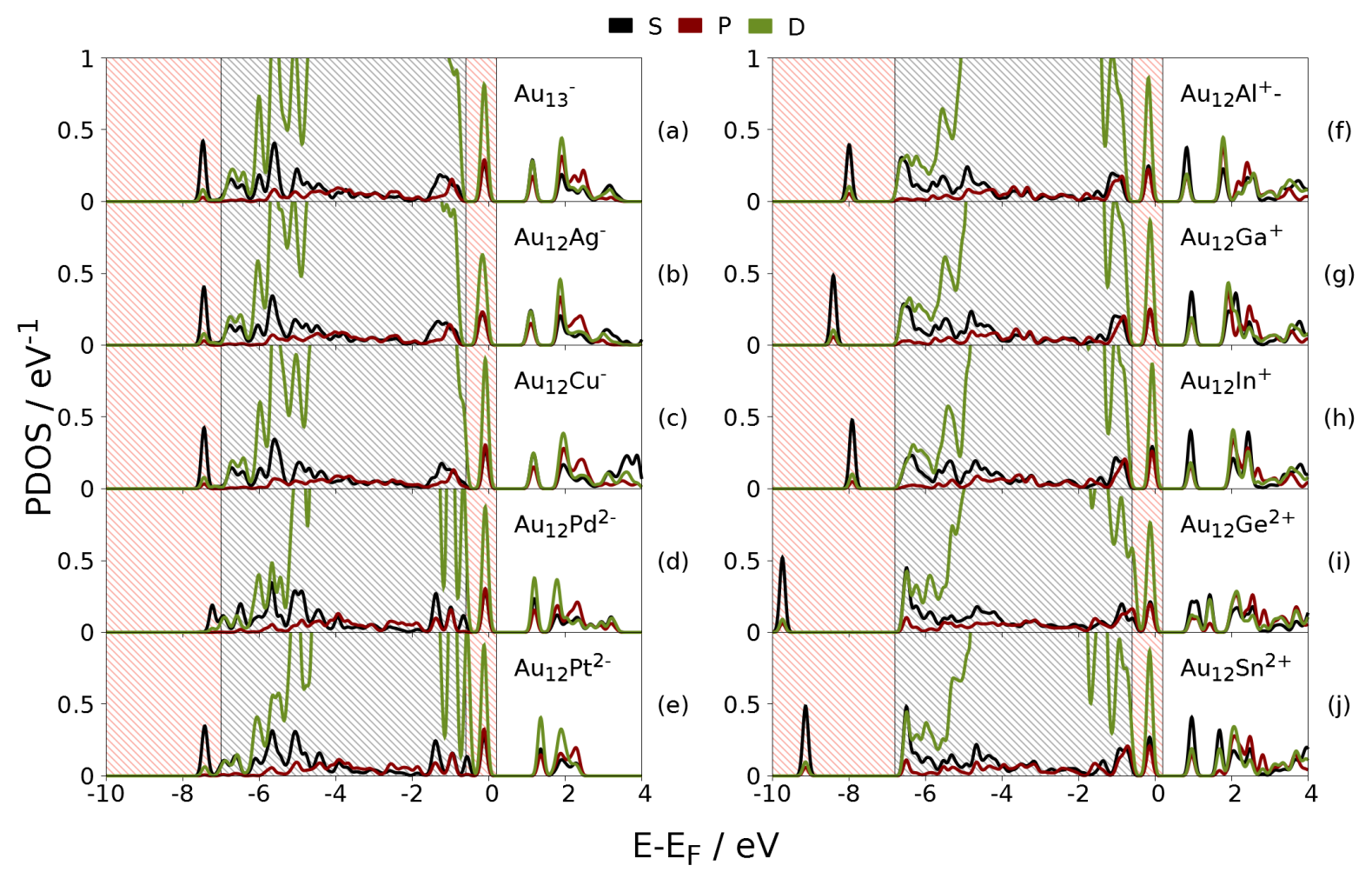

Figure 3.5: Visualization of the atomic orbital contributions to the bands of the differently doped $\mathrm{Au}_{13}\left[\mathrm{RS}(\mathrm{AuSR})_{2}\right]_{6}$ cluster cores exhibiting eight superatomic electrons, namely (a) $\mathrm{Au}_{13}^{-}$, (b) $\mathrm{Au}_{12} \mathrm{Ag}^{-}$, (c) $\mathrm{Au}_{12} \mathrm{Cu}^{-}$, (d) (b) $\mathrm{Au}_{12} \mathrm{Pd}^{2-}$, (e) $\mathrm{Au}_{12} \mathrm{Pt}^{2-}$, (f) $\mathrm{Au}_{12} \mathrm{Al}^{+}$, (g) $\mathrm{Au}_{12} \mathrm{Ga}^{+}$, (h) $\mathrm{Au}_{12} \mathrm{In}^{+}$, (i) $\mathrm{Au}_{12} \mathrm{Ge}^{2+}$, (j) $\mathrm{Au}_{12} \mathrm{Sn}^{2+}$.

analysis shows that the states highlighted with a gray colored background are to a large extent caused by the $5 d$ electrons of the cluster atoms and are therefore contributed 
to by ligand-cluster core interactions. Moreover, all S states are mostly contributed to by $s$ electrons. In contrast to the assumption that only the valence $s$ and $p$ electrons contribute to the formation of the superatomic states, a dominant $d$ contribution to the HOMO state can be found in all cases. This furthermore demonstrates the importance of the ligands in creating the superatomic electronic structure. The same can be found regarding the LUMO for the $\mathrm{Pd}$ and $\mathrm{Pt}$ doped species. In the case of the coinage metal clusters the s contribution to that state is slightly dominating, whereas it is dominating in all cases that consider the $p$-block doped cluster cores.

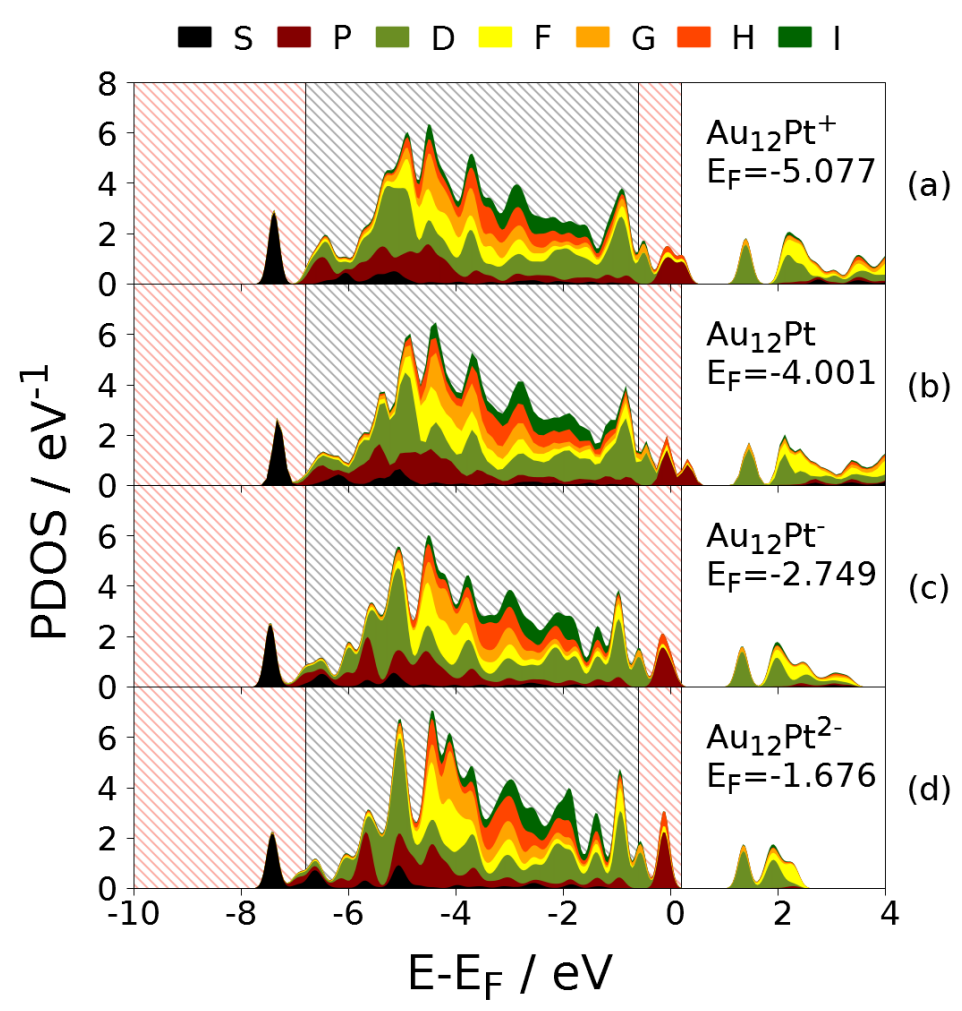

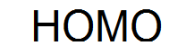
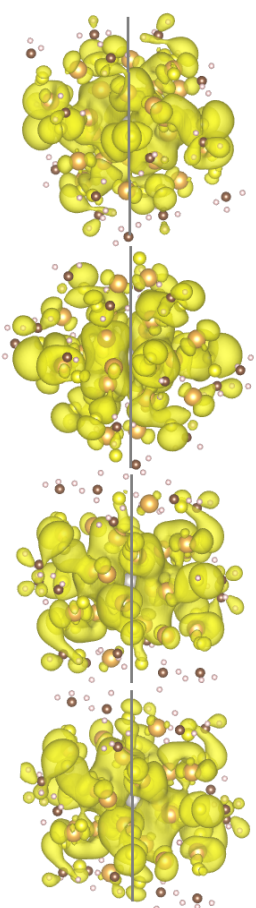

LUMO

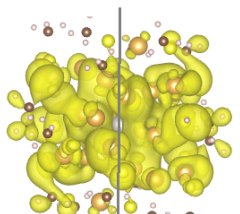

o 00
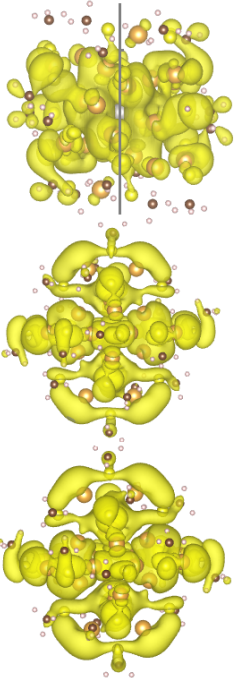

Figure 3.6: Visualization of the angular momentum projected density of states of the Pt doped cluster core with different charges: (a) 1+, (b) neutral (c) 1- and (d) 2- and the corresponding frontier orbitals with an isosurface cutoff of $0.0001 \mathrm{eV}$.

In order to depict the change of the superatomic electronic structure with cluster charge and to learn about the applicability of the superatom model to those clusters, the Pt doped cluster was chosen to visualize the overall trend, which was found to be similar 
in the case of all doped clusters. Fig 3.6 illustrates the PDOS and the corresponding frontier orbitals of the differently charged clusters with Pt doped cluster cores. According to the superatom model the $\mathrm{Au}_{12} \mathrm{Pt}^{+}$system should correspond to an electron count of five superatomic electrons, whereas the neutral cluster should in theory exhibit six superatomic delocalized electrons. $\mathrm{Au}_{12} \mathrm{Pt}^{-}$and $\mathrm{Au}_{12} \mathrm{Pt}^{2-}$ should be seven and eight superatomic electron systems, respectively. This presumption is indeed captured in Figure 3.6. The PDOS plot $(\mathrm{d})$ depicts an eight electron $\mathrm{P}$ shell closing with the $\mathrm{P}$ state as LUMO and the D state as HOMO. The corresponding $\mathrm{P}$ like LUMO orbital and D like HOMO are visualized on the right side of Fig. 5.6. In the case of the seven electron system in (c) the HOMO is shifted closer towards the Fermi energy resulting in a smaller HOMO-LUMO gap. The six superatomic electron exhibiting cluster (b) shows a splitting of the $\mathrm{P}$ state and therefore a small gap and a $\mathrm{P}$ like HOMO and LUMO orbital as visualized on the right hand side. Both $\mathrm{P}$ states are shifted closer towards one another in the case of (a) resulting in an even smaller HOMO-LUMO gap. This analysis shows that the superatom model is applicable to doped clusters with different charges and again reemphasizes the strong coupling between the cluster core and the ligands through the frontier orbital images. On the other hand it has been shown before that doping has an effect on the overall structure of the PDOS.

In order to further analyze the frontier orbitals of the doped clusters, the charge density of the HOMO orbital has been plotted as a function of distance from the origin of the clusters, with the central atom of the cluster chosen to be the origin. Figure 3.7.a displays the charge distribution of the HOMO orbitals of the clusters that should in theory exhibit eight delocalized superatomic electrons. On the other hand, Figure 3.7.b visualizes the charge distribution for the HOMO orbitals of the electron acceptor clusters (undoped, $\mathrm{Au}, \mathrm{Cu}, \mathrm{Pt}$ and Pd doped cluster cores) grouped for the six and five superatomic electron exhibiting clusters visualized in green and the seven and eight electron exhibiting clusters displayed in black. The same has been done for the clusters doped with $p$-block elements. Clusters consisting of six and five superatomic electrons are marked in red and the other group is marked in blue.

On closer inspection of graphic 3.7. a it can be seen that the charge density depicts three maxima indicated with dashed lines. The first maximum (labeled with "1") corresponds 
to the charge density around the central atom, the second indicates the charge density around the cluster core, whereas the third maximum corresponds to the charge density located around the gold atoms of the ligands. This again emphasizes the extension of the superatomic orbitals over the ligands and furthermore is in good agreement with the concept of the electrons being mainly delocalized around the cluster core. Moreover, it can be seen that the undoped cluster and the Pd doped species reveal a similar distribution. Most of the other doped clusters can be grouped together revealing lower maxima around the cluster core and the gold atoms of the ligands but more charge around the central atom and the outer part of the ligands. The Pt and Ge doped cluster cores seem to be outliers. To explain those findings it is necessary to consider the structural distortions of the clusters. Table 3 summarizes the distortion of the cluster cores away from a perfect icosahedron with different dopants and different charges. The degree of the distortion $c_{\text {dist }}$ is calculated as follows

$$
c_{\mathrm{dist}}=\frac{\sum_{\alpha}\left|1-\frac{\alpha}{\alpha_{\mathrm{set}}}\right|+\sum_{d}\left|1-\frac{d}{d_{\mathrm{av}}}\right|}{n_{\alpha}+n_{\mathrm{d}}},
$$

where $\alpha_{\text {set }}$ is the perfect icosahedron angle $\left(60^{\circ}\right), \alpha$ all measured angles, $d_{\mathrm{av}}$ the average distance of two neighboring atoms on the cluster core surface and $d$ all possible edge bond lengths. $n_{\alpha}$ and $n_{\mathrm{d}}$ represent the amount of measured angles and distances respectively. Table 3 captures all arising distortions due to charge, size of the dopant, Jahn-Teller

Table 3.1: Distortion of the cluster core structure away from a perfect icosahedron $c_{\text {dist }}$ listed for the differently doped clusters which exhibit five, six, seven or eight superatomic electrons.

\begin{tabular}{cccccc}
\hline \hline No. of superatomic $\mathrm{e}^{-}$ & $\mathrm{Au}_{13}$ & $\mathrm{Au}_{12} \mathrm{Ag}$ & $\mathrm{Au}_{12} \mathrm{Cu}$ & $\mathrm{Au}_{12} \mathrm{Pd}$ & $\mathrm{Au}_{12} \mathrm{Pt}$ \\
\hline \hline 8 & 0.025 & 0.030 & 0.034 & 0.018 & 0.012 \\
7 & 0.044 & 0.045 & 0.045 & 0.033 & 0.025 \\
6 & 0.096 & 0.089 & 0.081 & 0.065 & 0.051 \\
5 & 0.102 & 0.091 & 0.086 & 0.065 & 0.053 \\
\hline \hline${\text { No. of superatomic } \mathrm{e}^{-}}^{\mathrm{Au}} \mathrm{Au}_{12} \mathrm{Al}$ & $\mathrm{Au}_{12} \mathrm{Ga}$ & $\mathrm{Au}_{12} \mathrm{In}$ & $\mathrm{Au}_{12} \mathrm{Ge}$ & $\mathrm{Au}_{12} \mathrm{Sn}$ \\
\hline 8 & 0.036 & 0.035 & 0.052 & 0.074 & 0.043 \\
7 & 0.043 & 0.042 & 0.064 & 0.097 & 0.082 \\
6 & 0.056 & 0.057 & 0.086 & 0.128 & 0.120 \\
5 & 0.071 & 0.074 & 0.147 & 0.136 & 0.150 \\
\hline \hline
\end{tabular}




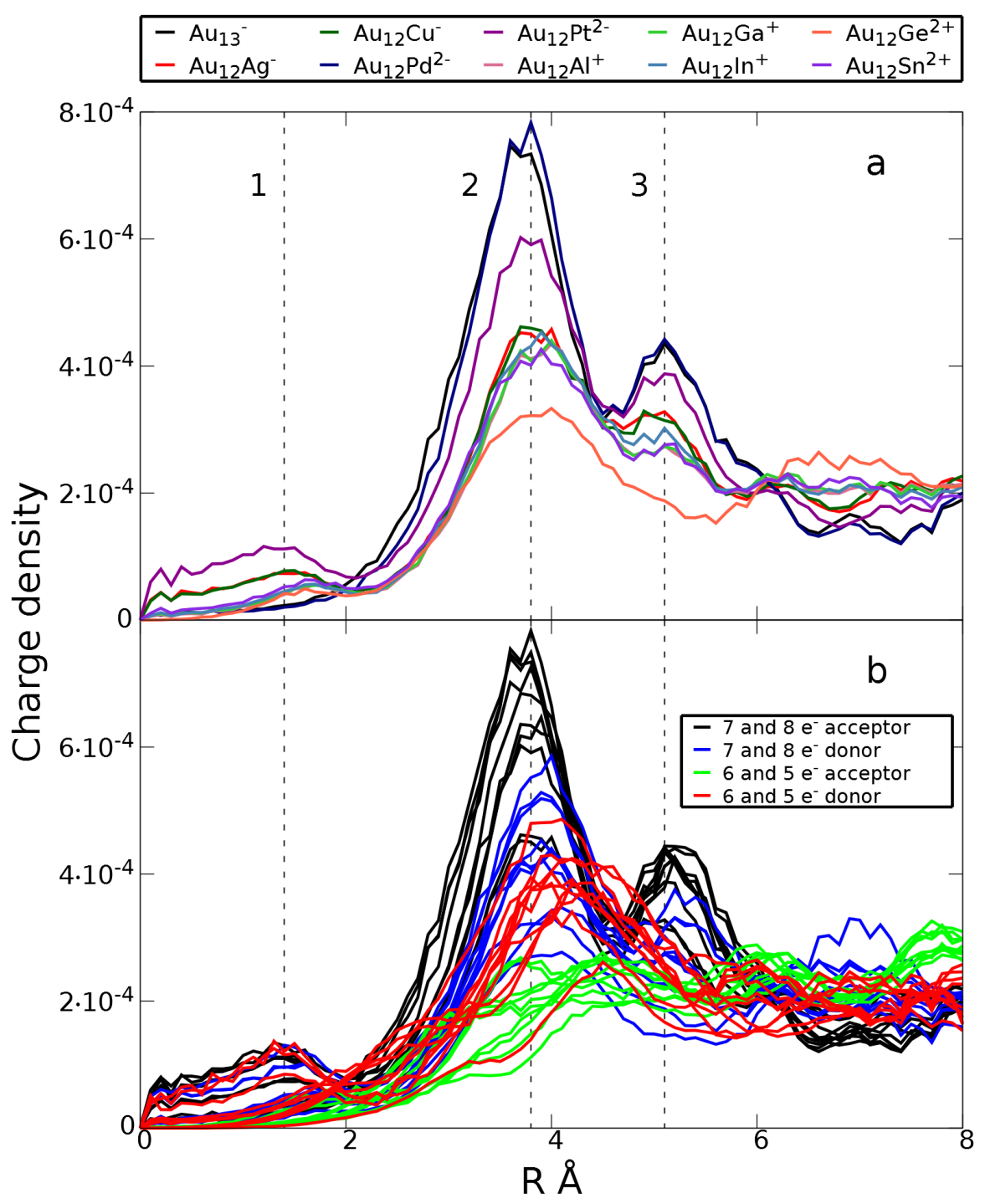

Figure 3.7: Charge density of the HOMO orbitals of the differently doped clusters plotted as a function of the distance from the central cluster core atom. The image a shows the charge density of all doped clusters that should in theory exhibit eight superatomic electrons. Whereas picture $\mathbf{b}$ shows the HOMO charge distribution for the five and six and the seven and eight electron systems grouped for the clusters that should in theory be electron donors or electron acceptors. 
distortion and the actual breaking of the icosahedral structure. Comparing the $c_{\text {dist }}$ values for the cluster cores exhibiting eight superatomic electrons to Figure 3.7.a shows that the charge density distribution of the HOMO orbitals visualized can be simply explained by structural distortion. The Pd doped cluster reveals a very similar distortion as the undoped cluster which in turn results in a similar charge density distribution. The cluster exhibiting the second closest distortion to the undoped species is the $\mathrm{Pt}$ doped cluster core resulting in a slightly shifted curve. The Ge doped cluster shows the largest distortion due to the small size of the dopant, resulting in a quite different trend of the plot. Thus, this analysis again shows that a single atom dopant has an influence on the superatomic structure caused by structural changes due to doping, and, that it is possible to vary the shape and extent of the superatomic orbitals as a consequence. Beyond the graphs visualized in Figure 3.7.a, Table 3.] helps to explain the findings depicted in image 3.7.b. Previous work has shown that the undoped cluster core, with either six, seven and eight superatomic electrons undergoes a Jahn-Teller distortion which is the strongest in the case of the six electron species ${ }^{[160]}$. This is in good agreement with the $c_{\text {dist }}$ calculated in this work. The cluster core distortion increases in all cases with fewer superatomic electrons. In the case of the electron acceptor clusters the distortion changes the most between the six and seven electron clusters. This can be related to a strong Jahn-Teller distortion, explaining the difference of the charge density distribution of the black and the green plots in Figure 3.7.b. Due to the large Jahn-Teller distortion occurring in the case of the five and six superatomic electron exhibiting clusters, the maxima discussed in the analysis of picture [3.7. a become less pronounced, and the spherical delocalization of the electrons around the cluster core becomes perturbed due to the shift away from a perfect icosahedron. This observation differs considering the electron donor clusters. In the case of the $p$-block metal doped cluster cores a steadily increasing distortion of the cluster core structure can be observed which corresponds to a overall small difference between the blue and red plots of the charge density distribution. Very large $c_{\text {dist }}$ are reached for a small amount of superatomic electrons in the case of the In, Ge and Sn doped cluster indicating that the icosahedral cage is falling apart. Another analysis of the electronic structure that has been performed, considers the charge of all these clusters. In Table 3.2 are listed the Bader charges for the cluster 
cores and the HOMO-LUMO gaps of the differently doped and charged clusters. Since these calculations have been performed spin unpolarized, the HOMO orbital is defined as a singly occupied orbital in the case of the open shell systems. It has been previously noted that the Bader charge results suggest that all additional charges are mainly located at the ligands since the charge of the cluster cores only changes in a small range with different overall cluster charges. This is in good agreement with previous

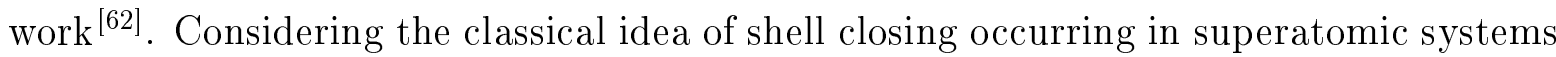
and therefore an integer increase or decrease of the delocalized electrons in the cluster core, a deviation between this common assumption and the actual observations arises. This lead us to analyze this change in detail. The metal core and the ligands are both contributing to the superatomic structure and behavior and are therefore strongly coupled. A clear separation of both fragments is not possible. The HOMO-LUMO gap values show as expected, that in all cases the eight superatomic electron exhibiting clusters reveal the largest gap and therefore the highest stability, due to the closed $\mathrm{P}$ shell. Moreover, it can be seen that doping leads to a smaller HOMO-LUMO gap, considering clusters with equal amount of spherically delocalized electrons, in most cases. However, doping with Pt and Pd leads to a larger gap and higher stability. A possible explanation for the increased stability due to doping could be given by the increased interaction energy between cluster core surface and central atom when doping with $\mathrm{Pt}$ or $\mathrm{Pd}[\mathbf{\square \times}]$. Furthermore the smallest icosahedral distortion is occurring considering those species, leading to the highest symmetry.

Figure 3.8 visualizes the correlation between the Bader charges, the expected superatomic valency and the HOMO-LUMO gap values given in Table B.2. The overall progression of the plots is consistent with the filling of superatomic orbitals. Going from a five superatomic electron species to six electrons, or from seven to a superatomic valency of eight, results in a small or negligible change of the HOMO-LUMO gap for all doped clusters. The rather small change is due to the fact that in both cases the same singly occupied P orbital is filled, and thus the characters of the HOMO and LUMO states do not change. The step from six to seven electrons however, leads to a significant increase of the HOMO-LUMO gap, as previously unoccupied P orbitals become singly occupied and the superatomic character of the LUMO changes from P to D. 
Table 3.2: Bader charges of the cluster cores and HOMO-LUMO gap of the differently charged and doped clusters all in theory exhibiting five, six, seven or eight superatomic electrons.

\begin{tabular}{|c|c|c|c|}
\hline cluster core & cluster charge & Bader charge & 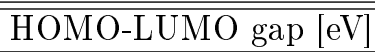 \\
\hline \multirow[t]{4}{*}{$\mathrm{Au}_{13}$} & $2+2+$ & 0.75 & ( 0.68 \\
\hline & $1+$ & 0.58 & 0.74 \\
\hline & 0 & 0.41 & 0.97 \\
\hline & $1-$ & 0.24 & 1.20 \\
\hline \multirow[t]{4}{*}{$\mathrm{Au}_{12} \mathrm{Ag}$} & $2+$ & 0.88 & 0.44 \\
\hline & $1+$ & 0.72 & 0.53 \\
\hline & 0 & 0.54 & 1.01 \\
\hline & $1-$ & 0.37 & 1.14 \\
\hline \multirow[t]{4}{*}{$\mathrm{Au}_{12} \mathrm{Cu}$} & $2+$ & 0.87 & 0.43 \\
\hline & $1+$ & 0.72 & 0.50 \\
\hline & 0 & 0.57 & 1.05 \\
\hline & 1- & 0.39 & 1.19 \\
\hline \multirow{4}{*}{$\mathrm{Au}_{12} \mathrm{Pd}$} & $1+$ & 0.57 & 0.52 \\
\hline & 0 & 0.41 & 0.63 \\
\hline & $1-$ & 0.23 & 1.02 \\
\hline & $2-$ & 0.06 & 1.23 \\
\hline \multirow[t]{4}{*}{$\mathrm{Au}_{12} \mathrm{Pt}$} & $1+$ & 0.58 & 0.23 \\
\hline & 0 & 0.43 & 0.38 \\
\hline & $1-$ & 0.24 & 1.30 \\
\hline & $2-$ & 0.05 & 1.47 \\
\hline \multirow[t]{4}{*}{$\mathrm{Au}_{12} \mathrm{Al}$} & $4+$ & 1.19 & 0.13 \\
\hline & $3+$ & 1.06 & 0.23 \\
\hline & $2+$ & 0.89 & 0.91 \\
\hline & $1+$ & 0.73 & 0.95 \\
\hline \multirow[t]{4}{*}{$\mathrm{Au}_{12} \mathrm{Ga}$} & $4+$ & 1.12 & 0.13 \\
\hline & $3+$ & 1.00 & 0.23 \\
\hline & $2+$ & 0.83 & 0.99 \\
\hline & $1+$ & 0.67 & 1.03 \\
\hline \multirow[t]{4}{*}{$\mathrm{Au}_{12} \mathrm{In}$} & $4+$ & 1.14 & 0.27 \\
\hline & $3+$ & 1.00 & 0.40 \\
\hline & $2+$ & 0.82 & 0.86 \\
\hline & $1+$ & 0.66 & 0.97 \\
\hline \multirow[t]{4}{*}{$\mathrm{Au}_{12} \mathrm{Ge}$} & $5+$ & 1.25 & 0.35 \\
\hline & $4+$ & 1.16 & 0.35 \\
\hline & $3+$ & 0.98 & 0.87 \\
\hline & $2+$ & 0.82 & 1.02 \\
\hline \multirow[t]{4}{*}{$\mathrm{Au}_{12} \mathrm{Sn}$} & $5+$ & 1.29 & 0.40 \\
\hline & $4+$ & 1.11 & 0.40 \\
\hline & $3+$ & 0.97 & 0.95 \\
\hline & $2+$ & 0.80 & 1.06 \\
\hline
\end{tabular}


In summary, all doped clusters seem to follow the expected trend in both pictures. The undoped gold cluster on the other hand, appear unique in its linear behavior. The HOMO-LUMO gap change between the six and seven and the seven and eight superatomic electron clusters is nearly equal, which might be explained by a large Jahn-Teller distortion stabilizing the six electron cluster. While the overall trend observed in this analysis is mostly consistent, a strong deviation form the behavior of the undoped cluster arises with doping and therefore chemical inhomogeneity. Thus, perturbations of the classical superatomic model arise through doping. In contrast to the perturbations of

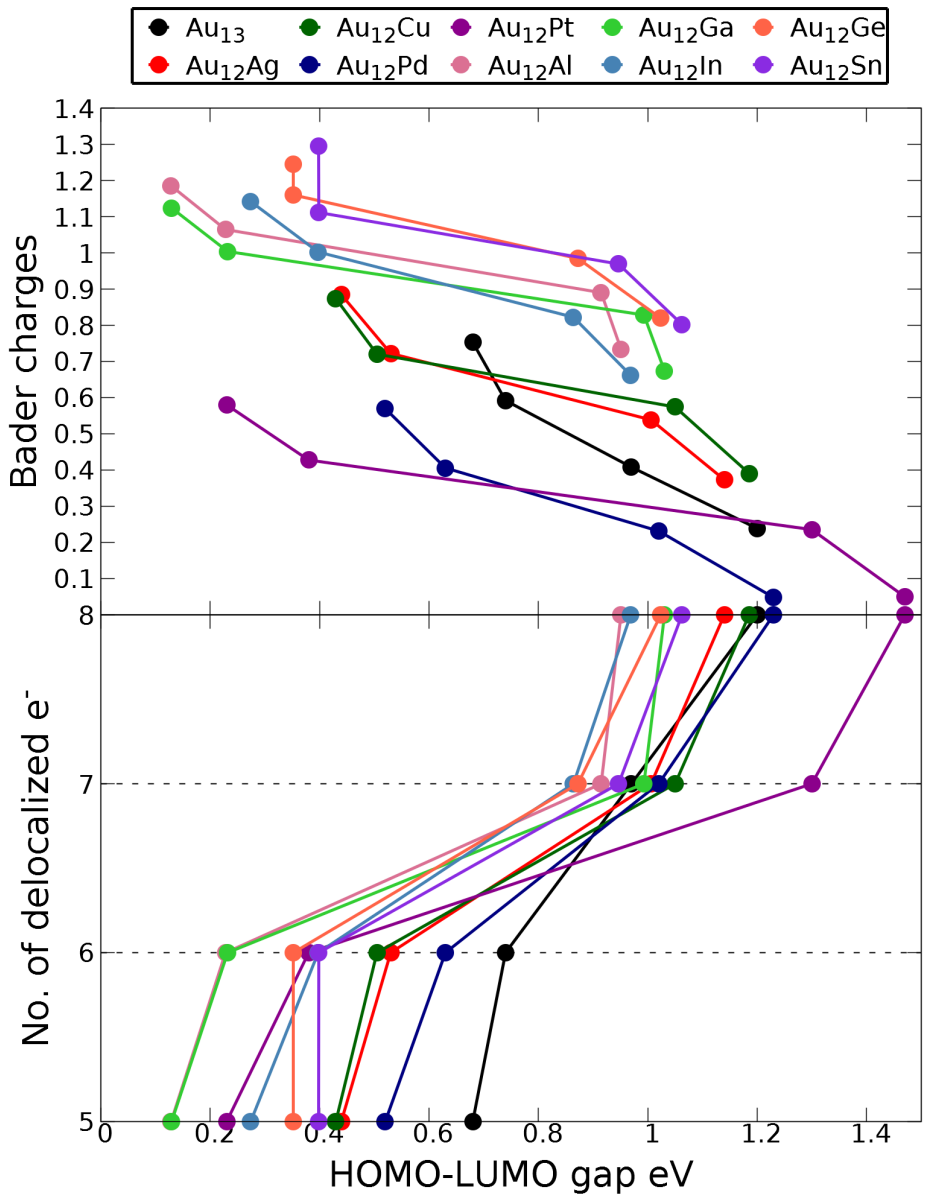

Figure 3.8: Bader charges of the cluster cores and the expected superatomic valency, plotted against the HOMO-LUMO gap for the differently doped and charged clusters.

the electronic structure arising from doping, Figure 3.9 and Table 3.3 show that simple 
counting of the expected superatomic valency helps to determine reasonable values for properties such as ionization potentials (IP) and electron affinities (EA) which on the other hand demonstrates the validity and robustness of the commonly used superatom model. The IP and EA values have all been calculated as the difference between total energies with the uncharged clusters as reference systems. The second, third etc. IPs or EAs concern a single electron, thus the first IP value has been subtracted from the difference of the total energy of the reference system and the total energy of the $2+$ charged species and so on. In Figure [3.9 are depicted the expected numbers of superatomic valency when removing or adding electrons against the absolute values of the corresponding IP/EA values. This visualization correlates very well with the superatomic model by

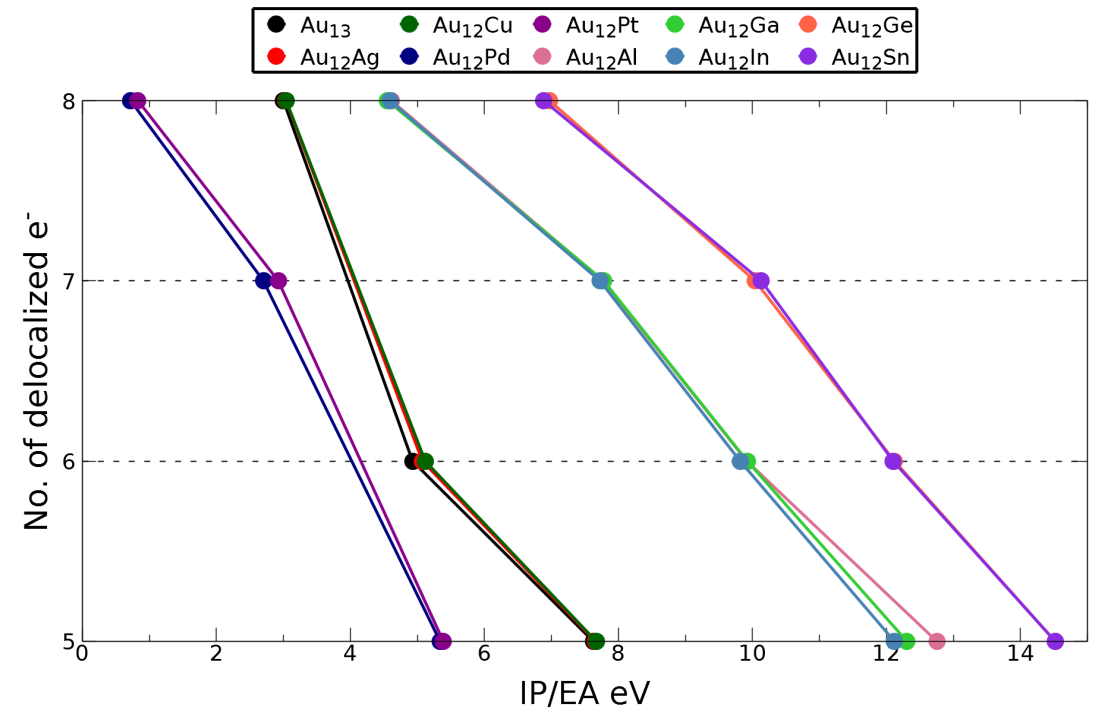

Figure 3.9: Number of delocalized superatomic electrons plotted against the absolute values of the ionisation potentials (IP) and electrons affinities (EA) corresponding to the number of electrons obtained. All values are calculated with the uncharged clusters as reference systems for all differently doped and charged clusters.

showing that the values for the ionisation potentials and electron affinities group by valencies of the cluster core, even though different degrees of structural distortions through size differences and positions of the dopants within the cluster core arise. The numbers in Figure 3.9 are summarized in Table 3.3 . The latter reveals, that the uncharged $\mathrm{Au}_{13}$, $\mathrm{Au}_{12} \mathrm{Ag}$ and $\mathrm{Au}_{12} \mathrm{Cu}$ clusters are seven electron systems and should therefore in their 
chemical behavior correspond to halogen atoms. In view of the electron affinities this is indeed the case since the first EA values calculated for those systems reflect the measured values of halogens $\left(-3.06--3.61 \mathrm{eV}^{[[70]}\right)$. The first EA values of the six electron $\mathrm{Au}_{12} \mathrm{Pd}$ and $\mathrm{Au}_{12} \mathrm{Pt}$ systems are smaller then those of the seven electron systems and therefore more consistent with the electron affinities of chalcogenes $(-2.08--1.46 \mathrm{eV}[\square \pi])$. Furthermore in this case the values for the second electron affinities are still negative, thus pointing out that a second charge is energetically favorable since it would lead to a $\mathrm{P}$ shell closing. On the other hand it is not possible to compare the IP values to

Table 3.3: Ionisation potentials and electron affinities for the differently doped clusters calculated with respect to the uncharged clusters.

\begin{tabular}{|c|c|c|c|c|c|}
\hline & $\mathrm{Au}_{13}$ & $\overline{\mathrm{Au}} \mathrm{u}_{12} \mathrm{Ag}$ & $\mathrm{Au}_{12} \mathrm{Cu}$ & $\mathrm{Au}_{12} \mathrm{Pd}$ & $\mathrm{Au}_{12} \mathrm{Pt}$ \\
\hline & \multicolumn{3}{|c|}{$S^{2} P^{5}$} & \multicolumn{2}{|c|}{$S^{2} P^{4}$} \\
\hline 1st IP & 4.93 & 5.08 & 5.12 & 5.34 & 5.39 \\
\hline 2nd IP & 7.63 & 7.66 & 7.67 & & \\
\hline 1st EA & -3.00 & -3.05 & -3.04 & -2.70 & -2.92 \\
\hline \multirow[t]{3}{*}{ 2nd EA } & & & & -0.72 & -0.83 \\
\hline & $\mathrm{Au}_{12} \mathrm{Al}$ & $\mathrm{Au}_{12} \mathrm{Ga}$ & $\mathrm{Au}_{12} \mathrm{In}$ & $\overline{\mathrm{Au}} \mathrm{u}_{12} \mathrm{Ge}$ & 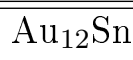 \\
\hline & \multicolumn{3}{|c|}{$S^{2} P^{6} D^{1}$} & \multicolumn{2}{|c|}{$S^{2} P^{6} D^{2}$} \\
\hline 1st IP & 4.61 & 4.55 & 4.59 & 4.69 & 4.69 \\
\hline 2nd IP & 7.77 & 7.78 & 7.72 & 6.97 & 6.89 \\
\hline 3rd IP & 9.92 & 9.92 & 9.82 & 10.04 & 10.13 \\
\hline 4th IP & 12.75 & 12.31 & 12.12 & 12.12 & 12.10 \\
\hline 5th IP & & & & 14.52 & 14.52 \\
\hline
\end{tabular}

those of individual atoms with similar valency. Nevertheless all ionization potentials are quite low in comparison to real atoms which can contribute in a positive way thinking of stable assemblies. A charge transfer from those clusters to another cluster seems to be easier accomplished when solely considering those values. Furthermore it is interesting that the first IP values all lie in the range of alkali metal and alkali earth metal atoms (e.g. $\left.\mathrm{Na}: 5.14 \mathrm{eV}, \mathrm{Ca}: 6.11 \mathrm{eV}, \mathrm{Li}: 5.39 \mathrm{eV}^{[\amalg \pi]}\right)$. Thus, combining one of the seven superatomic electron clusters with a $p$-block doped cluster could theoretically result in a $\mathrm{CsCl}$ or $\mathrm{NaCl}$ like behavior of the compound (see chapter $\mathbf{1}$ ).

Beyond that, there are some deviations of the IP values within a group of cluster cores of similar superatomic valency that can be explained by different polarizabilities of the 
differently doped clusters due to structural changes, since the IP and polarizability are inversely related ${ }^{[\amalg \pi,[\tau]}$. Moreover the high polarizability of the big cluster cores additionally explains that IP values are not comparable to individual atoms exhibiting similar valency. A good estimate of the polarizability increase and decrease for the acceptor clusters is given by a roughly calculated volume change of the cluster core which is calculated by averaging the distance of the core atom to the cluster core surface atoms and assuming a spherical shape, since it is the closest simple geometrical shape to an icosahedron. Figure 3.10 captures the change in volume graphically. Regarding the

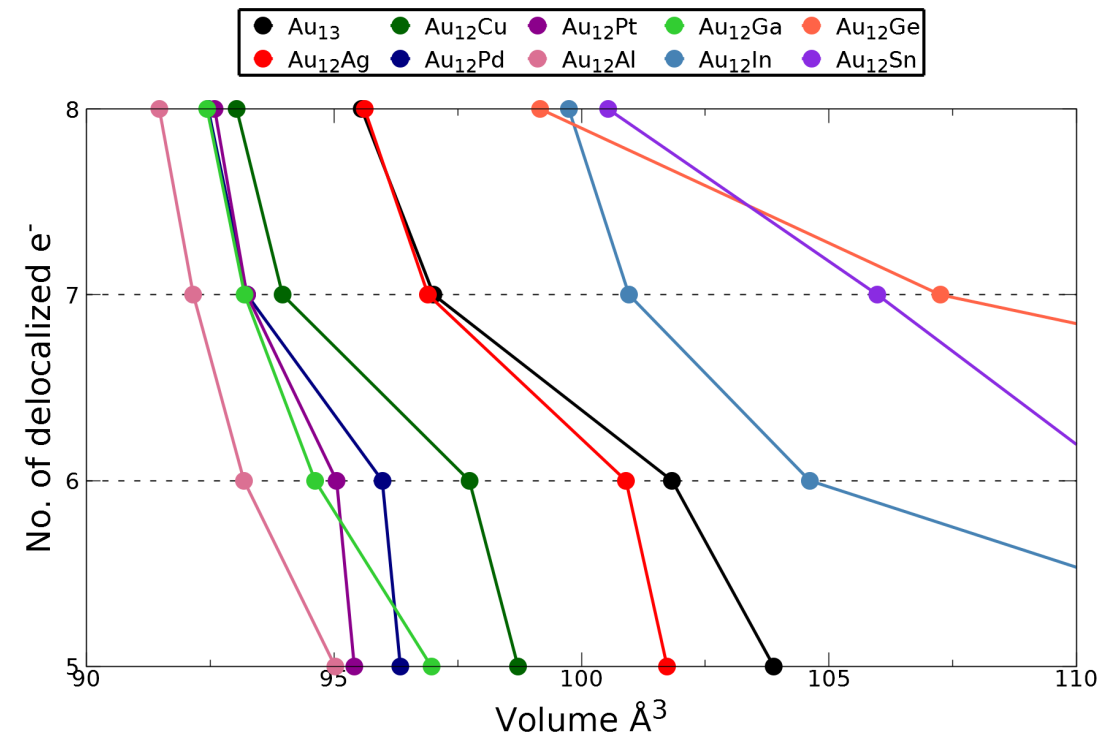

Figure 3.10: Theoretically expected number of superatomic deloclized electrons plotted against the volume of the cluster core, estimated by assuming a spherical shape.

acceptor clusters it can be seen that the relatively small change in volume caused by doping with Ag results in a smaller change in the IP value then it does when doping with $\mathrm{Cu}$. The $\mathrm{Cu}$ dopant causes a smaller cluster core volume and therefore a higher IP. The same can be seen when looking at Pd and Pt. Doping with Pt results in the smallest volume and therefore highest IP value. This proxy for the polarizability is not reliable in the case of the $p$-block metal doped clusters due to the larger distortions that lead further away from a perfect icosahedron, especially so in the case of Ge and Sn where the icosahedral structure is not maintained. 


\subsection{Conclusion}

The work presented in this chapter has demonstrated to what extent the superatomic model is applicable to single atom doped and differently charged clusters, and where perturbations or weaknesses of the model arise. Furthermore the knowledge gained about the electronic structure and the interplay between cluster core and ligands gives useful information, in particular, when thinking about the tunability of the superatomic electronic structure and in a next step about possible assemblies and therefore new functional materials.

The general angular momentum projected density of states analysis and the ionization potential and electron affinity discussion have shown that the commonly used superatom model is highly applicable to clusters revealing small chemical impurities and/or different charges. Changing the cluster charge while considering the valency of the dopant has resulted in the same amount of superatomic electrons, as predicted by the jellium model. The same simple electron count has led to the calculation of electron affinities for the differently doped clusters which resemble EA values of individual atoms with similar valency. The low ionization potential values in general and in particular of the $p$-block atom doped clusters immediately lead to the idea of using those clusters as building blocks. Especially since the coinage metal doped clusters reveal halogen like EAs and the $p$-block doped clusters alkali/alkali earth metal like IPs. That speaks in favor of the idea that binary assemblies of those clusters could theoretically result in $\mathrm{NaCl}$ and $\mathrm{CsCl}$ like structures with similar properties. Moreover this analysis reemphasizes the tunability of the electronic structure through small changes in the clusters composition and charge and furthermore gives some guidance to what is needed to be changed in order to obtain desired IP/EA values and a specific superatomic valency.

However, there are also significant perturbations of the superatomic model that arise. The individual atomic orbital contribution to the bands showed that there is some involvement in the $\mathrm{D}$ electrons in creating the superatomic states, also due to the fact that there is a large contribution of the ligands. Furthermore, doping of the cluster core causes large structural distortions e.g. Jahn-Teller distortions leading to different behavior of the cluster which can be seen in the Bader charge HOMO-LUMO plot and 
the discussion of the charge density distribution of the HOMO orbitals of the differently doped and charged clusters.

Another important finding is the strong coupling between the ligands and the clusters core which has been shown several times throughout this chapter. Moreover, it is remarkable how tunable the involvement of the ligands in creating those orbitals actually is. A large delocalized extent of the superatomic orbitals over the ligands can have a positive impact on the ability of the clusters to assemble in stoichiometric compounds. In this respect the influence of different ligands has to be further investigated. In summary the results obtained through this electronic structure analysis provide a good basis to think about potential cluster assemblies and how to design desired compounds. 


\section{Transition Metal Cluster Assemblies}

\subsection{Introduction}

Superatomic clusters have a wide range of potential applications. The creation of potential new functional materials based on superatom assemblies is one of the central ideas this research aims to address. A recent finding in this area was published in 2013 by Nuckolls et al. ${ }^{[96]}$. In cooperation with several other research groups, Nuckolls's group succeeded in synthesizing and characterizing compounds consisting of binary assemblies of phosphine protected transition metal-chalcogen clusters and fullerenes, namely $\left[\mathrm{Co}_{6} \mathrm{Se}_{8}\left(\mathrm{PEt}_{3}\right)_{6}\right]\left[\mathrm{C}_{60}\right]_{2},\left[\mathrm{Cr}_{6} \mathrm{Te}_{8}\left(\mathrm{PEt}_{3}\right)_{6}\right]\left[\mathrm{C}_{60}\right]_{2}$ and $\left[\mathrm{Ni}_{9} \mathrm{Te}_{6}\left(\mathrm{PEt}_{3}\right)_{8}\right]\left[\mathrm{C}_{60}\right]$. Those clusters assembled in structures that are known from individual atom based solids and furthermore show a charge transfer from the transition metal-chalcogen clusters to the fullerenes. $\left[\mathrm{Co}_{6} \mathrm{Se}_{8}\left(\mathrm{PEt}_{3}\right)_{6}\right]\left[\mathrm{C}_{60}\right]_{2}$ and $\left[\mathrm{Cr}_{6} \mathrm{Te}_{8}\left(\mathrm{PEt}_{3}\right)_{6}\right]\left[\mathrm{C}_{60}\right]_{2}$ were found to crystallize in a structure known from $\mathrm{CdI}_{2}$, whereas in the case of $\left[\mathrm{Ni}_{9} \mathrm{Te}_{6}\left(\mathrm{PEt}_{3}\right)_{8}\right]\left[\mathrm{C}_{60}\right]$ the nickel containing cluster is able to provide even more charge to the fullerene, resulting in a packing similar to rock-salt. Due to their atom based solid like characteristics these compounds were claimed to be "superatomic".

Since transition metals exhibit a very complicated electronic structure there is little knowledge about transition metal based clusters in the superatomic context. This chapter provides a detailed analysis of the mentioned individual transition metal-chalcogen clusters and furthermore tries to give some guidance towards whether or not the compounds produced by Nuckolls et al. resemble atom based solid state materials. The analysis is moreover contributing to the general understanding of those clusters in the 
superatomic framework and their potential application as building blocks.

The work presented in this chapter is based on Kohn-Sham density functional theory, while being aware of the limits arising from the methods used, in particular when it comes to transition metals and the prediction of spin states. Moreover, it has to be mentioned that the work presented in the following text passages has partially been carried out in cooperation with Lukas Hammerschmidt and has partially been published in Ref. [773]. The data presented in section 4.3.1] has solely been contributed to by the author of this thesis, while the remaining data was obtained in cooperation. Both are presented to enable discussion.

\subsection{Methods}

The calculations performed for this chapter have been conducted by using the program VASP. The input structures where obtained from ref. ${ }^{[96]}$.Considering the bulk calculations a full structure relaxation with respect to the symmetry has been performed in all cases. The Monkhorst-Pack k-grid was chosen to be a $4 \times 4 \times 4 \Gamma$-point centered grid whereas the plane wave energy cutoff was set to $520 \mathrm{eV}$. To ensure sufficient accuracy, the energy convergence criterion was set to $10^{-4} \mathrm{eV}$ and the ionic relaxation was stopped when all forces dropped below $0.01 \mathrm{eV} / \AA$. Moreover the Brillouin zone integration was done employing Gaussian smearing with a smearing factor of 0.05 since a smaller smearing factor has no influence on the minimum structure. For the analysis of the electronic properties (e.g. DOS, energy calculation and band structure) the k-grid was increased to $8 \times 8 \times 8$ and the smearing factor decreased to 0.01 .

For all three compounds different exchange correlation functionals have been tested namely, the local density approximation LDA ${ }^{[174]}$ and the generalized gradient approximations PBE ${ }^{[129,[30]}$ and PBEsol ${ }^{[175]}$. In order to include long range interactions regarding the PBE functional, Grimme's D3 dispersion correction [134] with Becke-Johnson damping ${ }^{\left[{ }^{[134]}\right.}$ (PBE-D3) as well as the Tkatchenko-Scheffler method (PBE-TS) ${ }^{[176]}$ have been included.

A similar procedure has been applied considering the calculation of the individual clusters, whereby the convergence criteria were set to $10^{-5} \mathrm{eV}$ regarding the total energy 
and to $0.05 \mathrm{eV} / \AA$ regarding the forces. The clusters where calculated in a cubic box of $35 \AA$ edge length, corresponding to a distance of at least $25 \AA$ between the particles. Furthermore, dipole and quadrupole corrections have been included.

For the detailed analysis of the individual clusters, the spin states of the clusters and the cluster fragments, as obtained from the VASP calculations, have been recalculated using the DFT code Amsterdam Density Functional (ADF) and the S12g functional by Marcel Swart ${ }^{[177]}$. For those calculations a small frozen core and a triple zeta basis set with 2 polarization functions ${ }^{[[78]}$ have been used. To account for relativistic effects the ZORA approximation [ [79-[81] was utilized. Subsequently, the obtained spin states were used to recalculate the clusters and cluster fragments with the program package VASP.

\subsection{Discussion}

The transition metal-chalcogen clusters produced and utilized by Nuckolls et al. to create the mentioned binary compounds, can be seen visualized in Fig. 4.7. For further

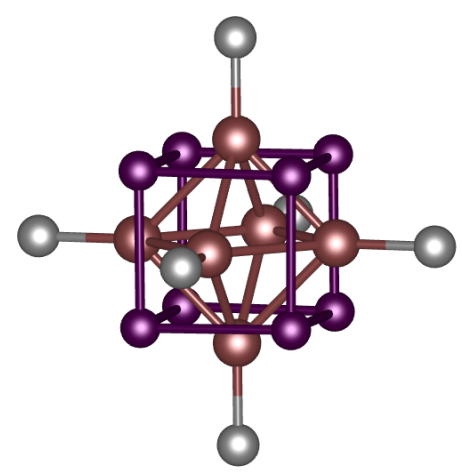

A

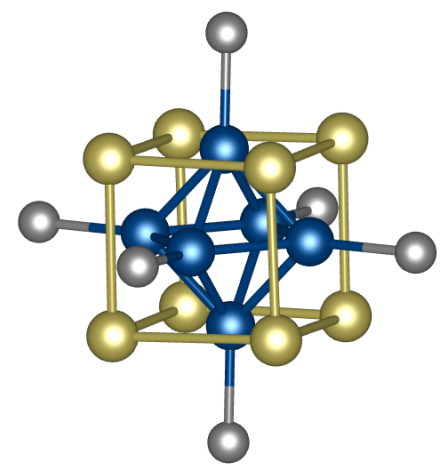

B

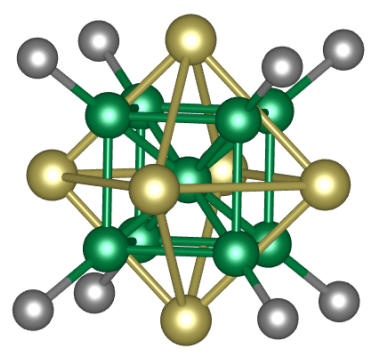

C

Figure 4.1: Schematic visualization of the different transition metal-chalcogen clusters utilized by Nuckolls et al. to create binary assemblies with $\mathrm{C}_{60}$. Color code: Co: light red, Se: violet, Cr: blue, Te: yellow, Ni: green and P: gray. For simplification the $\mathrm{C}$ and $\mathrm{H}$ atoms of the ligands have been neglected. A visualization of the structure of the cluster-fullerene assemblies can be found in Fig. ㄴ.7.

simplification compound $\left[\mathrm{Co}_{6} \mathrm{Se}_{8}\left(\mathrm{PEt}_{3}\right)_{6}\right]\left[\mathrm{C}_{60}\right]_{2}$ will in the following be referred to as compound $\mathrm{A},\left[\mathrm{Cr}_{6} \mathrm{Te}_{8}\left(\mathrm{PEt}_{3}\right)_{6}\right]\left[\mathrm{C}_{60}\right]_{2}$ as compound $\mathrm{B}$ and $\left[\mathrm{Ni}_{9} \mathrm{Te}_{6}\left(\mathrm{PEt}_{3}\right)_{8}\right]\left[\mathrm{C}_{60}\right]$ as com- 
pound C. Accordingly, cluster $\left[\mathrm{Co}_{6} \mathrm{Se}_{8}\left(\mathrm{PEt}_{3}\right)_{6}\right]$ will be labeled cluster A, $\left[\mathrm{Cr}_{6} \mathrm{Te}_{8}\left(\mathrm{PEt}_{3}\right)_{6}\right]$ cluster B etc.

In order to get a first impression about the agreement between experiment and theory, the calculated lattice parameters for all three compounds have been compared to experimental data. Table 4.0 lists the experimental lattice parameters taken from Ref. [06] the parameters estimated with different functionals and their deviation from the experiment in percent. Comparing the computed to the experimental values, depicts a very

Table 4.1: Comparison of the experimental and computational lattice parameters for the compounds: A: $\left[\mathrm{Co}_{6} \mathrm{Se}_{8}\left(\mathrm{PEt}_{3}\right)_{6}\right] \mathrm{B}:\left[\mathrm{C}_{60}\right]_{2}, \quad\left[\mathrm{Cr}_{6} \mathrm{Te}_{8}\left(\mathrm{PEt}_{3}\right)_{6}\right]\left[\mathrm{C}_{60}\right]_{2}$ and $\mathrm{C}$ : $\left[\mathrm{Ni}_{9} \mathrm{Te}_{6}\left(\mathrm{PEt}_{3}\right)_{8}\right]\left[\mathrm{C}_{60}\right] . \Delta \mathrm{a}, \Delta \mathrm{b}$ and $\Delta \mathrm{c}$ give the deviation of the theoretical lattice parameters from the experimentally obtained values in percentage.

\begin{tabular}{|c|c|c|c|c|c|}
\hline & $\mathrm{a}[\AA]$ & $\mathrm{c}[\AA]$ & $\Delta \mathrm{a}[\%]$ & $\Delta \mathrm{c}[\%]$ & $\Delta \mathrm{V}[\%]$ \\
\hline \multicolumn{6}{|c|}{ "Compound A } \\
\hline Experiment & 15.750 & 12.479 & & & \\
\hline LDA & 15.556 & 12.057 & -1.23 & -3.38 & -5.75 \\
\hline PBE & 16.613 & 13.634 & 5.48 & 5.48 & 21.55 \\
\hline PBE-D3 & 15.845 & 13.032 & 0.60 & 4.43 & 5.68 \\
\hline PBE-TS & 15.844 & 12.753 & 0.59 & 2.19 & 1.41 \\
\hline PBEsol & 16.058 & 13.006 & 1.69 & 4.22 & 8.34 \\
\hline \multicolumn{6}{|c|}{ Compound B } \\
\hline Experiment & 16.182 & 12.358 & & & \\
\hline LDA & 15.650 & 12.035 & -3.29 & -2.61 & -8.91 \\
\hline PBE & 16.872 & 13.017 & 4.27 & 5.33 & 14.51 \\
\hline PBE-D3 & 16.064 & 12.184 & -0.73 & -1.41 & -2.84 \\
\hline PBE-TS & 16.004 & 12.130 & -1.10 & -1.85 & -4.00 \\
\hline PBEsol & 16.254 & 12.446 & 0.45 & 0.71 & 1.61 \\
\hline \multicolumn{6}{|c|}{ Compound $\mathrm{C}$} \\
\hline Experiment & 21.662 & & & & \\
\hline LDA & 21.712 & & 0.23 & & 0.69 \\
\hline PBE & 22.458 & & 3.67 & & 11.43 \\
\hline PBE-D3 & 21.696 & & 0.16 & & 0.47 \\
\hline PBE-TS & 21.667 & & 0.02 & & 0.07 \\
\hline PBEsol & 21.774 & & 0.52 & & 1.55 \\
\hline
\end{tabular}

good agreement of the dispersion corrected PBE parameters with the experiment. This already indicates that long range interactions play an important role in all three systems. The best agreement between computational and experimental data can be found in the case of compound C. Even PBEsol is found to perform well with respect to the 
investigated parameters. Moreover, Table 4.7 reveals that even though LDA tends to overestimate the binding and therefore to underestimate the lattice parameters (which is at least the case for compound A and B), the overall performance of LDA in predicting the structural properties is better then in the case of PBE. The overestimation of the binding in the case of LDA compensates somewhat for the missing long range interactions leading to a better agreement with experiment. Thus, the LDA derived structures seem like a suitable choice for further investigation of the electronic properties.

Subsequently, the binding situation of the compounds has been investigated. In order to understand whether or not the interaction between the clusters and fullerenes can be compared to atom based bulk materials. Cohesive energies have been computed as the difference between the total energy of the compound and the total energies of the free building blocks $E_{\text {cohesive }}=E_{\text {compound }}-\left(E_{\text {cluster }}+n E_{\mathrm{C}_{60}}\right)$, with $n=2$ in the case of compound $\mathrm{A}$ and $\mathrm{B}$ and $n=1$ in the case of compound C. Furthermore, Bader charges have been calculated to capture a potential charge transfer between the transition metalchalogen clusters and the fullerenes. The cohesive energies $E_{\text {coh }}$ and Bader charge values q, obtained using different functionals, are summarized in Table 4.2. The Bader charges

Table 4.2: Cohesive energies $E_{\text {coh }}$ calculated as the difference of the total electronic energy of the whole assembly subtracted by the energies of the individual clusters of each unit cell (relaxed in vacuum) for the three different compounds A: $\left[\mathrm{Co}_{6} \mathrm{Se}_{8}\left(\mathrm{PEt}_{3}\right)_{6}\right]\left[\mathrm{C}_{60}\right]_{2}$, B: $\left[\mathrm{Cr}_{6} \mathrm{Te}_{8}\left(\mathrm{PEt}_{3}\right)_{6}\right]\left[\mathrm{C}_{60}\right]_{2}$ and $\mathrm{C}:\left[\mathrm{Ni}_{9} \mathrm{Te}_{6}\left(\mathrm{PEt}_{3}\right)_{8}\right]\left[\mathrm{C}_{60}\right]$. The table additionally includes the Bader charges of the metal-chalogen clusters $q$ in each compound.

\begin{tabular}{ccccccc}
\hline \hline & \multicolumn{2}{c}{ Compound A } & \multicolumn{2}{c}{ Compound B } & \multicolumn{2}{c}{ Compound C } \\
\hline & $E_{\text {coh }}[\mathrm{eV}]$ & $q[\mathrm{e}]$ & $E_{\text {coh }}[\mathrm{eV}]$ & $q[\mathrm{e}]$ & $E_{\text {coh }}[\mathrm{eV}]$ & $q[\mathrm{e}]$ \\
\hline LDA & -8.18 & 0.86 & -4.60 & 0.55 & -5.21 & 1.49 \\
PBE & -0.40 & 0.70 & -0.43 & 0.49 & -1.66 & 1.30 \\
PBE-D3 & -6.06 & 0.76 & -7.24 & 0.51 & -5.74 & 1.46 \\
PBE-TS & -8.88 & 0.80 & -10.26 & 0.50 & -7.44 & 1.42 \\
PBEsol & -0.45 & 0.72 & -0.52 & 0.51 & -2.14 & 1.45 \\
\hline \hline
\end{tabular}

depict a charge transfer from the transition metal-chalcogen clusters to the $\mathrm{C}_{60} \mathrm{~S}$ in all cases, thus reflect the experimental results. Nuckolls et al. observed, that cluster A and $\mathrm{B}$ are able to roughly transfer one electron to each $\mathrm{C}_{60}$, whereas cluster $\mathrm{C}$ is able to transfer even more charge. Moreover, it can be taken from Table 4.2, that the variation of the Bader charges is narrow considering all functionals. This strengthens the validity 
of the calculated charge trends.

In order to understand the role of the ligands in the occurring charge transfer, compound $\mathrm{A}$ has been recalculated utilizing the functional PBE and fully relaxed without the ligand shell. The Bader charge of the cluster dropped to 0.03, thus nearly no charge transfer to the $\mathrm{C}_{60}$ could been observed. This drastic change suggests that the ligands act as a dielectric medium, controlling the overall charge and charge distribution of the cluster. It furthermore reinforces the importance of the ligands when it comes to the electronic structure, as it has been shown in the case of the thiolate protected gold clusters in chapter 3 . Similar to those gold clusters, any charge added to or removed from the free transition metal-chalcogen cluster (A as well as B and C) is found to mainly be located at the ligands.

Regarding the cohesive energies listed in Table 4.2 no experimental data is available for comparison. Nevertheless, those purely theoretical values contain valuable information about the interactions present. Comparing the PBE calculated binding energies with the energies obtained including additional dispersion corrections, reveals that the main component contributing can be identified as van der Waals interactions. The pure PBE results tend to overestimate the lattice parameters and therefore to underestimate the binding energy, whereas taking long range interactions into account leads to a much larger binding energy. Comparing the dispersion interaction for all three compounds, shows that the dispersion contribution to $E_{\text {coh }}$ is larger in the case of compound $A$ and $\mathrm{B}$ than it is for compound $\mathrm{C}$, which is simply due to the fact that $\mathrm{A}$ and $\mathrm{B}$ crystallize in a $\mathrm{CdI}_{2}$ type structure. $\mathrm{CdI}_{2}$ has a layered structure where van der Waals forces between the anion layers ensure the stability of the crystal.

Beyond PBE, energies utilizing other functionals have been computed. PBEsol for example gives similar results to PBE since it is nothing but the PBE functional adjusted for the prediction of structural properties. LDA however overestimates the binding energy due to underestimating the lattice parameters and therefore provides results that are in good agreement with the dispersion corrected PBE results. Due to the fact that the electronic properties cannot be calculated considering the dispersion correction, LDA appears to be a suitable functional with which to derive the electronic properties of the bulk. 
So far the analysis has shown, that all compounds are dominated by long range interactions. Nevertheless there are binding contributions present that go beyond van der Waals interactions, e.g. a non zero value for $E_{\text {coh }}$ can be found regarding the PBE calculations and furthermore a charge transfer between the transition metal-chalcogen clusters and the fullerenes can be observed. Those additional contributions strengthen the idea of an atom based solid state material resembling behavior and therefore a superatomic structure of the compounds.

\subsubsection{Electronic Structure}

In the previous passages it has been mentioned that the LDA results are due to error cancellation in good agreement with the dispersion corrected PBE values. Thus, the LDA functional has been used to derive the electronic properties presented in the following section.

Fig. 42 shows the computed band structure and angular momentum projected density of states for all three compounds close to the Fermi level, plotted for alpha and beta spin separately. The previous analysis has shown that the interaction between the clusters goes beyond pure van der Waals forces, this assumption is reinforced by the band structure. In contrast to the flat bands expected in purely van der Waals contributed solids like molecular solids, weakly disperse bands can be found in all three cases revealing collective electronic behavior of the compound. In addition compound $\mathrm{A}$ and $\mathrm{B}$ are found to exhibit a band gap of 50-100 meV which is in good agreement with the experiments conducted by Nuckolls et al., showing that both materials are small gap semiconductors.

On the other hand however, in general all states show a very localized nature. Moreover in the case of compound A and B the highest occupied band is contributed by the $p$ and $d$ orbitals of the transition metal-chalcogen cluster and therefore not by the anion (here $\mathrm{C}_{60}$ ), as it would be expected by an ionic solid. This suggests a rather small ionic character of the compound.

In contrast to compound $\mathrm{A}$ and $\mathrm{B}$, the nickel containing compound $\mathrm{C}$ shows some indication of something like an actual covalent bond between cluster and fullerene. Table 


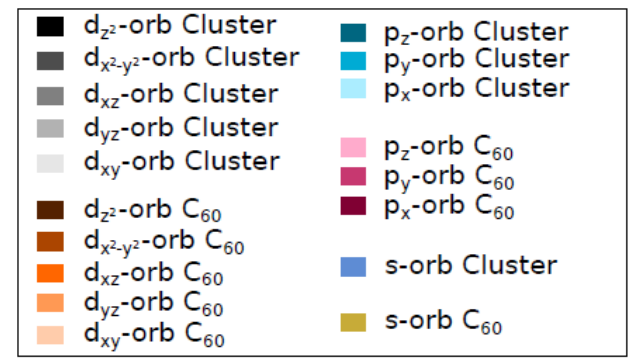
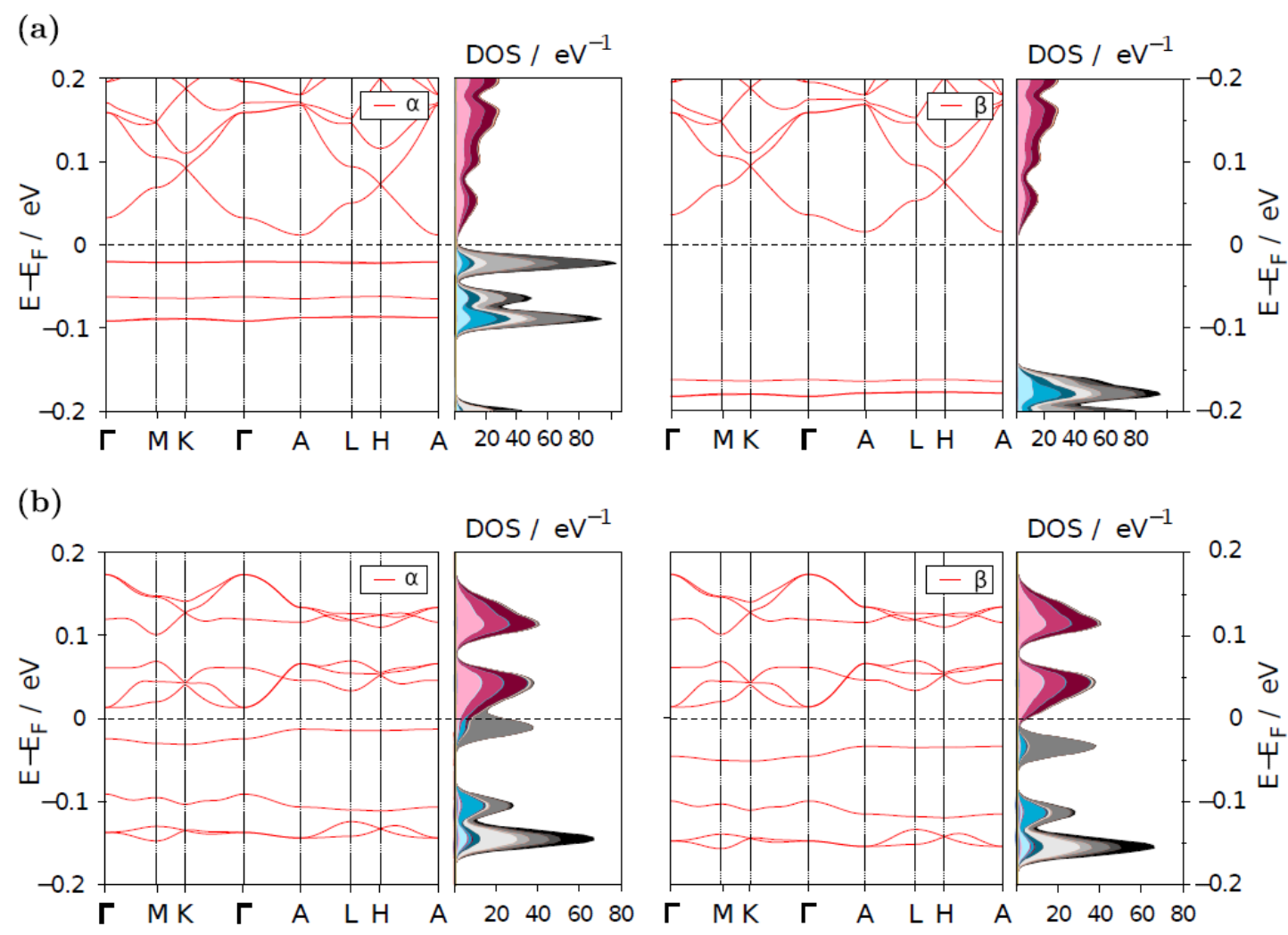

(c)
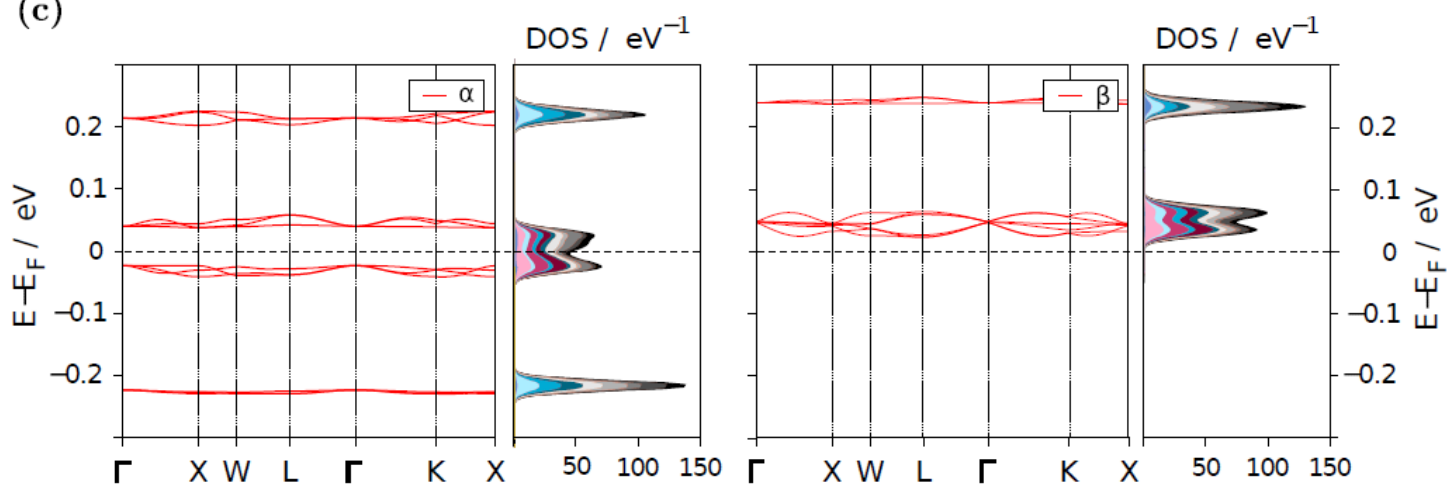

Figure 4.2: Calculated band structure and angular momentum projected density of states close to the Fermi level for the three different compounds: A: $\left[\mathrm{Co}_{6} \mathrm{Se}_{8}\left(\mathrm{PEt}_{3}\right)_{6}\right]\left[\mathrm{C}_{60}\right]_{2}$, B: $\left[\mathrm{Cr}_{6} \mathrm{Te}_{8}\left(\mathrm{PEt}_{3}\right)_{6}\right]\left[\mathrm{C}_{60}\right]_{2}$ and $\mathrm{C}:\left[\mathrm{Ni}_{9} \mathrm{Te}_{6}\left(\mathrm{PEt}_{3}\right)_{8}\right]\left[\mathrm{C}_{60}\right]$. The Figure is reproduce from Ref. ${ }^{[173]}$ with permission. 
42 already revealed a larger binding contribution beyond van der Waals interactions in the case of compound $\mathrm{C}$, than found for A and B. Taking a look at the band structure of compound $\mathrm{C}$ and the angular momentum projected density of states, even shows that the bands close to the Fermi level consist of a hybridization of cluster and fullerene $p$ and $d$ orbitals. Moreover, it can be seen that a Jahn-Teller distortion leads to a splitting of the states and therefore to three occupied and three unoccupied bands for the alpha spin. In the case of the beta spin the highest occupied bands can be found at much lower energies, solely contributed by transition metal-chalcogen cluster orbitals. This theoretical observation is in good agreement with the experiment, since compound $\mathrm{C}$ was experimentally found to reveal a magnetic ground state at low temperatures ${ }^{[96]}$. Another type of analysis that can be found in Ref. ${ }^{[173]}$ and allows direct comparison with experiment, is the estimation of the Pauli magnetic susceptibility of compound A and $\mathrm{B}$ derived from the spin unpolarized PBE band structure within the framework of Boltzmann theory using the Botztrap code ${ }^{[182]}$. Nuckolls et al. showed that compound $\mathrm{A}$ is paramagnetic and reveals a temperature independent magnetic moment, whereas compound B was found to depict a more complex magnetic structure.

(a)
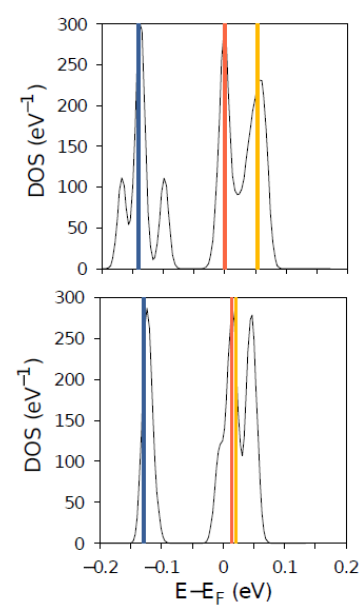

(b)
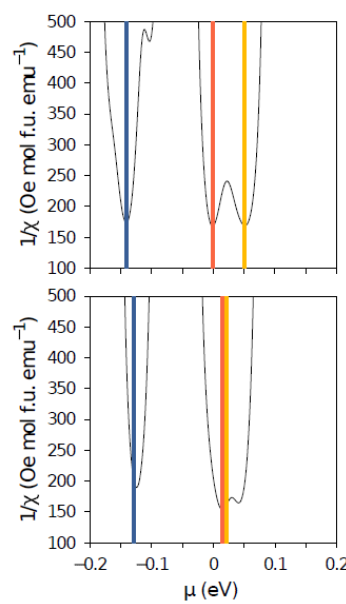

(c)
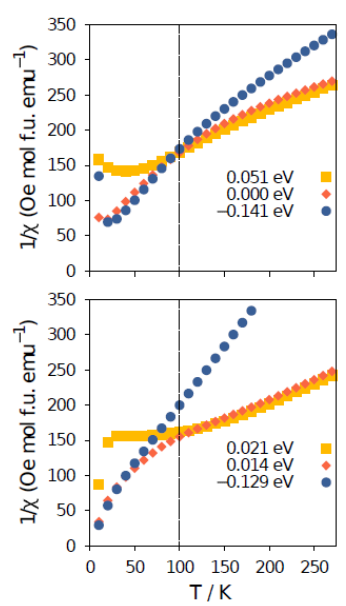

Figure 4.3: (a): Density of states, (b) magnetic susceptibility as function of the chemical potential and (c) the magnetic susceptibility as function of the the temperature for compounds $\left[\mathrm{Co}_{6} \mathrm{Se}_{8}\left(\mathrm{PEt}_{3}\right)_{6}\right]\left[\mathrm{C}_{60}\right]_{2}$ (top) and $\left[\mathrm{Cr}_{6} \mathrm{Te}_{8}\left(\mathrm{PEt}_{3}\right)_{6}\right]\left[\mathrm{C}_{60}\right]_{2}$ (bottom), derived within semi classical Botzmann transport theory. The Figure is reproduced from Ref. ${ }^{[173]}$ with permission. 
Figure 4.3. a shows the density of states, 4.3. $\mathrm{b}$ the chemical potential dependent inverse magnetic moment at $100 \mathrm{~K}$ and graphic 4.3. netic moment at a given chemical potential $\mu$ reflecting different $n-$ and $p$ - dopings. The figures at the top correspond to compound A, while the bottom figures reflect the trends found for compound B. Even though the calculations are done within rough approximations a good agreement with the experimental observations is realized. Comparing the results visualized in Fig. 4.3.c to experimental data reveals that the temperature dependence of the magnetic moment of compound $\mathrm{A}$ is well captured for small or no p-doping in the high temperature regime. In the case of compound B the curve obtained for small p-doping in the low temperature regime reflects the progression of the experimentally obtained curve but still remains temperature dependent for higher temperatures. The latter is in contrast to experiment a plateau is reached after a certain temperature. Recapitulating the investigation of the magnetic properties depicts the possibility to derive collective properties for the compounds based on calculated band structures ${ }^{[173]}$, again supporting the idea of an underlying superatomic structure.

In summary this section has again shown, that all compounds are dominated by weak long range interactions and exhibit a weak ionic character. However the idea of an superatomic or atom like compound has been reinforced by showing that the interactions between the compound building blocks go beyond a pure van der Waals contribution. Weakly disperse bands can be found in all cases. Regarding compound C a hybridization of $p$ and $d$-orbitals of the clusters and the fullerenes even indicates the formation of a covalent bond. Furthermore collective magnetic properties have been found to emerge from the compounds.

\subsubsection{Superatomic Structure}

In order to estimate whether or not the three discussed compounds can been seen as atom like solid-state materials it is necessary to investigate the individual building blocks regarding their superatomic character. To do so the free clusters have been relaxed in vacuum as explained in section 6.2. Since the spin state of a cluster can have an impact on its superatomic nature, the PBE obtained structures/substructures have in a next 
step been used to obtain the spin state utilizing the program package ADF.

Fig. 4.4.d shows a schematic visualization of the cluster $\mathrm{Ni}_{9} \mathrm{Te}_{6}\left(\mathrm{PEt}_{3}\right)_{8}$ and its investigated fragments. Image 4.4. a depicts the chalcogen substructure, 4.4.b the transition metal cluster, 4.4.c both fragments combined and 4.4.d the whole cluster including ligands. Each fragment has been analyzed by itself in the structure obtained from the whole cluster relaxation. The whole procedure has been repeated for the clusters $\mathrm{Co}_{6} \mathrm{Se}_{8}\left(\mathrm{PEt}_{3}\right)_{6}$ and $\mathrm{Cr}_{6} \mathrm{Te}_{8}\left(\mathrm{PEt}_{3}\right)_{6}$. Substructure $\mathrm{A}$ consists in all three investigated

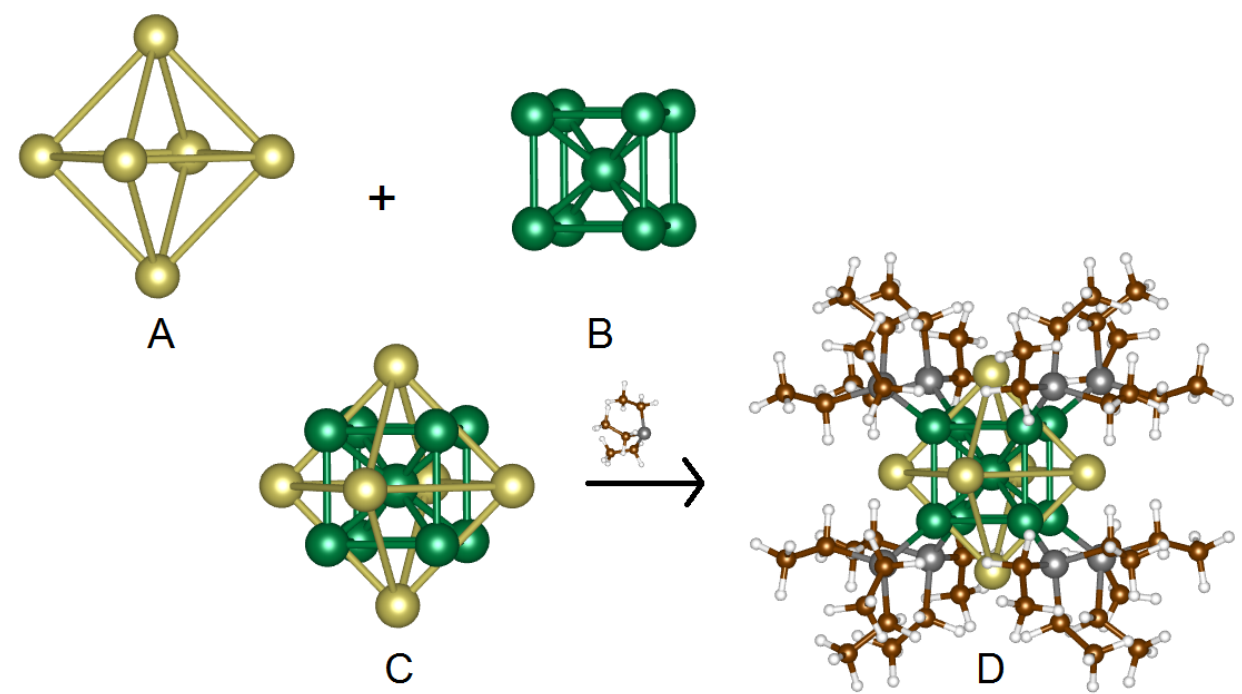

Figure 4.4: Schematic visualization of the $\mathrm{Ni}_{9} \mathrm{Te}_{6}\left(\mathrm{PEt}_{3}\right)_{8}$ cluster and the separately investigated substructures (A: chalcogen cluster, B: transition-metal cluster, C: mixed cluster, D: whole cluster including ligands ). The substructure investigation has analogously been repeated for cluster $\mathrm{Co}_{6} \mathrm{Se}_{8}\left(\mathrm{PEt}_{3}\right)_{6}$ and $\mathrm{Cr}_{6} \mathrm{Te}_{8}\left(\mathrm{PEt}_{3}\right)_{6}$.

clusters of a chalcogen cluster exhibiting six to eight atoms, namely a $\mathrm{Te}_{6}, \mathrm{Te}_{8}$ and $\mathrm{Se}_{8}$ cluster. The eight atom containing clusters have a cubic shape (see fig. 4. d), whereas the $\mathrm{Te}_{6}$ can be seen visualized in Figure 4.4. The superatomic character of these clusters has been investigated using several tools such as the PDOS, the PLDOS, the visualization of the orbitals and the electron localization function (ELF) ${ }^{[[83]}$. Fig. 4.5 shows the PDOS and PLDOS including selected orbital visualization for the $\mathrm{Te}_{8}$ cluster which is a fragment of $\mathrm{Cr}_{6} \mathrm{Te}_{8}\left(\mathrm{PEt}_{3}\right)_{6}$. Taking a look at the PDOS depicts states with a clear angular momentum character that are usually used to show superatomic states. In this case however, the large splitting of the states that are caused by $s$ electrons and the 
ones contributed to by $p$ electrons, as visualized in the PLDOS, indicates that all states are individual atomic states rather then superatomic states. This is reinforced by the visualization of selected states revealing that the low energy states are made up by atomic $s$ orbitals and the states found at higher energy by $p$ orbitals. In addition the non-superatomic character of this cluster fragment is again reemphasized by Figure 4.6 depicting a visualization of the ELF with a value of 0.5 to 0.7 ( 1 is considered to reflect perfect localization). Fig. 4.6 reveals electrons that are localized or delocalized over the individual atomic cores. The graphic with an ELF value of 0.5 should correspond to a free electron gas (and lower), thus to delocalized charge which is in all cases still centered at individual atoms. The result that the chalcogen cluster itself does not show

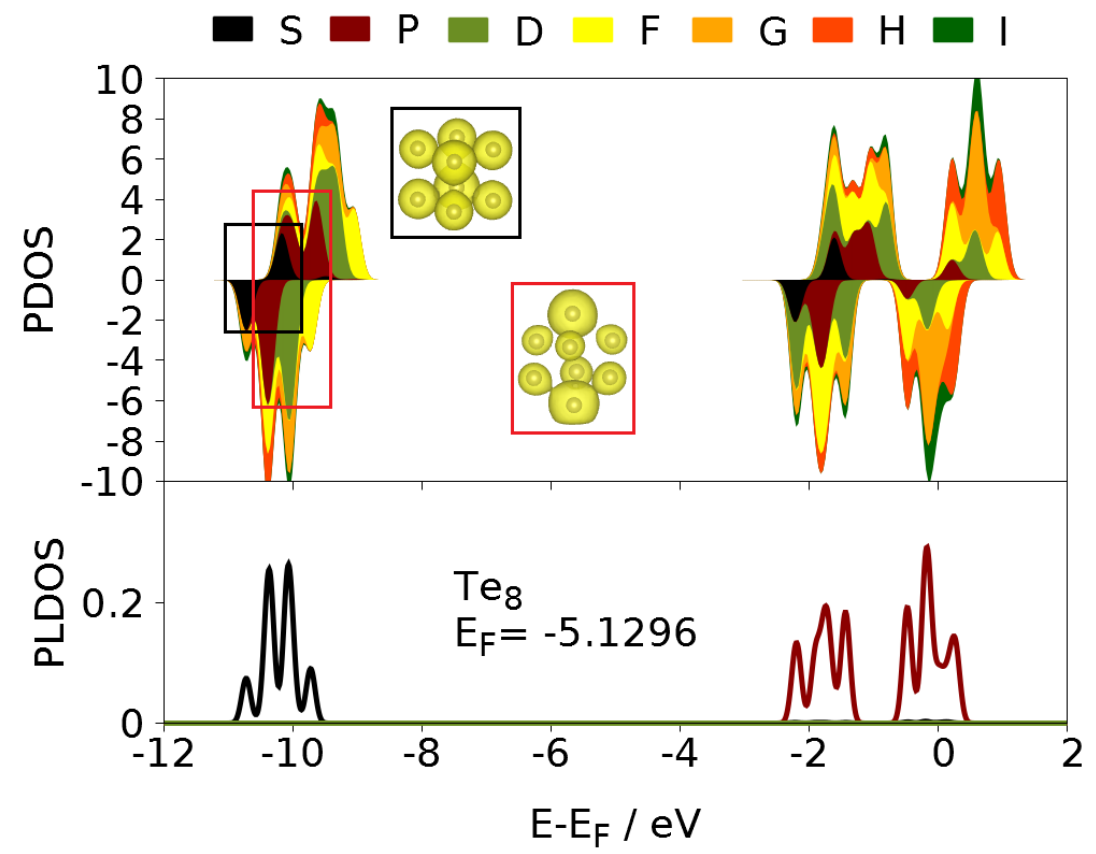

Figure 4.5: Visualization of the PDOS, the PLDOS and selected bands (isosurface cutoff= $0.0008 \mathrm{eV} / \AA)$ for $\mathrm{Te}_{8}$ as taken from the relaxed structure of $\mathrm{Cr}_{6} \mathrm{Te}_{8}\left(\mathrm{PEt}_{3}\right)_{6}$. The orbitals and their corresponding energetic position in the PDOS are marked with colored rectangular.

superatomic character is not surprising when considering that tellurium is a metalloid and therefore reveals more localized $p$ and $s$ electrons then a metal does. In addition, there is a large distance between the chalcogen atoms (3.6-3.8 $\AA$ ) since they encapsulate 


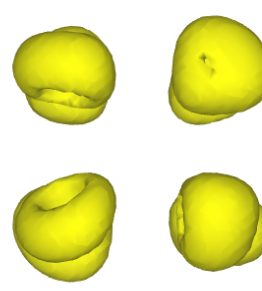

0.7
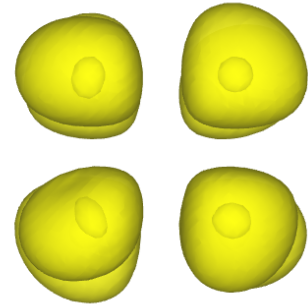

0.5

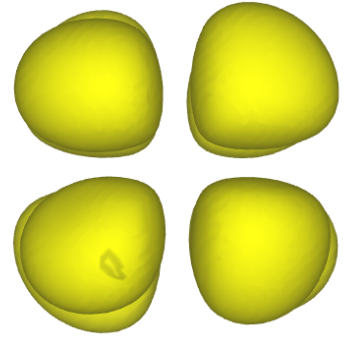

0.3

Figure 4.6: Visualization of the electron localization function for $\mathrm{Te}_{8}$ as taken from the relaxed structure of $\mathrm{Cr}_{6} \mathrm{Te}_{8}\left(\mathrm{PEt}_{3}\right)_{6}$. An ELF value of 1 is usually considered to reflect perfect localization, whereby 0.5 should in theory be equivalent to a uniform electron gas. A value below 0.5 can be associated with a higher Pauli repulsion present then in the free electron gas. $\left[\frac{184,[1 \times 5.5]}{}\right.$

the transition metal cluster.

Similar trends were found when investigating $\mathrm{Te}_{6}$ (fragment of $\left.\mathrm{Ni}_{9} \mathrm{Te}_{6}\left(\mathrm{PEt}_{3}\right)_{8}\right)$ and $\mathrm{Se}_{8}$ (fragment of $\left.\mathrm{Co}_{6} \mathrm{Se}_{8}\left(\mathrm{PEt}_{3}\right)_{6}\right)$. In the case of $\mathrm{Te}_{6}$ the atoms where found to be even further apart (4.2 $\AA$ ). Moreover, selenium is often considered not to be metallic, supporting the observation of a non-superatomic nature.

The second cluster component that could exhibit superatomic character and therefore theoretically make the whole cluster reveal superatomic nature is the transition metal substructure. The fragments corresponding to substructure B (see Fig. 4.4) are an octahedral $\mathrm{Co}_{6}$ and $\mathrm{Cr}_{6}$ cluster and a cubic $\mathrm{Ni}_{9}$ cluster containing a central atom.

Fig. 4.7 shows the angular momentum projected density of states, the individual atomic orbital contributions to the bands, and a visualization of selected superatomic orbitals for the $\mathrm{Co}_{6}$ substructure with 14 unpaired electrons. The PDOS depicts the existence of superatomic S and P states below the Fermi level and a D like LUMO. This is reinforced by the visualization of the corresponding bands that can be found in the same graphic. Integrating over those $\mathrm{S}$ and $\mathrm{P}$ states yields a surface ratio of $1.88: 3.21$ or $1: 1.7(\mathrm{~S}: \mathrm{P})$, indicating that the superatomic structure of the cluster is formed by six delocalized electrons leading to a $\mathrm{S}^{2} \mathrm{P}^{4}$ configuration. This is in good agreement with the assumption that only the $s$ electrons of the transition metals can contribute to the superatomic structure since the pseudopotential used for cobalt relies on a $3 d^{8} 4 s^{1}$ atomic ground state 


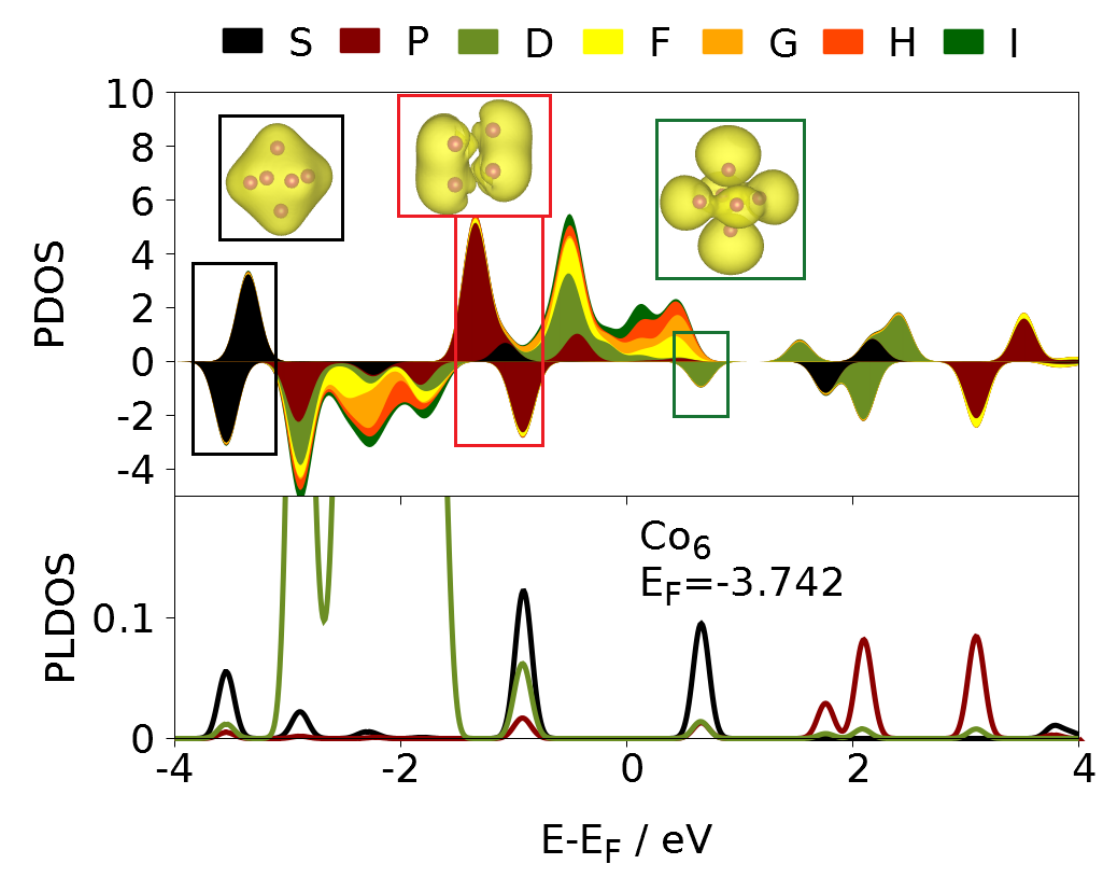

Figure 4.7: Visualization of the angular momentum projected density of states, the individual atomic orbital contributions to the bands, and selected superatomic orbitals for the $\mathrm{Co}_{6}$ substructure as taken from the relaxed structure of $\mathrm{Co}_{6} \mathrm{Se}_{8}\left(\mathrm{PEt}_{3}\right)_{6}$. The orbitals and their corresponding energetic position in the PDOS are marked with colored rectangular. 
configuration. The idea of six superatomic electrons present in this system is further emphasized by an additional P state above the Fermi level and the PLDOS. The latter shows that all occupied S and P states found in the PDOS are mainly contributed to by atomic $s$ electrons.

Fig. 4.8 shows a similar graphic as discussed for the cobalt cluster depicting the PDOS,

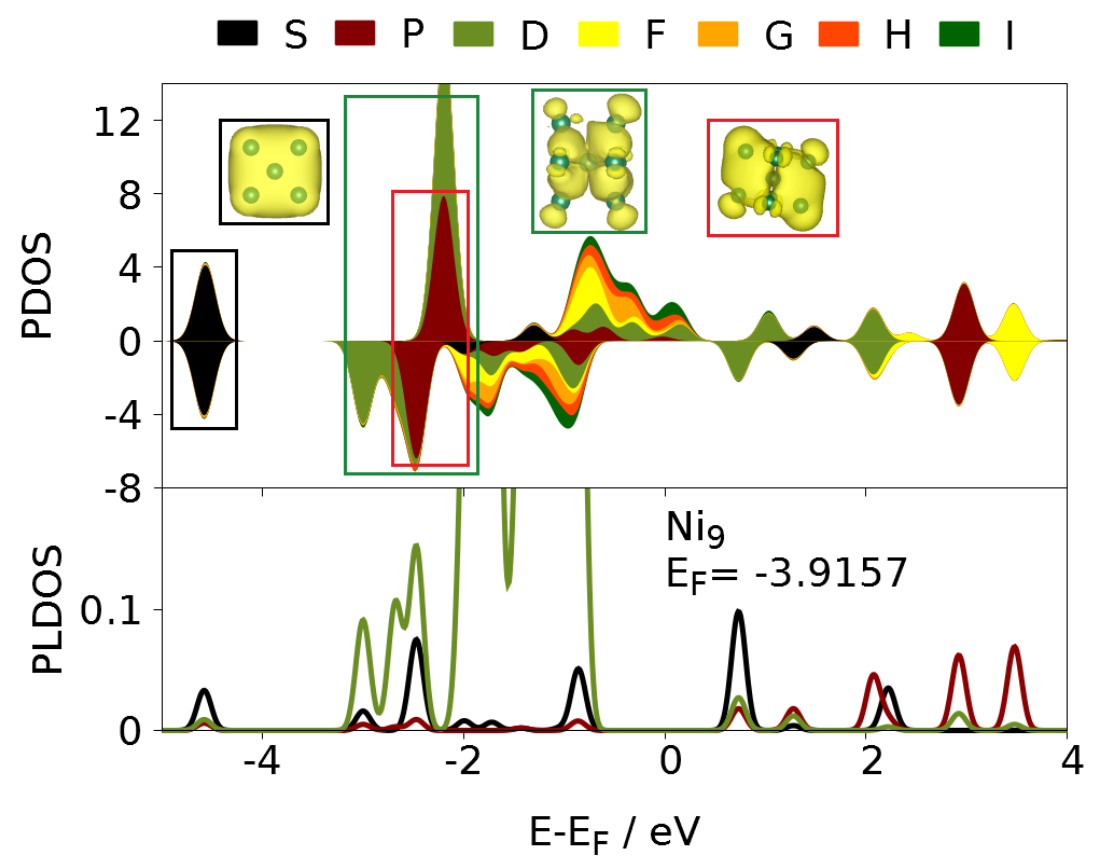

Figure 4.8: Visualization of the angular momentum projected density of states, the individual atomic orbital contributions to the bands, and selected superatomic orbitals for the $\mathrm{Ni}_{9}$ substructure as taken from the relaxed structure of $\mathrm{Ni}_{9} \mathrm{Te}_{6}\left(\mathrm{PEt}_{3}\right)_{8}$. The orbitals and their corresponding energetic position in the PDOS are marked with colored rectangular.

PLDOS and selected superatomic orbitals for the $\mathrm{Ni}_{9}$ cluster revealing eight unpaired electrons. According to the assumption that only the atomic s electrons contribute to the superatomic structure it would be expected to see nine superatomic electrons in the case of a $\mathrm{Ni}_{9}$ cluster leading to a $\mathrm{S}^{2} \mathrm{P}^{6} \mathrm{D}^{1}$ configuration. However, in this case the central atom is complicating the situation. Castleman et al. have shown that the $d$ orbitals of the central transition metal atom can interact with the superatomic electrons in order to stabilize a favored high-spin state ${ }^{[22]}$. Furthermore, those states have found to be potentially lower in energy then superatomic P states. 
The PDOS analysis shows a clear superatomic S state which is confirmed by the visualization of the orbital and the corresponding PLDOS. The P and D configuration however cannot simply be taken from the PDOS graphic. An explanation for this can be found by taking a look at the second orbital visualized in Fig. 4.8. This projection shows that although the PDOS is indicating a superatomic D state at this position, the state is effectively made up by a $d$ orbital of the central atom interacting with the surrounding atoms. Moreover, it can be taken from the PLDOS that $s$ electrons of the surrounding nickel atoms are involved in creating those states. Thus, some of the electrons avail-

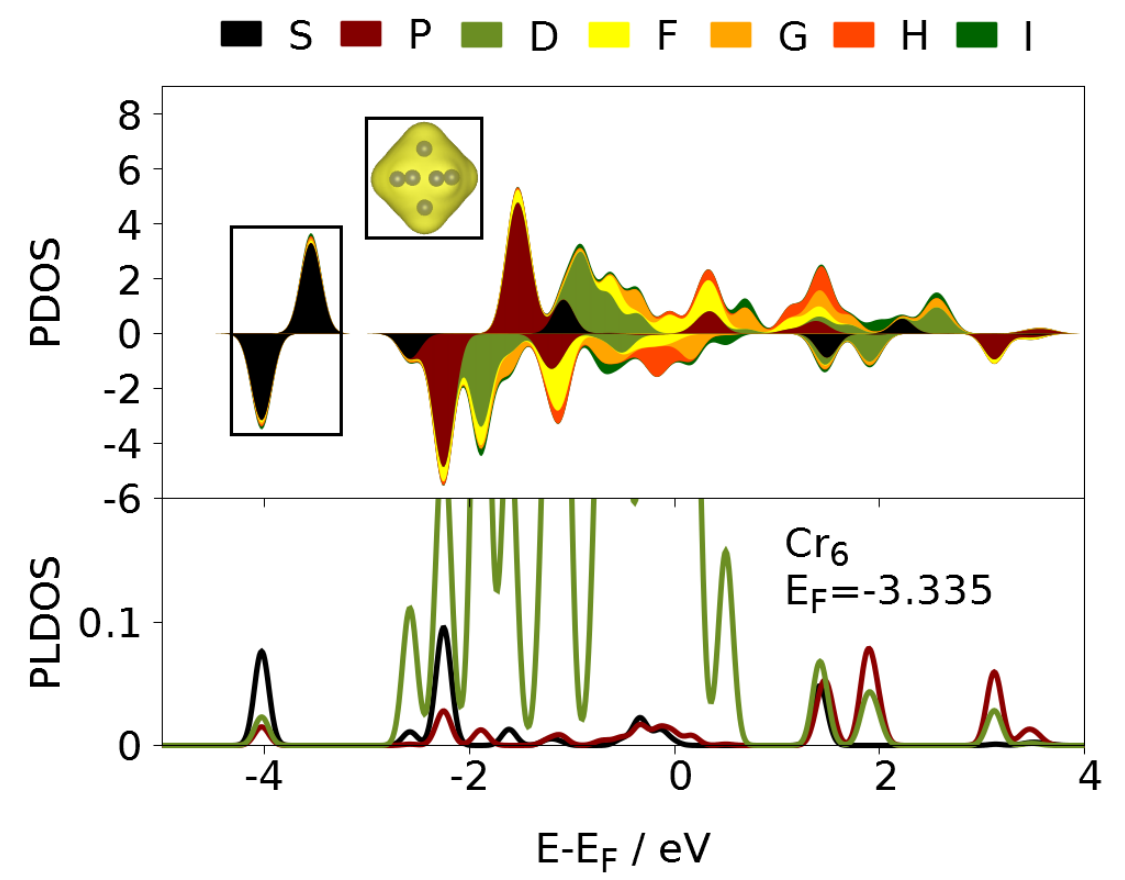

Figure 4.9: Visualization of the angular momentum projected density of states, the individual atomic orbital contributions to the bands, and the superatomic $\mathrm{S}$ orbital for the $\mathrm{Cr}_{6}$ substructure as taken from the relaxed structure of $\mathrm{Cr}_{6} \mathrm{Te}_{6}\left(\mathrm{PEt}_{3}\right)_{6}$. The orbitals and their corresponding energetic position in the PDOS are marked with colored rectangular.

able for the superatomic structure are engaging in creating states with the d orbitals of the central atom. Integrating over the S, P and D states below the Fermi energy as shows the PDOS yields a surface ratio of 2.4:4.7:5.27 or 1:2:2.2 (S:P:D), indicating a $\mathrm{S}^{2} \mathrm{P}^{4}$ configuration for the $\mathrm{S}$ and $\mathrm{P}$ state. Regarding the D state previous investigations 
have revealed that atomic $d$ electrons of the central atom are contributing. Thus, the integration has no significance in this particular case. Furthermore it is questionable if those states can be considered as superatomic. Assuming that the latter is the case would lead to a $\mathrm{S}^{2} \mathrm{P}^{4} \mathrm{D}^{3}$ superatomic configuration.

The electronic situation becomes even more complex in the case of the $\mathrm{Cr}_{6}$ subcluster. Fig. 4.9 depicts the angular momentum projected density of states, the individual atomic orbital contributions to the bands, and a visualization of the superatomic orbitals $\mathrm{S}$ for the $\mathrm{Cr}_{6}$ cluster with 12 unpaired electrons. Already the initial spin state calculation showed a discrepancy between the spin state calculated with PBE (VASP) and the one obtained with the S12g functional (ADF) revealing that PBE is not able to capture the complicated electronic situation present in chromium. This is further emphasized by looking at the complicated structure visualized in the PDOS and the PLDOS. Moreover taking a look at all orbitals, the only orbital that was found to be a clearly visual superatomic state is the $\mathrm{S}$ orbital as shown in Fig. 4.7. Integrating over the occupied S and P states of the PDOS yields a 2.16:3.03 or 1:1.4 (S:P) ratio contradicting the assumption that the six 4 s electrons present in the cluster all contribute to the superatomic electronic structure. In summary all mentioned points indicate that the electronic configuration of chromium is too strongly multi-reference in character to be captured properly with DFT. Nevertheless, from experience with the cobalt and nickel substructures and the visual superatomic S orbital, one would expect the cluster to exhibit superatomic behavior.

The next step is to investigate a combination of the chalcogen cluster with the transition metal cluster yielding substructure $\mathrm{C}$ as visualized in Fig. 4.4 and therefore $\mathrm{Co}_{6} \mathrm{Se}_{8}$, $\mathrm{Cr}_{6} \mathrm{Te}_{8}$ and $\mathrm{Ni}_{9} \mathrm{Te}_{6}$. Table 4.3 is listing the Bader charges of the transition metal atoms

Table 4.3: Bader charges for the transition metal sub structures of the following metalchalcogen clusters: $\mathrm{Co}_{6} \mathrm{Se}_{8}, \mathrm{Cr}_{6} \mathrm{Te}_{8}$ and $\mathrm{Ni}_{9} \mathrm{Te}_{6}$.

\begin{tabular}{cc}
\hline \hline transition metal & Bader charge \\
\hline \hline $\mathrm{Co}$ & 2.90 \\
$\mathrm{Cr}$ & 3.38 \\
$\mathrm{Ni}$ & 1.41 \\
\hline \hline
\end{tabular}

within these clusters. All values indicate a charge transfer from the transition metal 
atoms to the respective chalcogen atoms. The charge transfer is particularly high for the $\mathrm{Co}_{6} \mathrm{Se}_{8}$ and $\mathrm{Cr}_{6} \mathrm{Te}_{8}$ cluster. Considering this information the combination of both

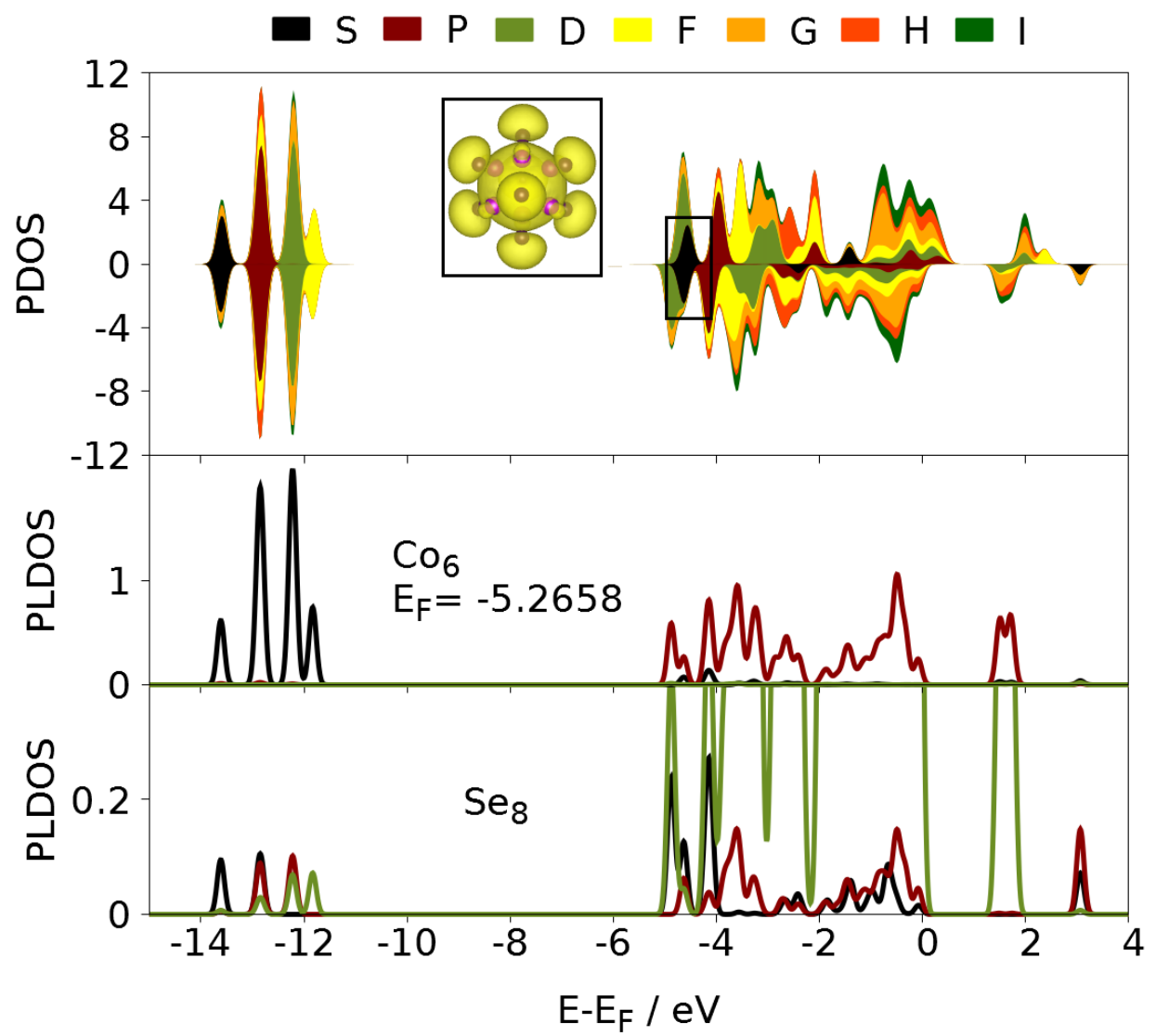

Figure 4.10: Visualization of the angular momentum projected density of states, the individual atomic orbital contributions to the bands for $\mathrm{Co}_{6}$ and $\mathrm{Se}_{8}$ separately, a selected orbital for the $\mathrm{Co}_{6} \mathrm{Se}_{8}$ fragment as taken from the relaxed structure of $\mathrm{Co}_{6} \mathrm{Se}_{8}\left(\mathrm{PEt}_{3}\right)_{6}$. The orbital and the corresponding energetic position in the PDOS are marked with colored rectangular.

substructures might lead to a destruction of the superatomic structure present in the transition metal clusters. The latter can indeed be observed when further investigating the $\mathrm{Co}_{6} \mathrm{Se}_{8}$ cluster. Taking a look at the PDOS and the PLDOS of the Co substructure and the Se cluster separately, as visualized in Fig. 4.10, shows that the s electrons of the Co atoms are now contributing to the low energy states found for the Se cluster. These states were found to be localized or delocalized at the individual Se atoms and contributed to by atomic $s$ electrons. Further verification has been obtained by calculating 
the electron localization function and in addition by visual evaluation of the depicted orbitals. As a consequence of the charge transfer there is not enough delocalized valence electron density left to occupy the superatomic states of the cobalt substructure.

Thus, the only superatomic state found is the $\mathrm{S}$ state, which is visualized in the PDOS. It can be seen that this state is a mixed state that is made up by the transition metal S orbital and the atomic Se $s$ electrons. Both electronic structures are clearly separated again indicating that the Selenium does not contribute to the superatomic nature.

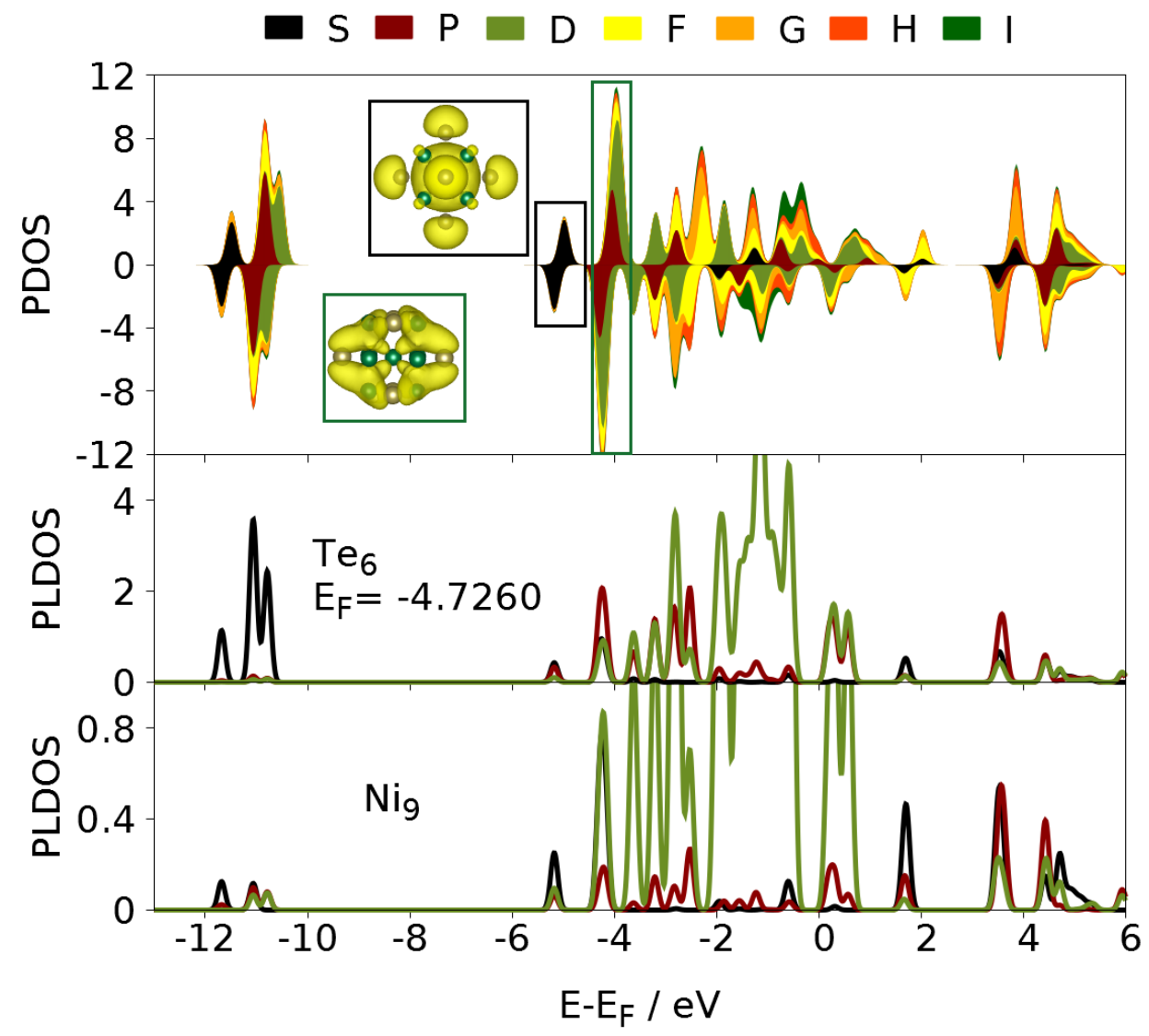

Figure 4.11: Visualization of the angular momentum projected density of states, the individual atomic orbital contributions to the bands for $\mathrm{Ni}_{9}$ and $\mathrm{Te}_{6}$ separately, and selected orbitals for the $\mathrm{Ni}_{9} \mathrm{Te}_{6}$ fragment as taken from the relaxed structure of $\mathrm{Ni}_{9} \mathrm{Te}_{6}\left(\mathrm{PEt}_{3}\right)_{8}$. The orbitals and their corresponding energetic position in the PDOS are marked with colored rectangular.

Since the $\mathrm{Cr}_{6} \mathrm{Te}_{8}$ was found to be problematic due to the insufficiency of DFT in capturing the electronic structure of $\mathrm{Cr}$ properly, $\mathrm{Cr}_{6} \mathrm{Te}_{8}$ will not be discussed in detail. 
However, if the $\mathrm{Cr}_{6}$ substructure should reveal superatomic nature, which is to be expected, adding Te to the system should result in a similar trend as observed in $\mathrm{Co}_{6} \mathrm{Se}_{8}$ since the charge transfer from the transition metal to the chalcogen is even higher in the case of $\mathrm{Cr}_{6} \mathrm{Te}_{8}$.

A similar analysis has been done for the $\mathrm{Ni}_{9} \mathrm{Te}_{6}$ cluster. The corresponding PDOSs and PLDOSs can be found in Fig. 4.1]. In the case of this structure, less charge is transferred to the chalcogen cluster, which leads to a higher delocalized valency located at the Ni subfragment and therefore the possibility of more superatomic orbitals to be occupied. The PLDOS of the Ni cluster reveals two states with dominating Ni $s$ electron contribution. These can be identified as an superatomic S orbital and as discussed earlier, a hybridization of a d orbital of the central atom with the surrounding superatomic D orbital. Reminiscent of the $\mathrm{S}$ orbital of $\mathrm{Co}_{6} \mathrm{Se}_{8}$, both states are mixed states revealing contributions from the surrounding Te atoms, while the superatomic orbital itself remains unaltered. Thus, the nickel containing cluster should in theory reveal the strongest superatomic nature.

In a last step, ligands are added to the system. Those ligands are phosphine ligands that are known to bind with their lonepair. Furthermore phosphine ligands are e.g. from gold cluster investigations, known to not directly contribute to the superatomic electron count ${ }^{[186]}$ as for example thiolate ligands do. Nevertheless they do contribute to the confinement of the electrons. Hence, despite additional ligand binding, the general superatomic structure or non existent superatomic structure observed in $\mathrm{Co}_{6} \mathrm{Se}_{8}, \mathrm{Cr}_{6} \mathrm{Te}_{8}$ and $\mathrm{Ni}_{9} \mathrm{Te}_{6}$ remains unchanged.

The other bulk building block that needs to be considered is the $\mathrm{C}_{60}$. Fullerene-type clusters have already been studied both theoretically and experimentally regarding their potential superatomic nature and have indeed been found to reveal unoccupied super-

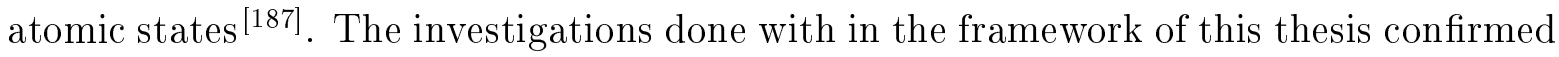
those findings. A visualization of the corresponding PDOS and the superatomic S orbital above the Fermi level can be found in Fig. 4.12. 


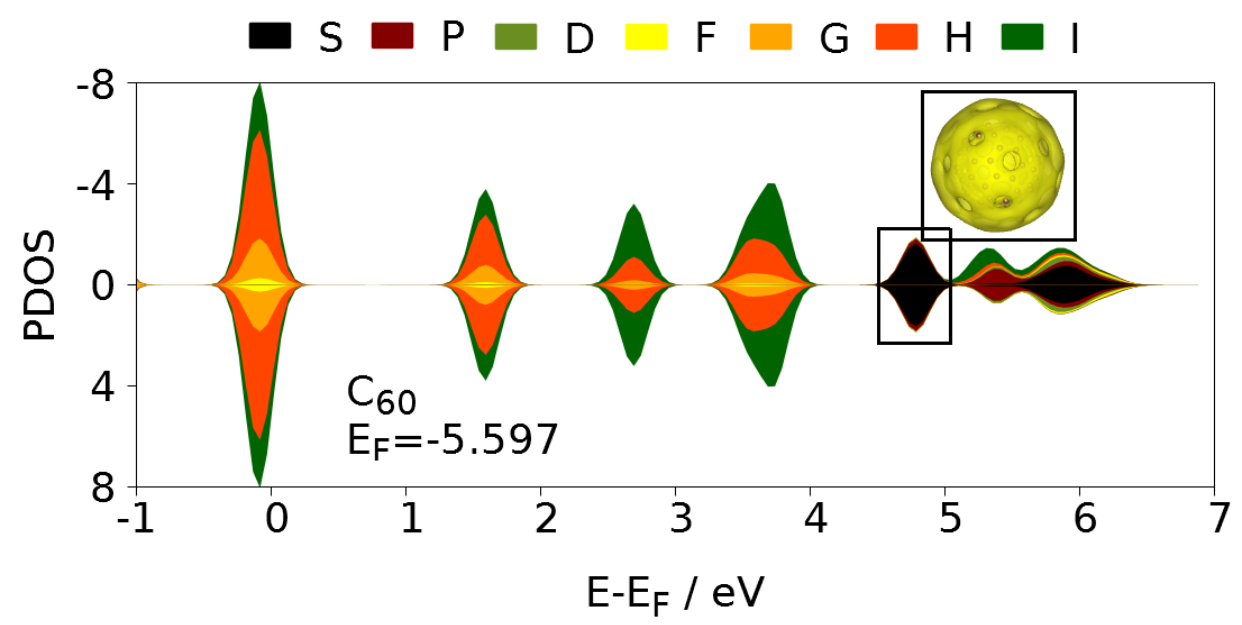

Figure 4.12: Visualization of the angular momentum projected density of states and superatomic $\mathrm{S}$ orbital above the Fermi level for $\mathrm{C}_{60}$.

\subsection{Conclusion}

Starting from a general study of the cluster based compounds synthesized by Nuckolls et al. the investigation has proceeded to an analysis of the superatomic nature of both the compounds and the individual building blocks. The observations done regarding the compounds have shown that the interactions between the building blocks are dominated by van der Waals interactions. However, some contributions to the binding energy that go beyond pure long range interactions, have been identified. Those interactions indicate a behavior reminiscent of atom based solid-state materials. Besides the crystallization of the cluster in atom like manner (rock-salt, $\mathrm{CdI}_{2}$ ), a charge transfer from each cluster to the fullerene has been observed. Moreover, weakly disperse bands have been found in all cases, indicating collective electronic behavior. In addition the interesting magnetic response of the Co and $\mathrm{Cr}$ containing compounds, as predicted by corresponding experiments, has been captured theoretically from the calculated band structures, again indicating collective behavior.

The strongest atom like behavior however was found for $\left[\mathrm{Ni}_{9} \mathrm{Te}_{6}\left(\mathrm{PEt}_{3}\right)_{8}\right]\left[\mathrm{C}_{60}\right]$. The nickel containing compound not only reveals a larger contribution to the cohesive energy when not considering van der Waals interactions, but also shows a hybridization of the $p$ and $d$ orbitals of the cluster and the fullerene, indicating an actual bond. 
In summary, this part of the analysis has shown that the compounds all reveal some atom based bulk behavior which seems to be the strongest in the case of the nickel containing structure. Nevertheless, those bulk materials exhibit many properties that are not similar to their atom based counter parts but more to molecular solids.

To get deeper insight into whether or not those compounds can be called atom-like or superatomic the individual clusters have been investigated to determine the extent of their superatomic nature. It has been found that the chalcogen structure present in the cluster does not contribute electrons that are delocalized over the whole cluster. Moreover, Te and Se seem not to contribute to the superatomic structure found in the Co, $\mathrm{Ni}$ and potentially the $\mathrm{Cr}$ cluster. An observed charge transfer from the transition metal cage to the chalcogen substructure even indicates a partial localization of the transition metal valency in every case. Thus, the presence of the chalcogen leads to an occupation of less superatomic orbitals and furthermore to mixed states. All clusters seem to retain some of their superatomic character whereby it is questionable in how far the mixed state superatomic orbitals can still be regarded as such. Considering the differences between the individual clusters it has been found that the $\mathrm{Ni}_{9} \mathrm{Te}_{6}\left(\mathrm{PEt}_{3}\right)_{8}$ cluster seems to exhibit the strongest superatomic nature due to a smaller charge transfer to the Te thus, more superatomic orbitals being occupied.

The observations made in the case of the individual clusters are in good agreement with the theoretical investigation of the compound. Both indicate some superatomic character which is the strongest in the case of the nickel containing cluster and the corresponding solid-state structure. On the other hand some big deviations away from a perfect superatom and therefore a possible comparison to individual atom based bulk materials arise. Whether or not these materials deserve the label "superatomic" is questionable but as a matter of fact they do reveal some superatomic character.

Beyond this general observation some other details of the overall analysis are worth to be mentioned. The importance of the ligands has been reinforced by showing that they act as a dielectric medium between the cluster and the fullerene, enabling a charge transfer. Moreover, it has been shown in the case of thiolate ligands that additional charges are mainly located at the ligands, which is also the case for these particular clusters, revealing that the ligands engage in creating the superatomic structure. In addition, a good 
agreement with the assumption that only the atomic transition metal $s$ electrons can contribute to the superatomic structure has been achieved, supporting the general idea. It has been shown that these clusters have limited superatomic character. The main problem though when thinking of this particular kind of assemblies is the lack of tunability through doping. Therefore it seems to be reasonable to take what is learned from these solids and predict assemblies made from the previously studied, tunable clusters. 


\section{Screening of Potential Superatomic Compounds}

\subsection{Introduction}

The synthesis of cluster based materials depicts a difficult challenge for experimental chemistry, which is not at least due to a lack of understanding of inter-cluster interactions and the electronic properties of individual clusters. The main advantage of those materials compared to conventional bulk compounds is the "simple" tunability of the chemical and physical characteristics of individual clusters. As a consequence, cluster assemblies can theoretically be used for the creation of potential designer materials exhibiting desired properties. Since superatoms reveal a large intrinsic thermodynamic stability and often very interesting tunable electronic characteristics, they seem to be an excellent choice as building blocks for the bulk.

Superatoms that fulfill all requirements to be regarded as good building blocks for potential materials have been introduced in chapter 3 . As aforementioned ligand protected superatomic gold clusters have an easily tunable electronic structure which, in the case of small clusters, can be strongly altered through the introduction of single atomic impurities. Besides the process of doping the clusters, the change of ligands can also result in a drastic change of the electronic structure mainly caused by the localization of more or fewer electrons. Furthermore, as shown in chapter [3 the electron localization can be tuned by the involvement of the ligands in creating the superatomic states. Other aspects of importance for the consideration of those clusters as building blocks are high stability, and atom like properties such as similar electron affinities.

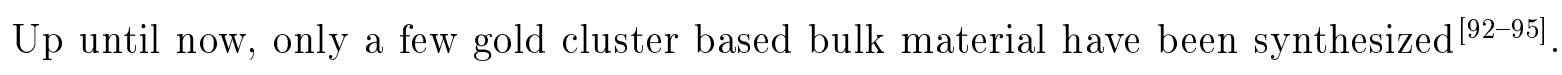


The aim of this chapter is to contribute to the general understanding of those systems and by that to inspire the synthesis of more interesting cluster based materials. In addition, this knowledge about gold based superatoms and their possible compounds is likely transferable to other ligand protected systems with a straightforward superatomic electron count, such as aluminum or sodium clusters.

In this particular part of the thesis, the knowledge about the electronic structure and the tunability of the doped gold clusters from chapter 3 is used, to study dimer interactions between electron donor and acceptor clusters. The goal is to subsequently study potential bulk materials, chosen based on aspects such as the binding energy between superatomic elements, charge transfer and the relative size of the clusters. The underlying structures of the bulks were chosen according to Pauling's simple radius ratio rule for binary ionic structures ${ }^{[1 \times 8]}$. Table 5.0 lists the cation-anion radius ratio and the

Table 5.1: Cation-anion radius ratio and resulting binary solid-state coordination environments according to Pauling.

\begin{tabular}{cccc}
\hline \hline coordination number & structure type & radius ratio & example \\
\hline 4 & tetrahedral & $0.225-0.414$ & $\mathrm{ZnS}$ \\
6 & octahedral & $0.414-0.732$ & $\mathrm{NaCl}$ \\
8 & cubic & $0.732-1.000$ & $\mathrm{CsI}$ \\
\hline \hline
\end{tabular}

proposed coordination environment of each ion in the resulting solid-state structure and examples for these structures that have been used in order to construct the cluster based materials.

\subsection{Methods}

Most of the calculations discussed in the following passages have been conducted using the program package VASP. The generalized gradient approximation PBE ${ }^{[129,[30]}$ was chosen as DFT functional. In most cases the calculation has been repeated using the D3 correction by Grimme et al. ${ }^{[134]}$ for the inclusion of van der Waals interactions. Furthermore, all calculations have been performed spin polarized and without symmetry restrictions. In the case of all dimer and cluster calculations dipole and quadrupole corrections have been included and the energy convergence criterion was set to $10^{-5} \mathrm{eV}$. The 
ionic relaxation was stopped when all forces dropped below $0.02 \mathrm{eV} / \AA$. The Brillouin zone integration was done using Gaussian smearing with a smearing factor of 0.01 in all cases.

In the case of all bulk single point and relaxation calculations the energy convergence criterion was set to $10^{-4} \mathrm{eV}$, while the ionic relaxation was stopped when all forces dropped below $0.02 \mathrm{eV} / \AA$. Due to computational complexity the dispersion corrected $\mathrm{Au}_{12} \mathrm{Al}\left[\mathrm{RS}(\mathrm{AuSR})_{2}\right]_{6}-\mathrm{Au}_{13}\left[\mathrm{RS}(\mathrm{AuSR})_{2}\right]_{6}$ assembly has been converged to $0.03 \mathrm{eV} / \AA$, whereby the dispersion corrected dimer has been converged to a force threshold of $0.02 \mathrm{eV} / \AA$. Moreover the k-grid was chosen to be a $4 \times 4 \times 4 \Gamma$-centered mesh except for the bulk consisting of $\mathrm{Au}_{12} \mathrm{Al}\left[\mathrm{RS}(\mathrm{AuSR})_{2}\right]_{6}$ and $\mathrm{B}_{11} \mathrm{H}_{14}$. In the latter case the grid was chosen to be $2 \times 2 \times 2$, due to the larger size of the primitive cell. Similar to the dimer and cluster calculations, Gaussian smearing with a factor of 0.01 was applied.

The calculation of the band structures and the density of states have been conducted with a decreased the smearing factor (0.005) and increased size of the k-mesh, $4 \times 4 \times 4$ in the case of the borane containing bulk and a $8 \times 8 \times 8 \Gamma$-centered mesh in all other cases. Beyond VASP calculations, additional computation (EDA analysis) was performed using the software package ADF. The results have been obtained using the GGA functional PBE and a TZ basis set including two polarization functions. Relativistic effects have been included using ZORA [ए79-[181] . The fragments chosen for the EDA analysis have been considered to be charged for those materials and dimers with a charge transfer of at least 0.4 e according to Bader charge analysis. Where the Bader charges reveal a smaller charge transfer, the EDA fragments have been chosen to be uncharged.

\subsection{Discussion}

The dimers and bulk materials chosen for investigation are all binary, thus consist of two clusters with one of them being a thiolate protected gold cluster. The main idea is to identify and explain the main inter-cluster interactions, such as van der Waals, ionic or even covalent interactions. Thus, what controls the binding. Chapter B showed that some of the doped gold clusters should theoretically be electron donors while others are electron acceptor superatoms. Furthermore, in the framework of the superatom concept 
the coinage metal doped and the undoped cluster should behave like halogen atoms, while the Pt and Pd doped clusters are more consistent with chalcogen atoms. Moreover, the p-block metal doped clusters can be regarded as electron donor alkali/earth alkali like superatoms. To cover all potential atom like properties, doped gold clusters have been chosen from all divisions and combined with clusters that are known for their electron accepting or donating behavior.

\subsubsection{Superatomic Gold Clusters as Electron Donor Building Blocks}

In order to investigate the electron donating behavior of gold clusters and their interaction with electron accepting building blocks, three different gold clusters have been chosen, namely $\mathrm{Au}_{13}\left[\mathrm{RS}(\mathrm{AuSR})_{2}\right]_{6}, \mathrm{Au}_{12} \mathrm{Al}\left[\mathrm{RS}(\mathrm{AuSR})_{2}\right]_{6}$ and $\mathrm{Au}_{12} \mathrm{Sn}\left[\mathrm{RS}(\mathrm{AuSR})_{2}\right]_{6}$. The nanoparticles have been chosen such that one of them reveals one excess electron above P shell closing and therefore should act as one electron donor. The second cluster could act as a two electron donor and the third is simply the undoped cluster acting as reference system. Due to their, electron accepting behavior and their size, buckyballs and boranes are chosen as acceptor clusters. Furthermore, in the case of the fullerene, a few existing cluster assembled materials are already known (See e.g. chapter [ 7.1$]$ ).

Before the performance of a complete relaxation of the dimer the optimal distance between the two clusters has been estimated by single point calculations at different distances. This procedure has been done for each different acceptor building block. To get a first idea about the interaction between the clusters, the total energy has been plotted as a function of distance and a Lennard-Jones potential based on the calculated potential well was fitted. It was found that the change of energy with distance would be more drastic in the case of individual atoms. This finding is not very surprising, since dispersion interactions between clusters can often be considered as one of the main binding contributions. Thus, this finding only reinforces the previously mentioned idea of dominant long range interactions. Furthermore the shape of the total energy plotted as a function of distance can not be well reproduced by a simple Lennard Jones potential. Table 5.2 shows an overview of the cohesive energies and the Bader charges of the gold 
Table 5.2: Cohesive energies $\mathrm{E}_{\mathrm{coh}}$ and Bader charges of the gold clusters $\mathrm{q}$ calculated for the four different dimers: $\mathrm{Au}_{13}\left[\mathrm{RS}(\mathrm{AuSR})_{2}\right]_{6}-\mathrm{C}_{60}, \mathrm{Au}_{12} \mathrm{Al}\left[\mathrm{RS}(\mathrm{AuSR})_{2}\right]_{6}-\mathrm{C}_{60}$, $\mathrm{Au}_{12} \mathrm{Sn}\left[\mathrm{RS}(\mathrm{AuSR})_{2}\right]_{6}-\mathrm{C}_{60}$ and $\mathrm{Au}_{12} \mathrm{Al}\left[\mathrm{RS}(\mathrm{AuSR})_{2}\right]_{6}-\mathrm{B}_{11} \mathrm{H}_{14}$ using the functional $\mathrm{PBE}$ and $\mathrm{PBE}+$ Grimme's D3 correction.

\begin{tabular}{ccccc}
\hline \hline & \multicolumn{2}{c}{ PBE } & \multicolumn{2}{c}{ PBE+D3 } \\
dimer & Bader charge [q] & $\mathrm{E}_{\text {coh }[\mathrm{eV}]}$ & Bader charge [q] & $\mathrm{E}_{\text {coh }}[\mathrm{eV}]$ \\
\hline $\mathrm{Au}_{13}\left[\mathrm{RS}(\mathrm{AuSR})_{2}\right]_{6}-\mathrm{C}_{60}$ & 0.06 & -0.08 & 0.06 & -0.11 \\
$\mathrm{Au}{ }_{12} \mathrm{Al}\left[\mathrm{RS}(\mathrm{AuSR})_{2}\right]_{6}-\mathrm{C}_{60}$ & 0.25 & -0.05 & 0.26 & -0.11 \\
$\mathrm{Au}_{12} \mathrm{Sn}\left[\mathrm{RS}(\mathrm{AuSR})_{2}\right]_{6}-\mathrm{C}_{60}$ & 0.20 & -0.03 & 0.23 & -0.09 \\
$\mathrm{Au}_{12} \mathrm{Al}\left[\mathrm{RS}(\mathrm{AuSR})_{2}\right]_{6}-\mathrm{B}_{11} \mathrm{H}_{14}$ & 0.92 & -1.71 & 0.88 & -2.47 \\
\hline \hline
\end{tabular}
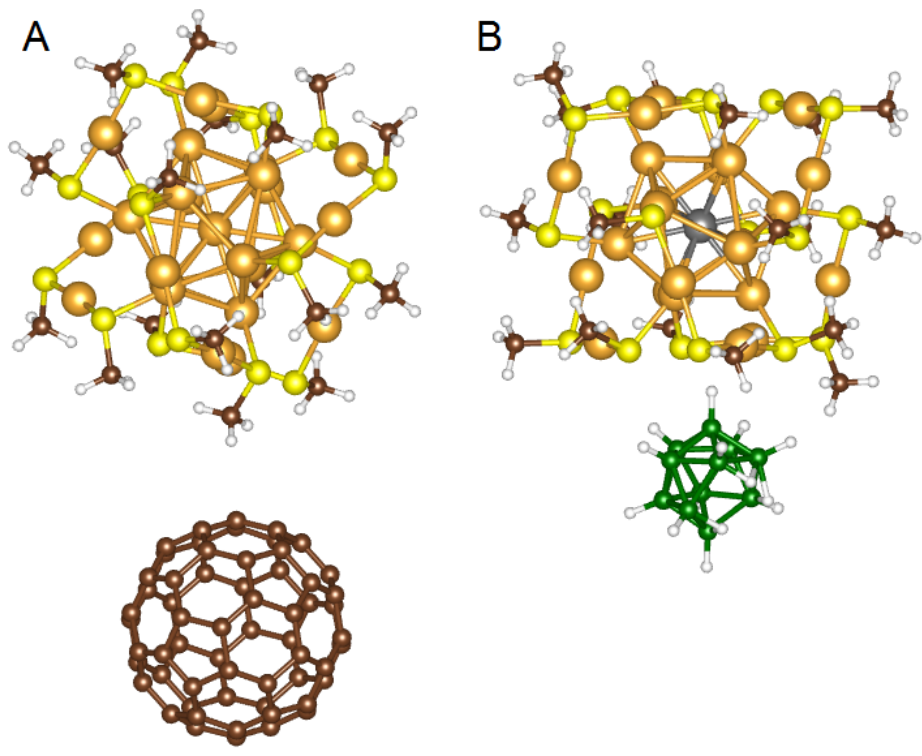

Figure 5.1: Schematic visualization of the two different types of dimers investigated and listed in Table 5.2, A: gold cluster- $\mathrm{C}_{60}$, B: gold cluster- $\mathrm{B}_{11} \mathrm{H}_{14}$. Color code: Au: orange, $\mathrm{S}$ : yellow, C: brown, H: white, B: green, Al: gray. 
cluster obtained for the four different dimers: $\mathrm{Au}_{13}\left[\mathrm{RS}(\mathrm{AuSR})_{2}\right]_{6}-\mathrm{C}_{60}, \mathrm{Au}_{12} \mathrm{Al}\left[\mathrm{RS}(\mathrm{AuSR})_{2}\right]_{6^{-}}$ $\mathrm{C}_{60}, \mathrm{Au}_{12} \mathrm{Sn}\left[\mathrm{RS}(\mathrm{AuSR})_{2}\right]_{6}-\mathrm{C}_{60}$ and $\mathrm{Au}_{12} \mathrm{Al}\left[\mathrm{RS}(\mathrm{AuSR})_{2}\right]_{6}-\mathrm{B}_{11} \mathrm{H}_{14}$ (See Fig. 5.d). It can be seen that in all three fullerene containing compounds low binding energies are obtained. Even considering long range interactions results in cohesive energies of -0.09$-0.11 \mathrm{eV}$ only. The distances found between the clusters are in good agreement with the low binding energies. The closest carbon-carbon atom distance between cluster and fullerene was found to be around 3.4-3.5 $\AA$. The highest binding energy can be observed for the undoped gold cluster containing dimer where the carbon-carbon distance to the fullerene is slightly smaller then in the other two cases (distance range: 0.0005-0.08 $\AA$ ) enabling slightly better interaction. Furthermore the Bader charges indicate no charge transfer in the case of the undoped cluster, while a relatively small charge transfer can be observed in both other cases.

From Table [3.3 it can be seen, that the Al doped cluster has a first ionization potential of $4.61 \mathrm{eV}$, while the Sn doped cluster reveals an IP of $4.69 \mathrm{eV}$ and the undoped cluster of $4.93 \mathrm{eV}$. This is in good agreement with the observed Bader charge values, which indicate the smallest or no charge transfer occurring in the dimer containing the undoped cluster. Moreover, the rather small charge transfer observed in the case of the one and two electron donor clusters can be explained by a relatively small EA value for the fullerene, namely $-2.83 \mathrm{eV}$.

Due to its lower IP value and the higher binding energy in the fullerene containing dimer, $\mathrm{Au}_{12} \mathrm{Al}\left[\mathrm{RS}(\mathrm{AuSR})_{2}\right]_{6}$ has been chosen to also be investigated in a dimer containing a $\mathrm{B}_{11} \mathrm{H}_{14}$ nido borane (See Fig. 5.7). In contrast to the fullerene containing dimers, a strong charge transfer and a relatively large binding energy can be observed regarding the $\mathrm{Au}_{12} \mathrm{Al}\left[\mathrm{RS}(\mathrm{AuSR})_{2}\right]_{6}-\mathrm{B}_{11} \mathrm{H}_{14}$ dimer. Fig. 5.7 gives a general idea of the structure present in both types of dimers. It can be seen, that the borane exhibits a sufficiently small size for the ligands to move away, such that the borane can approach the cluster core. The small distance between both particles increases the orbital overlap and the attractive electrostatic interaction (see section 5.3.4). Even though the distance further decreases with the inclusion of dispersion interactions and the attractive binding energy increases, less charge transfer can be observed in this particular case. However, this might indicate a weakness of the Bader charge approach e.g. the definition of the Bader 
volumes. Nevertheless it can be generally stated that a strong charge transfer can be observed in the $\mathrm{Au}_{12} \mathrm{Al}\left[\mathrm{RS}(\mathrm{AuSR})_{2}\right]_{6}-\mathrm{B}_{11} \mathrm{H}_{14}$ dimer which can be explained by the high first electron affinity of $8.21 \mathrm{eV}$ computed for the borane. Beyond that the $\mathrm{C}_{60}$ as well as the borane are superatoms, whereby the $\mathrm{C}_{60}$ only reveals unoccupied superatomic orbitals that can be found at high energy ${ }^{[187]}$. Thus, the borane exhibits a stronger superatomic character and is therefore a more suitable choice for creating stable superatomic compounds (See Fig. 5.2). Subsequent to the dimer investigation $\mathrm{Au}_{12} \mathrm{Al}\left[\mathrm{RS}(\mathrm{AuSR})_{2}\right]_{6}-\mathrm{C}_{60}$

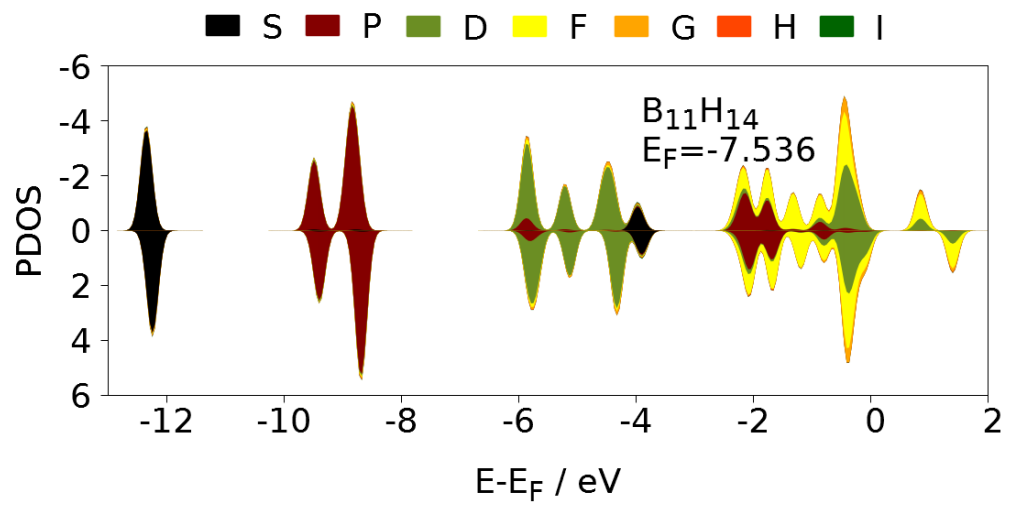

Figure 5.2: Visualization of the PDOS of $\mathrm{B}_{11} \mathrm{H}_{14}$.

and $\mathrm{Au}_{12} \mathrm{Al}\left[\mathrm{RS}(\mathrm{AuSR})_{2}\right]_{6}-\mathrm{B}_{11} \mathrm{H}_{14}$ have been chosen to be investigated in the potential binary bulk assembly. Based on the relative cluster sizes (see Table 5. 1) the fullerene based solid-state material has been constructed in a rock-salt type structure whereas the borane containing solid was set up in a zincblende structure. Both solids were fully relaxed.

\subsubsection{1 $\mathrm{Au}_{12} \mathrm{Al}\left[\mathrm{RS}(\mathrm{AuSR})_{2}\right]_{6}-\mathrm{C}_{60}$ Assembly}

Figure 5.3 visualizes the bulk structure of $\mathrm{Au}_{12} \mathrm{Al}\left[\mathrm{RS}(\mathrm{AuSR})_{2}\right]_{6}-\mathrm{C}_{60}$ relaxed from an initial rock-salt type structure while Table 5.3 summarizes the Bader charge of the gold cluster, the cohesive energy and the lattice parameters calculated for this compound. Based on the low binding contributions obtained for the dimer calculation one could have concluded that both clusters in combination are not a suitable choice as building blocks for bulk materials. 


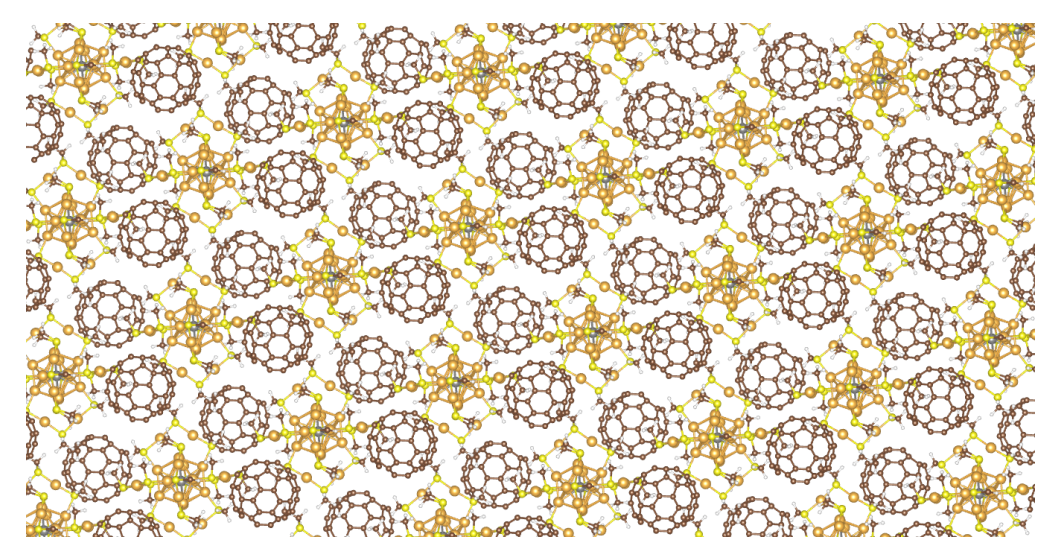

Figure 5.3: Visualization of $\mathrm{Au}_{12} \mathrm{Al}\left[\mathrm{RS}(\mathrm{AuSR})_{2}\right]_{6}-\mathrm{C}_{60}$ in a relaxed rock-salt like bulk structure (PBE).

However, the values listed in Table 5.3 reveal a high cohesive energy present in the binary assembly and a strong charge transfer to the fullerene. In the case of this cluster based solid, a slightly higher charge transfer can be found for the dispersion corrected calculation due to even shorter distances caused by long range interactions between the ligands and the fullerenes. In addition, the inclusion of dispersion is strongly contributing to the binding energy. The PBE obtained cohesive energy (without D3 correction) can already be considered a strong interaction, much higher than in the case of the transition metal cluster based compounds discussed in chapter $\mathbf{\theta}$.

Table 5.3: Cohesive energies $\mathrm{E}_{\mathrm{coh}}$ and Bader charges of the gold clusters $\mathrm{q}$ for $\mathrm{Au}_{12} \mathrm{Al}\left[\mathrm{RS}(\mathrm{AuSR})_{2}\right]_{6} \mathrm{C}_{60}$ in a relaxed rock-salt like bulk structure.

\begin{tabular}{|c|c|c|c|c|c|c|}
\hline & \multicolumn{3}{|c|}{ PBE } & \multicolumn{3}{|c|}{$\mathrm{PBE}+\mathrm{D} 3$} \\
\hline Bader charge [q] & & 0.41 & & & 0.48 & \\
\hline $\mathrm{E}_{\mathrm{coh}}[\mathrm{eV}]$ & & -3.15 & & & -8.11 & \\
\hline lattice parameters $[\AA]$ & $\begin{array}{c}\mathrm{a} \\
15.61\end{array}$ & $\begin{array}{c}\mathrm{b} \\
16.57\end{array}$ & $\begin{array}{c}\mathrm{C} \\
16.94\end{array}$ & $\begin{array}{c}\mathrm{a} \\
14.97\end{array}$ & $\begin{array}{c}\mathrm{b} \\
15.13\end{array}$ & $\begin{array}{c}\mathrm{C} \\
13.94\end{array}$ \\
\hline
\end{tabular}

The underlying situation is somewhat similar to sodium chloride, where the energy needed to ionize the electron donor is larger then the electron affinity of the electron acceptor cluster. This problem is overcome by a high lattice energy of the compound. In the gas phase $\mathrm{NaCl}$ exists as a molecule, thus with a relatively large orbital contribution to binding. Reminiscent of this situation, the energy decomposition analysis presented in 
subsection 5.3.4 has shown, that in the case of the dimer the only attractive contribution to binding is found to be the orbital interaction while in the solid state-material the attractive electrostatic interaction dominates. However, in the dimer both clusters are too far away from each other for any significant interaction to occur. This changes in the assembled bulk material, where shorter distances and high lattice energy lead to a high binding energy and charge transfer to the $\mathrm{C}_{60}$.

Due to the high van der Waals character of the superatomic cluster dimer bond another analogy to individual atoms can be drawn. The binding situation is somewhat similar to the remarkable cases of $\mathrm{Zn}, \mathrm{Cd}$ and $\mathrm{Hg}$, where dimers and small cluster are mostly held together by van der Waals forces ${ }^{[180]}$ similar to noble gas clusters. This significantly changes in the case of the corresponding bulk structures. The solid-state materials consisting of $\mathrm{Zn}, \mathrm{Cd}$ and $\mathrm{Hg}$ are of metallic character and reveal a rather large change in bond distance. In the case of e.g. mercury the band varies from $3.7 \AA$ for the dimer to $3.0 \AA$ for the bulk nearest neighbor distance ${ }^{[170,190]}$. The observed shortening in the binding distance is even more significant in the case of the $\mathrm{Au}_{12} \mathrm{Al}\left[\mathrm{RS}(\mathrm{AuSR})_{2}\right]_{6}-\mathrm{C}_{60}$ superatomic case. Furthermore the calculated superatomic band structure visualized in Fig. 5.4 and a high orbital contribution to the binding situation in the bulk, which will be discussed in subsection 5.3.4, reveal electronic properties emerging from the assembled material.

Beyond that relaxed lattice structure was also found to reveal quite high similarity to a rock salt structure for both cases, considering and not including dispersion interactions (See Fig. 5.3). The lattice parameters of the primitive cell are listed in Table 5.3. In a perfect crystal those values should all be equal. The deviations however lie between 6 and $8.5 \%$, which might be due to distortions e.g. Jahn Teller distortions arising in the cluster (See chapter [3) or simply because the cluster is slightly aspherical.

To further understand the superatomic character of this assembly, an analysis of the electronic structure is needed. Fig. 5.4 visualizes the band structure obtained from the PBE calculation for the minority and majority spin. Disperse bands can be found close to the Fermi level arising from a hybridization of the $\mathrm{C}_{60}$ and gold cluster orbitals. Thus, this observation is in good agreement with the calculated cohesive energy, revealing strong binding contributions beyond van der Waals forces. In addition, to the disperse 


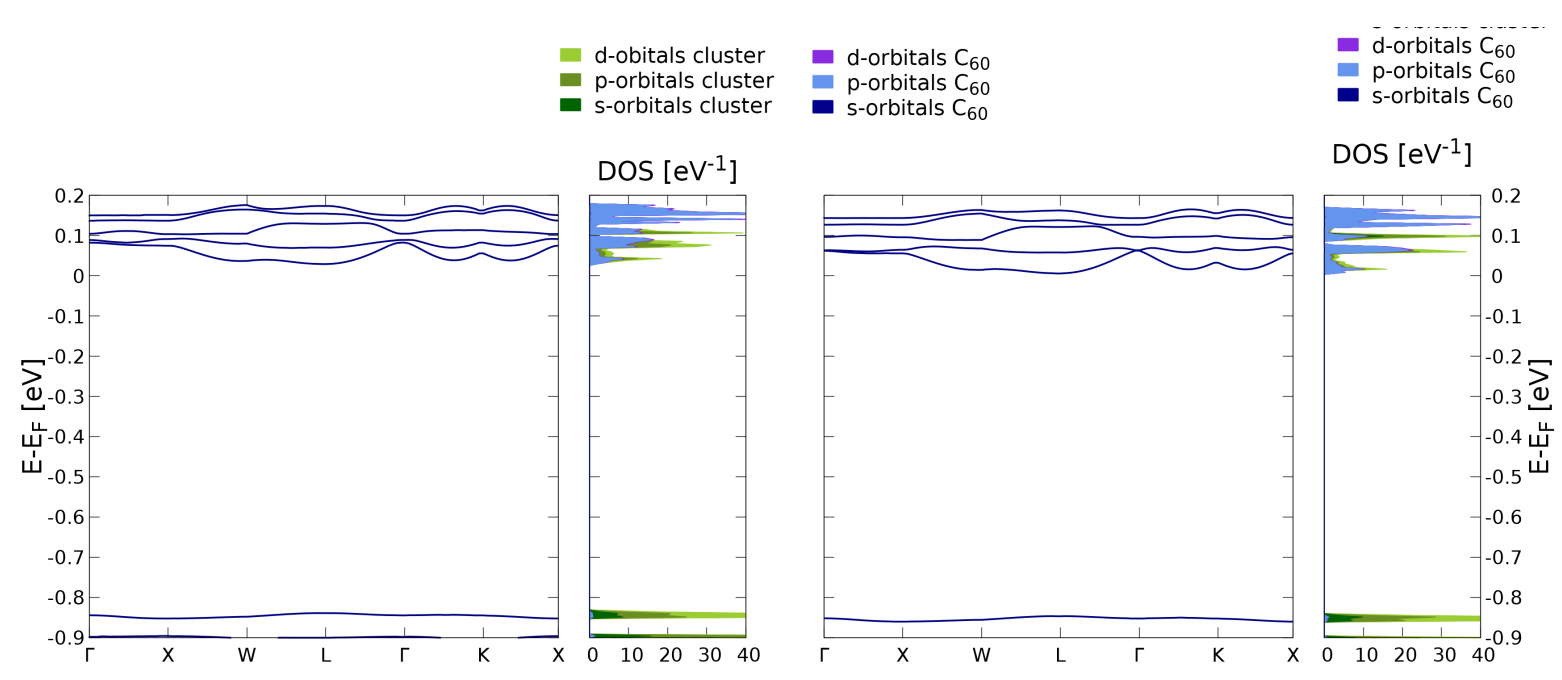

Figure 5.4: Visualization of the band structure and density of states of the rock-salt like $\mathrm{C}_{60^{-}}$ $\mathrm{Au}_{12} \mathrm{Al}\left[\mathrm{RS}(\mathrm{AuSR})_{2}\right]_{6}$ assembly for the majority and minority spin. Calculated along a rock-salt high symmetry path.

bands the DOS reveals, that the HOMO state is mostly centered at the cation of the compound. This indicates a rather small ionic character since the bands in a perfect ionic crystal are flat and the HOMO state is centered at the anion of the material. The band gap of this material is found to be around $910 \mathrm{meV}$, thus in the range of a semiconductor.

For further verification of the superatomic character of this compound the PDOS in a sphere around the gold cluster core has been calculated and visualized in Fig. 5.5. The plot depicts that the electrons that are transferred to the fullerenes come from the superatomic charge of the doped gold cluster. This leads to the stable electronic configuration of $\mathrm{S}$ and $\mathrm{P}$ shell closing in the case of $\mathrm{Au}_{12} \mathrm{Al}\left[\mathrm{RS}(\mathrm{AuSR})_{2}\right]_{6}$.

\subsubsection{2 $\mathrm{Au}_{12} \mathrm{Al}\left[\mathrm{RS}(\mathrm{AuSR})_{2}\right]_{6}-\mathrm{B}_{11} \mathrm{H}_{14}$ Assembly}

Based on the strong interactions present in the borane-Al doped gold cluster dimer, both have also been calculated in a binary assembly. Due to the even smaller size of the acceptor cluster a zincblende type structure was chosen as initial input structure. Fig. 5.6 visualizes the structure of the bulk obtained after relaxation. The structure reveals high similarity to a zincblende type structure. An overview of the charge transfer calculated 


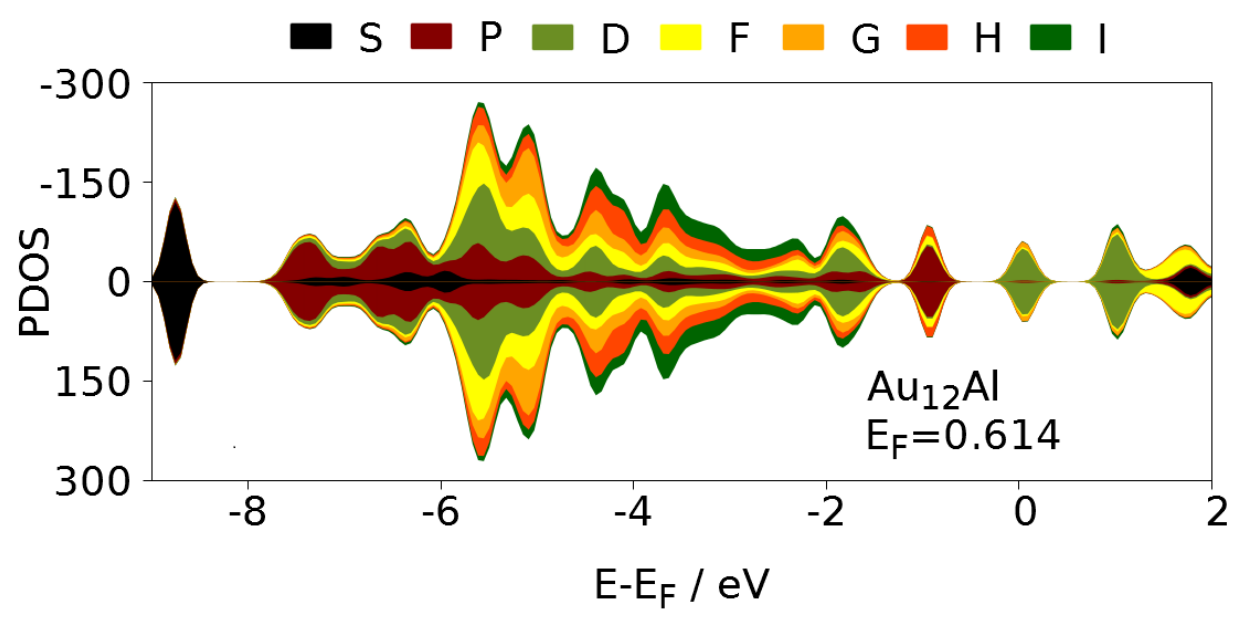

Figure 5.5: Visualization of the PDOS in a sphere around the cluster core of $\mathrm{Au}_{12} \mathrm{Al}\left[\mathrm{RS}(\mathrm{AuSR})_{2}\right]_{6}$ in structure 5.3 .

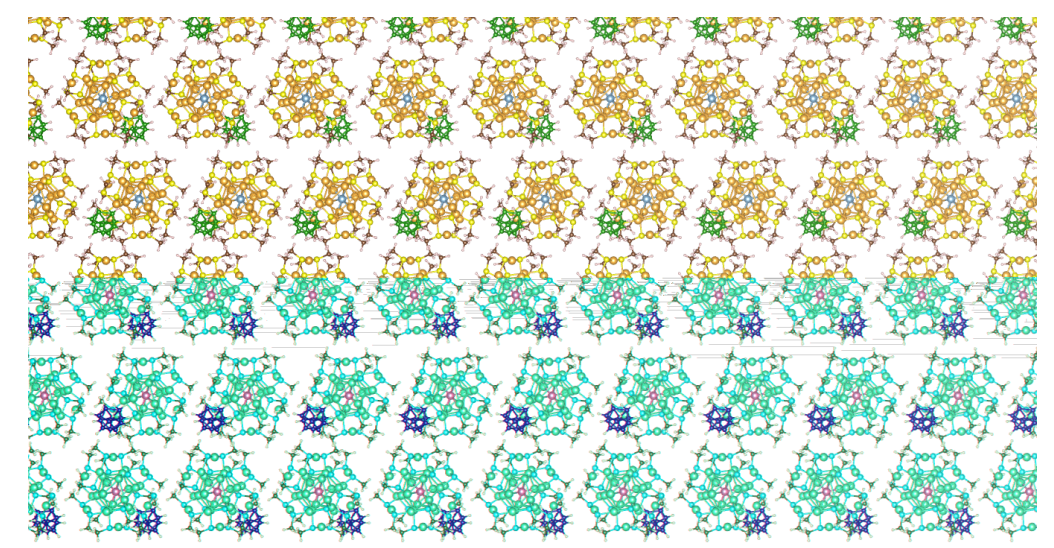

Figure 5.6: Visualization of $\mathrm{Au}_{12} \mathrm{Al}\left[\mathrm{RS}(\mathrm{AuSR})_{2}\right]_{6}-\mathrm{B}_{11} \mathrm{H}_{14}$ in a relaxed zincblende like bulk structure (PBE). 
using the Bader approach, the cohesive energies and the lattice parameters calculated using the functional PBE and PBE $+\mathrm{D} 3$ can be found in Table 5.4. The values in Table 5.4 show on one hand that the lattice parameters $a$ and $b$ remain quite similar to a zincblende structure and that the PBE calculated binding energy is higher than in the case of the $\mathrm{Au}_{12} \mathrm{Al}\left[\mathrm{RS}(\mathrm{AuSR})_{2}\right]_{6}-\mathrm{C}_{60}$ assembly. On the other hand comparing the D3 corrected calculations of the $\mathrm{C}_{60}-\mathrm{Au}_{12} \mathrm{Al}\left[\mathrm{RS}(\mathrm{AuSR})_{2}\right]_{6}$ solid with the borane containing species show a much larger binding energy in the case of $\mathrm{C}_{60}-\mathrm{Au}_{12} \mathrm{Al}\left[\mathrm{RS}(\mathrm{AuSR})_{2}\right]_{6}$. This

Table 5.4: Cohesive energies $\mathrm{E}_{\mathrm{coh}}$ and Bader charges of the gold clusters $\mathrm{q}$ for $\mathrm{Au}_{12} \mathrm{Al}\left[\mathrm{RS}(\mathrm{AuSR})_{2}\right]_{6}-\mathrm{B}_{11} \mathrm{H}_{14}$ in a relaxed zincblende like bulk structure. The cohesive energies have been calculated as the difference between the total energy of the bulk and the individually relaxed components. In this case the obtained energy has been divided by two since each primitive cell includes two gold clusters and two boranes.

\begin{tabular}{ccccccc}
\hline \hline & & PBE & & \multicolumn{3}{c}{ PBE+D3 } \\
Bader charge $[\mathrm{q}]$ & & -0.87 & & & -0.78 & \\
$\mathrm{E}_{\text {coh }}[\mathrm{eV}]$ & & -3.33 & & & -5.99 & \\
\hline lattice parameters $[\AA]$ & $\mathrm{a}$ & $\mathrm{b}$ & $\mathrm{c}$ & $\mathrm{a}$ & $\mathrm{b}$ & $\mathrm{c}$ \\
& 16.41 & 16.19 & 26.78 & 15.10 & 14.33 & 24.97 \\
\hline \hline
\end{tabular}

can be explained by a stronger dispersive contribution in the case of the fullerene gold cluster- than in the case of the borane gold cluster interaction. A reason for this difference in dispersion could be the lower coordination numbers in the case of the $\mathrm{ZnS}$ type structure or simply the nature of the regarded clusters. Moreover, it is noticeable that the Bader charge as well as the bond distance decrease with the inclusion of dispersion interactions. A decrease of bond distance is often accompanied by an increase of charge transfer. This might indicate an insufficiency of the Bader approach in this particular case.

A analysis of the electronic structure similar to the previously discussed bulk structure has been conducted. Figure 5.7 visualizes the PDOS in a sphere around the aluminum doped gold cluster in the solid crystal, again revealing a closed P shell, thus that the transferred electrons are coming from the superatomic charge of cluster $\mathrm{Au}_{12} \mathrm{Al}\left[\mathrm{RS}(\mathrm{AuSR})_{2}\right]_{6}$.

In addition, to the PDOS analysis the band structure has been calculated and visual- 


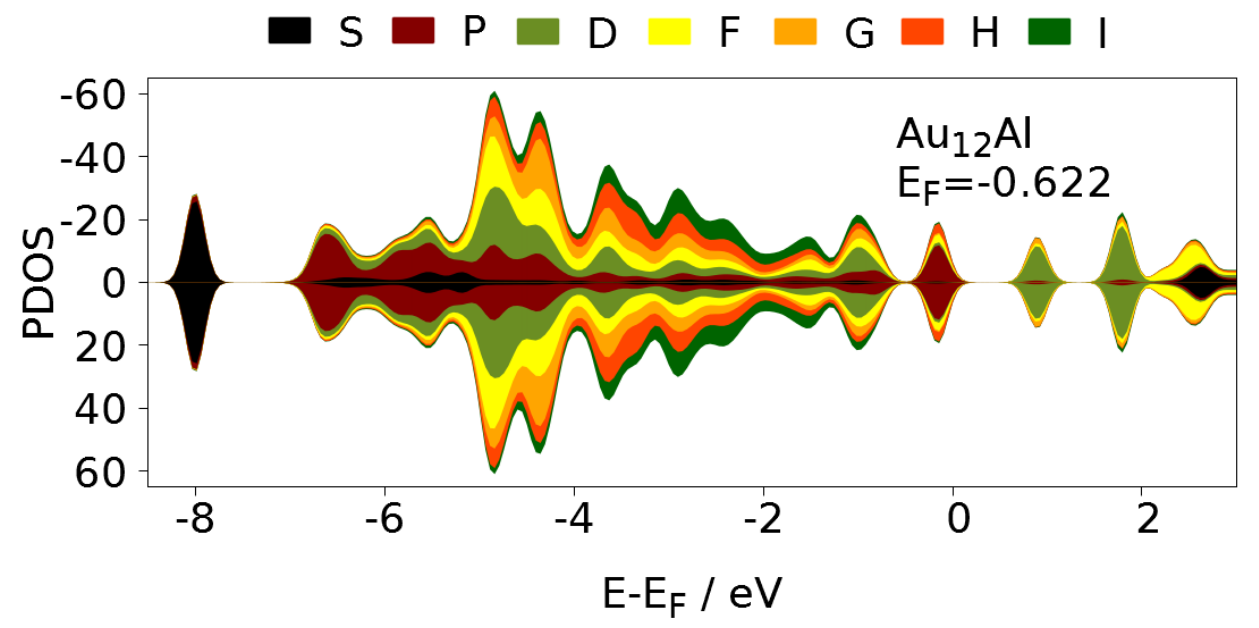

Figure 5.7: Visualization of the PDOS in a sphere around the cluster core of $\mathrm{Au}_{12} \mathrm{Al}\left[\mathrm{RS}(\mathrm{AuSR})_{2}\right]_{6}$ in structure 5.6$]$.

ized in Fig. 5.8. In contrast to the previous case of the fullerene compound relatively flat bands are obtained for the zincblende type bulk. A hybridization of the states as found in the $\mathrm{C}_{60}-\mathrm{Au}_{12} \mathrm{Al}\left[\mathrm{RS}(\mathrm{AuSR})_{2}\right]_{6}$ solid leading to disperse bands, can not be found. Nevertheless, it can be seen that the borane states contribute to a small amount to the depicted gold states. The flat bands indicate a ionic character of the compound than in the previous solid and therefore similarity to $\mathrm{ZnS}$. However, the HOMO state is mostly located at the $\mathrm{Au}_{12} \mathrm{Al}\left[\mathrm{RS}(\mathrm{AuSR})_{2}\right]_{6}$ cluster (the cation) which indicates that the character of the compound is less straightforwardly ionic. In normal ionic solid the HOMO state is found to be located at the anion of the material.

In summary both calculated solid state materials show that there is no direct relationship between the binding energy and the dispersion of the bands. Even though the borane containing solid shows a higher PBE obtained binding energy than the $\mathrm{Au}_{12} \mathrm{Al}\left[\mathrm{RS}(\mathrm{AuSR})_{2}\right]_{6}-\mathrm{C}_{60}$ assembly, less disperse bands have been computed. Therefore the different interactions present in the solid-state materials need to be analyzed in detail. 


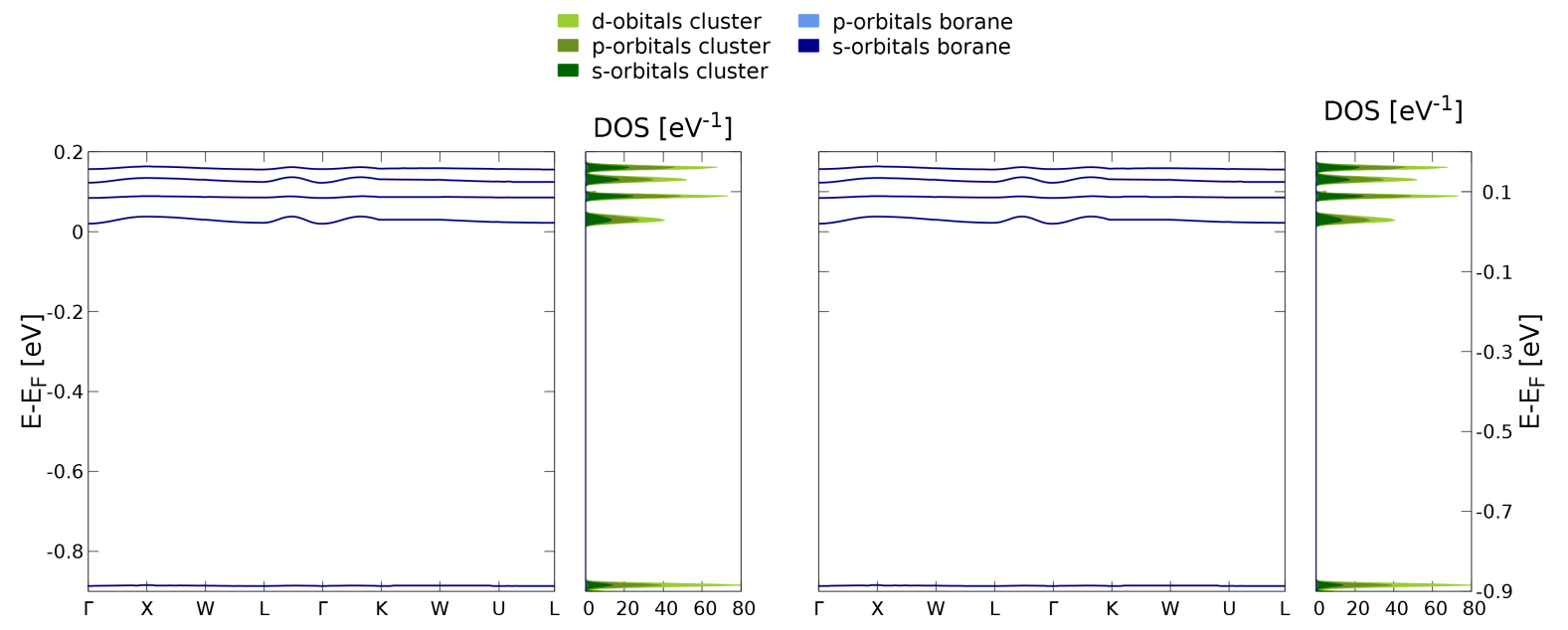

Figure 5.8: Visualization of the band structure and density of states of the zincblende like $\mathrm{B}_{11} \mathrm{H}_{14}-\mathrm{Au}_{12} \mathrm{Al}\left[\mathrm{RS}(\mathrm{AuSR})_{2}\right]_{6}$ assembly for the majority and minority spin. Calculated along a zincblende high symmetry path.

\subsubsection{Superatomic Gold Clusters as Electron Acceptor Building Blocks}

From chapter 3 it was found that the doped gold clusters can not only be used as electron donor building blocks, but that the coinage metal doped clusters have electronic similarity to individual halogen atoms and can therefore act as electron acceptors. To study the electron accepting behavior of the gold cluster an appropriate electron donor is needed. For this a cluster consisting of a $\mathrm{Si}_{16}$ cage encapsulating a Tantalum atom has been calculated. The $\mathrm{Si}_{16} \mathrm{Ta}$ cluster has been synthesized and experimentally investigated by Nakajima et al. [TII] and was claimed to be a stable alkali like superatom with the possibility of being used as a bulk building block [197]. Fig. 5.9 shows the PDOS around the cluster $\mathrm{Si}_{16} \mathrm{Ta}$, revealing that it is indeed a superatomic cluster.

Based on this idea the dimer interaction between both clusters has been calculated (visualized in Fig. 5.T0. A). The binding energy of the dimer has been computed to be $-0.36 \mathrm{eV}$ (not considering dispersion interactions). Even though this value is larger than the binding energy obtained for the gold cluster-fullerene dimers, the expected charge transfer from the Si cluster to the undoped gold cluster has not been observed. In fact a Bader charge of 0.33 suggests that instead a charge transfer is occurring from the gold 


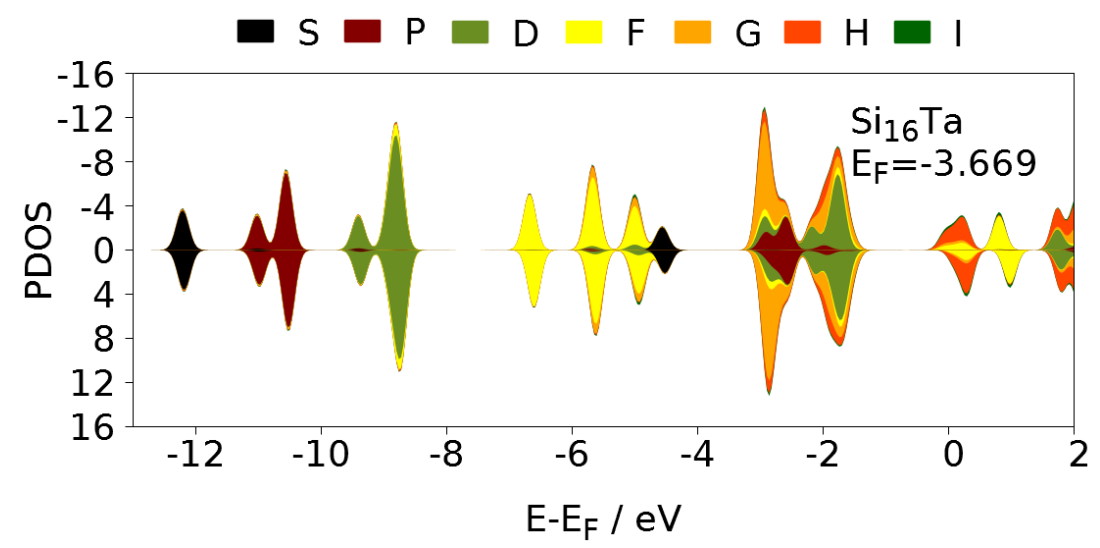

Figure 5.9: Visualization of the angular momentum projected density of states in a sphere around $\mathrm{Si}_{16}$ Ta cluster in vacuum.
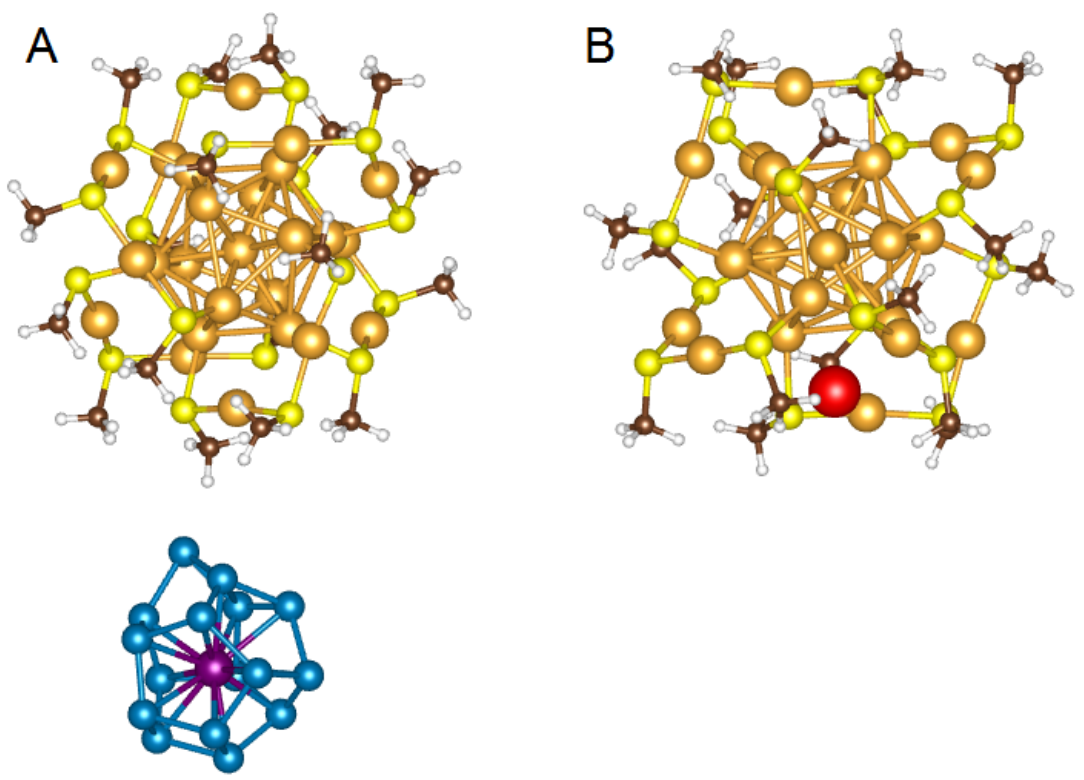

Figure 5.10: Visualization of the relaxed dimers A: $\mathrm{Au}_{13}\left[\mathrm{RS}(\mathrm{AuSR})_{2}\right]_{6}-\mathrm{Si}_{16} \mathrm{Ta}$ and B: $\mathrm{Au}_{13}\left[\mathrm{RS}(\mathrm{AuSR})_{2}\right]_{6}-\mathrm{Na}$. Color code: Au: orange, S: yellow, C: brown, H: white, B: green, Si: blue, Ta: violet and Na: red. 
cluster to the $\mathrm{Si}_{16} \mathrm{Ta}$ nanoparticle. Thus, the dimer was not further investigated in a potential bulk structure.

To explain this occurrence, the first ionization potential and electron affinity of $\mathrm{Si}_{16} \mathrm{Ta}$ have been calculated and found to be $5.83 \mathrm{eV}$ and $-2.53 \mathrm{eV}$ respectively. Moreover, in chapter $[3$ it has been mentioned that all gold clusters reveal a low first IP due to high polarizability. Therefore the clusters that should, in terms of the superatomic concept, act as electron acceptors can also be used as electron donors when they are close to a cluster that reveals a large enough electron affinity. This seems to exactly be the case in this particular situation. The first IP for the spin polarized gold cluster has been found to be $4.93 \mathrm{eV}$, whereas the EA reveals a value of $-2.99 \mathrm{eV}$. Comparing the first IP and EA of both clusters shows a slightly more favored electron transfer from $\mathrm{Au}_{13}\left[\mathrm{RS}(\mathrm{AuSR})_{2}\right]_{6}$ to the Si cluster, since the sum of both values leads to $2.4 \mathrm{eV}$ that still need to be overcome in the case of a charge transfer to the $\mathrm{Si}_{16} \mathrm{Ta}$ particle, whereas the sum of the IP of the Si cluster and the EA of the undoped gold cluster results in $2.84 \mathrm{eV}$, thus more energy is needed.

To prove that a gold superatom can act as an electron acceptor, a $\mathrm{Au}_{13}\left[\mathrm{RS}(\mathrm{AuSR})_{2}\right]_{6}$ cluster has been relaxed in the presence of an individual sodium atom. The sodium atom was found to be preferably located between the gold cluster ligands close to the cluster core similar to the borane containing dimer. The binding energy between cluster and atom has been calculated to be $-2.10 \mathrm{eV}$ (-2.38 eV including dispersion). Furthermore the desired charge transfer has been observed with the sodium atom revealing a Bader charge of 0.86 in both cases (PBE and PBE $+\mathrm{D} 3)$.

A similar calculation with $\mathrm{Au}_{12} \mathrm{Pd}\left[\mathrm{RS}(\mathrm{AuSR})_{2}\right]_{6}$-Mg resulted in no charge transfer with a Bader charge of $0.02 \mathrm{e}$, caused by the high ionisation potential of $\mathrm{Mg} . \mathrm{Au}_{12} \mathrm{Pd}\left[\mathrm{RS}(\mathrm{AuSR})_{2}\right]_{6}$ which should in theory be a two electron acceptor cluster, exhibits such a low first and in particular second electron affinity that the probability of this cluster to receive two additional electrons is very low. Nonetheless, the dimer interaction between the sodium atom and the undoped gold cluster shows the possibility of this cluster to act as an electron acceptor obtaining a superatomic eight electron $\mathrm{P}$ shell closing. It has to be kept in mind that the first IP of the undoped gold cluster is so low that it can even act as electron donor. 


\subsubsection{Superatomic Gold Clusters as Electron Donor/Acceptor Building Blocks}

It has been shown that a single atomic impurity can have a large impact on the electronic structure of a small cluster. Moreover, the last passages introduced clusters that should according to the superatom model behave similarly to an alkali atom and a individual halogen atom. Verification of this concept has been obtained using different acceptor or donor clusters/atoms. To support this idea and to show that even the gold clusters themselves can act with one another as a donor acceptor pair, a dimer consisting of $\mathrm{Au}_{12} \mathrm{Al}\left[\mathrm{RS}(\mathrm{AuSR})_{2}\right]_{6}$ and $\mathrm{Au}_{13}\left[\mathrm{RS}(\mathrm{AuSR})_{2}\right]_{6}$ has been chosen for investigation. Table

Table 5.5: Cohesive energies $\mathrm{E}_{\mathrm{coh}}$ and Bader charges $\mathrm{q}$ of the undoped gold clusters calculated for the dimer $\mathrm{Au}_{13}\left[\mathrm{RS}(\mathrm{AuSR})_{2}\right]_{6}-\mathrm{Au}_{12} \mathrm{Al}\left[\mathrm{RS}(\mathrm{AuSR})_{2}\right]_{6}$, using the functional PBE and PBE + Grimme's D3 correction.

\begin{tabular}{ccccc}
\hline \hline & \multicolumn{2}{c}{ PBE } & \multicolumn{2}{c}{ PBE+D3 } \\
dimer & Bader charge $[\mathrm{q}]$ & $\mathrm{E}_{\mathrm{coh}[\mathrm{eV}]}$ & Bader charge $[\mathrm{q}]$ & $\mathrm{E}_{\text {coh }[\mathrm{eV}]}$ \\
\hline $\mathrm{Au}_{13}$ cluster- $\mathrm{Au}_{12} \mathrm{Al}$ cluster & 0.24 & -0.01 & 0.47 & -0.64 \\
\hline \hline
\end{tabular}

5.5 lists the cohesive energy values and Bader charges obtained for the relaxed dimer structure with and without dispersion correction. Similar to the case of the fullerenegold cluster dimer a low binding energy and low charge transfer due to a large distance between the clusters is obtained for the PBE converged dimer. The dispersion corrected dimer however, reveals relatively high binding energy and a high charge transfer indicating the importance of the dispersion interaction regarding ligand protected systems. In the case of the fullerene containing structure it has been shown that nonetheless a high binding energy and high charge transfer can be achieved through short distances in the lattice that are not preferred in the dimers. In this particular case even high binding energies are obtained for the dispersion corrected dimer. Thus, both gold clusters have been calculated in a binary cubic CsI type structure due to their nearly similar sizes. Figure 5.11 shows the bulk structure of $\mathrm{Au}_{12} \mathrm{Al}\left[\mathrm{RS}(\mathrm{AuSR})_{2}\right]_{6}-\mathrm{Au}_{13}\left[\mathrm{RS}(\mathrm{AuSR})_{2}\right]_{6}$ obtained after relaxation from an initial cubic CsI type structure while Table 5.3 summarizes the Bader charge of the $\mathrm{Al}$ doped gold cluster, the cohesive energy and the lattice parameters calculated. The values in Table 5.3 show that even though the dimer interaction 


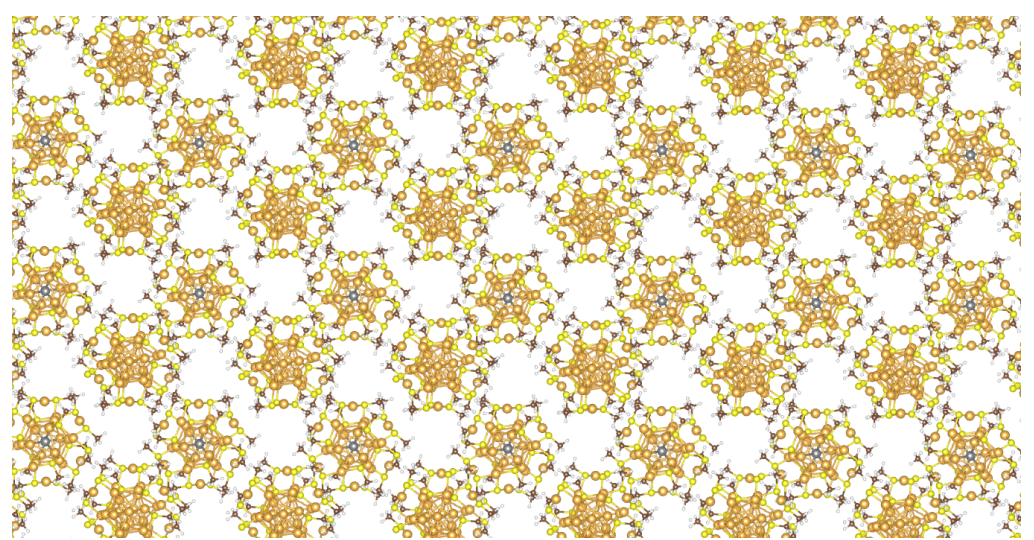

Figure 5.11: Visualization of the $\mathrm{Au}_{12} \mathrm{Al}\left[\mathrm{RS}(\mathrm{AuSR})_{2}\right]_{6}-\mathrm{Au}_{13}\left[\mathrm{RS}(\mathrm{AuSR})_{2}\right]_{6}$ relaxed cubic CsI like bulk structure.

Table 5.6: Cohesive energies $\mathrm{E}_{\mathrm{coh}}$ and Bader charges of the $\mathrm{Al}$ doped gold cluster $\mathrm{q}$ for $\mathrm{Au}_{12} \mathrm{Al}\left[\mathrm{RS}(\mathrm{AuSR})_{2}\right]_{6}-\mathrm{Au}_{13}\left[\mathrm{RS}(\mathrm{AuSR})_{2}\right]_{6}$ in a relaxed cubic CsI like bulk structure.

\begin{tabular}{|c|c|c|c|c|c|c|}
\hline & \multicolumn{3}{|c|}{$\mathrm{PBE}$} & \multicolumn{3}{|c|}{$\overline{\mathrm{PBE}+\mathrm{D} 3}$} \\
\hline Bader charge $[q]$ & \multicolumn{3}{|c|}{0.47} & \multicolumn{3}{|c|}{0.69} \\
\hline $\mathrm{E}_{\mathrm{coh}}[\mathrm{eV}]$ & \multicolumn{3}{|c|}{-1.9} & \multicolumn{3}{|c|}{-6.32} \\
\hline lattice parameters $[\AA]$ & $\mathrm{a}$ & $\mathrm{b}$ & $\mathrm{c}$ & $\mathrm{a}$ & $\mathrm{b}$ & c \\
\hline & 17.74 & 18.04 & 17.60 & 16.73 & 15.57 & 15.82 \\
\hline
\end{tabular}


has found to be weak in the case of the PBE calculation, a rather high cohesive energy is achieved for the assembled material. In addition, the expected charge transfer can be seen. The lattice parameters only deviate slightly from each other showing that a nearly cubic structure is maintained. The angles between the lattice vectors were found to be 90.9-91. $7^{\circ}$, thus very close to $90^{\circ}$ as in cubic primitive cells. Regarding the dispersion corrected assembly calculation, a large increase in binding energy has been found, which is reemphasizing the importance of the dispersion interactions in those systems. Furthermore the size of the unit cell, thus the distance between the interacting clusters was found to decrease strongly. To get more insight into the electronic situation of this assembly,

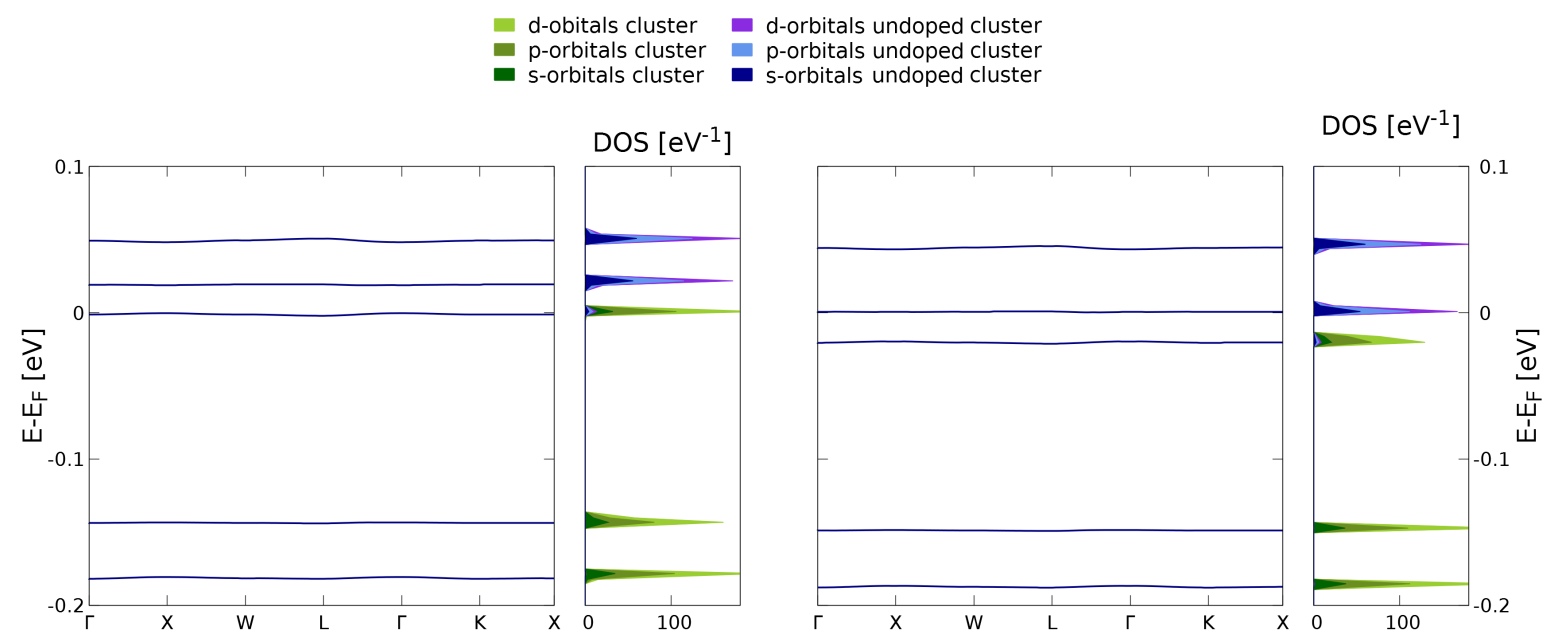

Figure 5.12: Visualization of the band structure and density of states of the CsI like gold cluster assembly for the majority and minority spin. Calculated along a cubic cell high symmetry path. The image on the right hand side reaveals an impurity state at $\sim 0 \mathrm{eV}$.

the band structure has been calculated and is visualized in Fig. 5.72. In comparison to the previously discussed assemblies, the the $\mathrm{Au}_{12} \mathrm{Al}\left(\mathrm{RS}(\mathrm{AuSR})_{2}\right)_{6}-\mathrm{Au}_{13}\left(\mathrm{RS}(\mathrm{AuSR})_{2}\right)_{6}$ bands are found to be the flattest, indicating very localized states. Moreover, the HOMO state is found to be centered at the anion of the bulk $\left(\mathrm{Au}_{13}\left[\mathrm{RS}(\mathrm{AuSR})_{2}\right]_{6}\right)$, as it is the case in atom based ionic solids. Despite the ionic character the HOMO-LUMO gap was found to be smaller than in the case of the previously presented bulk structures, namely $\sim 20-70 \mathrm{meV}$. 


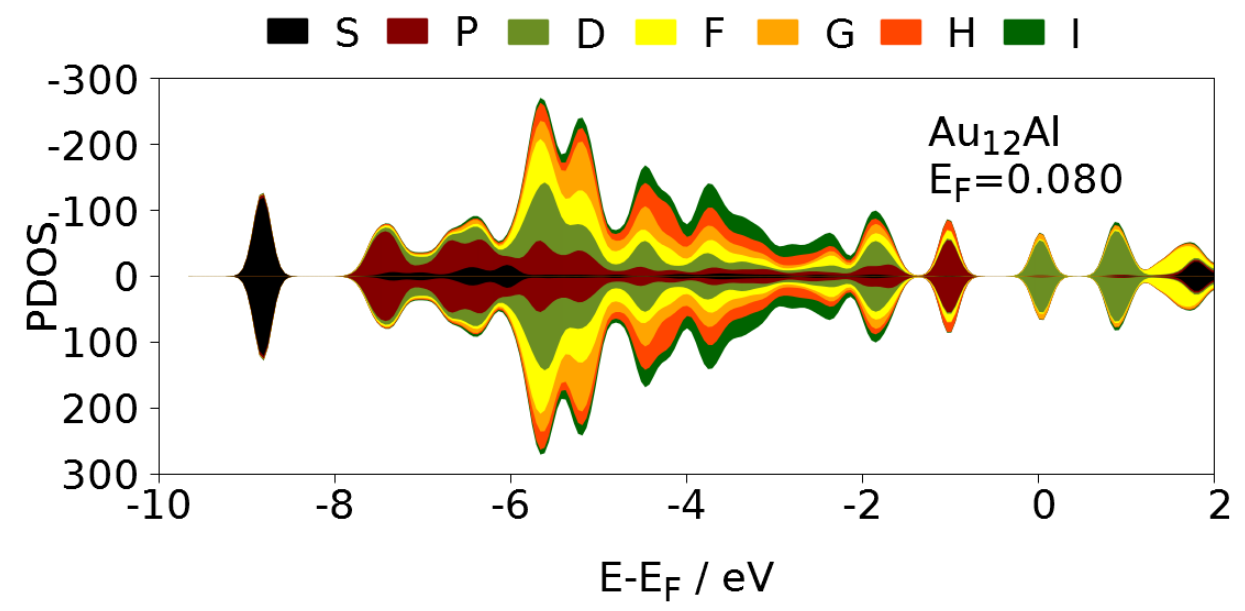

Figure 5.13: Visualization of the PDOS in a sphere around the cluster core of $\mathrm{Au}_{12} \mathrm{Al}\left[\mathrm{RS}(\mathrm{AuSR})_{2}\right]_{6}$ in structure $5 . \mathrm{Tl}$.

The PDOS around cluster $\mathrm{Au}_{12} \mathrm{Al}\left[\mathrm{RS}(\mathrm{AuSR})_{2}\right]_{6}$ depicted in Fig. 5.13 again shows the superatomic nature of the compound as discussed in the other two cases before.

In order to investigate if the ligands haven an influence on the interaction of the superatomic orbitals and the charge transfer, the differences occurring when removing the ligands has been studied. The first case that has been investigated is the relaxation of $\mathrm{Au}_{12} \mathrm{Al}$ and $\mathrm{Au}_{13}$ in a CsI type structure excluding ligands. Figure 5.14 depicts the obtained structure. It can be seen that the clusters coalesce in a bulk like structure while each cluster is keeping some of the original shape. The high binding energies of -2.03 $\mathrm{eV} /$ atom $(\mathrm{PBE})$ and $-2.96 \mathrm{eV} /$ atom $(\mathrm{PBE}+\mathrm{D} 3)$ speak for an actual coalescence. The fcc bulk gold value calculated using a similar functional is found to be $-3.25 \mathrm{eV} /$ atom (taken from Ref. ${ }^{[102]}$ ) and thus, higher then in the case of the cluster coalescence. This indicates that the clusters in the bulk material still retain some "cluster character". Despite the binding energy, the Bader charges regarding $\mathrm{Au}_{12} \mathrm{Al}$ and $\mathrm{Au}_{13}$ have both been calculated as separated clusters. As in the case of the ligand protected species a charge transfer from the $\mathrm{Al}$ containing cluster to the pure gold cluster can be observed. Nevertheless, both cases are not directly comparable in the context of their superatomic character, due to the fact that neglecting the ligands changes the amount of superatomic electrons present in each cluster, leading to partially filled D shells in the case of both clusters 


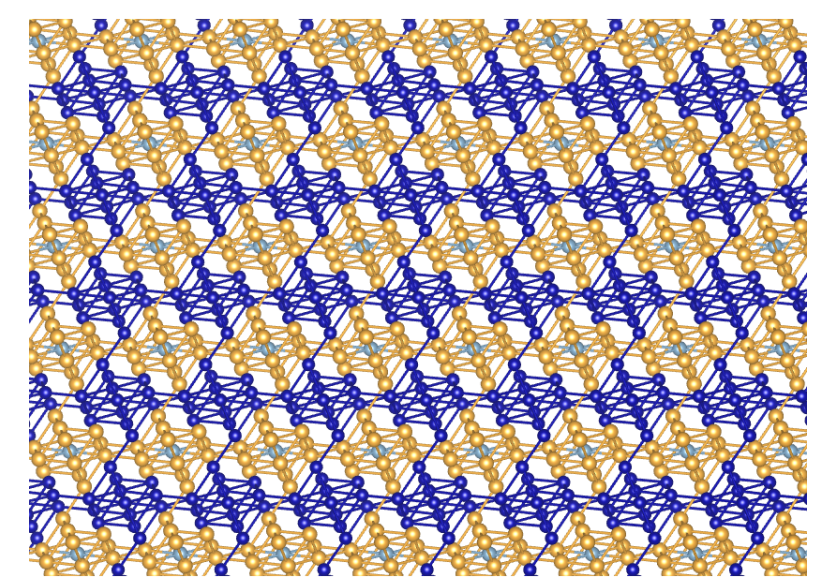

Figure 5.14: Visualization of the ligand unprotected $\mathrm{Au}_{12} \mathrm{Al}$ and $\mathrm{Au}_{13}$ cluster after relaxation from an initial cubic CsI like bulk structure. The gold atoms of cluster $\mathrm{Au}_{13}$ are shown in blue.

$\left(\mathrm{Au}_{12} \mathrm{Al}\right.$ and $\left.\mathrm{Au}_{13}\right)$.

The other case that was supposed to be investigated is based on even larger ligands. A bulk structure based on thiolate protected gold clusters revealing phenyl groups instead of methyl groups was found to be not computationally feasible. Larger ligands however, have been investigated regarding individual clusters in chapter 6 .

\subsubsection{Comparison}

In order to understand the different binding situations in the investigated dimers and solids, the relationship between different properties is analyzed. Fig. 5.15 contains the absolute value of the charge transfer found between the cluster dimers plotted as a function of the cohesive energy. The plot reveals that in the dimers, exhibiting clusters of relatively small size difference (gold cluster-fullerene and gold cluster-gold cluster), show an increasing cohesive energy with increasing charge transfer. In the case of $\mathrm{Au}_{13}\left[\mathrm{RS}(\mathrm{AuSR})_{2}\right]_{6}-\mathrm{C}_{60}$ an increase of the cohesive energy, however, only leads to a very slight increase of charge transfer due to the high IP value of the undoped gold cluster. Furthermore, it can be seen that the values for the gold cluster-fullerene dimers all lie in the low left end of the plot revealing low charge transfer and low binding energies, as the values for $\mathrm{Au}_{13}\left[\mathrm{RS}(\mathrm{AuSR})_{2}\right]_{6}-\mathrm{Au}_{12} \mathrm{Al}\left[\mathrm{RS}(\mathrm{AuSR})_{2}\right]_{6}$ do. In the case of the latter 


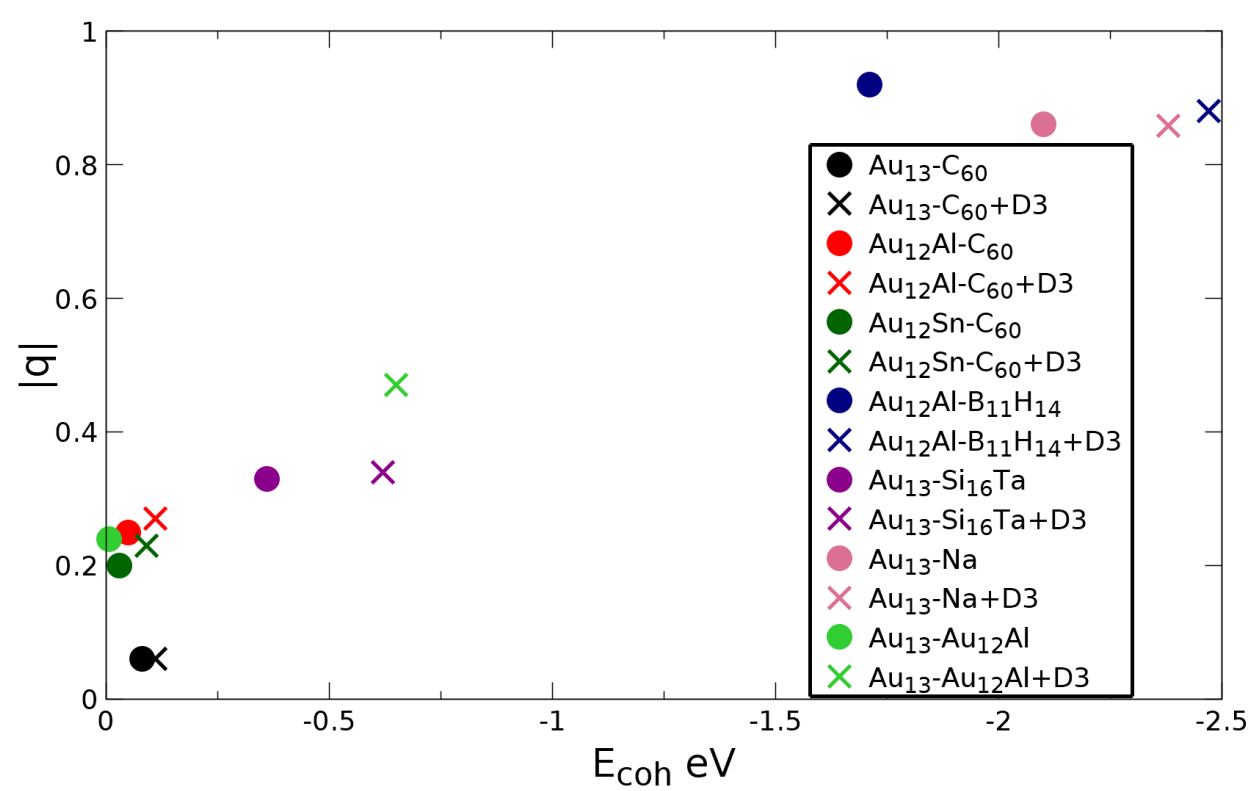

Figure 5.15: Visualization of the absolute value of the charge transfer $|\mathrm{q}|$ (Bader charge) plotted against the cohesive energy $\mathrm{E}_{\mathrm{coh}}$ calculated for the different dimers. All PBE obtained values are marked with circles while the calculations including D3 dispersion correction are visualized with crosses.

however a strong increase of the binding energy can be observed, when dispersion interactions are considered. The $\mathrm{Si}_{16} \mathrm{Ta}$ is smaller in size than the fullerene and depicts an intermediate case with a larger relative size difference of the clusters in the dimer (gold cluster- $\mathrm{Si}_{16} \mathrm{Ta}$ ). The values corresponding the dimers revealing large size difference (gold cluster-boran and gold cluster-Na) can be found in the upper far right corner of the graphic exhibiting high binding energies and high charge transfer. Another remarkable aspect is the slight decrease of the charge transfer with increasing binding energy that can be observed for the $\mathrm{Au}_{12} \mathrm{Al}\left[\mathrm{RS}(\mathrm{AuSR})_{2}\right]_{6}-\mathrm{B}_{11} \mathrm{H}_{14}$ and $\mathrm{Au}_{13}\left[\mathrm{RS}(\mathrm{AuSR})_{2}\right]_{6}$-Na dimer. In the case of the borane containing dimer, this effect has already been mentioned as a potential weakness of the Bader approach: A slight reduction of the overall charge transfer due to a competition with the occurring dispersion interactions.

Moreover, it can be shown that the mentioned size ratio of the investigated dimers is in good agreement with the idea that a distance decrease between the clusters results in a larger binding energy. Fig. 5.16 depicts such a correlation between the binding energy 


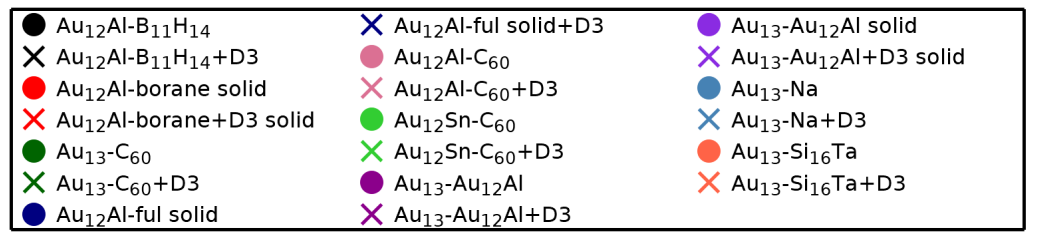

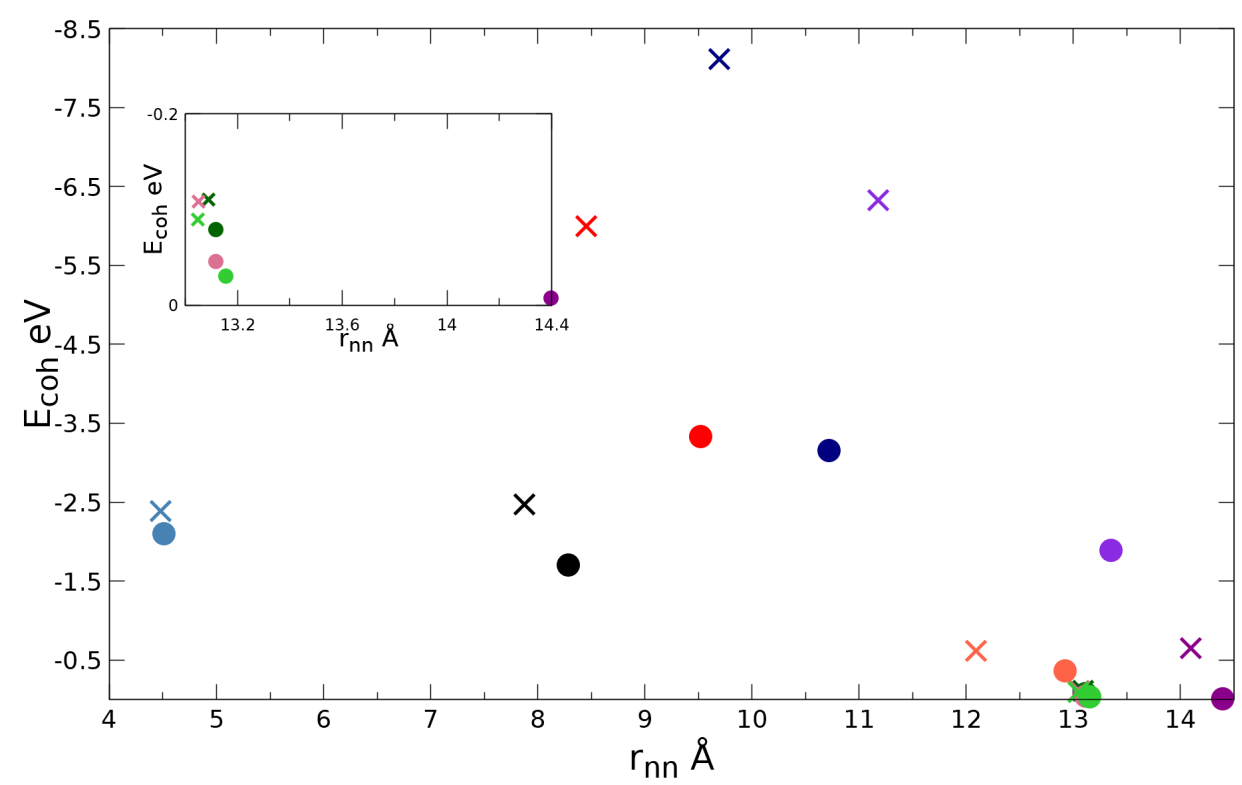

Figure 5.16: Visualization cohesive energy $\mathrm{E}_{\text {coh }}$ plotted as a function of the nearest neighbor distance $r_{n n}$ for the different calculated dimers and solid-state materials, while $r_{n n}$ has been calculated as the distance between the centers of the regarded clusters. All PBE obtained values are marked with circles while the calculations including D3 dispersion correction are visualized with crosses.

$\mathrm{E}_{\mathrm{coh}}$ and the nearest neighbor distance $\mathrm{r}_{\mathrm{nn}}$. The $\mathrm{x}$-axis was chosen to be the nearest neighbor distance in order to visualize the corresponding bulk structure values in the same plot.

Taking again a look at the $\mathrm{E}_{\mathrm{coh}}-\mathrm{r}_{\mathrm{nn}}$ correlation found for the dimers again shows the possibility to group the dimers regarding their size ratio. The gold cluster-fullerene and gold cluster-gold cluster containing dimers can all be found to have large distances and relatively low cohesive energies. The distance between the dimers dramatically shortens in the case of the borane and $\mathrm{Na}$ atom containing dimers leading to large binding energies. The images presented in Figures 5.1.B and 5.10. B have already shown that small clusters without large ligands are able to approach the cluster core, which leads to an increased 
charge transfer. In addition, a reduction of the nearest neighbor distance in the case of all solids except for the borane containing bulk is found, reemphasizing that the binding situation changes when considering solids. In the case of the $\mathrm{Au}_{12} \mathrm{Al}\left[\mathrm{RS}(\mathrm{AuSR})_{2}\right]_{6^{-}}$ $\mathrm{B}_{11} \mathrm{H}_{14}$ solid a distance increase relative to the dimer can be observed simply due to the fact that each $\mathrm{Al}$ doped gold cluster is tetrahedrally coordinated by four $\mathrm{B}_{11} \mathrm{H}_{14}$ making it impossible for the ligands to make space for every borane cluster to approach the cluster core. In consequence, evenly distributed ligands are favored. Nevertheless a higher cohesive energy can be found in the case of the $\mathrm{Au}_{12} \mathrm{Al}\left[\mathrm{RS}(\mathrm{AuSR})_{2}\right]_{6}-\mathrm{B}_{11} \mathrm{H}_{14}$ solid showing the importance of the lattice energy. An other aspect that can be found nicely visualized in Fig. 5.16 is the decrease of the distance between cluster with the inclusion of dispersion corrections and thus an increase of the binding energy. Furthermore it can

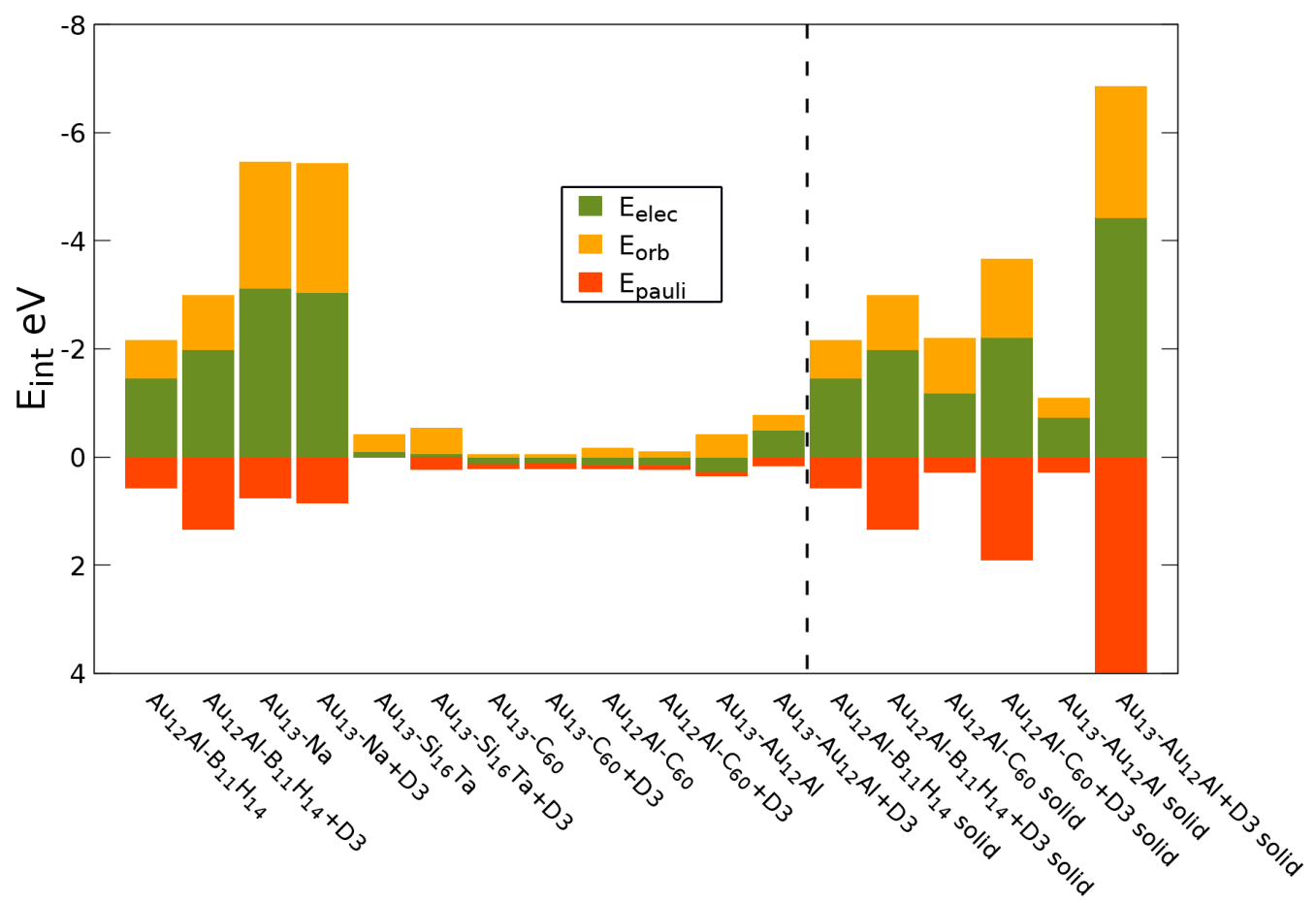

Figure 5.17: Visualization the different contributions to the interaction energies $\mathrm{E}_{\text {int }}$ present in the clusters dimers: electrostatic interaction $\mathrm{E}_{\text {elec }}$, orbital interaction $\mathrm{E}_{\text {orb }}$

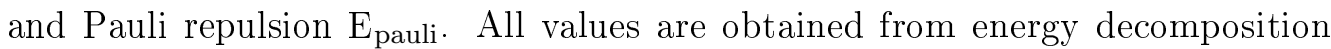
analysis. As representative for the dimer interaction present in the solids the nearest neighbor pair has been chosen and presented on the right hand side of the graphic. 
be seen that $\mathrm{E}_{\text {coh }}$ strongly increases with the inclusion of the D3 correction in the case of the $\mathrm{Au}_{12} \mathrm{Al}\left[\mathrm{RS}(\mathrm{AuSR})_{2}\right] \mathrm{C}_{60}$ and $\mathrm{Au}_{12} \mathrm{Al}\left[\mathrm{RS}(\mathrm{AuSR})_{2}\right]_{6}-\mathrm{Au}_{13}\left[\mathrm{RS}(\mathrm{AuSR})_{2}\right]_{6}$ solid. In the case of the latter a strong increase in binding energy can already be found for the dimer. This again emphasizes the importance of dispersion interactions in ligand protected and molecular systems.

A detailed analysis of the interaction energy between the different dimers can be found in Fig. 5.17, splitting $E_{\text {int }}$ up into the electrostatic interaction $E_{\text {elec }}$, the orbital contribution $\mathrm{E}_{\text {orb }}$ and the Pauli repulsion $\mathrm{E}_{\text {pauli }}$ (For more detailed description please see $[2.3)$. The dispersion interaction is not considered in this Figure. The results are in good agreement with Fig. 5.16 and 5.15. The interaction energy for all fullerene containing, the $\mathrm{Si}_{16} \mathrm{Ta}$ containing and the gold-gold dimers is found to be low or even slightly repulsive. The orbital overlap is the sole attractive interaction between the fullerene containing dimers, whereas the $\mathrm{Si}_{16} \mathrm{Ta}$ containing dimers and the dispersion corrected gold-gold dimer reveal a slightly attractive electrostatic interaction. In the case of the $\mathrm{Au}_{12} \mathrm{Al}\left[\mathrm{RS}(\mathrm{AuSR})_{2}\right]_{6}-\mathrm{B}_{11} \mathrm{H}_{14}$ and $\mathrm{Au}_{12} \mathrm{Al}\left[\mathrm{RS}(\mathrm{AuSR})_{2}\right]_{6}$-Na dimers a large interaction energy is found which is dominated by the electrostatic contribution to binding. Thus, in the case of the clusters with smaller size ratio the interaction is found to be mostly caused by orbital interaction while the interaction in the case of the large size ratio dimers (one small cluster/atom without large ligands) is found to be dominated by ionic interactions. Considering the solids, the electrostatic interaction dominates in each case while there is still a large orbital contribution present. The latter is highly important when thinking of electronically active bulk materials. The ratio orbital contribution to electrostatic interaction is much higher in the case of the fullerene containing solid compared to both other solid state materials. This might indicate that the orbital interaction or orbital interaction/electrostatic interaction ratio can directly be related to the degree of band dispersion.

\subsection{Conclusion}

The high tunability of the electronic structure of small thiolate protected gold clusters (here in particular $\mathrm{Au}_{13}\left[\mathrm{RS}(\mathrm{AuSR})_{2}\right]_{6}$ ), due to the ability to use single atomic impurities 
to alter the electronic structure such that those clusters resemble individual atoms (e.g. halogens or alkali metals), led to the idea to investigate those clusters as building block for bulk materials.

The analysis presented in this chapter ranges from dimer interactions between clusters to carefully chosen binary cluster based bulk structures with one of the components being a doped or undoped $\mathrm{Au}_{13}\left[\mathrm{RS}(\mathrm{AuSR})_{2}\right]_{6}$ cluster in each case. The gold cluster was investigated with clusters of different chemical composition, electronic structure and size. It has been shown that the gold cluster can indeed act similarly to a halogen atom as electron acceptor or as an alkali metal, thus as an electron donor. Due to the low ionization potential of the clusters and the easily tunable electron affinity, the binding partner has to be considered carefully to obtain desired properties, such as charge transfer, in a specific direction.

Furthermore it has been shown that in the case of dimers containing small ligand unprotected clusters or atoms, the latter both are preferably located between the ligands approaching the cluster core. This leads to a strong binding interaction with a large electrostatic contribution and therefore also a large charge transfer. Combining the doped $\mathrm{Au}_{13}\left[\mathrm{RS}(\mathrm{AuSR})_{2}\right]_{6}$ clusters with each other (large clusters with large ligands) or fairly large molecules such as buckyballs with the gold cluster results in rather low dimer binding energies and low charge transfer, while the attractive interaction energy seems to be dominated by orbital overlap in all cases.

In summary, the relative size of the clusters and the ligands play a central role in defining the inter-cluster interaction present in dimers. Two very different possible binding situations have been identified.

Subsequently three different solid-state materials have been chosen for calculation. These bulks include both types of of dimer interactions that were previously found, and the gold cluster acting as both electron donor and electron acceptor. The superatomic character of these materials has been confirmed by showing that the transferred charge is contributing to or taken from the superatomic charge of the gold cluster in each system. Moreover it has been shown, that even in the case of low dimer interactions high solidstate binding energies can be achieved. The clusters confined in a fixed lattice usually reveal a shorter nearest neighbor distance when the binary assembly consists of clusters 
with similar size. This leads to a larger orbital overlap and when the clusters are chosen carefully regarding their IP and EA values, in a high charge transfer. In each of the investigated structures the electrostatic interaction was found to be dominating while a large attractive orbital interaction is achieved. Both contributions, in particular the latter are important when thinking of electronically active materials.

Another interesting finding shows that the dispersion of the bands of solid state materials can be related to the degree of orbital interaction present. 


\section{Ligands Protecting the Clusters}

\subsection{Introduction}

The previous chapters discussed various factors that affect potential superatomic assemblies such as the tunability of the electronic structure, the IP and EA values and relative sizes of the clusters. Furthermore each of the chapters mentioned the ligands protecting the clusters as one of the main factors that need to be considered. Chapter 3 pointed out that the ligands play an important role in creating the superatomic states, while chapter 2 showed that they are important when looking at potential charge transfer between the clusters. The last chapter furthermore introduced the idea of structural and dispersive interactions of the clusters based on the ligands. In order to create a complete picture, the following chapter intends to investigate different kinds of ligands that have experimentally been used. Furthermore some additional theoretical ligands have been added to the investigation to analyze the effect of the size and type of the ligands.

Previous studies have reported that thiolate protected gold clusters reveal higher stability then phosphine protected clusters ${ }^{[0,103]}$. Even more recent investigations have shown that selenolate protected clusters exhibit even higher stability and are more stable against degradation in solution ${ }^{[194]}$. Beyond that a few theoretical studies have been conducted to investigate the stability of clusters protected with e.g. different thiolate ligands, thus the effect of the ligands ${ }^{[19.2]}$.

The work presented in this chapter also contains a stability analysis, while in contrast to other studies for example the different experimentally known ligands (thiolate, phosphine and selenolate) are investigated and compared. Furthermore the size and type of the ligands is analyzed regarding their use in potential superatomic solid-state materials. 


\subsection{Methods}

All calculations discussed in this chapter have been conducted with the program VASP. The input structures for the experimentally known clusters have been obtained from Ref.s ${ }^{[}$, II] $]$while the other cluster structures have been manually constructed. In all cases a full structure relaxation without symmetry restrictions has been performed using the functional PBE and PBE + D3 with Becke-Johnson damping ${ }^{[134]}$. The energy convergence criterion was set to $10^{-5} \mathrm{eV}$ and the ionic relaxation was stopped when all forces dropped below $0.01 \mathrm{eV} / \AA$. Furthermore the Brillouin zone integration was done employing Gaussian smearing with a smearing factor of 0.1. For the charged clusters a full relaxation has been performed in all cases. The energies of the cluster fragments have been obtained by conducting single point calculations on the fragment structure as obtained from the full cluster relaxation.

\subsection{Discussion}

Fig. 6.7 shows a visualization of the $\mathrm{Au}_{13}$ clusters with different ligand shells that have been investigated regarding their stability and application as building block for assemblies. The clusters chosen to be studied are the experimentally known phosphine protected cluster A exhibiting two additional chlorine ligands, the thiolate protected cluster B and the selenolate protected cluster C. In order to additionally compare the influence of the size of the ligand all organic groups attached to the $\mathrm{P}, \mathrm{S}$ or Se atoms have been replaced with methyl groups. The resulting model clusters can be found in Fig. 6.5 under a, b and c. To additionally include the effect of stronger electron withdrawing ligands, a $\mathrm{Au}_{13}$ cluster protected by iso-propoxide moieties (see 6.1.d) has been constructed and investigated. The terms cluster A, B, C and a, c, b, d will be continued to be used throughout this chapter when it is referred to the cluster with equivalent ligands as visualized in Fig. 6.]. In order to study those clusters from an energetic point of view, thus the stability of the clusters, the total interaction energy of each cluster can be defined as: $E_{\text {total }}=E_{\text {core }}+E_{\text {core-lig }}+E_{\text {lig-lig }}+E_{\text {lig }}$. In this equation $E_{\text {core represents }}$ the binding energy of the cluster core which is obtained by subtracting the energy of 


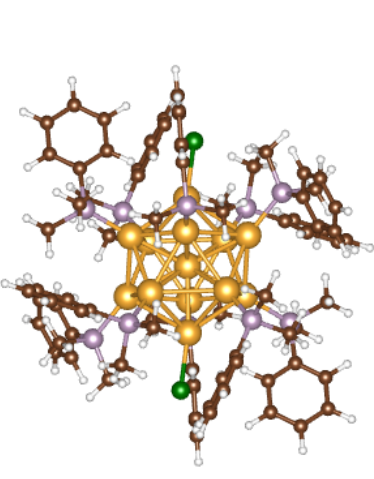

A

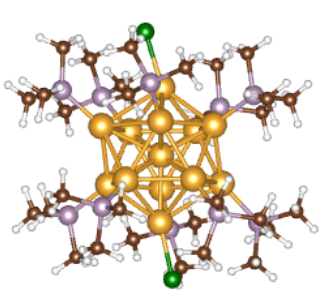

a

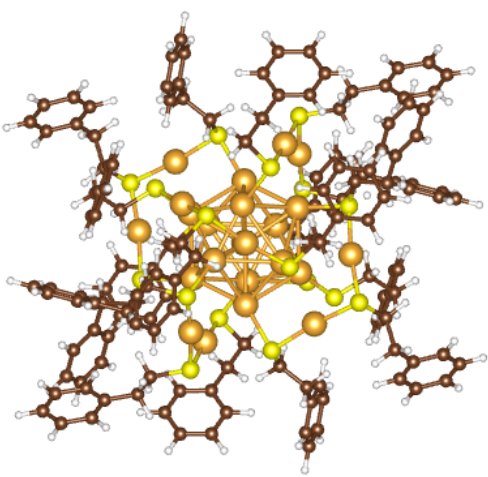

$\mathrm{B}$

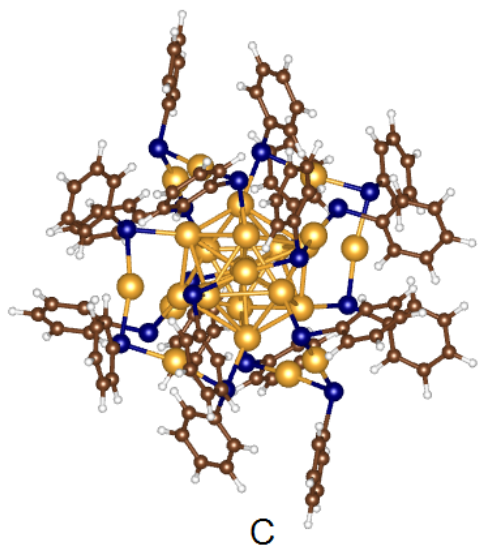

C

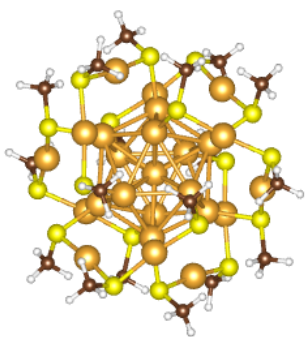

b

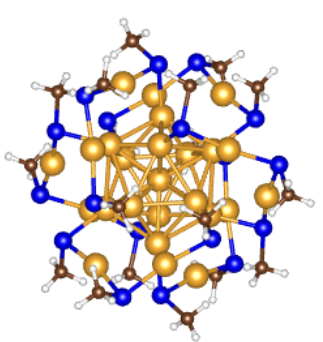

c

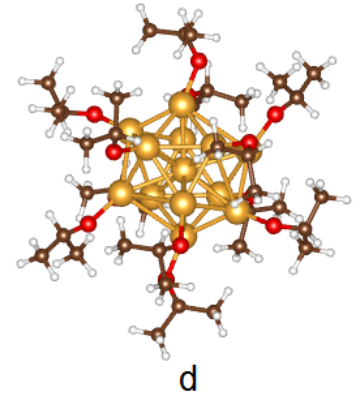

A: $\quad \mathrm{Au}_{13} \mathrm{Cl}_{2}\left(\mathrm{PPh}\left(\mathrm{CH}_{3}\right)_{2}\right)_{10}$, B: $\mathrm{Au}_{13}\left[\mathrm{PhCH}_{2} \mathrm{CH}_{2} \mathrm{~S}\left(\mathrm{AuSPhCH}_{2} \mathrm{CH}_{2}\right)_{2}\right]_{6}, \quad \mathrm{C}: \mathrm{Au}_{13}\left[\mathrm{PhSe}(\mathrm{AuSePh})_{2}\right]_{6}, \quad$ a: $\mathrm{Au}_{13} \mathrm{Cl}_{2}\left(\mathrm{P}\left(\mathrm{CH}_{3}\right)_{2}\right)_{10}$, b: $\mathrm{Au}_{13}\left[\mathrm{CH}_{3} \mathrm{~S}\left(\mathrm{AuSCH}_{3}\right)_{2}\right]_{6}$, c: $\mathrm{Au}_{13}\left[\mathrm{CH}_{3} \mathrm{Se}(\mathrm{AuSeCH})_{2}\right]_{6}$, d: $\mathrm{Au}_{13}\left({ }^{i} \mathrm{PrO}\right)_{12}$. Color code: Au: orange, P: gray, S: yellow, Se: blue, Cl: green, $\mathrm{O}$ : red, $\mathrm{C}$ : brown and $\mathrm{H}$ : white. 
each individual gold atom from the core calculated in the structure obtained from the whole cluster relaxation. $E_{\text {core-lig }}$ is nothing else but the interaction energy between the cluster core and the ligands calculated by subtracting the energy of the ligand shell and the energy of the core from the total energy of the whole cluster. $E_{\text {lig-lig }}$ is defined to be the interaction energy between the ligands obtained by subtracting the energy of each ligand from the energy of the ligand shell. All fragment calculations are single point calculations conducted on the structure of the fragments obtained from the whole cluster relaxation. The fourth contribution to the total interaction energy is the internal binding energy of each ligand $E_{\text {lig. }}$. The latter can be neglected since it is caused by strong covalent bonds and therefore does not change the overall picture.

The individual contributions to the total interaction energy for each cluster are listed in Table 6.]. Each value has been obtained firstly with PBE, and then secondly, including dispersion interactions $(\mathrm{PBE}+\mathrm{D} 3)$. The values in Table $\mathbf{6 . 0}$ clearly show that the main

Table 6.1: Internal binding energy of the cluster core $E_{\text {core, }}$ interaction energy between the cluster core and the ligands $E_{\text {core-lig }}$ and interaction energy between the ligands $E_{\text {lig-lig }}$ for the seven different clusters in $\mathrm{eV}$.

\begin{tabular}{cccccccc}
\hline \hline & cluster A & cluster B & cluster C & cluster a & cluster b & cluster c & cluster d \\
\hline PBE & & & & & & & \\
$E_{\text {core }}$ & -24.50 & -24.76 & -24.69 & -24.37 & -24.76 & -24.73 & -25.06 \\
$E_{\text {core-lig }}$ & -19.36 & -20.88 & -19.75 & -20.03 & -20.99 & -20.60 & -21.60 \\
$E_{\text {lig-lig }}$ & -3.41 & -0.84 & -0.41 & -1.64 & -0.31 & -0.50 & 0.19 \\
\hline PBE + D3 & & & & & & & \\
$E_{\text {core }}$ & -26.82 & -27.11 & -27.04 & -26.70 & -26.97 & -27.08 & -27.25 \\
$E_{\text {core-lig }}$ & -23.65 & -26.26 & -25.27 & -23.79 & -25.94 & -25.52 & -24.08 \\
$E_{\text {lig-lig }}$ & -4.70 & -5.91 & -5.41 & -2.39 & -2.75 & -3.33 & -0.63 \\
\hline \hline
\end{tabular}

stability of the cluster comes from the cluster core and the binding of the ligands rather then the ligand-ligand interaction. This is in good agreement with the idea of the superatomic structure, which is emerging from the core and the core-ligand interaction, stabilizing the cluster. Test calculations conducted using different ligands such as hydroxyl groups and fully chlorinated clusters have resulted in a destruction of the cluster core structure. Thus, those results again emphasize the importance of choosing ligands of large enough size to protect the cluster and retain the icosahedral structure while not resulting in large steric or electrostatic repulsion between the ligands. 
The energy of the cluster cores varies slightly between the different clusters, since all of the cores reveal an almost perfect icosahedral structure. Interestingly the structure of the free, unprotected cluster core has been found not to exhibit an icosahedral structure, while showing the highest binding energies of $-25.92 \mathrm{eV}(\mathrm{PBE})$ and $-28.13 \mathrm{eV}(\mathrm{PBE}+\mathrm{D} 3)$. Nevertheless ligand unprotected clusters tend to coalesce (see chapter 5 ) since the studied ligands add more stability to the overall cluster. Thus, ligand unprotected clusters can not be considered as bulk building blocks.

Regarding the ligand core interaction it can be seen that the values are most strongly influenced by dispersion interaction. Furthermore it can be seen that the interaction is stronger for larger ligands. This is also the case for the ligand-ligand interaction which is mostly contributed to by dispersion interactions. However, the latter contribution is small compared to the other two, and does not add a lot of stability to the cluster. This is an important feature though when considering cluster assemblies since the results in chapter 5 showed that the binding energy of the solid state materials is largely dominated by interactions between the clusters. Therefore the ligands of each building block have to be able to to interact with the ligand shell of the other clusters, which can only be accomplished when the ligand-ligand interaction of each cluster is not too strong but strong enough to give additional stability. This is the case for all investigated clusters. In summary, long range interactions lead to a preference for larger ligands, thus for clusters A, B and C. B reveals the largest stability, however, it is obvious that the differences between the different clusters are quite small.

In addition it needs to be considered that the thiolate and selenolate ligands are binding at two cluster core surface atoms. The chelating behavior results in additional stability with ligands being reattached faster in the case of the dissociation from one binding site, thus an increased protection of the cluster core. Beyond that selenolate ligands compared to thiolate ligands have experimentally been found to increase the stability of the cluster against degradation in solution ${ }^{[194]}$. This speaks for large selenolate ligands, with strong contributions to dispersion interactions, as best candidates for the protection bulk building blocks, closely followed by large thiolate ligands.

Another measure of stability is given by the HOMO-LUMO gap of the clusters. Table 6.2 lists the HOMO-LUMO gaps for all seven clusters in their eight superatomic electron 
revealing species since the shell closed clusters should in theory be the clusters with the largest stability. The values of the phosphine and iso-propoxide protected clusters are

Table 6.2: HOMO-LUMO gap for the seven different clusters with charges corresponding to an superatomic $\mathrm{P}$ shell closing $(-1,+3$ and -7$)$.

\begin{tabular}{ccc}
\hline \hline cluster & cluster charge & HOMO/LUMO gap [eV] \\
\hline cluster A & +3 & 1.94 \\
cluster B & -1 & 1.21 \\
cluster C & -1 & 1.08 \\
cluster a & +3 & 2.03 \\
cluster b & -1 & 1.20 \\
cluster c & -1 & 1.25 \\
cluster d & -7 & $0.08-0.36$ \\
cluster without lig. & +5 & 1.15 \\
\hline \hline
\end{tabular}

not directly comparable with the values of the thiolate or selenolate protected nanoparticles, since the charge of the cluster is connected to the size of the gap. Comparing the clusters with the larger ligands to the smaller ligands reveals a decrease of the gap in the case of the $\mathrm{A}$ and $\mathrm{C}$ while the HOMO-LUMO gap between $\mathrm{B}$ and $\mathrm{b}$ remains quite similar. The clusters with the smaller ligands have the larger HOMO-LUMO gaps and thus enhanced chemical stability. The gap for cluster $d$ has been found to be 0.08 or 0.36 depending on the spin channels, which is relatively small, while the gap was found to be large in all other cases.

The importance of the electron affinity and ionization potential when thinking about electronically active building blocks and stable assemblies has been shown in chapter 5 . Therefore those electronic characteristics have been explored for the clusters revealing different ligands. Table 6.3 shows the 1st EA and IP values calculated as the total energy of the uncharged cluster subtracted from the singly negative or positive charged cluster respectively. The values listed reveal that the EA and IP can be influenced by the choice

Table 6.3: EA and IP values for the seven different clusters visualized in Fig. 6.D.

\begin{tabular}{cccccccc}
\hline \hline & cluster A & cluster B & cluster C & cluster a & cluster b & cluster c & cluster d \\
\hline 1st EA [eV] & -1.51 & -3.43 & -4.24 & -1.16 & -2.99 & -3.33 & -4.50 \\
1st IP [ev] & 2.41 & 4.12 & 3.46 & 1.16 & 4.12 & 4.02 & 5.59 \\
\hline \hline
\end{tabular}

of the ligand. Strongly electron withdrawing ligands seem to result in a larger IP value 
while the EA decreases. Thus, the potential application of clusters as electron donors or acceptors in a bulk material, can be fine tuned by choosing an electron withdrawing or donating ligand.

In order to discuss the involvement of the ligands in creating the superatomic orbitals a Bader charge analysis has been conducted and furthermore the radial distribution of the electron density of chosen orbitals has been investigated. The Bader charge values for the ligands of the seven clusters exhibiting a charge of $+1,0$ and -1 one can be found in Table 6.4. The charges reemphasize that additional charges, which contribute to the

Table 6.4: Bader charges in e for the cluster cores of the the seven different clusters visualized in Fig. 6.].

\begin{tabular}{cccccccc}
\hline \hline ligand charge & cluster A & cluster B & cluster C & cluster a & cluster b & cluster c & cluster d \\
\hline+1 & -1.55 & 0.63 & 0.04 & -1.70 & 0.60 & -0.03 & 3.74 \\
0 & -1.82 & 0.44 & -0.08 & -1.94 & 0.41 & -0.20 & 3.61 \\
-1 & -1.97 & 0.28 & -0.20 & -2.18 & 0.23 & -0.33 & 3.43 \\
\hline \hline
\end{tabular}

superatomic structure of the cluster, are mainly located at the ligands. This finding has been discussed in chapter 5 and has in this chapter been shown to apply for every ligand investigated. Furthermore, those charges reflect the electron withdrawing or donating character of the ligands.

Due to the electron donating character of the phosphine ligands, a larger charge separation between ligands and core is observable. Thus, the effect of the type of ligand on the superatomic orbitals of the clusters and potential orbital overlap in assemblies needs to be studied. Fig. [2.2 visualizes the radial charge distribution of the HOMO orbital for cluster $a, b$ and c. The clusters chosen for this Figure, have all been assigned the necessary charge in order to reveal P-shell closing. The graphic reveals the strong charge separation between the cluster core and the ligands, which has been already shown in the Bader charges, while the difference between cluster a and b is relatively small. However, in all cases the HOMO orbital has been found to be extended over the ligands. The positions marked on the x-axis of Fig. 6.2 indicate the distance from the gold cluster core center to the first atom of the next interacting cluster in the three investigated cluster assemblies. It can be seen that an orbital overlap with the nearest cluster would be established in the case of all three ligands. Therefore $\mathrm{a}, \mathrm{b}$ and $\mathrm{c}$ are all good candidates 


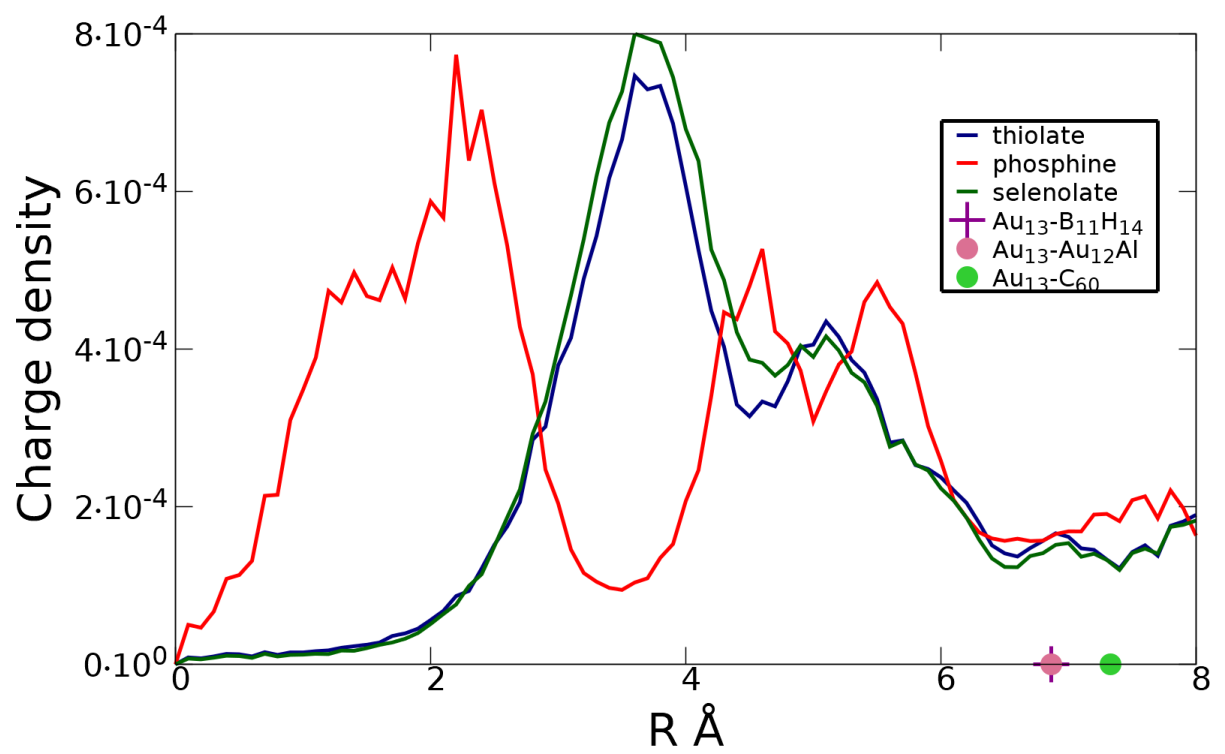

Figure 6.2: Radial charge distribution of the HOMO orbital of clusters $\mathrm{a}^{+3}, \mathrm{~b}^{-1}$ and $\mathrm{c}^{-1}$. The central atom of each cluster placed at $0 \AA$. The individual points indicate the distance to the closest atom of the closest interacting cluster in the binary assemblies investigated in chapter 1 .

as building blocks for functional materials, when only considering superatomic orbital overlap.

\subsection{Conclusion}

In this chapter different kinds of ligands protecting the same cluster core have been investigated. In summary it was found that the ligands play a large role in stabilizing the cluster core while the main stability is arising from the cluster core itself. The previous chapters have show the ligands protecting the gold cluster are essential to prevent clusters from coalescence, that they are important in creating the superatomic structure in particular the superatomic orbitals and can act as dielectric medium between clusters in cluster assemblies. This chapter has reemphasized most of the earlier findings and shown, that those also apply to other ligands.

In general it has been found that ligands play a central role while the exact choice of the ligand is less important considering superatomic assemblies. However, when choosing 
the ligands it has to be considered to choose large enough ligands to maintain the cluster core structure but also create a nanoparticle of desired size. Furthermore, it is important that a strong binding between the ligands and the cluster core is occurring while the ligand-ligand interaction has to be small enough to allow ligand flexibility. For chemical stability a large enough HOMO-LUMO gap is of interest. The ligand can also be chosen such that they tune the IP or EA in the desired direction. 


\section{Experimental Collaborations}

Besides the main project, two major experimental collaborations in cooperation with other research groups have been conducted and will be discussed in the following subsections.

\subsection{First Steps Towards the Understanding of $\mathrm{Au}_{9}\left(\mathrm{PPh}_{3}\right)_{8}-\mathrm{C}_{60}$ Compounds}

\subsubsection{Introduction}

The search for novel materials that exhibit interesting electronic properties, such as e.g. graphene ${ }^{[106-[198]}$, is central for the development of new technologies, thus always a hot topic. One approach to obtain new materials, that have relatively easy tunable properties, is the synthesis of superatomic cluster based materials. Chapter 1 shows that these kind of materials need to exhibit high charge mobility and sufficient orbital overlap to be considered of electronic interest. Furthermore it is demonstrated that these requirements can be achieved when choosing the interacting clusters carefully based on size, ligands and EA and IP values.

Driven by the same idea Fiedler et al. succeeded in the crystallization of a binary cluster compound consisting of $\mathrm{Au}_{9}\left(\mathrm{PPh}_{3}\right)_{8}$ and $\mathrm{C}_{60}$. The crystal was prepared by mixing a solution of $\mathrm{Au}_{9}\left(\mathrm{PPh}_{3}\right)_{8}$ in acetonitrile in a 2:1 ratio with $\mathrm{KC}_{60}$ dissolved in THF. Subsequent to the preparation an analysis using different techniques such as X-ray diffraction, TEM, UV-VIS and Raman spectroscopy followed.

Experimental characterization is a challenging procedure. Therefore theoretical work has been conducted in order to support the analysis. To get a first idea about the bind- 
ing situations and a potential charge transfer between clusters, a model system based on a rock-salt like crystal structure has been constructed. The underlying structure was chosen based on the relative sizes of the $\mathrm{Au}_{9}\left(\mathrm{PPh}_{3}\right)_{8}$ and $\mathrm{C}_{60}$ cluster (see chapter 5 ).

\subsubsection{Methods}

All calculations have been conducted using the program package Vienna Ab initio Simulation Package (VASP) 5.3.5. The input structures have been set up manually with a sensible distance between the clusters. A full structure relaxation has been performed in all cases with an energy convergence criterion of $10^{-4} \mathrm{eV}$. The ionic relaxation was stopped when all forces dropped below $0.02 \mathrm{eV} / \AA$ and the energy cut-off was chosen to be $500 \mathrm{eV}$. Due to the size of the unit cell and the fact that these calculations are only meant to give guidance towards the general binding situation e.g. a possible charge transfer, the Monkhorst-Pack $k$-mesh was chosen to a $2 \times 2 \times 2 \Gamma$-centered mesh. Furthermore the generalized gradient approximation PBE ${ }^{[129,[130]}$ was chosen as DFT functional and a Gaussian smearing with a smearing factor of 0.01 was utilized for the Brillouin zone integration. In addition the density of states has been calculated using a $6 \times 6 \times 6$ $k$-gird a smearing factor of 0.005 , while the respective band structure was calculated along an FCC high symmetry path. Moreover the XRD structure has been calculated based on the obtained VASP calculations using the software Diamond 4. In addition a EDA analysis using the software package ADF, the GGA functional PBE and a TZ basis set including 2 polarization functions has performed. Relativistic effects have been included using ZORA ${ }^{[179-181]}$.

\subsubsection{Discussion}

The crystalline material synthesized by Fiedler et al. was found to be amorphous and oxygen labile. Furthermore, the material was found to be very defective e.g. revealing polymerized buckyballs and excess gold clusters. In order to determine the most stable structure of this cluster assembly several measurements have been undertaken. The UVVIS spectrum of the sample was found to reveal new absorption maxima. In particular, two new maxima close to the main band of $\mathrm{Au}_{9}\left(\mathrm{PPh}_{3}\right)_{8}\left(\mathrm{NO}_{3}\right)_{3}$ were noticeable. UV-VIS 
calculations undertaken by Lukas Hammerschmidt helped to affiliate those bands to a spin splitting occurring in the $\mathrm{Au}_{9}\left(\mathrm{PPh}_{3}\right)_{8}^{+2}$ cluster. Thus, it has been concluded that the cluster assembly is mostly made up by the twice positively charged $\mathrm{Au}_{9}\left(\mathrm{PPh}_{3}\right)_{8}$ cluster.

Subsequently Raman spectra where measured. Since those vibrations are very sensitive to the charge of the observed molecule, a shift of the characteristics band associated with the $\mathrm{C}_{60}$ has been observed with proceeding reaction. The vibration measured for the reactant $\mathrm{KC}_{60}$ corresponds to singly negative charged buckyballs, while this band is shifted to higher frequency regarding the product. The shift of the band can be affiliated with a more positive charge of the $\mathrm{C}_{60} \mathrm{~S}$ incorporated in the produced material.

To support the idea of the crystalline material consisting of $\mathrm{Au}_{9}\left(\mathrm{PPh}_{3}\right)_{8}^{+2}$ and $\mathrm{C}_{60}^{-}$a model material consisting of a binary gold cluster fullerene assembly in a rock-salt structure has been constructed and fully relaxed. The rock-salt structure was chosen due to its simplicity and the relative sizes of the clusters. Due to the fact that the gold cluster is known to be most stable three times positively charged $\left(\mathrm{Au}_{9}\left(\mathrm{PPh}_{3}\right)_{8}^{+3}\right)^{[1 \times 6]}$ possible charge transfers between the bulk building blocks have been observed on the basis of Bader charges. Furthermore up to two $\mathrm{NO}_{3}$ molecules have been incorporated in the

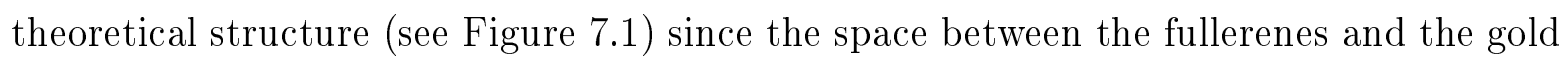
clusters leaves sufficient room for solvent molecules to occur. Moreover, $\mathrm{NO}_{3}$ is known to have a high electron affinity and should therefore have an influence on the individual cluster charges. Table $7 . \mathbb{1}$ summarizes the Bader charges obtained for the whole

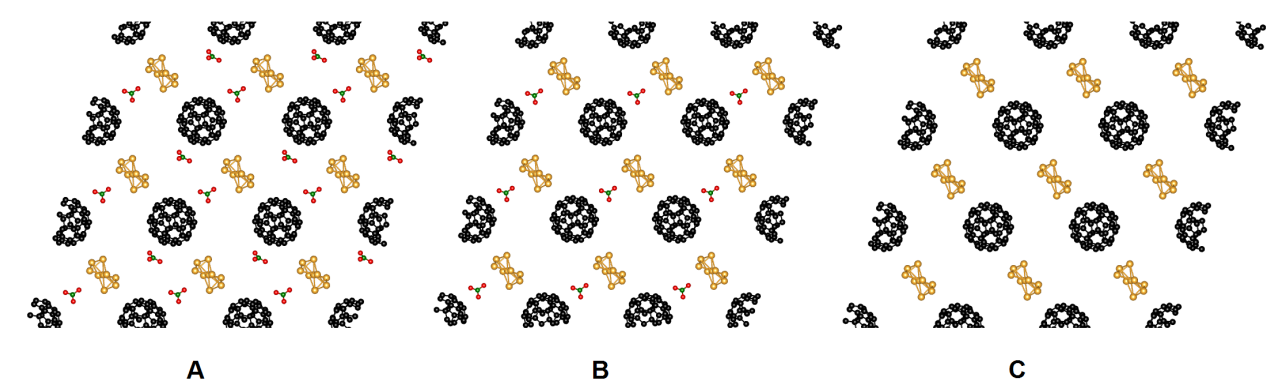

Figure 7.1: Visualization of the theoretical rock-salt type structures obtained after full relaxation. A: binary mixture of $\mathrm{Au}_{9}\left(\mathrm{PPh}_{3}\right)_{8}$ and $\mathrm{C}_{60}$ incorporating $2 \mathrm{NO}_{3}$ molecules, B: exhibiting $1 \mathrm{NO}_{3}$ molecules and $\mathrm{C}$ : without $\mathrm{NO}_{3}$ molecules present. The ligands have been neglected for simplification of the visualization. 
$\mathrm{Au}_{9}\left(\mathrm{PPh}_{3}\right)_{8}$ cluster $q_{\text {totalcluster }}$, the Bader charge of the ligands of the cluster $q_{\text {lig }}$, the gold core of the cluster $q_{\text {core }}$ and the $\mathrm{C}_{60}$ in the structures $\mathrm{A}, \mathrm{B}$ and $\mathrm{C}$. The values in

Table 7.1: Bader charges in e calculated for $\mathrm{Au}_{9}\left(\mathrm{PPh}_{3}\right)_{8}$ (qtotalcluster), the ligands of the cluster qlig, the gold core of the cluster qcore and the $\mathrm{C}_{60}$ in the different compounds $\mathrm{A}, \mathrm{B}$ and $\mathrm{C}$ containing different amounts of $\mathrm{NO}_{3}$. In addition a calculation for system $\mathrm{A}$ including Grimme's dispersion correction ${ }^{[134]}(\mathrm{A}+\mathrm{D} 3)$ can be found.

\begin{tabular}{cccccc}
\hline \hline compound & qtotalcluster & qlig & qcore & qful & $\mathrm{E}_{\text {coh }[\mathrm{eV}]}$ \\
\hline $\mathrm{A}$ & 2.63 & 3.62 & -0.99 & -0.84 & -11.15 \\
$\mathrm{~B}$ & 1.79 & 3.03 & -1.24 & -0.92 & -8.93 \\
$\mathrm{C}$ & 1.11 & 2.60 & -1.48 & -1.11 & -7.18 \\
$\mathrm{~A}+\mathrm{D} 3$ & 2.50 & 3.45 & -0.95 & -0.78 & \\
\hline \hline
\end{tabular}

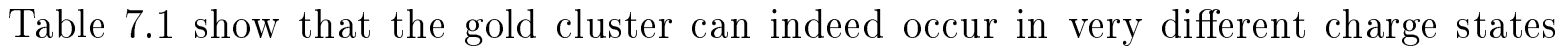
depending on the amount of electron withdrawing units incorporated $\left(\mathrm{NO}_{3}\right.$ molecules). The Bader charges of the gold cluster range form 1.11 e to $2.63 \mathrm{e}$. In addition it can be seen that the ligand charges are more affected then the charges of the gold cores. This is in good agreement with strong coupling between the ligands and the superatomic states as mentioned in the previous chapters. Moreover it can be observed that the charge located at the fullerenes is $\sim 1$ e in all environments. Thus the fullerenes are likely to be found approximately singly charged.

The charges found in structures A and B both very well correspond to the expectation of the crystalline material to consist of a $\mathrm{Au}_{9}\left(\mathrm{PPh}_{3}\right)_{8}^{+2}$ cluster and more positive $\mathrm{C}_{60}^{-}$. Since Bader charges are known to overestimate that actual charge transfer in several cases, model system A seems to give the best agreement with the expectations arising from experiment. In order to include dispersion effects and to compensate for the overestimation of lattice parameters arising from PBE calculations, system A has been additionally computed including the dispersion correction proposed by Grimme. The Bader charges only change in a small range giving the computed values more validity.

Beyond the Bader charges Table $7 . \mathbb{l}$ lists the binding energies calculated as the difference between the total energy of the compound and the energies of each of the two to four fragments in their relaxed form. It can be seen that the binding energy is increasing with the number of $\mathrm{NO}_{3}$ molecules incorporated. Thus, compound A reveals the largest binding energy. 
Due to good agreement with experimentally obtained charges and a relatively large theoretical binding energy, the XRD spectrum of model system A has been calculated and compared to experimental data (GI-XRD). The compound has been found to reveal to much impurity such that the experimentally measured XRD spectrum has been neglected. In this particular case, the PBE calculated structure which does not account for dispersion has been chosen due to the fact that the measurements have been undertaken at room temperature. The fullerene is known to strongly vibrate at high temperatures, thus an overestimation of the lattice parameters of a calculation conducted at $0 \mathrm{~K}$ should be more consistent with experimental results. Figure $\mathbb{7 2}$ shows the experimentally mea-

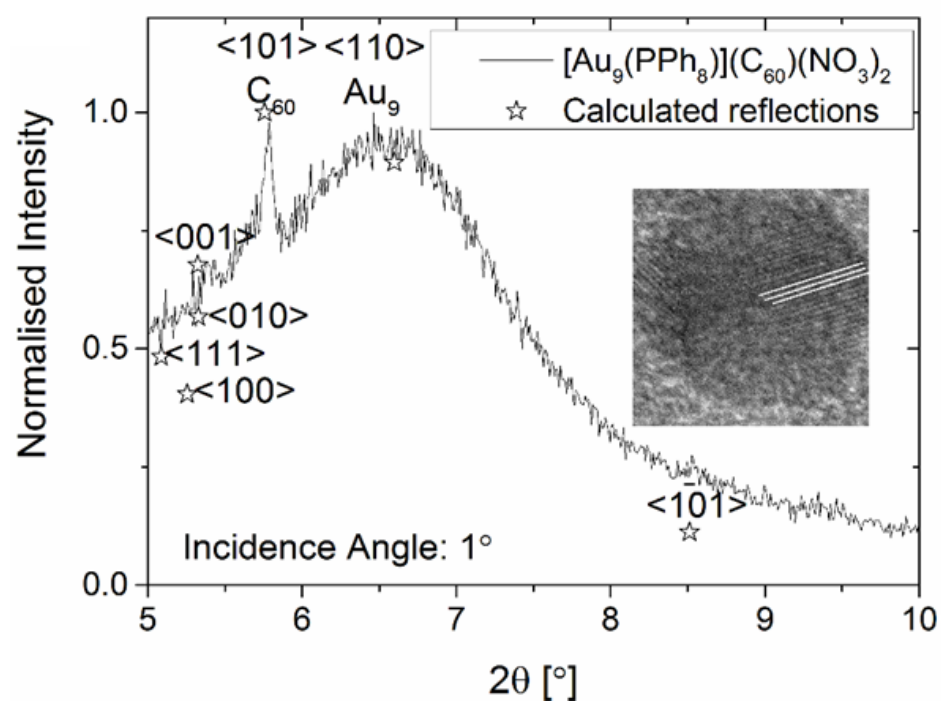

Figure 7.2: Visualization of the experimentally measured GI-XRD spectrum and the computationally obtained main XRD bands for system A indicated with stars. The data is taken from personal communication with Holger Fiedler.

sured GI-XRD spectrum and the main bands of the calculation indicated with stars. And again good agreement between experiment and theoretical results is obtained, suggesting that the actual crystal might indeed depict some similarity to a simple rock-salt structure. Nevertheless, it is expected that deviations arise due to defects in the crystal. Thus, the XRD spectrum was found not to be comparable with the calculations, solely the GI-XRD showed good agreement.

Beyond the atomic structure of these systems, the electronic structure has been investigated by calculating the density of states and the band structure for compound A, and 
for comparative reasons also for compound C. In order to show the superatomic character of the theoretical compound, the angular momentum projected density of states in a sphere around the gold cluster core has been computed and visualized in Fig. 7.3. Mollenhauer et al. have shown that the energetic ordering of the superatomic states in

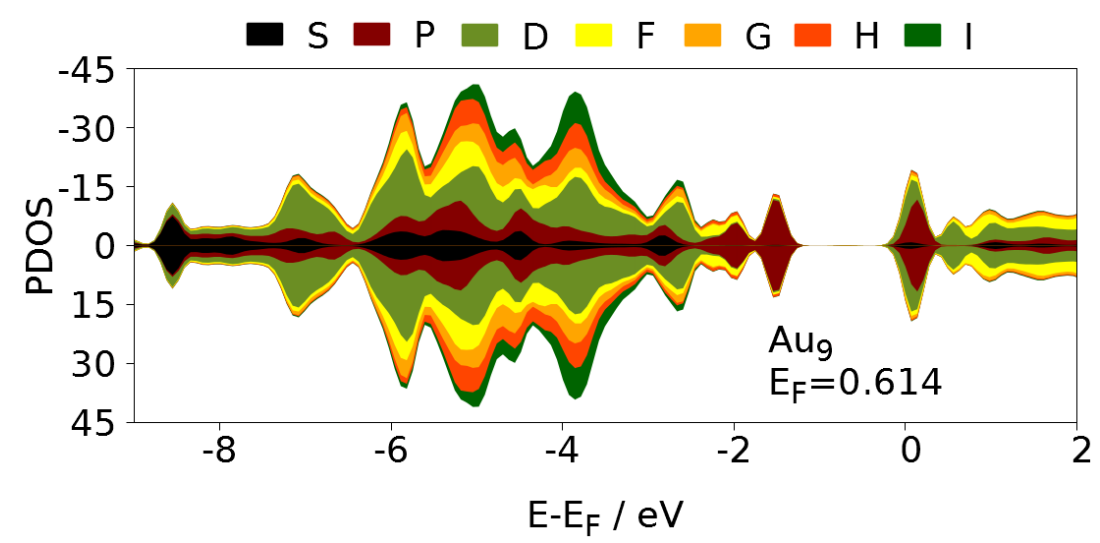

Figure 7.3: Visualization of PDOS in a sphere around the Aug cluster core in compound C.

the phosphine protected $\mathrm{Au}_{9}$ cluster is $\mathrm{S}^{2} \mathrm{P}^{4}$ while the third $\mathrm{P}$ orbital and the first $\mathrm{D}$ or-

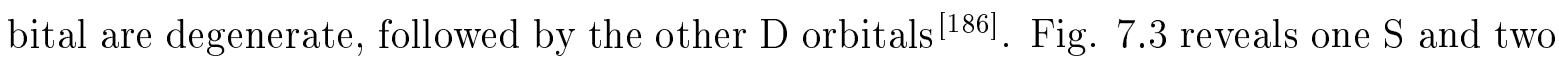
P states below the Fermi level and a more or less degenerate D and P state close to the Fermi energy. This on one hand indicates that three states are fully occupied and that the following state is shifted close to the Fermi level showing the charge of the cluster to be +3 with a tendency to a slightly lower charge, since the uncharged cluster would exhibit nine superatomic electrons. This is in good agreement with the Bader charges of the whole cluster, including ligands, which again indicates a strong involvement of the ligands. Furthermore the Figure reveals that the transferred electrons indeed come from the superatomic states of the gold cluster indicating a superatomic character of the compound. Fig. 7.4 depicts the obtained band structure for a: compound $\mathrm{C}$ and b: compound A. The bands close to the Fermi level are all relatively flat implying high ionic character of the compound. The idea of an ionic compound has been strengthened through the results obtained by an EDA analysis of the nearest neighbor dimer of compound $\mathrm{C}$. The electrostatic interaction between the $\mathrm{C}_{60}$ and the gold cluster has been found to contribute $-1.06 \mathrm{eV}$ to the interaction energy, while the orbital interaction was found to be much smaller $(-0.25 \mathrm{eV})$. Nevertheless, since the HOMO orbital is centered 


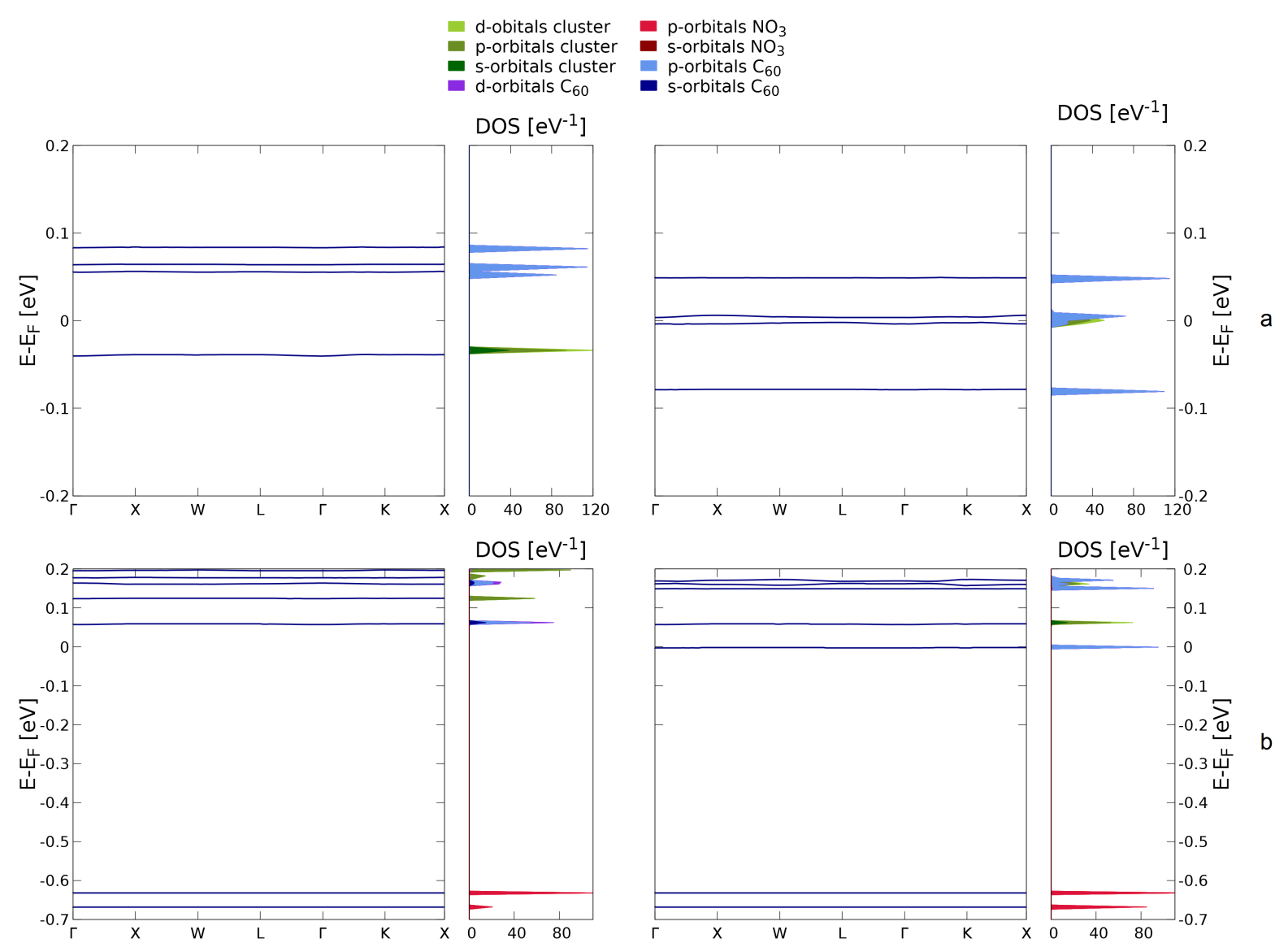

Figure 7.4: Visualization of the band structure for the majority and minority spin of a: compound $\mathrm{C}$, b: compound $\mathrm{A}$. 
at the cation and the calculated orbital contribution to the interaction energy show, that the interaction between both clusters is not of pure ionic nature. Furthermore the band gap has been found to be around 6-50 meV depending on the spin channel.

The whole situation changes when including two $\mathrm{NO}_{3}$ molecules. The bands are still found to be relatively flat but additional states centered at the $\mathrm{NO}_{3}$ molecules close to the Fermi energy appear. The incorporation of the new molecules leads to a widening of the gap. The smallest gap is found to be $\sim 60 \mathrm{meV}$ with the highest occupied band being centered at the fullerene indicating strong ionic nature. The other spin channel reveals a gap of around $700 \mathrm{meV}$ with the $\mathrm{HOMO}$ state centered at the anionic $\mathrm{NO}_{3}$ units. Thus the incorporation of the $\mathrm{NO}_{3}$ molecules seems to widen the band gap and the ionic character of the compound.

\subsubsection{Conclusion}

Holger Fiedler et al. managed to synthesize a compound based on $\mathrm{Au}_{9}\left(\mathrm{PPh}_{3}\right)_{8}$ and $\mathrm{C}_{60}$. Measurements and the computation of $\mathrm{UV} / \mathrm{V}$ is spectra showed that the buckyball is most likely found to be $0-1$ e charged while the charge of the gold cluster is found to be $+2-+3$. In order to determine a potential structure of the compound and analyze the electronic properties three rock-salt type model systems have been constructed incorporating different numbers of $\mathrm{NO}_{3}$ molecules. The calculated XRD spectrum for model compound A was found to be quite similar to the measured GI-XRD spectrum. Furthermore the Bader charges of compound A where found to agree with the expectations arising from experiment. Both findings suggest that the synthesized compound might reveal structural similarity to model compound A.

Beyond that the angular momentum projected density of states depicts the superatomic character of the compound by showing that the electrons transferred between the clusters come from the superatomic charge of gold cluster. The computation of the band structure and EDA analysis of compound $\mathrm{C}$ additionally shows that the model compound reveals strong ionic character, which increases with the incorporation of $\mathrm{NO}_{3}$ molecules. Moreover, the band gap was found to be very small in the case of compound $\mathrm{C}$ and increases in the case of compound A. 


\subsection{Investigation of the Interaction Between Aluminum Oxide Fluoride Clusters and Water}

\subsubsection{Introduction}

Metal oxides are of particular interest for a large variety of applications such as, sensing, as electronic materials and for environmental purposes. One of the key applications however is catalysis. Metal oxides depict the central component in many synthetic conversions. Due to the profound catalytic role of this material class there is an ongoing search for new structures.

In this particular field metal (hydr)oxofluorides used to play a minor role. This mainly changed in 2003 when Kemnitz et al. introduced the so called "fluorolytic sol-gel synthesis" that gave access to previously inaccessible metal fluorides and metal (hydr)oxofluorides, that are not only of catalytic interest, but serve as precursor for the synthesis of various nanostructures ${ }^{[103-[105]}$. On the basis of this interesting finding, the Paulus group theoretically investigated the $\mathrm{Al}_{4} \mathrm{~F}_{4}\left(\mu_{4}-\mathrm{O}\right)\left(\mu-\mathrm{O}^{i} \mathrm{Pr}\right)_{5}\left[\mathrm{H}\left(\mathrm{O}^{i} \mathrm{Pr}\right)_{2}\right]$ (see Fig. 7.5. C) with respect to the degree of fluorination and the position of the fluorine atoms and thus, confirmed that the experimentally found cluster is equivalent to the energetically most stable structure regarding the fluorination. The work presented in the following passages is based on the observations made by both research groups and has been conducted in collaboration with the group of Prof. Paulus. To subsequently gain a better fundamental understanding a new species of hydrophilic (here hydroxylated clusters) aluminum oxide fluoride clusters has been investigated. The iso-propoxide groups protecting the aluminum oxide flouride cluster $\left(\mathrm{Al}_{4} \mathrm{~F}_{4}\right)$ have been replaced by hydroxyl groups (see Fig. 7.5.A). The reaction has been studied by computing reaction free energies and the kinetics of the reaction. Moreover NMR spectra have been calculated to compare the clusters with different substituents. In a next step the role of the fluorine atoms of the hydrophilic $\mathrm{Al}_{4} \mathrm{~F}_{4}$ cluster (see Fig. 7.5. A) in an aqueous medium has been tested by studying the incremental addition of water molecules to the system and performing structure relaxation. The whole process has been repeated for the fully hydroxylated aluminum oxide cluster (see Fig. 7.5.B). 

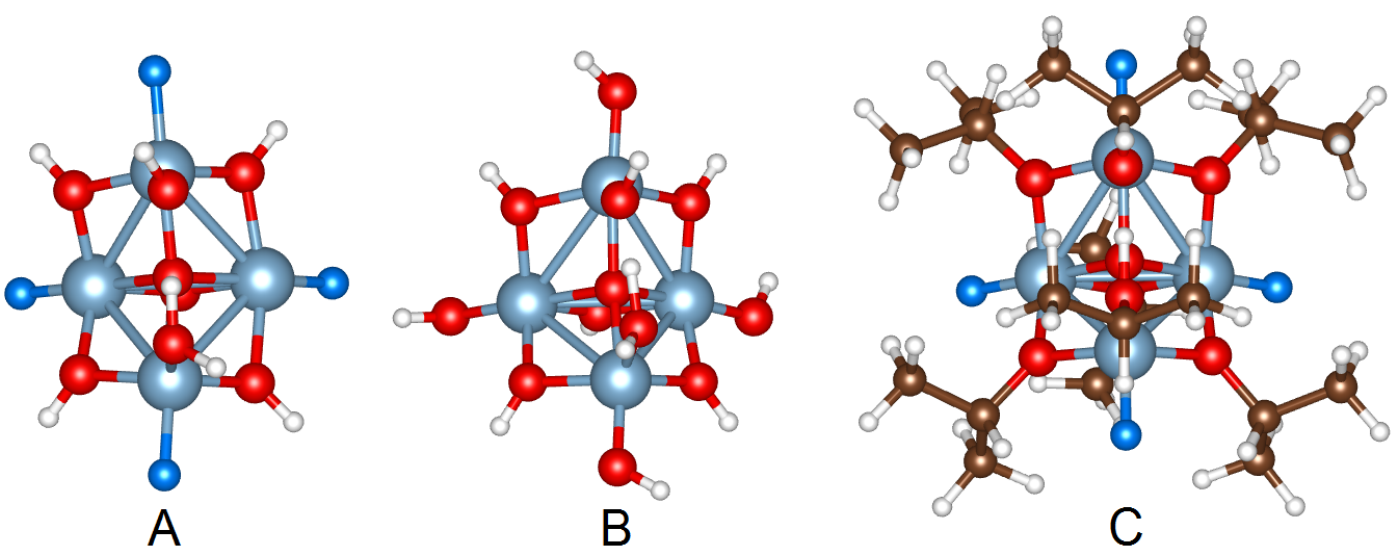

Figure 7.5: Visualization of the investigated aluminum oxide clusters with different substituents. A: $\quad \mathrm{Al}_{4} \mathrm{~F}_{4}\left(\mu_{4}-\mathrm{O}\right)(\mu-\mathrm{OH})_{5}\left[\mathrm{H}(\mathrm{OH})_{2}\right], \quad \mathrm{B}: \quad \mathrm{Al}_{4}\left(\mu_{4}-\mathrm{O}\right)(\mu-$ $\mathrm{OH})_{5}\left[\mathrm{H}(\mathrm{OH})_{2}(\mathrm{OH})_{4}\right], \quad \mathrm{C}: \mathrm{Al}_{4} \mathrm{~F}_{4}\left(\mu_{4}-\mathrm{O}\right)\left(\mu-\mathrm{O}^{i} \mathrm{Pr}\right)_{5}\left[\mathrm{H}\left(\mathrm{O}^{i} \mathrm{Pr}\right)_{2}\right]$. Color code: $\mathrm{Al}$ : gray, F: blue, O: red, C: brown, H: white.

In contrary to the other chapters of this thesis, this project will discuss non-superatomic clusters, due to their strong ionic nature. The gold clusters analyzed in chapter 3 clearly reveal superatomic character while the transition metal chalcogen clusters show an intermediate case with the clusters losing part of their superatomic nature due to charge transfer. This effect is even stronger in the aluminum oxide clusters investigated in the following text passages, leading to a loss of the superatomic nature.

\subsubsection{Methods}

The DFT calculations presented in the following section have all been conducted utilizing the program package Turbomole 7.0.1. In all cases (except for NMR calculations) the meta-GGA functional TPSS [ख0] has been used and the D3 dispersion correction by Grimme et. al ${ }^{[134]}$ with Becke-Johnson damping ${ }^{[134]}$ has been applied. Furthermore a triple zeta valence basis set developed by Ahlrichs et al. was chosen. In order to achieve higher accuracy the radial grid size has been increased to 4 in all cases while the efficiency of the calculations has been increased using the resolution of identity approximation [एँ5] . To analyze the distortion of the clusters in different media COSMO-RS [201,2012] calculations have been performed at $25^{\circ} \mathrm{C}$. Subsequent to the structure relaxations of the $\mathrm{Al}_{4} \mathrm{~F}_{4}$ clusters, with and without utilizing the solvent model COSMO-RS, numeric frequency 
analysis has been performed where the SCF energy convergency criterion has been increased to $10^{-8}$ a.u. Moreover the corresponding NMR spectra have been calculated using the Turbomole default settings (grid: 3, functional: BP86 [203,2014], basis set: def$\mathrm{SV}(\mathrm{P}))$ since it accelerates the calculations while having a minimal impact on the results. The water binding energies $E_{\text {bind }}$ presented, have been obtained as the difference of the total energy of the whole system $E_{\text {sys }}$ (relaxed structure of cluster with all added $\mathrm{H} 2 \mathrm{O}$ ) and the energy of the relaxed system without the additional water molecule $E_{\text {sys }-\mathrm{H}_{2} \mathrm{O}}$ and the total energy of the free, relaxed $\mathrm{H}_{2} \mathrm{O} E_{\mathrm{H}_{2} \mathrm{O}}, E_{\text {bind }}=E_{\mathrm{sys}}-E_{\mathrm{sys}-\mathrm{H}_{2} \mathrm{O}}-E_{\mathrm{H}_{2} \mathrm{O}}$. The lowest binding energy found in each scan has been corrected for the Basis Set superposition Error (BSSE) using the Counterpoise method.

In addition, the reaction path sampling has been performed using the Nudged elastic band method, generating 14-18 pictures (including the reactants and products) [143, [44]. The obtained transition states (TS) have been further optimized and verified by utilizing frequency analysis.

\subsubsection{Discussion}

\section{Reaction}

In a first step the gradual reaction from cluster $\mathrm{C}$ to cluster $\mathrm{A}$ and iso-propanol $\left(\mathrm{Al}_{4} \mathrm{~F}_{4}\left(\mu_{4}-\mathrm{O}\right)\left(\mu-\mathrm{O}^{i} \mathrm{Pr}\right)_{5}\left[\mathrm{H}\left(\mathrm{O}^{i} \mathrm{Pr}\right)_{2}\right]+7 \mathrm{H} 2 \mathrm{O} \rightarrow \mathrm{Al}_{4} \mathrm{~F}_{4}\left(\mu_{4}-\mathrm{O}\right)(\mu-\mathrm{OH})_{5}\left[\mathrm{H}(\mathrm{OH})_{2}\right]+7 \mathrm{HO}^{i} \mathrm{Pr}\right)$ has been investigated calculating the reaction free energies $\Delta E$ for the replacement of one iso-propoxide group with an hydroxyl group as follows, $\Delta E=E_{\text {products }}-E_{\text {reactants }}$, whereby each fragment has been considered in its relaxed form. Two different structural iso-propoxide groups are present in cluster A, 2 terminal groups (label 1 and 2 in Fig. 7.6]) and 5 bridging groups (label 3-7 in Fig. 7.6).

The values in Table 7.2 reveal that the substitution reaction for cluster $\mathrm{C}$ is unfavorable in all cases, while replacing one of the bridging hydroxyl groups is less favorable then replacing a singly bound $\mathrm{OH}$ group. Overall the reaction free energies calculated for cluster $\mathrm{C}$ all lie in the same range. Thus, according to those values, in equilibrium all products are similarly likely to occur. Furthermore, two paths chosen to replace each of the iso-propoxide groups with hydroxyl groups show that the reaction occurring on one 


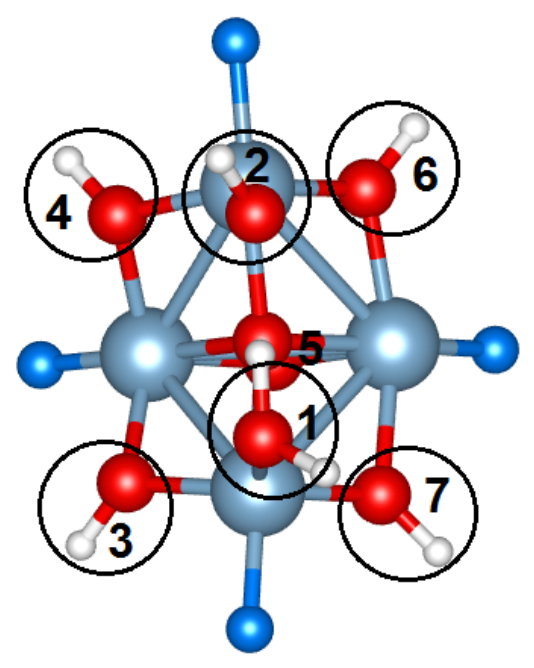

Figure 7.6: Visualization of the different substitution sites in cluster A. The numbers are consistent with the numbering of positions in Table $\mathbf{Z 2}$. Color code: Al: gray, F: blue, O: red, H: white.

Table 7.2: Computed reaction free energies for the reaction of cluster $\mathrm{C}$ or $\mathrm{D}$ with water yielding cluster $\mathrm{C}$ or $\mathrm{D}$ with one iso-propoxide group replaced by a hydroxide group and a iso-propanol. The letters " $\mathrm{t}$ " and "b" indicate the position of the regarded isopropoxide group (terminal or bridged).

\begin{tabular}{cccc}
\hline & & cluster C & cluster D \\
position & No. of position & $\Delta E[\mathrm{~kJ} / \mathrm{mol}]$ & $\Delta E[\mathrm{~kJ} / \mathrm{mol}]$ \\
\hline $\mathrm{t}$ & 1 & 7.16 & -19.97 \\
$\mathrm{t}$ & 2 & 6.27 & -15.01 \\
$\mathrm{~b}$ & 3 & 10.73 & -28.69 \\
$\mathrm{~b}$ & 4 & 10.46 & -26.66 \\
$\mathrm{~b}$ & 5 & 14.81 & -1.14 \\
$\mathrm{~b}$ & 6 & 13.08 & -19.79 \\
$\mathrm{~b}$ & 7 & 13.10 & -11.45
\end{tabular}


Table 7.3: Reaction free energies for the stepwise transformation of cluster $\mathrm{C}$ to cluster A along the minimal energy at each substitution step (path 1) and along the maximum energy path 2 .

\begin{tabular}{cccccc}
\hline & \multicolumn{2}{c}{ path 1} & \multicolumn{3}{c}{ path 2} \\
No. substitution reaction & No. of position & $\Delta H[\mathrm{~kJ} / \mathrm{mol}]$ & No. of position & $\Delta H[\mathrm{~kJ} / \mathrm{mol}]$ \\
\hline 1 & 2 & 6.27 & 5 & 14.81 \\
2 & 4 & 9.83 & 7 & 12.25 \\
3 & 3 & 10.08 & 4 & 12.14 \\
4 & 6 & 12.09 & 6 & 11.52 \\
5 & 1 & 12.38 & 3 & 11.88 \\
6 & 7 & 12.82 & 1 & 5.85 \\
7 & 5 & 14.48 & 2 & 10.5
\end{tabular}

site of the cluster has little influence on the reaction free energy of the second reaction and so on (see Table 7.3 ). The reaction free energy values are only slightly affected, which shows that also the second, third etc. reaction appears to happen at random sites. Besides $\Delta E$ for the reaction of cluster $\mathrm{C}$ with one water molecule, Table 7.2 lists similar values for the substitution reaction at the fully iso-propoxylated cluster D visualized in Fig. 7.7. In contrast to cluster $\mathrm{C}$ all substitution reactions at sites 1-7 regarding cluster D are favorable, which can be explained by steric effects. The bulky iso-propoxide groups sterically hinder each other, thus the substitution with smaller groups is energetically favorable. Another difference to cluster $\mathrm{C}$ is that the reaction site seems to play a more significant role. According to the reaction free energy the reaction will occur preferably at the site that experiences the largest steric effect, namely site 3 and 4. At this positions the steric effect, comparing cluster $\mathrm{C}$ with $\mathrm{D}$, is found to be nearly $40 \mathrm{~kJ} / \mathrm{mol}$. Due to the high symmetry of the aluminum oxide structure, the second highest effect can be found for positions 6 and 7, while the third highest value of $\sim 20 \mathrm{~kJ} / \mathrm{mol}$ is found for positions 1 and 2 . The lowest effect, thus the smallest exothermic contribution is found for the iso-propoxide group located at the back of the cluster in Fig. 7.7 .

It has to be considered that $\Delta E$ solely give a first idea about the reaction. This reduces computational costs, and as proven in the former study shows that the reaction free energies are only slightly affected by the previous substitution. The bulky ligands of cluster D shielding the nanoparticle from approaching water molecules and other effects 


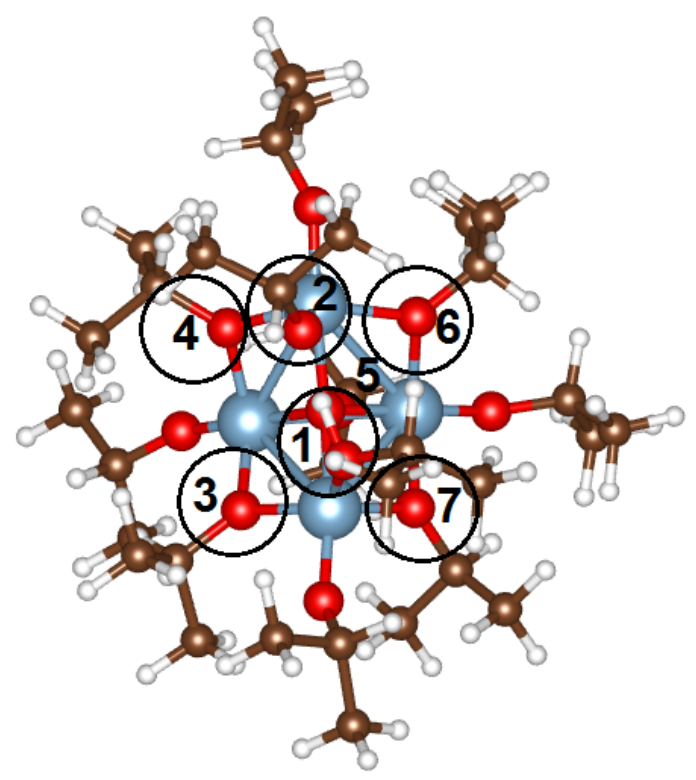

Figure 7.7: Visualization of the aluminum oxide cluster D: $\mathrm{Al}_{4}\left(\mu_{4}-\mathrm{O}\right)\left(\mu-\mathrm{O}^{i} \mathrm{Pr}\right)_{5}\left[\mathrm{H}\left(\mathrm{O}^{i} \mathrm{Pr}\right)_{6}\right]$. The numbers of the $\mathrm{O}^{i} \mathrm{Pr}$ groups are consistent with the numbering of positions in Table $\mathbf{7 . 2}$ Color code: Al: gray, O: red, C: brown, H: white.

have not been considered.

Since the one of the main proposes of this study is to obtain and study the hydrophilic cluster B, cluster $\mathrm{C}$ has been taken as starting point for the study of the hydroxylation reaction.

To investigate the kinetics of the replacement reaction, only the substitution of the last bridging and the last terminal iso-propoxide group has been considered. Fig. 7.8 visualizes the reaction taking place at a singly bonded iso-propoxide group. Starting from an adsorbed water molecule in the most stable adsorption site in vicinity of the $\mathrm{O}^{i} \operatorname{Pr}$ group, as visualized in 7.8 . a the reaction proceeds with an adsorption of the water molecule at the aluminum atom the iso-propoxide group is bound to. This is followed by a hydrogen transfer. In a next step a breaking of the bond between the aluminum atom and the hydrogenated iso-propoxide group can be observed yielding an iso-propanol and cluster A. The reaction barrier between the initial position of the reactants and the water molecule being adsorbed at the aluminum atom is about half the size of the main barrier. Thus, the major barrier of $61.43 \mathrm{~kJ} / \mathrm{mol}$ has been calculated for the whole reaction with 


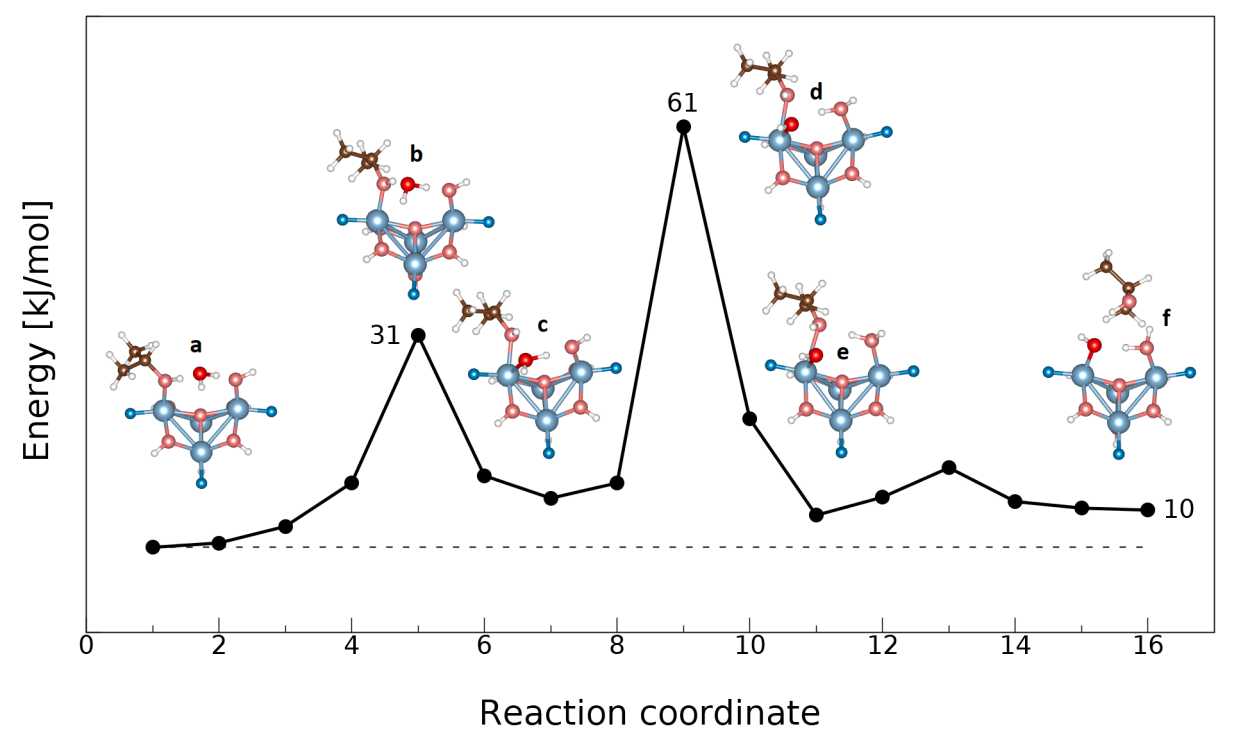

Figure 7.8: Reaction path for the reaction of $\mathrm{Al}_{4} \mathrm{~F}_{4}\left(\mu_{4}-\mathrm{O}\right)(\mu-\mathrm{OH})_{5}\left(\mathrm{O}^{i} \mathrm{Pr}\right)\left(\mathrm{H}_{2} \mathrm{O}\right)$ with a water molecule yielding iso-propanol and cluster B. Additionally the structures of selected NEB point are visualized (see a-f). The numbers within the picture indicate selected energies relative to the initial structure in $\mathrm{kJ} / \mathrm{mol}$. Color code: Al: gray, F: blue, O: red, C: brown, H: white. 
Fig. 7.8 .d being the main transition state. The reaction has been found to be slightly endothermic with $\Delta E_{a}=5.44 \mathrm{~kJ} / \mathrm{mol}$. The reaction rate at different temperatures can be estimated using the Eyring equation [005],

$$
k(T)=\frac{k_{B} T}{h} e^{-\frac{\Delta E_{R}^{\ddagger}(T)}{R T}},
$$

$k$ is the reaction rate, $k_{B}$ the Boltzmann constant, $h$ the Planck constant, $T$ the temperature, $R$ the universal gas constant and $\Delta E_{a}^{\ddagger}$ the activation barrier calculated as the difference between the energy of the reactant and the transition state. At room temperature $(298.15 \mathrm{~K})$ the reaction rate is approximately $1.01 \times 10^{2} \mathrm{~s}^{-1}$. Since the calculated rate indicates a slow conversion, the reaction can be accelerated by e.g. increasing the temperature. Fig. $[7.8$ visualizes the hydroxylation at a bridging iso-propoxide group.

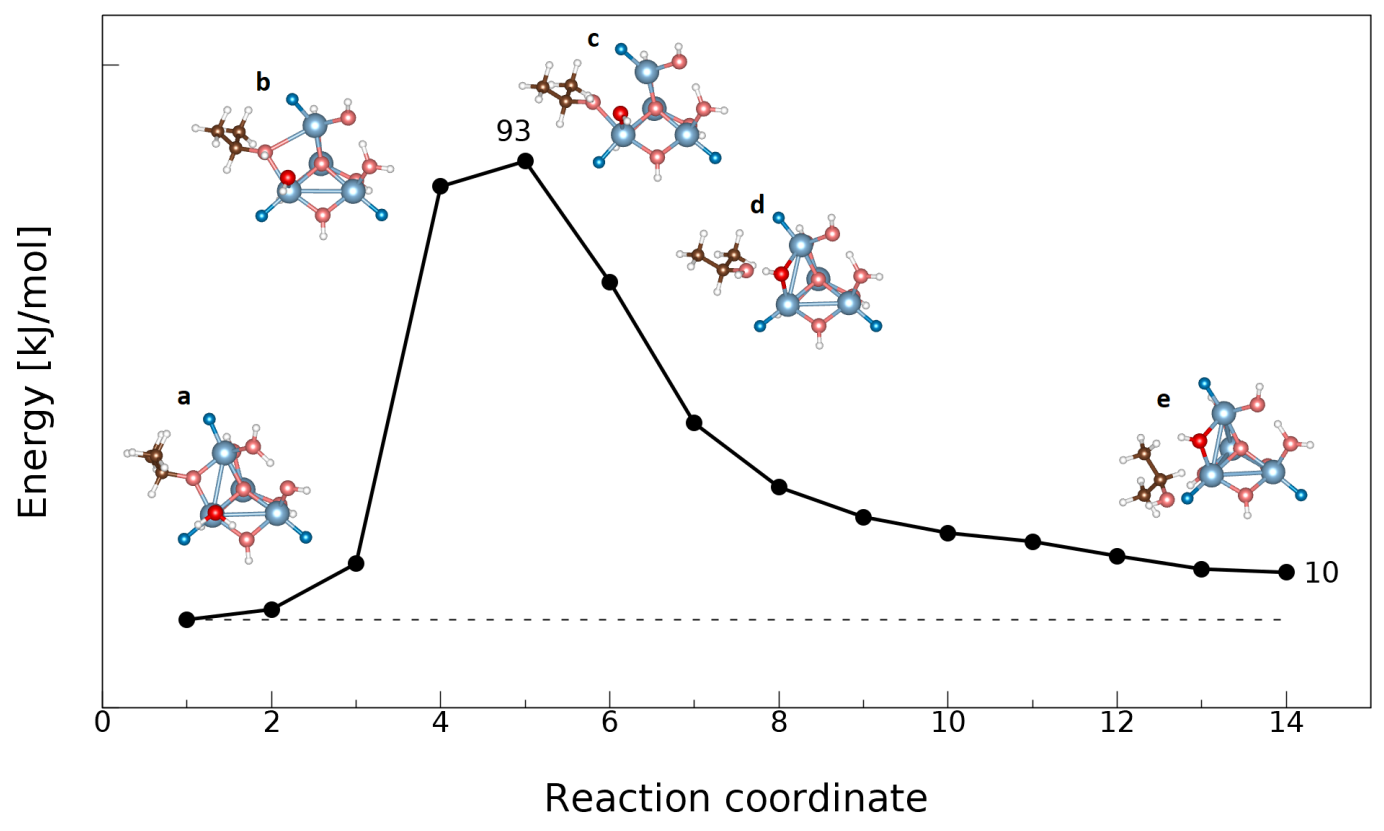

Figure 7.9: w

ith $\left.\mathrm{H}_{2} \mathrm{O}\right]$ Reaction path for the reaction of $\mathrm{Al}_{4} \mathrm{~F}_{4}\left(\mu_{4}-\mathrm{O}\right)(\mu-\mathrm{OH})_{4}\left(\mu-\mathrm{O}^{i} \mathrm{Pr}\right)_{1}\left[\mathrm{H}(\mathrm{OH})_{2}\right]$ with a water molecule yielding iso-propanol and cluster B. Additionally the structures of selected NEB point are visualized (see a-e). The numbers within the picture indicate selected energies relative to the initial structure in $\mathrm{kJ} / \mathrm{mol}$. Color code: $\mathrm{Al}$ : gray, $\mathrm{F}$ : blue, $\mathrm{O}$ : red, $\mathrm{C}$ : brown, $\mathrm{H}$ : white. 
The water molecule is initially adsorbed at one of the aluminum atoms the $i s o$-propoxide group is bound to, which represents a stable position in vicinity of the $\mathrm{O}^{i} \mathrm{Pr}$ group. The reaction starts with a hydrogen transfer between the water molecule and the oxygen atom of the iso-propoxide group leading to an opening of the bridge and the release of iso-propanol while a new bridged hydroxyl group is formed. The reaction barrier has been calculated to be $-90.22 \mathrm{~kJ} / \mathrm{mol}$ with picture $7.8 . \mathrm{c}$ visualizing the transition state. Using eq. 7.7 yields a reaction rate of $6.09 \times 10^{-2} \mathrm{~s}^{-1}$, which shows that the reaction is kinetically inhibited at room temperature. Thus, e.g. an increase in temperature or the use of a catalyst is needed to overcome the barrier.

\section{Adsorption of Water Molecules on Clusters A and B in Dependence of the Hydration Degree}

Subsequent to the study of the potential synthesis of cluster A, a water scan has been performed to compare the water adsorption behavior of clusters A and B and to study the influence of the fluorine atoms.

The clusters exhibit many possible water adsorption sites. To get an insight in the stability of the various sites a scan of different starting positions has been performed as follows: The water molecule has been placed at 20 different, equally spread positions around each cluster (see Fig. 7.10) and a full structure relaxation has been performed for each starting position. This has been done for two initial orientations of the water molecule: (1) the oxygen atom pointing towards the cluster, (2) the hydrogen atoms pointing toward the cluster. In the case of cluster $\mathrm{A}$ the nearest $\mathrm{H}_{2} \mathrm{O}$ atom was placed in a distance of $4.5 \AA$ to the central oxygen atom of the cluster, yielding distances of about 1-4 $\AA$ to the nearest cluster atom whereas in the case of cluster $B$ the initial distance to the central atom was chosen to be $5 \AA$ to prevent the water molecule to be placed at the same position where the hydroxyl groups replacing the $\mathrm{F}$ atoms are located. Calculations with new water molecules being placed on top of already existing water molecules have not been considered.

After each scan, the structure with the lowest or highest attractive binding energy has been taken and a next $\mathrm{H}_{2} \mathrm{O}$ molecule has been added performing a scan similar to the first scan. The whole process has been repeated until a first monolayer water shell 


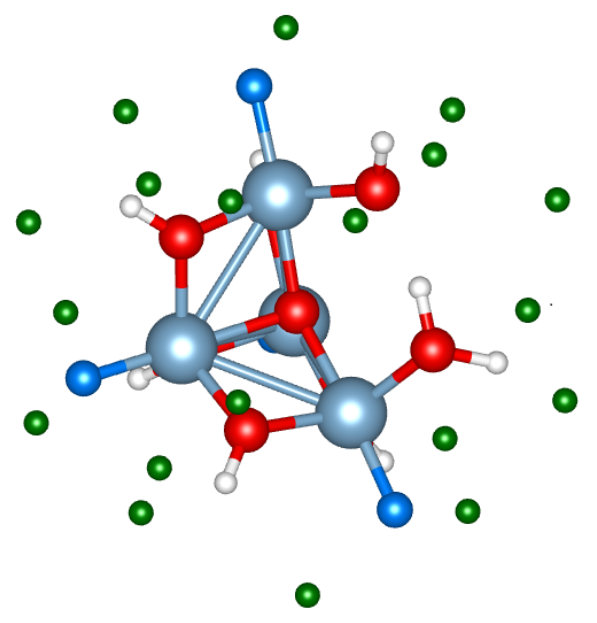

Figure 7.10: Different initial positions of the water molecule for each scan marked in green. Color code: Al: gray, $\mathrm{F}$ : blue, O: red, $\mathrm{H}$ : white.

around each cluster has been obtained (up to $40 \mathrm{H}_{2} \mathrm{O}$ ). Fig. 7.71 and 7.12 show the

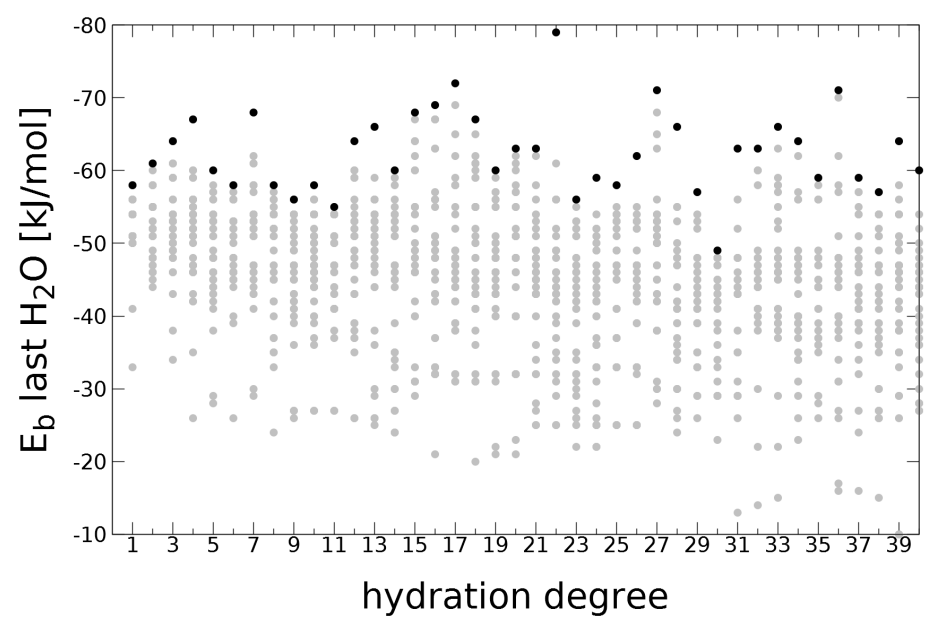

Figure 7.11: Binding energies $E_{\mathrm{B}}$ of the last water molecule added to the system at different initial positions around cluster A. The lowest binding energies obtained in each scan are marked in black.

binding energies $E_{\mathrm{B}}$ for the last $\mathrm{H}_{2} \mathrm{O}$ molecule added for every hydration degree, for clusters A and B respectively. The number "1" at the $\mathrm{x}$-axis indicates the binding energies of the first water molecule adsorbed at the cluster, obtained trough relaxation of the structure with different initial positions of the water molecule etc. All circles 


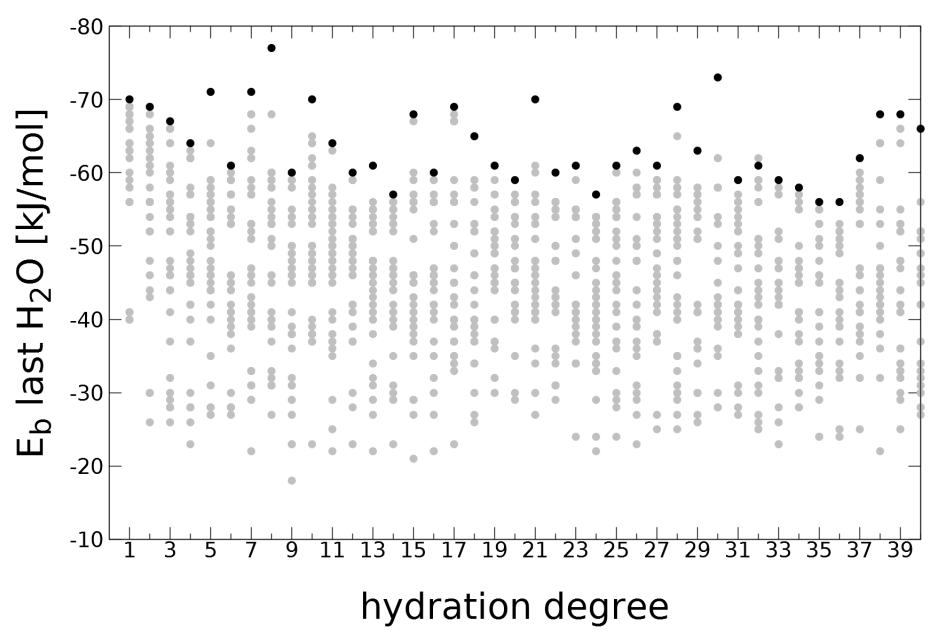

Figure 7.12: Binding energies $E_{\mathrm{B}}$ of the last water molecule added to the system at different initial positions around cluster B. The lowest binding energies obtained in each scan are marked in black.

displayed are converged minimum structures. The lowest binding energies obtained in each scan are indicated with black circles, whereas Fig 7.13 and 7.14 solely show the lowest counterpoise corrected binding energies in the case of both clusters for every scan. In both scans (only considering the lowest binding energies in each scan) very

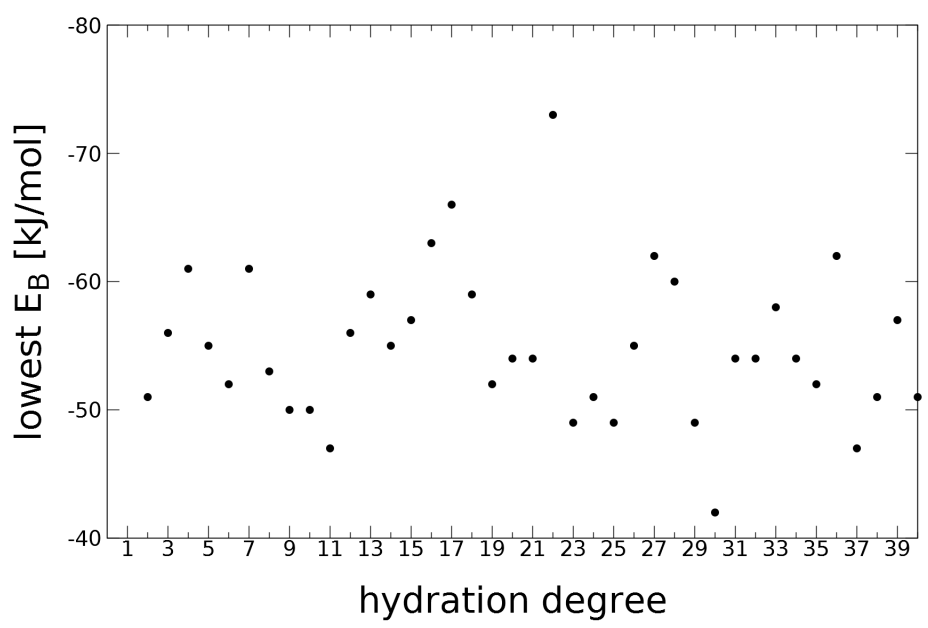

Figure 7.13: Lowest counterpoise corrected binding energies $\mathrm{E}_{B}$ for the last water molecule added to the system in each scan. The scan has been conducted around cluster A. 


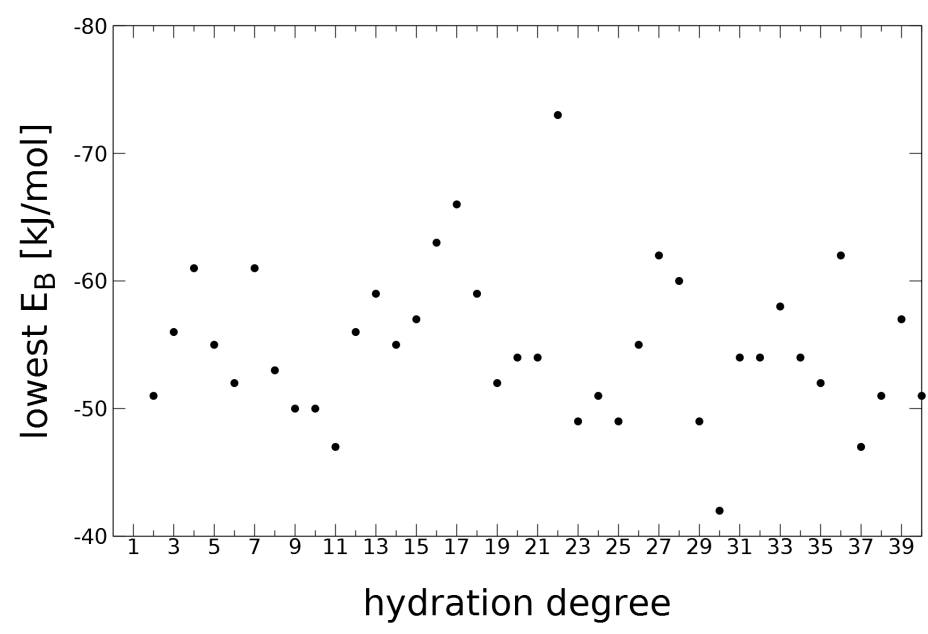

Figure 7.14: Lowest counterpoise corrected binding energies $E_{\mathrm{B}}$ for the last water molecule added to the system in each scan. The scan has been conducted around cluster B.

low binding energies of about $-70--55 \mathrm{~kJ} / \mathrm{mol}$ usually correspond to a situation where an additional water molecule can lead to the formation of around three or four new hydrogen bonds. For medium energies around two new bonds are formed. Relatively high energies are obtained when the added water molecule is solely adsorbed at an aluminum atom. Moreover it is known that one hydrogen bond in water is on average $20 \mathrm{~kJ} / \mathrm{mol}$ strong. Up to four hydrogen bonds per molecule can be formed.

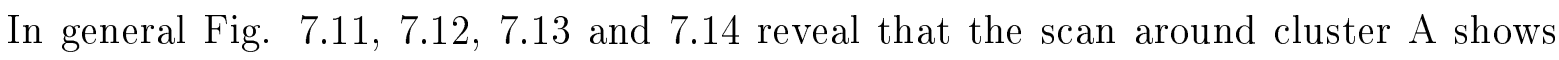
a larger variety of binding energies considering both, all and solely the lowest binding energy in each scan. In addition, the highest and lowest binding energy considering solely the lowest energies in each scan (Fig. 7.13 and 7.14) have been obtained in the case of cluster A. However, taking a closer look at Fig. 7.13 and 7.14 reveals that the water molecules on average bind stronger to cluster B than to cluster A. The average binding energy for cluster A obtained from Fig. 7.73 is $-55.34 \mathrm{~kJ} / \mathrm{mol}$, while the value for cluster B is $-57.29 \mathrm{~kJ} / \mathrm{mol}$.

Another central difference is the way the water molecules are adsorbed. In the case of cluster B the first of the lowest binding energies in each scan is found to be low and slowly increases until four water molecules are added. This corresponds to an adsorption 
of all molecules at different positions. The first four $\mathrm{H}_{2} \mathrm{O}$ molecules do not interact with each other but form hydrogen bonds to the hydroxyl groups of the cluster. Cluster A shows the opposite trend due to the fact that fewer hydroxyl groups are available at the cluster core and an interaction with the fluorine atoms is less favorable. Therefore each added water molecule is located close to another water molecule to interact with. The water molecules start clustering around the aluminum cluster leading to a decrease of the lowest binding energy obtained in each scan with every water molecule added. For cluster B a similar clustering process can be observed starting from the fifth molecule.

In addition, Fig. 7.5 shows that both water molecule monolayers are differently

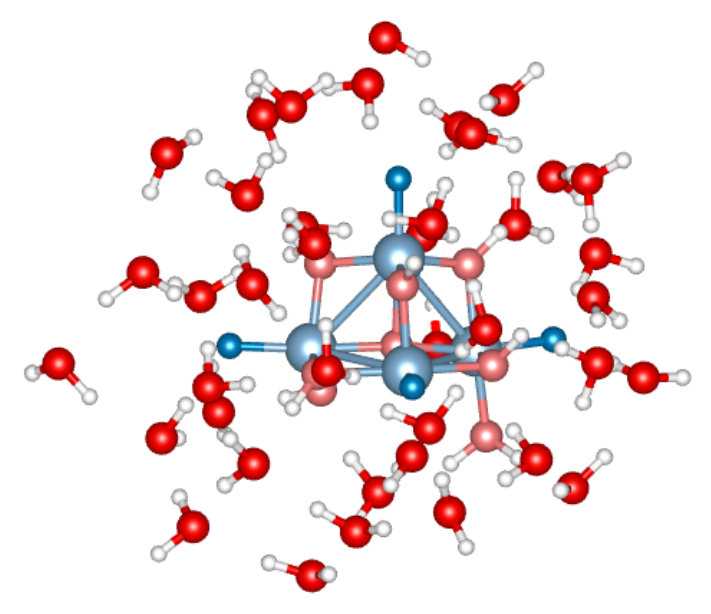

A

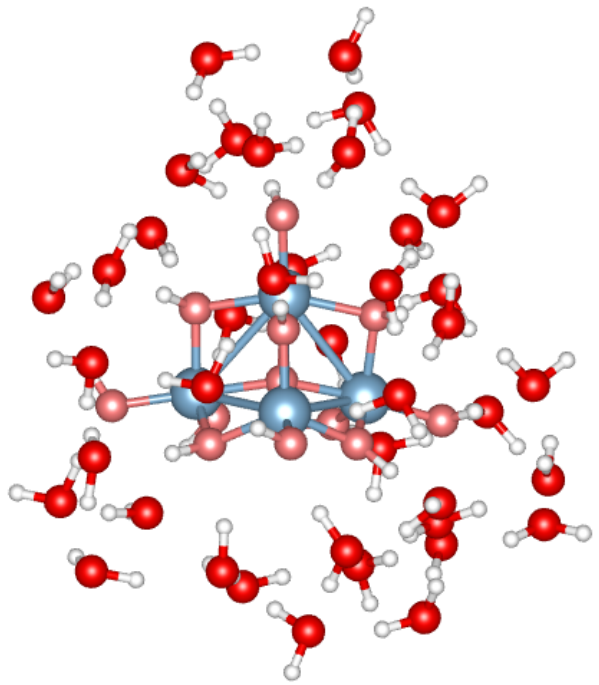

B

Figure 7.15: Visualization of the water shell obtained adsorbing 40 water molecules on cluster A and B. Color code: Al: gray, F: blue, O: red, H: white.

shaped. In the case of cluster A it can be seen that the water molecules interact with the cluster as well as with the other molecules. The 35th molecule added is the first one to lead to a 2nd shell type binding whereby the water molecule only engages with other water molecules from the first shell. The water molecules around cluster B however, show a different type of binding. The water molecules seem to cluster more, thus to interact more with one another. This might be due to the fact that the singly bonded hydroxyl groups protrude further away from the cluster than the single fluorine atoms 
and are therefore keeping the water molecules further away from the cluster itself. This, however, promotes the interaction between the water molecules.

\section{NMR}

NMR chemical shifts for cluster A, B and C in vacuum (Table 17.4) are calculated to investigate an accessible experimental detection tool. To see the influence of the solvent, the shifts for A and B have also been calculated in water using COSMO-RS (with $\epsilon=78.5$ $\left(25^{\circ} \mathrm{C}\right)$ ) and in an additional calculation for the clusters exhibiting a monolayer of 40 water molecules (without the usage of COSMO). Taking a look at the clusters in vacuum, one can see that the biggest difference between the NMR shifts is occurring regarding cluster B and $\mathrm{C}$ which can still be considered a small change. This is simply due to the fact, that the bulkier iso-propoxide groups have little influence on the cluster structure, since they still have enough space to not experience strong repulsive steric effects. In addition, the inclusion of an implicit solvent model also reveals a rather small effect on the NMR shifts, due to small changes in the cluster structure. 
Table 7.4: NMR chemical shifts for the atoms the different clusters have in common with cluster $\mathrm{A}$ and NMR shifts using COSMO with $\mathrm{H}_{2} \mathrm{O}$ as solvent at $25^{\circ} \mathrm{C}$. Furthermore the calculated shifts for cluster $\mathrm{A}$ and $\mathrm{B}$ with a water shell for 40 molecules. Calculated using the following standards: $\left[\mathrm{Al}\left(\mathrm{OH}_{2}\right)_{6}\right]^{2+}, \mathrm{SiMe}_{4}, \mathrm{CCl}_{3} \mathrm{~F}$. The values for cluster $\mathrm{C}$ have been provided by Johannes Horst Budau.

\begin{tabular}{|c|c|c|c|c|c|c|c|}
\hline \multicolumn{8}{|c|}{ shifts in $\mathrm{ppm}$} \\
\hline atoms & cluster A & $\begin{array}{l}\text { COSMO } \\
\text { cluster A }\end{array}$ & $\begin{array}{c}\text { water shell } \\
\text { cluster A }\end{array}$ & cluster B & $\begin{array}{l}\text { COSMO } \\
\text { cluster B }\end{array}$ & $\begin{array}{c}\text { water shell } \\
\text { cluster B }\end{array}$ & cluster $\mathrm{C}^{[06]}$ \\
\hline $\mathrm{Al}$ & 40.7 & 41.7 & 13.1 & 44.9 & 47.0 & 48.0 & 43.7 \\
\hline $\mathrm{Al}$ & 44.0 & 45.3 & 18.7 & 46.5 & 50.5 & 52.3 & 48.2 \\
\hline $\mathrm{Al}$ & 44.0 & 45.3 & 50.5 & 47.8 & 50.5 & 20.3 & 48.1 \\
\hline $\mathrm{Al}$ & 41.7 & 42.79 & 47.1 & 45.8 & 47.6 & 50.2 & 43.4 \\
\hline $\mathrm{F}$ & -190.3 & -188.4 & -178.3 & - & - & - & -173.6 \\
\hline $\mathrm{F}$ & -190.2 & -188.3 & -183.0 & - & - & - & -158.4 \\
\hline $\mathrm{F}$ & -191.0 & -187.3 & -179.1 & - & - & - & -192.7 \\
\hline $\mathrm{F}$ & -192.7 & -191.2 & -191.4 & - & - & - & -218.2 \\
\hline $\mathrm{H}$ & 0.8 & 1.3 & 5.2 & 1.0 & 1.4 & 5.6 & \\
\hline $\mathrm{H}$ & 0.7 & 1.1 & 3.1 & 0.7 & 1.2 & 6.4 & \\
\hline $\mathrm{H}$ & 0.7 & 1.0 & 5.2 & 0.7 & 0.8 & 5.2 & \\
\hline $\mathrm{H}$ & 0.7 & 1.1 & 7.7 & 0.4 & 0.6 & 6.0 & \\
\hline $\mathrm{H}$ & 0.7 & 1.1 & 4.7 & 0.9 & 1.2 & 5.1 & \\
\hline $\mathrm{H}$ & 13.7 & 10.8 & 5.5 & 13.7 & 11.3 & 8.9 & \\
\hline $\mathrm{H}$ & 0.0 & -0.1 & 3.0 & -0.2 & -0.3 & 3.5 & \\
\hline $\mathrm{H}$ & 0.8 & 1.5 & 8.3 & 0.6 & 1.1 & 6.2 & \\
\hline
\end{tabular}

This, however, changes when the solvent is considered explicitly in the form of a single layer of water molecules encapsulating the cluster. The water molecules still have little effect on the aluminum-fluorine distance leading to small changes in the fluorine shifts. The aluminum and hydrogen shifts however, are altered significantly. Treating the solvent explicitly and not as a dielectric continuum allows for effects such as single water molecule adsorption on the aluminum atoms to occur. This has a strong effect on the coordination number of the aluminum atoms, the electron density and therefore the aluminum NMR signals. All aluminum atoms in vacuum are 5-fold coordinated. Including the solvent explicitly however leads to an adsorption of a $\mathrm{H}_{2} \mathrm{O}$ molecule at the first two aluminum atoms of cluster $\mathrm{A}$ and the third aluminum atom of cluster B. Therefore each of these atoms is found to be 6-fold coordinated and its NMR signal is significantly down-shifted.

Furthermore, the water molecules surrounding the cluster form hydrogen bridges to the 
hydroxide groups, which leads to a shift of the hydrogen NMR signals. The shifts of all those $\mathrm{H}$ atoms lie between protons and hydrogens atoms.

\subsubsection{Conclusion}

Investigating the gradual reaction from cluster $\mathrm{C}$ to A has shown that the hydroxylation reaction happening at one site of the cluster has only small influence on the following hydroxylation reaction. This changes due to steric effects, when the fluorine atoms are substituted by bulkier groups.

Moreover, two distinguishable reaction sites have been identified and the kinetics of the reaction, replacing one iso-propoxide group with an hydroxyl group leading to an alcohol and cluster A, have been studied. It has been found that the reaction occurring at a bridged $\mathrm{O}^{i} \mathrm{Pr}$ group is kinetically inhibited at room temperature while the reaction at a singly bonded iso-propoxide group is very slow. The latter reveals a preceding barrier which leads to further decrease of the reaction pace. In both cases it seems to be reasonable to perform the reaction at increased temperatures.

In a second step the difference of the water adsorption behavior of cluster $\mathrm{A}$ and $\mathrm{B}$ has been investigated by performing scans with single water molecules, leading to a monolayer water shell enclosing each cluster. It has been found that the obtained binding energies for cluster A cover a larger variety due to the fluorine atoms offering a different way to bind the water molecules. Nevertheless, cluster B was found to reveal a larger attractive average binding energy. Moreover, in the case of cluster B, the water molecules were found to interact stronger with one another.

Subsequent NMR analysis of the discussed clusters revealed only small structural changes between cluster $\mathrm{A}, \mathrm{B}$ and $\mathrm{C}$. The additional use of a solvent $\left(\mathrm{H}_{2} \mathrm{O}\right)$ in the form of a dielectric continuum did as well not result in major changes. A noticeable difference in the NMR shifts however, has been obtained investigating the clusters enclosed by an explicit water shell. The main reason are effects like the adsorption of water molecules on aluminum atoms and the formation of hydrogen bridges between the hydroxyl groups of the cluster and the surrounding $\mathrm{H}_{2} \mathrm{Os}$. 


\section{Summary}

The work presented in this thesis discusses various factors that influence the application of superatomic clusters as building blocks for new solid state materials. Thus, this study aims to give guidance towards important aspects that need to be considered in order to create designer materials exhibiting specific properties. The icosahedral gold cluster $\mathrm{Au}_{13}$ has been chosen as a model system in order to study characteristics of superatoms which also accounts for other superatomic clusters revealing a straightforward superatomic electron count, such as silver and aluminum clusters.

The structure and characteristics of superatomic materials are even more complex then those of individual atom based solid states materials. Thus, a simple concept entirely predicting the experimental outcome when trying to assemble superatomic clusters can not be established and is furthermore not even known for individual atom based materials. However, important criteria have been identified and discussed in the previous chapters.

One central reason for the use of clusters as bulk building blocks is that the properties of the bulk assemblies can be tuned by solely changing the properties of the clusters involved. Therefore the tunability of the electronic structure of superatomic clusters has been investigated in chapter [3. It was found that already a single atomic impurity can change the electronic situation of a small cluster dramatically while maintaining a superatomic structure. Based on the dopant chosen the gold cluster's electron affinity and electron donor/acceptor behavior can be fine tuned such that it is resembling the EA of individual atoms. Furthermore it was shown that doping can increase the stability of the cluster by increasing the HOMO-LUMO gap. Chapter $\mathbb{t}$ however, discusses clusters that are not tunable and furthermore shows the loss of the superatomic characteristics in strongly ionic clusters which is emphasized in the second experimental collaboration 
(see section 7.2), that analyzes non superatomic ionic clusters. Beyond that chapter 1 reveals that transition metal clusters have a superatomic structure that can only directly be contributed to by the $s$ electrons of the atoms.

A very important factor when thinking of superatomic cluster assemblies are the ligands which are mentioned throughout the whole thesis. It is not only shown that the superatomic orbitals are extended over the ligands, but can also be tuned by the choice of the dopant. This extent of the orbitals can have an positive impact on the clusters to self assemble when thinking of orbital overlap. Moreover chapter $\mathbb{1}$ reveals that the ligands can act as dielectric medium between clusters enabling charge transfer.

The presented work furthermore shows that the stability of the clusters is mostly arising from the stability of the clusters core itself and the ligands binding to the cluster core. Neglecting the ligands leads to coalescence as shown in chapter 5 . The inter cluster ligand-ligand interaction is found to be relatively small and mostly dispersion dominated. This was shown to be an important aspect for assemblies since the ligands need to be flexible and able to interact with the surrounding clusters. This is partially due to the fact that dispersion interactions are found to be one of the main binding contributions to the overall cohesive energy of superatomic cluster based assemblies. In addition chapter 6 exhibits that the choice of the ligands protecting the clusters is able to tune the stability, the electron affinity, the ionization potential and the shape of the superatomic orbitals.

The concepts arising from the first two results chapters where tested in chapter 5 by looking at actual cluster based bulk materials. It was found that similar to individual atom based materials, the size ratio of the used clusters, the electron affinity and the ionization potential play a central role when thinking of the stoichiometric assembly and the charge transfer between clusters. Moreover many similarities to specific atom based solids such as $\mathrm{NaCl}$ have been identified when using clusters revealing similar EA, IP and size ratio as the regarded atomic counterparts.

In addition, it has been shown that even in the case of low dimer interactions high solid state cohesive energies can be achieved. Confining the clusters in a fixed lattice usually leads to smaller distances and therefore larger orbital overlap. The orbital interaction between the clusters has been found to be relatively large in all studied materials while 
the electrostatic interaction was found to dominate in all cases. Both contributions are important considering electronically active materials. The band structures in the context of the EDA analysis also show that the dispersion of the bands is related to the degree of orbital interaction present in the materials or the ratio of the orbital interaction to electrostatic interaction.

In conclusion, the choice of dopants and ligands can be fine tune factors influencing electronic properties and the stability of the overall cluster, as explained above. When considering assemblies the electronic properties such as the EA and IP of the regarding clusters and the size ratio help to predict stable structures and potential electronic properties. Important for the stability and properties are furthermore the orbital and the electrostatic contribution to the interaction energy between the clusters, the binding mode and a large dispersion interaction. A few of these principles such as the size ratio, the dispersion of the bands and the importance of dispersion interaction, have been tested and approved in an experimental collaboration with Fiedler et al. (see section [.].]).

Even though this thesis provides a solid basis for the experimental exploration of superatomic materials, there are still a few things left to be explored in future studies of these materials. It has already been mentioned that the DFT calculation of cluster based materials revealing even larger clusters is computationally expensive and therefore in many cases inaccessible. This problem can be bypassed by switching to classical models while using the knowledge obtained from DFT calculations, e.g. creating force fields based on the DFT energy calculations of the dimers as a function of the distance. This would give further insight into the possible structures, and their properties, achievable by cluster assemblies. 


\section{Bibliography}

[1] M. C. Chabrie, Compt. Rend. 1907, 144.

[2] J. Demuynck, M.-M. Rohmer, A. Strich, A. Veillard, J. Chem. Phys. 1981, 75, $3443-3453$.

[3] H. W. Kroto, J. R. Heath, S. C. O’Brian, R. E. Curl, R. F.and Smalley, Nature 1985, 318, 162-163.

[4] W. Eberhardt, Surf. Sci. 2002, 500, 242-270.

[5] G. A. Breaux, R. C. Benirschke, T. Sugai, K. B. S., M. F. Jarrold, Phys. Rev. Lett. 2003, 91, 215508.

[6] G. A. Breaux, D. A. Hillman, C. M. Neal, R. C. Benirschke, M. F. Jarrold, J. Am. Chem. Soc. 2004, 126, 8628-8629.

[7] K. J. Taylor, C. Jin, J. Conceicao, L.-S. Wang, O. Cheshnovsky, B. R. Johnson, P. J. Nordlander, R. E. Smalley, J. Chem. Phys. 1990, 93, 7515-7518.

[8] K. G. Steenbergen, N. Gaston, Phys. Chem. Chem. Phys 2013, 15, 15325-15332.

[9] K. G. Steenbergen, N. Gaston, Nano Lett. 2016, 16, 21-26.

[10] A. Sanchez, S. Abbet, U. Heiz, W.-D. Schneider, H. Hkkinen, R. N. Barnett, U. Landman, J. Phys. Chem. A 1999, 48, 9573-9578.

[11] M.-C. Daniel, D. Astruc, Chem. Rev. 2004, 104, 293-346.

[12] S. Yamazoe, K. Koyasu, T. Tsukuda, Acc. Chem. Res. 2014, 47, 816-824. 
[13] J. Oliver-Meseguer, J. R. Cabrero-Antonino, I. Domnguez, A. Leyva-Prez, A. Corma, Science 2012, 338, 1452-1455.

[14] S. N. Khanna, A. W. Castleman, Quantum Phenomena in Clusters and Nanostructures, Springer: New York, 2003.

[15] P. Jena, A. W. Castleman, Proc. Natl. Acad. Sci. U. S. A 2006, 103, 10560-10569.

[16] P. Buffat, J. P. Borel, Phys. Rev. A 1976, 13, 2287-2298.

[17] D. E. Bergeron, A. Castleman Jr., T. Morisato, S. N. Khanna, Science 2004, 304, $84-87$.

[18] D. E. Bergeron, P. J. Roach, A. Castleman Jr., N. O. Jones, S. N. Khanna, Science 2005, 30\%, 231-235.

[19] W. D. Knight, K. Clemenger, W. A. de Heer, W. A. Saunders, M. Y. Chou, M. L. Cohen, Phys. Rev. Lett. 1984, 53, 510.

[20] K. Clemenger, Phys. Rev. B: Condens. Matter Mater. Phys. 1985, 32, 1359-1362.

[21] M. G. Mayer, J. H. D. Jensen, Elementary theory of nuclear shell structure, Wiley: New York, 1955.

[22] M. G. Mayer, Phys. Rev. 1950, 78, 16-21.

[23] M. G. Mayer, Physical Review 1949, 1969-1970.

[24] W. Ekardt, Phy. Rev. B 1984, 29, 1558-1564.

[25] W. Ekardt, Phys. Rev. Lett. 1984, 52, 1925-1928.

[26] N. D. Lang, W. Kohn, Phys. Rev. B 1970, 1.

[27] N. D. Lang, W. Kohn, Phys. Rev. B 1971, 3, 1215.

[28] N. D. Lang, W. Kohn, Phys. Rev. B 1973, 7, 3541. 
[29] N. W. Ashcroft, N. D. Mermin, Solid state Physics, Harcourt College Publishers, 1976.

[30] M. Brack, Rev. Mod. Phys. 1993, 65, 677.

[31] W. A. de Heer, Rev. Mod. Phys. 1993, 65, 611.

[32] J. L. Martins, R. Car, J. Buttet, Surf. Sci. 1981, 106, 265.

[33] A. W. Castleman, S. N. Khanna, J. Phys. Chem. C 2009, 113, 2664-2675.

[34] R. E. Leuchtner, A. C. Harms, A. W. Castleman, J. Chem. Phys. 1989, 91, 275.

[35] B. Leskiw, A. W. Castleman, Chem. Phys. Lett. 2000, 316, 31-36.

[36] A. C. Harms, R. E. Leuchtner, S. W. Sigsworth, A. W. Castleman Jr., J. Am. Chem. Soc. 1990, 112, 5673.

[37] S. N. Khanna, P. Jena, Chem. Phys. Lett. 1994, 219, 479.

[38] X. Li, H. Wu, X.-B. Wang, L.-S. Wang, Phys. Rev. Lett. 1998, 81, 1910-1912.

[39] P. Koirala, M. Willis, B. Kiran, A. K. Kandalam, P. Jena, J. Phys. Chem. C 2010, 114, 16018-16024.

[40] G. L. Gutsev, S. N. Khanna, B. K. Rao, J. P., Phy. Rev. A 1999, 59, 3681-3684.

[41] J. Tong, Y. Li, D. Wu, Z.-R. Li, X.-R. Huang, J. Phys. Chem. A 2011, 115, $2041-2046$.

[42] V. M. Medel, J. U. Reveles, S. N. Khanna, V. Chauhan, P. Sen, A. W. Castleman, Proc Natl Acad Sci U S A. 2011, 108, 10062-10066.

[43] V. Kumar, A. K. Singh, Y. Kawazoe, Nano Lett. 2004, 4, 677-681.

[44] K. Koyasu, J. Akutsu, M.and Atobe, M. Mitsui, A. Nakajima, Chem. Phys. Lett. 2006, 421, 534-539.

[45] H. Hkkinen, Chem. Soc. Rev 2008, 37, 1847-1859. 
[46] A. Desireddy, B. E. Conn, J. Guo, B. Yoon, R. N. Barnett, B. M. Monahan, K. Kirschbaum, W. P. Griffith, R. L. Whetten, U. Landman, T. P. Bigioni, Nature 2013, 501, 399-402.

[47] K. M. Harkness, Y. Tang, A. Dass, J. Pan, N. Kothalawala, V. J. Reddy, D. E. Cliffel, B. Demeler, F. Stellacci, O. M. Bakr, J. A. McLean, Nanoscale 2012, 4, 4269-4274.

[48] M. B. Abreu, C. Powell, A. C. Reber, S. N. Khanna, J. Am. Chem. Soc. 2012, 134, 20507-20512.

[49] D. Schebarchov, N. Gaston, Phys. Chem. Chem. Phys 2011, 13, 21109-21115.

[50] O. Lopez-Acevedo, P. A. Clayborne, H. Hkkinen, Phys. Rev. B 2011, 84, 035434.

[51] K. P. Hall, B. R. C. Theoblad, D. I. Gilmour, D. M. P. Mingos, A. J. Welch, J. Chem. Soc., Chem. Commun. 1982, 528-530.

[52] D. M. P. Mingos, Phil. Trans. R. Soc. Lond. A 1982, 308, 75-83.

[53] B. K. Teo, X. Shi, H. Zhang, J. Am. Chem. Soc. 1992, 115, 2743-2745.

[54] G. Schmid, R. Pfeil, R. Boese, F. Bandermann, S. Meyer, G. H. M. Calis, J. W. A. van der Velden 1981, 114, 3634-3642.

[55] C. E. Briant, B. R. C. Theobald, J. W. White, L. K. Bell, D. M. P. Mingos, A. J. Welch, J. Chem. Soc., Chem. Commun. 1981, 201-202.

[56] M. Brust, M. Walker, D. Bethell, D. J. Schiffrin, R. Whyman, J. Chem. Soc., Chem. Commun. 1994, 801-802.

[57] M. Brust, J. Fink, D. Bethell, D. J. Schiffrin, C. Kiely, J. Chem. Soc., Chem. Commun. 1995, 1655-1656.

[58] H. Hkkinen, R. N. Barnett, U. Landman, Phys. Rev. Lett. 1999, 82, 3264.

[59] H. Hkkinen, M. Walter, H. Grnbeck, J. Phys. Chem. B 2006, 110, 9927-9931. 
[60] L. Garzn, C. Rovira, K. Michaelian, M. R. Beltrn, P. Ordejn, J. Junquera, D. Snchez-Portal, E. Artacho, J. M. Soler, Phys. Rev. Lett. 2000, 85, 5250.

[61] P. D. Jadzinsky, G. Calero, C. J. Ackerson, D. A. Bushnell, R. D. Kornberg, Science 2007, 318, 430-433.

[62] M. Walter, J. Akola, O. Lopez-Acevedo, P. D. Jadzinsky, G. Calero, C. J. Ackerson, R. L. Whetten, H. Grnbeck, H. Hkkinen, PNAS 2008, 105, 9157-9162.

[63] H. Qian, W. T. Eckenhoff, Y. Zhu, T. Pintauer, R. Jin, J. Am. Chem. Soc. 2010, 132, 8280-8281.

[64] A. Dass, J. Am. Chem. Soc. 2009, 131, 11666-11667.

[65] N. K. Chaki, Y. Negishi, H. Tsunoyama, Y. Shichibu, T. Tsukuda, J. Am. Chem. Soc. 2008, 130, 8608-8610.

[66] Y. Negishi, C. Sakamoto, T. Ohyama, T. Tsukuda, J. Phys. Chem. Lett. 2012, 3, $1624-1628$.

[67] O. Lopez-Acevedo, H. Tsunoyama, T. Tsukuda, H. Hkkinen, C. M. Aikens, J. Am. Chem. Soc. 2010, 132, 8210-8218.

[68] Y. Pei, Y. Gao, N. Shao, X. C. Zeng, J. Am. Chem. Soc. 2009, 131, 13619-13621.

[69] O. Lopez-Acevedo, J. Akola, R. L. Whetten, H. Grnbeck, H. Hkkinen, J. Phys. Chem. C 2009, 113, 5035-5038.

[70] Y. Pei, Y. Gao, X. C. Zeng, J. Am. Chem. Soc. 2008, 130, 7830-7832.

[71] M. W. Heaven, A. Dass, P. S. White, K. M. Holt, R. W. Murray, J. Am. Chem. Soc. 2008, 130, 3754-3755.

[72] K. A. Kacprzak, L. Lehtovaara, J. Akola, O. Lopez-Acevedo, H. Hkkinen, Phys. Chem. Chem. Phys. 2009, 11, 7123-7129.

[73] M. Zhu, C. M. Aikens, F. J. Hollander, G. C. Schatz, R. Jin, J. Am. Chem. Soc. 2008, 130, 5883-5885. 
[74] T. Stoll, E. Sgr, J. W. Jarrett, J. Rhault, A. Oriana, L. Sala, F. Branchi, G. Cerullo, K. L. Knappenberger, J. Am. Chem. Soc. 2016, 138, 1788-1791.

[75] Z. Wu, M. Wang, J. Yang, X. Zheng, W. Cai, G. Meng, H. Qian, H. Wang, R. Jin, Small 2012, 13, 2028-2035.

[76] Z. Wu, R. Jin, Nano Lett. 2010, 10, 2568-2573.

[77] M. Zhu, C. M. Aikens, M. P. Hendrich, R. Gupta, H. Qian, G. C. Schatz, R. Jin, J. Am. Chem. Soc. 2009, 131, 2490-2492.

[78] Z. Liu, M. Zhu, X. Meng, G. Xu, R. Jin, J. Phys. Chem. Lett. 2011, 2, 2104-2109.

[79] C. A. Fields-Zinna, M. C. Crowe, A. Dass, W. J. E. F., R. W. Murray, Langmuir 2009, 25, 7704-7710.

[80] Y. Negishi, W. Kurashige, Y. Niihori, K. Nobusada, Phys. Chem. Chem. Phys. 2013, 15, 18736-18751.

[81] Y. Negishi, W. Kurashige, Y. Niihori, T. Iwasa, K. Nobusada, Phys. Chem. Chem. Phys. 2010, 12, 6219-6225.

[82] S. Sharma, S. Yamazoe, T. Ono, W. Kurashige, Y. Niihori, K. Nobusada, T. Tsukuda, Y. Negishi, Dalton Trans. 2016, 45, 18064-18068.

[83] Y. Negishi, K. Munakata, W. Ohgake, K. Nobusada, J. Phys. Chem. Lett. 2012, 3, 2209-2214.

[84] Y. Niihori, W. Kurashige, M. Matsuzaki, Y. Negishi, Nanoscale 2013, 5, 508-512.

[85] Y. Negishi, W. Kurashige, Y. Kobayashi, S. Yamazoe, N. Kojima, M. Seto, T. Tsukuda, J. Phys. Chem. Lett. 2013, 4, 3579-3583.

[86] Y. Negishi, T. Iwai, M. Ide, Chem. Commun. 2010, 46, 4713-4715.

[87] H. Qian, D. Jiang, G. Li, C. Gayathri, A. Das, R. R. Gil, R. Jin, J. Am. Chem. Soc. 2012, 134, 16159-16162. 
[88] D. Jiang, S. Dai, Inorg. Chem. 2009, 48, 2720-2722.

[89] J. Schacht, N. Gaston, Chemphyschem. 2016, 17, 3237-3244.

[90] E. B. Guidez, V. Mkinen, H. Hkkinen, C. M. Aikens, J. Phys. Chem. C 2012, $116,20617-20624$.

[91] H. Qian, R. Jin, Nano Lett. 2009, 9, 4083-4087.

[92] M. Schulz-Dobrick, M. Jansen, Eur. J. Inorg. Chem. 2006, 2006, 4498-4502.

[93] M. Schulz-Dobrick, M.and Jansen, Z. Anorg. Allg. Chem. 2007, 633, 2326-2331.

[94] M. Schulz-Dobrick, M. Jansen, Inorg. Chem. 2007, 46, 4380-4382.

[95] M. Schulz-Dobrick, M. Jansen, Angew. Chem. 2008, 47, 2256-2259.

[96] X. Roy, C.-H. Lee, A. C. Crowther, C. L. Schenck, T. Besara, R. A. Lalancette, T. Siegrist, P. W. Stephens, L. E. Brus, P. Kim, M. L. Steigerwald, C. Nuckolls, Science 2013, 341, 157-160.

[97] S. A. Claridge, A. W. Castleman, S. N. Khanna, C. B. Murray, A. Sen, P. S. Weiss, ACS Nano 2009, 3, 244-255.

[98] E. V. Shevchenko, D. V. Talapin, C. B. Murray, S. O’Brien, J. Am. Chem. Soc. 2006, 128, 3620-3637.

[99] T. Yoshida, K. Nomiya, S. Matsunaga, Dalton Trans. 2012, 41, 10085-10090.

[100] W.-H. Fang, L. Zhang, J. Zhang, Chem. Commun. 2017, 53, 3949-3951.

[101] G. Borstel, U. Lammers, A. Mananes, J. A. Alonso, Nuclear Physics Concepts in Atomic Cluster Physics, Springer, Berlin, 1992.

[102] R. Monnier, J. P. Perdew, Phys. Rev. B 1978, 17, 2595-2611.

[103] S. Rdiger, E. Kemnitz, Dalton Trans. 2008, 1117-1127. 
[104] A. Dimitrov, J. Koch, S. I. Troyanov, E. Kemnitz, Eur. J. Inorg. Chem. 2009, 2009, 5299-5301.

[105] S. Wuttke, S. M. Coman, J. Krhnert, F. C. Jentoft, E. Kemnitz, Catal. Today 2010, 152, 2-10.

[106] R. Knig, G. Scholz, M. Veiczi, C. Jger, S. I. Troyanov, E. Kemnitz, Dalton Trans. 2011, 40, 8701-8710

[107] M. Planck, Ann. Phys. 1900, 309, 553.

[108] L. De Broglie, Annales de Physique 1925, 10.

[109] E. Schrdinger, Ann. Phys. 1926, 81, 109.

[110] E. Schrdinger, Ann. Phys. 1926, 79, 489.

[111] E. Schrdinger, Ann. Phys. 1926, 80, 437.

[112] E. Schrdinger, Ann. Phys. 1926, 69, 361-376.

[113] M. Born, Z. Physik 1926, 37, 863-867.

[114] M. Born, Z. Physik 1927, 40, 167-192.

[115] M. Born, R. Oppenheimer, Ann. Phys. 1927, 389, 457-484.

[116] D. R. Hartree, Proc. Camb. Phil. Soc. 1928, 24, 89.

[117] J. C. Slater, Phys. Rev. 1951, 81, 385.

[118] A. Szabo, N. S. Ostlund, Modern Quantum Chemistry, 1 Aufl., Dover Publication, Inc: Mineola,New York, 1996.

[119] C. C. J. Roothaan, Rev. Mod. Phys. 1951, 23, 69-89.

[120] G. G. Hall, Proc. R. Soc. London Ser. A 1951, 205, 541-552.

[121] W. Koch, M. C. Holthausen, A Chemist's Guide to Density Functional Theory, Wiley-VCH Verlag GmbH, 2001. 
[122] L. H. Thomas, Math. Proc. Cambridge Phil. Soc. 1927, 23, 542-548.

[123] E. Fermi, Rend. Accad. Naz. Lincei 1927, 6, 602-607.

[124] P. Hohenberg, W. Kohn, Phys. Rev. 1964, 136, B864-B871.

[125] W. Kohn, L. J. Sham, Phys. Rev. 1965, 140, A1133-A1138.

[126] P. A. M. Dirac, Math. Proc. Cambridge Phil. Soc. 1930, 26, 376-385.

[127] S. H. Vosko, L. Wilk, M. Nusair, Can. J. Phys. 1980, 58, 1200-1211.

[128] J. P. Perdew, Y. Wang, Phys. Rev. B 1992, 45, 13244-13249.

[129] J. P. Perdew, K. Burke, M. Ernzerhof, Phys. Rev. Lett. 1996, 77, 3865-3868.

[130] J. P. Perdew, K. Burke, M. Ernzerhof, Phys. Rev. Lett. 1997, 78, 1396-1396.

[131] A. D. Becke, J. Chem. Phys 1993, 98, 5648-5652.

[132] C. Lee, W. Wang, R. G. Parr, Phys. Rev. B 1988, 37, 785-789.

[133] S. Grimme, J. Antony, S. Ehrlich, H. Krieg, J. Chem. Phys. 2010, 132, 154104.

[134] S. Grimme, S. Ehrlich, L. Goerigk, J. Comp. Chem. 2011, 32, 1456-1465.

[135] F. Jensen, Introduction to Computational Chemistry, Jhon Wiley Sons, Ltd, 2007.

[136] H. Hellmann, J. Chem. Phys. 1935, 3, 61.

[137] A. E. Reed, R. B. Weinstock, F. Weinhold, J. Chem. Phys. 1985, 83, 735-746.

[138] R. S. Mulliken, J. Chem. Phys. 1955, 23, 1833-1840.

[139] R. S. Mulliken, J. Chem. Phys. 1955, 23, 1841-1846.

[140] G. Henkelman, A. Arnaldsson, H. Jonsson, Compt. Mater. Sci. 2006, 36, 354-360.

[141] E. Sanville, S. D. Kenny, R. Smith, G. Henkelman, J. Comput. Chem. 2007, 28, 899-908. 
[142] W. Tang, E. Sanville, G. Henkelman, J. Phys.: Condens. Matter 2009, 21, 084204.

[143] H. Jonsson, Phys. Rev. Lett. 1994, 72, 1124-1127.

[144] G. Henkelman, H. Jonsson, J. Chem. Phys 2000, 113, 9978-9985.

[145] T. Ziegler, A. Rauk, Theoretica chimica acta 1977, 46, 1-10.

[146] R. J. Singh, Solid State Physics, Pearson Education India, 2011.

[147] K. Kopitzki, P. Herzog, Einführung in die Festkörperphysik, Teubner Verlag, 2001.

[148] F. Bloch, Z. Phys. 1929, 52, 555-600.

[149] R. Dronskowski, Computational Chemistry of Solid State Materials, Wiley-VCH, Weinheim, 2005.

[150] E. Wigner, F. Seitz, Phys. Rev. 1933, 43, 804-810.

[151] H. J. Monkhorst, J. D. Pack, Phys. Rev. B 1976, 146, 5188.

[152] M. Defranceschi, C. Le Bris, Mathematical Models and Methods for Ab Initio Quantum Chemistry, Springer, 2000.

[153] G. B. Bachelet, D. R. Hamann, M. Schlüter, Phys. Rev. B 1982, 26, 4199-4228.

[154] D. Vanderbilt, Phys. Rev. B 1990, 41, 7892-7895.

[155] P. E. Blöchl, Phys. Rev. B 1994, 50, 17953-17979.

[156] G. Kresse, D. Joubert, Phys. Rev. B 1999, 59, 1758-1775.

[157] D. Schebarchov, N. Gaston, Phys. Chem. Chem. Phys. 2012, 14, 9912-9922.

[158] O. Lopez-Acevedo, R. L. Akola, J.and Whetten, H. Grnbeck, H. Hkkinen, J. Phys. Chem. C 2009, 113, 5035-5038.

[159] G. Kresse, J. Hafner, Phys. Rev. B 1993, 47, 558.

[160] G. Kresse, J. Hafner, Phys. Rev. B 1994, 49, 14251. 
[161] G. Kresse, J. Hafner, J. Phys.: Condm. Matter 1994, 6, 8245.

[162] G. Kresse, J. Furthmüller, Comput. Mat. Sci. 1996, 6, 15-50.

[163] G. Kresse, J. Furthmüller, Phys. Rev. B 1996, 54, 11169.

[164] TURBOMOLE V7.0 2015, a development of University of Karlsruhe and Forschungszentrum Karlsruhe GmbH, 1989-2007, TURBOMOLE GmbH, since 2007; available from http://www . turbomole.com.

[165] G. te Velde, F. M. Bickelhaupt, E. J. Baerends, C. Fonseca Guerra, S. J. A. van Gisbergen, J. G. Snijders, T. Ziegler, J. Comput. Chem. 2001, 22, 931-967.

[166] C. Fonseca Guerra, J. G. Snijders, G. te Velde, E. J. Baerends, Theoretical Chemistry Accounts 1998, 99, 391.

[167] Y. Negishi, K. Igarashi, K. Munakata, W. Ohgake, K. Nobusada, Chem. Commun. 2012, 48, 660-662.

[168] D. Jiang, R. L. Whetten, Phys. Rev. B 2009, 80, 115402.

[169] M. A. Tofanelli, K. Salorinne, T. W. Ni, S. Malola, B. Newell, B. Phillips, H. Hkkinen, C. J. Ackerson, Chem. Sci. 2016, 7, 1882-1890.

[170] W. M. Haynes, CRC Handbook of Chemistry 8 Physics, Vol. 95, Taylor \& Francis, New York, 2014.

[171] B. Fricke, J. Chem. Phys 1986, 84, 862.

[172] I. K. Dmitrieva, G. I. Plindov, Phys. Scr. 1983, 27, 402-406.

[173] L. Hammerschmidt, J. Schacht, N. Gaston, Phys. Chem. Chem. Phys. 2016, 18, $32541-32550$.

[174] J. P. Perdew, A. Zunger, Phys. Rev. B: Condens. Matter Mater. Phys. 1981, 23, 5048. 
[175] J. P. Perdew, A. Ruzsinszky, G. I. Csonka, O. A. Vydrov, G. E. Scuseria, L. A. Constantin, X. Zhou, K. Burke, Phys. Rev. Lett. 2008, 100, 136406.

[176] A. Tkatchenko, M. Scheffler, Phys. Rev. Lett 2009, 102, 073005.

[177] M. Swart, Chem. Phys. Lett. 2013, 580, 166-171.

[178] E. Van Lenthe, E. J. Baerends, J. Comp. Chem. 2003, 24, 1142-1156.

[179] E. van Lenthe, E. J. Baerends, J. G. Snijders, J. Chem. Phys. 1993, 99, 4597.

[180] E. van Lenthe, E. J. Baerends, J. G. Snijders, J. Chem. Phys. 1994, 101, 9783.

[181] E. van Lenthe, A. Ehlers, E. J. Baerends, J. Chem. Phys. 1999, 110, 8943.

[182] G. K. H. Madsen, D. J. Singh, Comput. Phys. Commun. 2006, $175,67$.

[183] A. D. Becke, K. E. Edgecombe, J. Chem. Phys. 1990, 92, 5397.

[184] A. Lebon, A. Aguado, A. Vega, Phys. Chem. Chem. Phys. 2015, 17, 28033-28043.

[185] A. Aguado, A. Vega, A. Lebon, B. von Issendorff, Angew. Chem. 2015, 127, 21392143.

[186] D. Mollenhauer, N. Gaston, Phys. Chem. Chem. Phys. 2016, 18, 29686-29697.

[187] J. Zhao, M. Feng, J. Yang, H. Petek, ACS Nano 2009, 3, 853-864.

[188] L. Pauling, J. Am. Chem. Soc. 1929, 51, 1010-1026.

[189] E. Pahl, D. Figgen, A. Borschevsky, K. A. Peterson, P. Schwerdtfeger, Theor. Chem. Acc. 2011, 129, 651-656.

[190] R. D. van Zee, S. C. Blankespoor, T. S. Zwier, J. Chem. Phys. 1988, 88, 46504654 .

[191] M. Shibuta, T. Ohta, M. Nakaya, H. Tsunoyama, T. Eguchi, A. Nakajima, J. Am. Chem. Soc. 2015, 137, 14015-14018.

[192] S. Malola, H. Hkkinen, J. Phys. Chem. Lett. 2011, 2, 2316-2321. 
[193] W. Kurashige, Y. Negishi, Journal of Cluster Science 2012, 23, 365-374.

[194] W. Kurashige, M. Yamaguchi, K. Nobusada, Y. Negishi, J. Phys. Chem. Lett. 2012, 3, 2649-2652.

[195] J. Jung, S. Kang, Y.-K. Han, Nanoscale 2012, 4, 4206-4210.

[196] P. Avouris, Nano Lett. 2010, 10, 4285-4294.

[197] K. A. Ritter, J. W. Lyding, Nature Materials 2009, 8, 235-242.

[198] A. H. Castro Neto, F. Guinea, N. M. R. Peres, K. S. Novoselov, A. K. Geim, Rev. Mod. Phys. 2009, 81, 109.

[199] H. Hamada, Catal. Today 1994, 22, 21-40.

[200] J. Tao, J. P. Perdew, V. N. Staroverov, G. E. Scuseria, Phys. Rev. Lett. 2003, 91, 146401.

[201] A. Klamt, J. Phys. Chem. 1995, 99, 2224-2235.

[202] A. Klamt, V. Jonas, T. Brger, J. C. Lohrenz, J. Phys. Chem. 1998, 102, 50745085 .

[203] J. P. Perdew, Phys. Rev. B 1986, 33, 8822-8824.

[204] A. D. Becke, Phys. Rev. A 1988, 38, 3098-3100.

[205] H. Eyring, Chem. Rev. 1935, 17, 65-77.

[206] From personal communication with Johannes Horst Budau. 